Cochrane Database of Systematic Reviews

\title{
Hospitalisation in short-stay units for adults with internal medicine diseases and conditions (Review)
}

Strøm C, Stefansson JS, Fabritius ML, Rasmussen LS, Schmidt TA, Jakobsen JC

Strøm C, Stefansson JS, Fabritius ML, Rasmussen LS, Schmidt TA, Jakobsen JC.

Hospitalisation in short-stay units for adults with internal medicine diseases and conditions.

Cochrane Database of Systematic Reviews 2018, Issue 8. Art. No.: CD012370.

DOI: 10.1002/14651858.CD012370.pub2.

www.cochranelibrary.com 
TABLE OF CONTENTS

HEADER

ABSTRACT

PLAIN LANGUAGE SUMMARY

SUMMARY OF FINDINGS

BACKGROUND

OBJECTIVES

METHODS

Figure 1.

Figure 2.

Figure 3.

RESULTS

DISCUSSION

AUTHORS' CONCLUSIONS

ACKNOWLEDGEMENTS

REFERENCES

CHARACTERISTICS OF STUDIES

DATA AND ANALYSES

Analysis 1.1. Comparison 1 Mortality in participants treated in short-stay unit vs usual care, Outcome 1 Mortality at time point closest to 90 days.

Analysis 1.2. Comparison 1 Mortality in participants treated in short-stay unit vs usual care, Outcome 2 Mortality at time point closest to 90 days best-worst case scenario.

Analysis 1.3. Comparison 1 Mortality in participants treated in short-stay unit vs usual care, Outcome 3 Mortality at time point closest to 90 days worst-best case scenario.

Analysis 1.4. Comparison 1 Mortality in participants treated in short-stay unit vs usual care, Outcome 4 Mortality at time point closest to 90 days published trials.

Analysis 1.5. Comparison 1 Mortality in participants treated in short-stay unit vs usual care, Outcome 5 Mortality at time point closest to 90 days and outcome assessed within 6 months of randomisation.

Analysis 1.6. Comparison 1 Mortality in participants treated in short-stay unit vs usual care, Outcome 6 Mortality at time point closest to 90 days multipurpose unit vs specialised unit.

Analysis 1.7. Comparison 1 Mortality in participants treated in short-stay unit vs usual care, Outcome 7 Mortality at time point closest to 90 days non-protocol-based vs protocol-based care.

Analysis 1.8. Comparison 1 Mortality in participants treated in short-stay unit vs usual care, Outcome 8 Mortality at time point closest to 90 days older participants vs younger participants.

Analysis 1.9. Comparison 1 Mortality in participants treated in short-stay unit vs usual care, Outcome 9 Mortality at maximum follow-up.

Analysis 1.10. Comparison 1 Mortality in participants treated in short-stay unit vs usual care, Outcome 10 Mortality at maximum follow-up best-worst case scenario.

Analysis 1.11. Comparison 1 Mortality in participants treated in short-stay unit vs usual care, Outcome 11 Mortality at maximum follow-up worst-best case scenario.

Analysis 1.12. Comparison 1 Mortality in participants treated in short-stay unit vs usual care, Outcome 12 Mortality at maximum follow-up published trials.

Analysis 1.13. Comparison 1 Mortality in participants treated in short-stay unit vs usual care, Outcome 13 Mortality at maximum follow-up and outcome assessed within 6 months of randomisation.

Analysis 1.14. Comparison 1 Mortality in participants treated in short-stay unit vs usual care, Outcome 14 Mortality at maximum follow-up multipurpose unit vs specialised unit.

Analysis 1.15. Comparison 1 Mortality in participants treated in short-stay unit vs usual care, Outcome 15 Mortality at maximum follow-up non-protocol-based vs protocol-based care.

Analysis 1.16. Comparison 1 Mortality in participants treated in short-stay unit vs usual care, Outcome 16 Mortality at maximum follow-up older vs younger participants.

Analysis 2.1. Comparison 2 Serious adverse events in participants treated in short-stay unit vs usual care, Outcome 1 Serious adverse events at time point closest to 90-days.

Analysis 2.2. Comparison 2 Serious adverse events in participants treated in short-stay unit vs usual care, Outcome 2 Serious adverse events at time point closest to 90 days best-worst case scenario. 
Analysis 2.3. Comparison 2 Serious adverse events in participants treated in short-stay unit vs usual care, Outcome 3 Serious adverse events at time point closest to 90 days worst-best case scenario.

Analysis 2.4. Comparison 2 Serious adverse events in participants treated in short-stay unit vs usual care, Outcome 4 Serious adverse events at time point closest to 90-days published trials.

Analysis 2.5. Comparison 2 Serious adverse events in participants treated in short-stay unit vs usual care, Outcome 5 Serious adverse events at time point closest to 90-days and outcome assessed within 6 months of randomisation.

Analysis 2.6. Comparison 2 Serious adverse events in participants treated in short-stay unit vs usual care, Outcome 6 Serious adverse events at time point closest to 90 days multipurpose unit vs specialised unit.

Analysis 2.7. Comparison 2 Serious adverse events in participants treated in short-stay unit vs usual care, Outcome 7 Serious adverse events at time point closest to 90-days non-protocol-based vs protocol-based care.

Analysis 2.8. Comparison 2 Serious adverse events in participants treated in short-stay unit vs usual care, Outcome 8 Serious adverse events at time point closest to 90-days older vs younger participants.

Analysis 2.9. Comparison 2 Serious adverse events in participants treated in short-stay unit vs usual care, Outcome 9 Serious adverse events at maximum follow-up.

Analysis 2.10. Comparison 2 Serious adverse events in participants treated in short-stay unit vs usual care, Outcome 10 Serious adverse events multipurpose unit at maximum follow-up best-worst case scenario.

Analysis 2.11. Comparison 2 Serious adverse events in participants treated in short-stay unit vs usual care, Outcome 11 Serious adverse events multipurpose unit at maximum follow-up worst-best case scenario.

Analysis 2.12. Comparison 2 Serious adverse events in participants treated in short-stay unit vs usual care, Outcome 12 Serious adverse events multipurpose unit at maximum follow-up published trials.

Analysis 2.13. Comparison 2 Serious adverse events in participants treated in short-stay unit vs usual care, Outcome 13 Serious adverse events multipurpose unit at maximum follow-up and outcome assessed within 6 months of randomisation. ............. Analysis 2.14. Comparison 2 Serious adverse events in participants treated in short-stay unit vs usual care, Outcome 14 Serious adverse events at maximum follow-up multipurpose unit vs specialised unit.

Analysis 2.15. Comparison 2 Serious adverse events in participants treated in short-stay unit vs usual care, Outcome 15 Serious adverse events at maximum follow-up non-protocol-based vs protocol-based care.

Analysis 2.16. Comparison 2 Serious adverse events in participants treated in short-stay unit vs usual care, Outcome 16 Serious adverse events at maximum follow-up older participants vs younger participants.

Analysis 2.17. Comparison 2 Serious adverse events in participants treated in short-stay unit vs usual care, Outcome 17 Serious adverse events at time point closest to 90 days.

Analysis 2.18. Comparison 2 Serious adverse events in participants treated in short-stay unit vs usual care, Outcome 18 Serious adverse events at time point closest to 90 days w/o Farkouh 1998.

Analysis 3.1. Comparison 3 Hospital readmissions in participants treated in short-stay unit vs usual care, Outcome 1 Hospital readmissions at the time point closest to 90 days.

Analysis 3.2. Comparison 3 Hospital readmissions in participants treated in short-stay unit vs usual care, Outcome 2 Hospital readmissions at the time point closest to 90 days best-worst case scenario.

Analysis 3.3. Comparison 3 Hospital readmissions in participants treated in short-stay unit vs usual care, Outcome 3 Hospital readmissions at the time point closest to 90 days worst-best case scenario.

Analysis 3.4. Comparison 3 Hospital readmissions in participants treated in short-stay unit vs usual care, Outcome 4 Hospital readmissions at the time point closest to 90 days published trials.

Analysis 3.5. Comparison 3 Hospital readmissions in participants treated in short-stay unit vs usual care, Outcome 5 Hospital readmissions at the time point closest to 90 days outcome assessed within 6 months of randomisation.

Analysis 3.6. Comparison 3 Hospital readmissions in participants treated in short-stay unit vs usual care, Outcome 6 Hospital readmissions at the time point closest to 90 days multipurpose units vs specialised units.

Analysis 3.7. Comparison 3 Hospital readmissions in participants treated in short-stay unit vs usual care, Outcome 7 Hospital readmissions at the time point closest to 90 days non-protocol-based vs protocol-based care.

Analysis 3.8. Comparison 3 Hospital readmissions in participants treated in short-stay unit vs usual care, Outcome 8 Hospital readmissions at the time point closest to 90 days older vs younger participants.

Analysis 3.9. Comparison 3 Hospital readmissions in participants treated in short-stay unit vs usual care, Outcome 9 Hospital readmissions at the time point maximum follow-up.

Analysis 3.10. Comparison 3 Hospital readmissions in participants treated in short-stay unit vs usual care, Outcome $10 \mathrm{Hospital}$ readmissions at the time point maximum follow-up best-worst case scenario.

Analysis 3.11. Comparison 3 Hospital readmissions in participants treated in short-stay unit vs usual care, Outcome $11 \mathrm{Hospital}$ readmissions at the time point maximum follow-up worst-best case scenario.

Analysis 3.12. Comparison 3 Hospital readmissions in participants treated in short-stay unit vs usual care, Outcome $12 \mathrm{Hospital}$ readmissions at the time point maximum follow-up published trials. 
Analysis 3.13. Comparison 3 Hospital readmissions in participants treated in short-stay unit vs usual care, Outcome 13 Hospital readmissions at the time point maximum follow-up outcome assessed within 6 months of randomisation.

Analysis 3.14. Comparison 3 Hospital readmissions in participants treated in short-stay unit vs usual care, Outcome $14 \mathrm{Hospital}$ readmissions at the time point maximum follow-up multipurpose units vs specialised units.

Analysis 3.15. Comparison 3 Hospital readmissions in participants treated in short-stay unit vs usual care, Outcome $15 \mathrm{Hospital}$ readmissions at the time point maximum follow-up non-protocol-based vs protocol-based care.

Analysis 3.16. Comparison 3 Hospital readmissions in participants treated in short-stay unit vs usual care, Outcome $16 \mathrm{Hospital}$ readmissions at the time point maximum follow-up older vs younger participants.

Analysis 3.17. Comparison 3 Hospital readmissions in participants treated in short-stay unit vs usual care, Outcome $17 \mathrm{Hospital}$ readmissions at the time point closest to 90 days w/o Strøm 2017a.

Analysis 3.18. Comparison 3 Hospital readmissions in participants treated in short-stay unit vs usual care, Outcome $18 \mathrm{Hospital}$ readmissions at the time point maximum follow-up w/o Strøm 2017 a.

Analysis 3.19. Comparison 3 Hospital readmissions in participants treated in short-stay unit vs usual care, Outcome 19 Hospital readmissions at the time point maximum follow-up w/o Strøm 2017a and Miller 2010.

ADDITIONAL TABLES

APPENDICES

CONTRIBUTIONS OF AUTHORS

DECLARATIONS OF INTEREST

SOURCES OF SUPPORT

DIFFERENCES BETWEEN PROTOCOL AND REVIEW

INDEX TERMS 
[Intervention Review]

\section{Hospitalisation in short-stay units for adults with internal medicine diseases and conditions}

Camilla Strøm¹, Jakob S Stefansson², Maria Louise Fabritius², Lars S Rasmussen², Thomas A Schmidt¹, Janus C Jakobsen ${ }^{3,4}$

1Department of Emergency Medicine, Holbaek Hospital, University of Copenhagen, Holbaek, Denmark. 2Department of Anaesthesia, Centre of Head and Orthopaedics, Rigshospitalet, University of Copenhagen, Copenhagen, Denmark. ${ }^{3}$ Cochrane Hepato-Biliary Group, Copenhagen Trial Unit, Centre for Clinical Intervention Research, Department 7812, Rigshospitalet, Copenhagen University Hospital, Copenhagen, Denmark. ${ }^{4}$ Department of Cardiology, Holbaek Hospital, Holbaek, Denmark

Contact address: Camilla Strøm, Department of Emergency Medicine, Holbaek Hospital, University of Copenhagen, Holbaek, 4300 , Denmark.cstr@regionsjaelland.dk, camilla007@gmail.com.

Editorial group: Cochrane Effective Practice and Organisation of Care Group.

Publication status and date: New, published in Issue 8, 2018.

Citation: Strøm C, Stefansson JS, Fabritius ML, Rasmussen LS, Schmidt TA, Jakobsen JC. Hospitalisation in short-stay units for adults with internal medicine diseases and conditions. Cochrane Database of Systematic Reviews 2018, Issue 8. Art. No.: CD012370. DOI: 10.1002/14651858.CD012370.pub2.

Copyright () 2018 The Cochrane Collaboration. Published by John Wiley \& Sons, Ltd.

\section{A B S T R A C T}

\section{Background}

Short-stay units are hospital units that provide short-term care for selected patients. Studies have indicated that short-stay units might reduce admission rates, time of hospital stays, hospital readmissions and expenditure without compromising the quality of care. Shortstay units are often defined by a target patient category, a target function, and a target time frame. Hypothetically, short-stay units could be established as part of any department, but this review focuses on short-stay units that provide care for participants with internal medicine diseases and conditions.

\section{Objectives}

To assess beneficial and harmful effects of short-stay unit hospitalisation compared with usual care in people with internal medicine diseases and conditions.

\section{Search methods}

We searched CENTRAL, MEDLINE, Embase, three other databases and two trials registers up to 13 December 2017 together with reference checking, citation searching and contact with study authors to identify additional studies. We also searched several grey literature sources and performed a forward citation search for included studies.

\section{Selection criteria}

We included randomised trials and cluster-randomised trials, comparing hospitalisation in a short-stay unit with usual care (hospitalisation in a traditional hospital ward or other services). We defined a short-stay unit to be a hospital ward where the targeted length of stay in hospital for patients was five days or less. We included both multipurpose and specialised short-stay units. Participants were adults admitted to hospital with an internal medicine disease or condition. We excluded surgical, obstetric, psychiatric, gynaecological, and ambulatory participants. Trials were included irrespective of publication status, date, and language.

\section{Data collection and analysis}

We used standard methodological procedures expected by Cochrane. Two review authors independently extracted data and assessed the risk of bias of each included trial. We measured intervention effect sizes by meta-analyses for two primary outcomes, mortality and serious adverse events, and one secondary outcome, hospital readmission. We narratively reported the following important outcomes: quality of 
life, activities of daily living, non-serious adverse events, and costs. We used risk ratio differences of $15 \%$ for mortality and of $20 \%$ for serious adverse events for minimal relevant clinical consideration. We rated the certainty of the evidence and the strength of recommendations of the outcomes using the GRADE approach.

\section{Main results}

We included 19 records reporting on 14 randomised trials with a total of 2872 participants. One trial was ongoing. Thirteen trials evaluated short-stay unit hospitalisation for six specific conditions (acute decompensated heart failure (one trial), asthma (one trial), atrial fibrillation (one trial), chest pain (seven trials), syncope (two trials), and transient ischaemic attack (one trial)) and one trial investigated participants presenting with miscellaneous internal medicine disease and conditions. The components of the intervention differed among the trials as dictated by the trial participants' condition. All included trials were at high risk of bias.

The certainty of the evidence for all outcomes was very low. Consequently, it is uncertain whether hospitalisation in short-stay units compared with usual care reduces mortality (risk ratio (RR) $0.73,95 \%$ confidence interval (CI) 0.47 to 1.15 ) 5 trials (seven additional trials reporting on 1299 participants reported no deaths in either group)); serious adverse events (RR 0.95, 95\% $\mathrm{Cl} 0.59$ to $1.54 ; 7$ trials (one additional trial with 108 participants reported no serious adverse events in either group)), and hospital readmission ( $\mathrm{RR} 0.80,95 \% \mathrm{Cl} 0.54$ to $1.19,8$ trials (one additional trial with 424 participants did not report results for participants)). There was not enough information to confirm or refute that short-stay unit hospitalisation had relevant effects on quality of life, activities of daily living, non-serious adverse events, and costs.

\section{Authors' conclusions}

Overall, the quantity and the certainty of the evidence was very low. Consequently, it is uncertain whether there are any beneficial or harmful effects of short-stay unit hospitalisation for adults with internal medicine diseases and conditions - more trials comparing the effects of short-stay units with usual care are needed. Such trials ought to be conducted with low risk of bias and low risks of random errors to improve the overall confidence in the evidence.

\section{PLAIN LANGUAGE SUMMARY}

\section{Hospitalisation in short-stay units for adults with internal medicine diseases and conditions}

\section{What is the aim of this review?}

To find out whether short stays in hospital in short-stay units improve outcomes in adults with internal medicine diseases and conditions compared to usual care.

\section{Key messages}

We are unsure about the effect of short-stay unit hospitalisation for adults with internal medicine diseases and conditions compared to usual care. The evidence was uncertain for several important reasons; including not having enough data, differences among participants and co-interventions, and problems with the methods that the trials used that could have led to false results. We need more high-quality trials to test the impact of short-stay unit hospitalisation on individual patients and costs.

\section{What was studied in the review?}

Short-stay units are hospital units that provide short-term care in selected patients. Their services are often defined by the type of patient, the unit's function, and a time frame. Studies have indicated that short-stay units may reduce the number of people admitted to hospital, the length of time they spend in hospital, the number of people who have to go back into hospital (readmission), and costs, without losing any quality of care, but a thorough evaluation of effects of short-stay unit hospitalisation compared with usual care (mainly hospitalisation in a traditional hospital ward) was lacking before we conducted the present review. The review focused on short-stay unit hospitalisation for internal medicine diseases and conditions, such as pneumonia or chest pain. We compared the effect of short-stay unit hospitalisation with usual care by looking at deaths (mortality), serious problems (serious adverse events), quality of life, activities of daily living (such as managing housework or medications ), hospital readmission, non-serious adverse events, and costs.

\section{What are the main results of the review?}

The review authors found 14 relevant trials with a total of 2872 participants. All trials were randomised trials, i.e. people participating in the trials had been assigned by chance alone to either hospitalisation in a short-stay unit or a control group that received usual care. Randomised trials are considered to be the most reliable trial design to test effects of an intervention.

Thirteen trials evaluated short-stay unit hospitalisation for six specific conditions (asthma, atrial fibrillation (irregular heartbeat), chest pain, decompensated (worsening of the signs of) heart failure, syncope (losing consciousness due to a fall in blood pressure), and transient ischaemic attack (mini stroke)) and one trial did not specify which condition its participants had. We identified one ongoing trial. The components of short-stay unit hospitalisation differed among the trials depending on the trial participants' conditions. All of the included trials had problems with their methods that potentially could have led to false results. We were uncertain whether there was any difference 
between short-stay unit hospitalisation and usual care for reducing mortality, serious adverse events, and hospital readmissions. We were not able to combine and examine results for any other outcomes, because the trials used different ways of measuring (e.g. using different scales), or did not give enough data, or reported their results in a way that meant we could not use them. Individual trials said that short-stay unit hospitalisation led to higher quality of life, fewer non-serious adverse events, and lower costs. However, we cannot be certain about this evidence and need to be careful about interpreting the trials' results; all included trials were at high risk of errors, which questions the validity of these results and we cannot exclude that the findings were merely due to the play of chance. It is crucial to validate the findings in larger, well-conducted trials.

\section{How up-to-date is this review?}

The review authors searched for trials that had been published before 13 December 2017. 


\begin{tabular}{|c|c|c|c|c|c|c|c|}
\hline \multirow{11}{*}{ 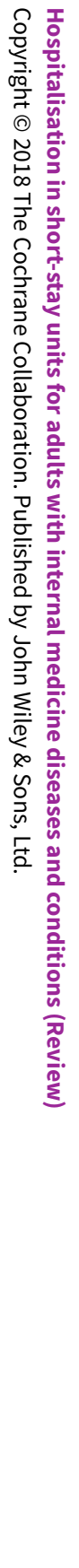 } & \multicolumn{7}{|c|}{$\begin{array}{l}\text { S U M M A R Y O F F I N D I N G S } \\
\text { Summary of findings for the main comparison. } \\
\text { conditions }\end{array}$} \\
\hline & \multicolumn{7}{|c|}{ Short-stay unit hospitalisation compared with usual care for internal medicine diseases and conditions } \\
\hline & \multicolumn{7}{|c|}{$\begin{array}{l}\text { Patient or population: participants with internal medicine diseases and conditions } \\
\text { Setting: hospitals (emergency department-based short-stay units in Denmark, New Zealand, Spain, UK, and US } \\
\text { Intervention: short-stay unit hospitalisation } \\
\text { Control: usual care }\end{array}$} \\
\hline & \multirow[t]{2}{*}{ Outcomes } & \multicolumn{2}{|c|}{ Anticipated absolute effects ${ }^{*}(95 \% \mathrm{Cl})$} & \multirow{2}{*}{$\begin{array}{l}\text { Relative effect } \\
(95 \% \mathrm{Cl})\end{array}$} & \multirow{2}{*}{$\begin{array}{l}\text { № of partici- } \\
\text { pants } \\
\text { (studies) }\end{array}$} & \multirow{2}{*}{$\begin{array}{l}\text { Certainty of } \\
\text { the evidence } \\
\text { (GRADE) }\end{array}$} & \multirow[t]{2}{*}{ Comments } \\
\hline & & Risk with us & $\begin{array}{l}\text { Risk with short-stay } \\
\text { unit hospitalisation }\end{array}$ & & & & \\
\hline & \multirow{2}{*}{$\begin{array}{l}\text { Mortality at } \\
\text { time point clos- } \\
\text { est to } 90 \text { days }\end{array}$} & \multicolumn{2}{|c|}{ Study population } & \multirow{2}{*}{$\begin{array}{l}\text { RR } 0.73 \\
(0.47 \text { to } 1.15)\end{array}$} & \multirow{2}{*}{$\begin{array}{l}1294 \\
(5 \mathrm{RTs})\end{array}$} & \multirow{2}{*}{$\begin{array}{l}\oplus \Theta \Theta \Theta \\
\text { Very lowa,b,c,d }\end{array}$} & \multirow{2}{*}{$\begin{array}{l}\text { We were able to pool } 5 \text { trials in the meta- } \\
\text { analysis. } 7 \text { additional trials reporting on } \\
1299 \text { participants reported no deaths in } \\
\text { either group. Data were missing in } 1 \% \text { of } \\
\text { participants. }\end{array}$} \\
\hline & & 62 per 1000 & $\begin{array}{l}45 \text { per } 1000 \\
\text { ( } 29 \text { to } 71)\end{array}$ & & & & \\
\hline & \multirow{2}{*}{$\begin{array}{l}\text { Serious ad- } \\
\text { verse events at } \\
\text { time point clos- } \\
\text { est to } 90 \text { days }\end{array}$} & \multicolumn{2}{|c|}{ Study population } & \multirow{2}{*}{$\begin{array}{l}\text { RR } 0.95 \\
\text { (0.59 to } 1.54)\end{array}$} & \multirow{2}{*}{$\begin{array}{l}1907 \\
\text { (7 RTs) }\end{array}$} & \multirow{2}{*}{$\begin{array}{l}\oplus \odot \odot \odot \\
\text { Very lowa,b,d,e }\end{array}$} & \multirow{2}{*}{$\begin{array}{l}\text { We were able to pool } 7 \text { trials in the meta- } \\
\text { analysis. } 1 \text { additional trial with } 108 \text { par- } \\
\text { ticipants reported no serious adverse } \\
\text { events in either group. Data were miss- } \\
\text { ing in } 1 \% \text { of participants. }\end{array}$} \\
\hline & & 77 per 1000 & $\begin{array}{l}74 \text { per } 1000 \\
\text { (46 to } 119)\end{array}$ & & & & \\
\hline & $\begin{array}{l}\text { Quality of life } \\
\text { at time point } \\
\text { closest to } 90 \\
\text { days }\end{array}$ & \multicolumn{2}{|c|}{$\begin{array}{l}1 \text { trial demonstrated higher quality-of-life scores } \\
\text { measured by SF- } 36 \text { among participants hospi- } \\
\text { talised in short-stay units. } \\
3 \text { trials reported little or no difference in quality-of- } \\
\text { life scores, using either } \\
\text { EuroQol-5 Domain, Quality of Well Being Scale, the } \\
\text { Syncope Functional Status Questionnaire, or Min- } \\
\text { nesota Living With Heart Failure Scale. }\end{array}$} & - & $\begin{array}{l}1029 \\
\text { (4 RTs) }\end{array}$ & $\begin{array}{l}\oplus \ominus \ominus \ominus \\
\text { Very lowb,c,f,g }\end{array}$ & $\begin{array}{l}\text { We did not pool data because none of } \\
\text { the trials reported quality of life using } \\
\text { the same measurement tool. Data were } \\
\text { missing in } 20 \% \text { of participants. }\end{array}$ \\
\hline & $\begin{array}{l}\text { Activities of } \\
\text { daily living at } \\
\text { time point clos- } \\
\text { est to } 90 \text { days }\end{array}$ & \multicolumn{2}{|c|}{$\begin{array}{l}1 \text { trial demonstrated a small improvement or no } \\
\text { difference in Lawton's Instrumental Activities } \\
\text { of Daily Living scores among participants hospi- } \\
\text { talised in short-stay units. }\end{array}$} & - & $\begin{array}{l}569 \\
\text { (2 RTs) }\end{array}$ & $\begin{array}{l}\oplus \odot \ominus \ominus \\
\text { Very lowb,c,f,g }\end{array}$ & $\begin{array}{l}\text { We were unable to pool data, because } \\
\text { none of the trials reported activities of } \\
\text { daily living using the same measure- } \\
\text { ment tool. Data were missing in } 19 \% \text { of } \\
\text { participants. }\end{array}$ \\
\hline
\end{tabular}

Patient or population: participants with internal medicine diseases and conditions

ing: hospitals (emergency departme

Control: usual care 


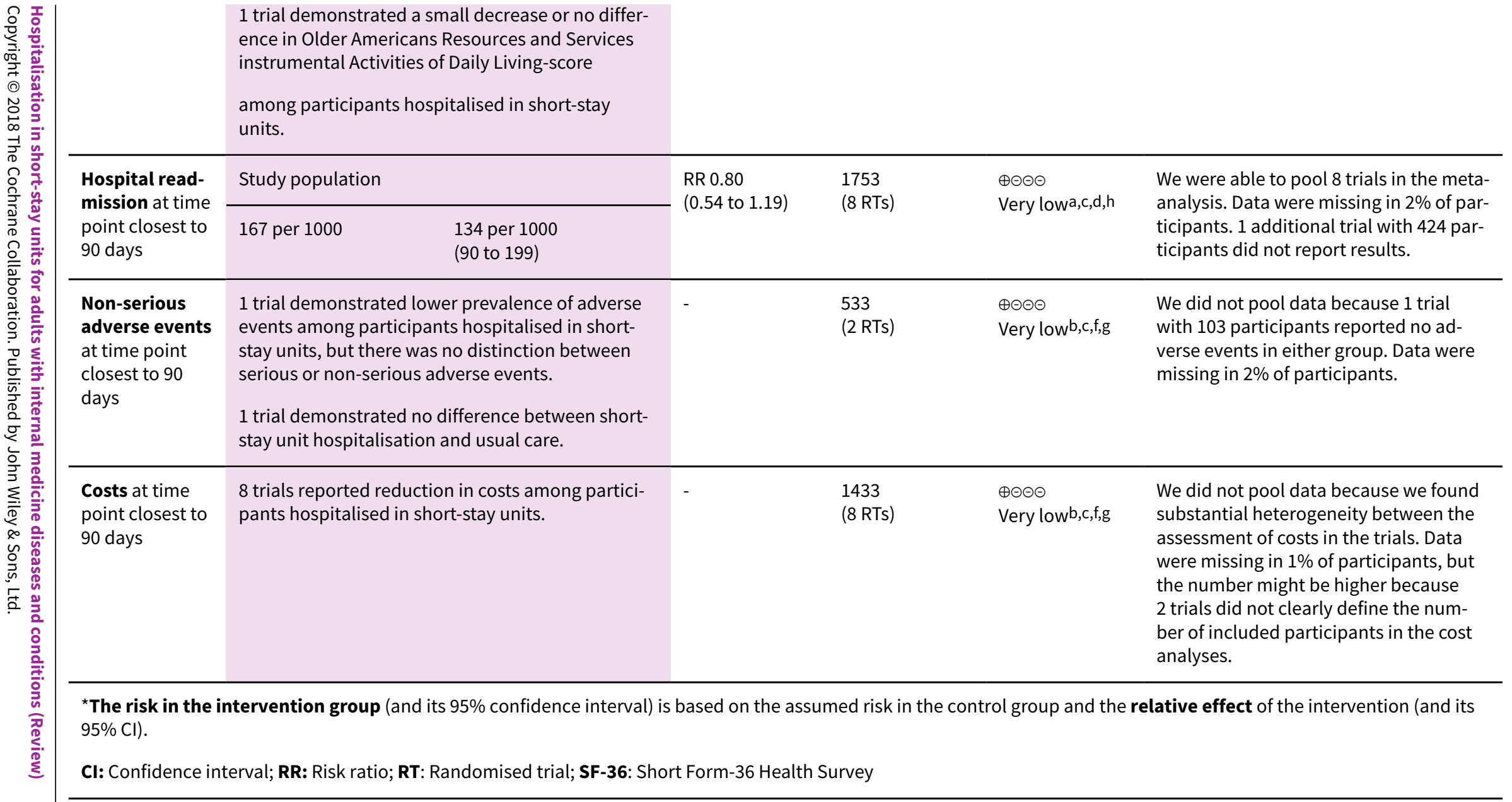

\section{GRADE Working Group grades of evidence}

High certainty: we are very confident that the true effect lies close to that of the estimate of the effect.

Moderate certainty: we are moderately confident in the effect estimate: the true effect is likely to be close to the estimate of the effect, but there is a possibility that it is substantially different.

Low certainty: our confidence in the effect estimate is limited: the true effect may be substantially different from the estimate of the effect.

Very low certainty: we have very little confidence in the effect estimate: the true effect is likely to be substantially different from the estimate of effect.

aDowngraded one level for serious risk of bias due to all trials being at high risk of bias, but because the outcome is a more objective outcome, lack of blinding of participants, personnel and outcome assessors may not bias the outcome as much.

bDowngraded one level for clinical heterogeneity among the included trials.

cNot downgraded for indirectness. 
Downgraded one level due to indirect evidence (surrogate outcome measures for adverse events)

Downgraded two levels due to all trials being at high risk of bias.

gNot downgraded for imprecision. We were not able to evaluate estimate of effect in meaningful meta-analysis.

howngraded two levels for clinical and statistical heterogeneity among the included trials. 


\section{B A C K G R O U N D}

Acute healthcare systems are under pressure. A steadily increasing demand for acute care delivery, decreasing number of acute care beds, and escalating healthcare costs have been observed worldwide (Lowthian 2011; WHO 2011). On a daily basis, both emergency departments and in-hospital services have to deal with hazardous patient overflow (Sprivulis 2006). One proposed solution to these challenges is to establish short-stay units (Salazar 2007). A short-stay unit is a hybrid ward that on one hand can offload stable patients from the emergency department or traditional wards for further investigations and risk stratification, and on the other hand can accommodate lower acuity patients, who need shortterm monitoring, observation, focused diagnostics, or therapeutic interventions (Galipeau 2015).

Short-stay units are believed to play a pivotal role in optimising the effectiveness of hospitals by streaming selected patients away from the traditional hospital ward services and expediting care for uncomplicated patients. Academic societies, such as The American College of Emergency Physicians specifically recommend implementation of short-stay units in emergency departments (ACEP 2008), and The European Society of Cardiology has recommended implementation of syncope short-stay units (Kenny 2015).

Several studies have indicated that short-stay units are capable of providing shorter hospital stays, reducing hospital readmission rates, lowering costs, and improving patient satisfaction (Arendts 2006; Farkouh 1997; Goodacre 2007; Roberts 1997; Ross 2013; Rydman 1997). However, other studies have questioned the positive findings by demonstrating inadequate efficiency (Russell 2014). This review set out to investigate whether short-stay unit hospitalisation is a viable alternative to usual care for patients with internal medicine diseases and conditions.

\section{Description of the condition}

This review focuses on adults with diseases or conditions within the spectrum of internal medicine, including conditions that are usually treated in internal medicine departments, for example, anaemia, asthma, cellulitis, chest pain, deep venous thrombosis, or urinary infections. Management of these patients can be challenging; patients can present with several non-specific symptoms from various organ systems and different diseases, both acute and chronic, and require complex investigations and multifaceted care, such as the involvement of different specialists, special nursing expertise, respiratory therapy, or physiotherapy. Accordingly, people with internal medicine diseases account for the majority of health care provided in hospitals. Worldwide, internal medicine diseases such as diabetes and cardiovascular disease are the leading cause of disability and death (CDC 2013; EFIM 2007; WHO 2011). Prevalences of chronic internal medicine diseases are rapidly rising; currently, one in two adults is affected by at least one chronic disease (Gerteis 2014; Pfuntner 2013; WHO 2011; Wolff 2002). This spreads into the acute care system because an increasing number of people need acute hospitalisation due to worsening of symptoms of a chronic disease (Dang-Tan 2015; WHO 2011). Additionally, infectious diseases such as pneumonia or urinary tract infections are still common reasons for acute hospitalisation (Christensen 2009, Pfuntner 2013; Weiss 2014). Infectious diseases continue to be associated with substantial morbidity and mortality (Christensen 2009; Lowthian
2011). Despite theoretical advances in sanitation and health care, the annual hospitalisation rate for infectious diseases has increased over recent decades. For example, the incidence has been estimated at 15 hospitalisations per 1000 people per year in the USA (Christensen 2009).

\section{Description of the intervention}

The intervention of interest is short-stay unit hospitalisation for internal medicine diseases and conditions. Since the 1960s, short-stay units have been introduced increasingly in Western countries (Cerce 1981; Galipeau 2015). The earliest models targeted paediatric and surgical patients (Laskin 1972). Subsequent models have targeted internal medicine patients (Daly 2003).

Numerous names for short-stay units can be found in the literature, such as observation unit, acute medical unit, medical assessment and planning unit, or quick diagnostic unit. There is no widelyaccepted definition of short-stay units, but their services are often defined by:

- a target patient category, for example, paediatric patients, adult patients with acute asthma, or surgical patients;

- a target function, for example, observation care, or interventions driven by protocols; and

- a target time frame of maximum stay in the unit, often set between 6 to 72 hours (Daly 2003; Damiani 2011; Galipeau 2015).

\section{Target patient category}

To ensure an optimal flow of patients, many short-stay units use strict admission and discharge criteria (Galipeau 2015; Gaspoz 1991). Specialised short-stay units accommodate a narrow and well-defined group of patients such as adult patients with chest pain or patients with acute exacerbation of asthma (Broquetas 2008; Jibrin 2008), while multipurpose short-stay units accommodate patients with a wide range of clinical symptoms and conditions (Arendts 2006; Strøm 2017a). Many short-stay units exclusively admit patients with minor medical ailments, for example, chronically ill patients needing blood transfusions, or acutely admitted patients with concussions in need of observation.

\section{Target function}

Many short-stay units are utilised to provide brief observation or diagnostic investigations for acute patients in order to make more appropriate disposition and management decisions (Mosely 2013). To alleviate emergency department crowding, many shortstay units serve as a buffer for the emergency departments by accommodating patients for initial triage and assessments (Galipeau 2015). Some also provide brief therapeutic interventions. Compared with usual care in an ordinary internal medicine ward, many short-stay units apply components that potentially streamline patient care, and accelerate the diagnostic process or rehabilitation time. Examples of elements of such fast-track care include early discharge planning; immediate access to laboratory tests, investigations, imaging, or standardised observation; and diagnostic or intervention protocols (Daly 2003; Galipeau 2015; Gaspoz 1991).

Often, patients admitted to a short-stay unit have received an evaluation upon arrival in an emergency department (physical exam, medical history, medications review) and an initial plan for salvage of acute symptoms (Juan 2006). In the short-stay 
unit, further observation, diagnostics and treatments are carried out (Downing 2008). To optimise treatment and early discharge, evaluation upon arrival in a short-stay unit often includes assessments of functional capacity and the need for support after discharge, and planning of out-of-hospital care for non-acute medical problems (Daly 2003; Galipeau 2015).

The units are a ward, bay, or a defined area located adjacent to or within a department. Some short-stay units function as separate entities, while others function as part of a larger department, often as a part of an emergency department (Daly 2003; Damiani 2011; Galipeau 2015). A short-stay unit can therefore be run either by dedicated house staff, hospitalists, or under the clinical governance of the emergency department staff. Short-stay units are usually equipped with emergency medical treatment facilities, and sometimes advanced diagnostic equipment, such as radiologic or laboratory facilities (Daly 2003; Miller 2010; Miller 2013).

\section{Target time frame}

To facilitate a high turnover of patients in short-stay units, many institutions use a target time frame of maximum stay in units. The limit is often set between 6 to 72 hours for emergency departmentbased units (Galipeau 2015).

\section{How the intervention might work}

Short-stay units are likely to work as an intervention because they may reflect a more efficient service design, result in less exposure to adverse events during hospitalisation and provide tailored care for selected patients.

Short-stay units may represent a more efficient service design by reducing the time spent in hospital for patients. This may lead to less exposure to treatment errors and hospital-acquired conditions, such as adverse events, or loss of functional capacity due to immobilisation. Adverse events during hospitalisation occur frequently (Brennan 1991). For example, medication errors, falls, delirium (Inouye 1990), or nosocomial infections (Baker 2004; Brennan 1991; Thomas 2000). Despite high hospital sanitation standards, hospitalised patients cannot be entirely isolated from harmful microbes; infections may spread to susceptible patients from other patients, healthcare staff, or contaminated equipment. Large population studies have estimated that $3 \%$ to $17 \%$ of all hospitalised patients experience an adverse event during an episode of hospitalisation, and adverse events are associated with substantial physical impairment and mortality (Baker 2004; Brennan 1991; Thomas 2000; Vries 2008). Moreover, adverse events are associated with prolonged length of stay in hospital (Classen 1997), but it is unclear to what extent adverse events lead to prolonged length of stay in hospital, or whether prolonged hospital stay increases the risk of an adverse event (Strøm 2017b). We hypothesise that minimising length of stay in hospital by shortstay unit hospitalisation results in less exposure to adverse events during hospitalisation.

Short-stay units are expected to possess cost-sparing properties due to the nature of the focused care model. Studies have indicated that short-stay units are able to reduce traditional hospital ward admission rates and lower expenditures without compromising the quality of care (Roberts 1997; Sun 2014). The number of hospital readmissions is often used as a quality indicator of hospital care. Studies have indicated that short-stay units can reduce hospital readmission rates (Damiani 2011; Decker 2008; Miller 2010;
Miller 2013). Furthermore, short-stay unit hospitalisation has been associated with higher patient satisfaction scores (Rydman 1997).

Optimising the diagnostic, treatment and rehabilitation processes in a short-stay unit may also improve patient outcomes. Tailored care with focused assessments or specific treatment protocols may enhance recovery for some patients. Many short-stay units incorporate components of accelerated care into patient care, in a manner similar to those studied in surgical populations, sometimes referred to as the 'enhanced recovery after surgery' programme (ERAS). ERAS is based on the application of standardised treatment protocols and certain fast-track elements, such as accelerated mobilisation, early removal of drains or tubes, and early discharge planning. Systematic reviews have found that ERAS seems to reduce morbidity rates, speed up recovery, and shorten the duration of hospital stay (Spanjersberg 2011; Wind 2006). However, the contribution of each of the ERAS programme components remains uncertain. We find it possible that patients with internal medicine diseases and conditions may benefit in a similar way from streamlined care in short-stay units. Prevention of prolonged bed rest may improve rehabilitation and prevent functional decline related to hospitalisation. Early discharge planning may facilitate faster and appropriate discharge processes (Daly 2003; Galipeau 2015).

\section{Why it is important to do this review}

Treatment of patients with internal medicine diseases and conditions accounts for the vast majority of healthcare expenditures (WHO 2011) and costs are expected to rise significantly (Cowling 2014), especially because the global population is dramatically ageing (UN 2013). There is an urgent need to explore how healthcare systems can successfully adapt to this challenge, while providing the best possible care for patients. Implementation of short-stay units may be one useful strategy to cope with the increasing demand for hospital care. Already, there has been a substantial growth in short-stay units (Salazar 2007). Patients, payers, and healthcare systems have great interest in knowing the benefits and harms of this model of care, but the evidence has been sparse. In a review more than a decade old, Daly and colleagues concluded that short-stay units had the potential to reduce patients' length of stay in hospital, improve the efficiency of emergency departments, and improve cost effectiveness (Daly 2003). Since then, two systematic reviews have proposed that treatment of internal medicine diseases and conditions in shortstay units may reduce inpatient mortality and length of stay in hospital without increasing hospital readmission rates (Damiani 2011; Scott 2009). Another systematic review described the effect of multipurpose short-stay units on emergency department overcrowding, and assessing the units' effectiveness and safety, as reported in trials conducted in countries with healthcare systems similar to Canada (Galipeau 2015). They found the evidence to be insufficient (Galipeau 2015). This review is necessary to assess the effectiveness of both specialised and multipurpose short-stay units across a number of patient-centred and resource outcomes.

\section{OB JECTIVES}

To assess beneficial and harmful effects of short-stay unit hospitalisation compared with usual care in people with internal medicine diseases and conditions. 


\section{METHODS}

\section{Criteria for considering studies for this review}

\section{Types of studies}

We included randomised trials assessing the effect of shortstay unit hospitalisation compared with usual care for internal medicine diseases and conditions irrespective of publication date, publication type and status, reported outcomes, and language. We excluded quasi-randomised trials.

\section{Types of participants}

Eligible participants were hospitalised adults (aged 18 years or above) receiving care for any internal medicine disease or condition, including conditions that are usually treated at internal medicine departments (such as cellulitis, chest pain, chronic obstructive pulmonary disease, dyspnoea, or pneumonia). Surgical, obstetric or gynaecological participants, participants with mental illnesses, and ambulatory participants were not included.

\section{Types of interventions}

\section{Experimental group}

The intervention was hospitalised treatment in a short-stay unit. As indicated in the 'Description of the intervention' section, there is no widely accepted definition of short-stay units. We defined a short-stay unit to be a hospital ward where the targeted length of stay in hospital for patients was five days or less. Prior to the review, we identified a list of different names of short-stay units by handsearching the literature (Appendix 1). We accepted all of these terms, but the list was not comprehensive and we accepted other relevant terms if the time limit of length of stay in the unit was described and fulfilled our definition. We included both multipurpose and specialised short-stay units and reported the details of each unit.

\section{Control group}

Usual care/hospitalisation as defined by trial authors (mainly hospitalisation in a non-short-stay unit, but other services could be included such as hospital-at-home).

\section{Types of outcome measures}

We assessed the following outcomes (in accordance with their classification in EPOC 2013a):

- patient-centred outcomes: mortality, activities of daily living, quality of life;

- adverse events or harms: serious adverse events, non-serious adverse events; and

- utilisation, coverage, or access: hospital readmission, length of stay in hospital, transfer to another department, costs.

We assessed all outcomes at two time points:

- the time point closest to 90 days after randomisation (this was the outcome of primary interest); and

- at maximum follow-up.

\section{Primary outcomes}

- Mortality; proportion of participants that died at any time and of any cause
- Serious adverse events; proportion of participants with one or more serious adverse events as defined by the International Committee of Harmonisation-Good Clinical Practice (ICH-GCP): "any untoward medical occurrence that resulted in death, was life threatening, required inpatient hospitalisation or prolongation of existing hospitalisation, resulted in persistent or significant disability/incapacity" (ICH-GCP 1997).

\section{Secondary outcomes}

- Quality of life; measured on any valid scale, such as the 36-item Short Form Health Survey (SF-36) (Ware 1992)

- Activities of daily living; measured on any valid scale, such as Lawton's Instrumental Activities of Daily Living score (iADL) (Lawton 1969)

- Hospital readmissions; proportion of participants who were readmitted to hospital after index hospitalisation

- Non-serious adverse events; proportion of participants with a registration of any untoward medical occurrence

- Transfer to another department; proportion of participants who were transferred to hospitalisation in another department after initial admission to either the short-stay unit or usual-care group unit/ward

- Total length of stay in hospital; time from admission to discharge of index hospital stay. We evaluated this outcome with caution, because short hospitalisation was an implicit aim of the intervention.

- Comparable costs; we compared costs in a narrative way.

Reporting of the outcomes listed above were not an inclusion criterion for the review. We included all outcomes at the time point closest to 90 days after randomisation in Summary of findings for the main comparison.

\section{Search methods for identification of studies}

The EPOC Information Specialist developed the search strategies in consultation with the review authors.

\section{Electronic searches}

We searched the following databases on 13 December 2017:

- Cochrane Central Register of Controlled Trials (CENTRAL; 2017 Issue 11) in the Cochrane Library;

- MEDLINE Ovid (including Epub Ahead of Print, In-Process \& Other Non-Indexed Citations and Versions) (1946 onwards)

- EmbaseOvid (1974 onwards)

- Health Technology Assessment Database (HTA; 2016, Issue 4) in the Cochrane Library

The Medline search was peer reviewed according to the PRESS checklist (Peer Review of Electronic Search Strategies) (McGowan 2016) by a second Cochrane Information Specialist prior to translating it for running on other databases. Search strategies are comprised of keywords and controlled vocabulary terms. We applied no language or time limits. We searched all databases from database start date to date of search.

We searched the Cochrane Database of Systematic Reviews (CDSR) and the Database of Abstracts of Reviews of Effects (DARE) for primary trials included in related systematic reviews. 


\section{Searching other resources}

We conducted grey literature searches to identify studies not indexed in the databases listed above.

We searched:

- International Clinical Trials Registry Platform (ICTRP), World Health Organization (WHO), (searched 17 December 2017);

- ClinicalTrials.gov, US National Institutes of Health (NIH) (ClinicalTrials.gov), (searched 17 December 2017);

- OpenGrey, (searched 17 December 2017);

- Grey Literature Report (New York Academy of Medicine) (www.greylit.org), (searched 17 December 2017);

- Health Services Research Projects in Progress (HSRProj) (NICHSR), (searched 17 December 2017).

We also:

- searched other web sources, such as the King's Fund library database (www.kingsfund.org.uk/library) and Google Scholar (scholar.google.com) (searched 17 December 2017);

- contacted authors of relevant trials and reviews to clarify reported information or seek unpublished results/data, (from 28 February 2017 to 17 December 2017);

- searched individual journals and conference proceedings (to 17 December 2017);

- conducted a forward citation search of the included trials using Science Citation Index via ISI Web of Science (pcs.webofknowledge.com), (to 17 December 2017);

- searched the reference lists of all included studies (to 17 December 2017);
- searched the reference lists of systematic reviews on short-stay unit hospitalisation found in the search (to 17 December 2017).

All search strategies used are provided in Appendix 2.

\section{Data collection and analysis}

We performed the review according to the review protocol (Strøm 2016), Cochrane's recommendations (Higgins 2011a), and the recommendations of Cochrane Effective Practice and Organisation of Care (EPOC 2013b). We used Review Manager 5 (RevMan 5) to perform the analyses (RevMan 2014).

\section{Selection of studies}

We downloaded all titles and abstracts retrieved by the electronic search to endnote (Endnote X7) and removed duplicates. We imported titles and abstracts to the web-based software platform, Covidence (Covidence 2015). Two review authors (CS, JS, or MF) independently assessed all titles and abstracts for eligibility. We excluded trials that did not obviously match the inclusion criteria (see trial screening template; Appendix 3). Two review authors (CS, JS, or MF) independently assessed full-text reports of the remaining trials and excluded any that clearly did not meet the eligibility criteria. If there were any disagreements, a third review author (JCJ) was asked to arbitrate. We collated multiple reports of the same trial so that each trial rather than each report was the unit of interest in the review. Trials that were thought likely to be relevant, but that were subsequently excluded, we listed in the 'Characteristics of excluded studies' table including the reason for their exclusion (EPOC 2013c). The selection process is demonstrated in a PRISMA flow diagram (Liberati 2009; Figure 1). 
Figure 1. PRISMA study flow diagram

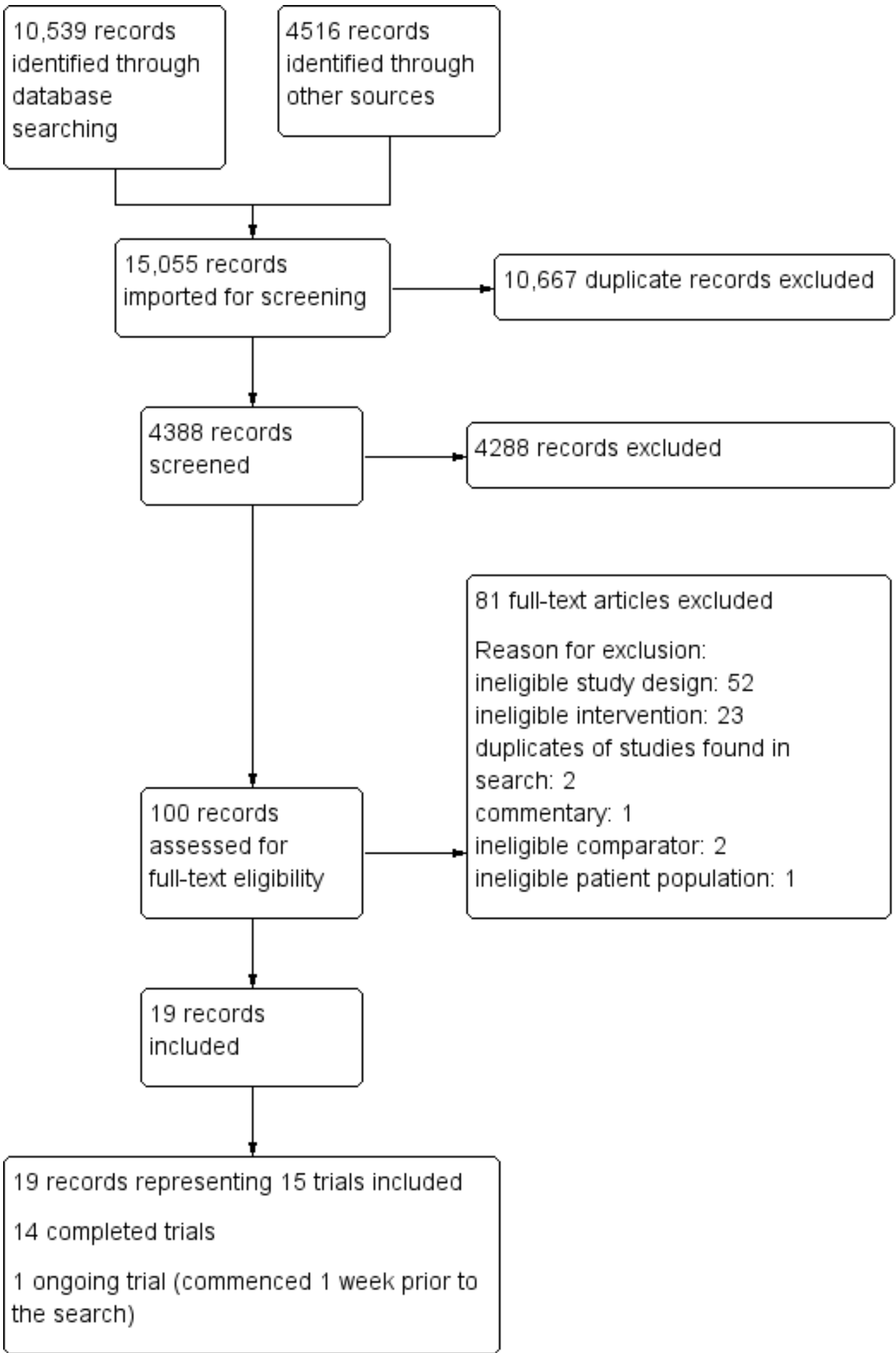




\section{Data extraction and management}

We used a modified EPOC data collection form (EPOC Supplementary materials) to capture trial characteristics and outcome data. Two review authors (CS, JS, or MF) extracted data independently, and validated data in pairs. Disagreements were resolved through involvement of a third review author (JCJ). We contacted trial authors for additional information and data as required. We noted in the 'Characteristics of included studies' tables if outcome data were reported in an unusable way.

\section{Assessment of risk of bias in included studies}

We assessed the risk of bias for all included trials in pairs (CS, JS, or MF) using the criteria outlined in chapter 8 of the Cochrane Handbook for Systematic Reviews of Interventions (Higgins 2011b), and the guidance from the EPOC Group (EPOC 2015a). We assessed all trials for allocation sequence generation, allocation concealment, baseline outcome measurement, baseline characteristics, blinding of outcome assessment, incomplete outcome data, contamination, selective outcome reporting, and other risk of bias. We considered it impossible for any trial to have blinded participants or treatment providers, and we chose not to include blinding of participants or treatment providers as bias domains.

We assessed each potential source of bias as high, low or unclear, and provided a justification for our judgment in a 'Risk of bias' table for each trial (see 'Characteristics of included studies'). Where information on the risk of bias related to unpublished data or correspondence with a trial author, we noted this in the 'Risk of bias' table.

We summarised our judgments in the 'Risk of bias' graph (Figure 2) and the 'Risk of bias' summary (Figure 3).

Figure 2. Risk of bias graph: review authors' judgements about each risk of bias item presented as percentages across all included trials.

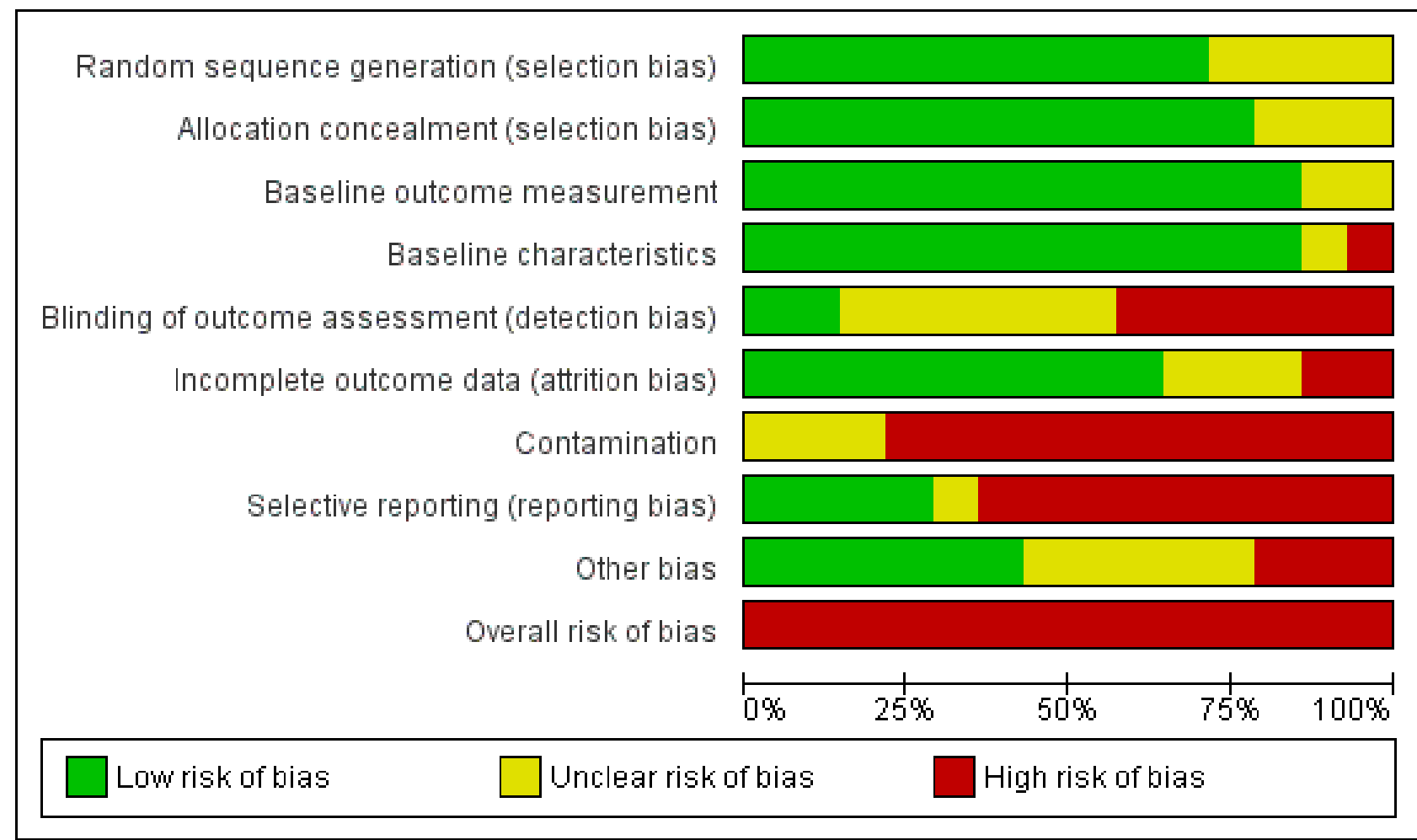


Figure 3. Risk of bias summary: review authors' judgements about each risk of bias item for each included trial.

\begin{tabular}{|c|c|c|c|c|c|c|c|c|c|c|}
\hline & 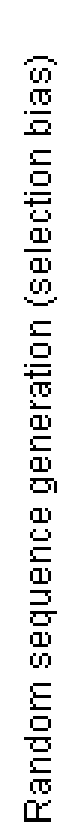 & 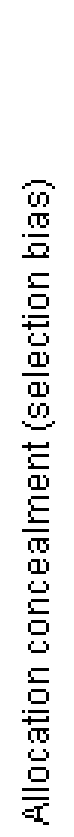 & 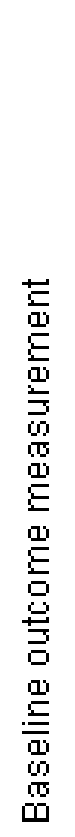 & 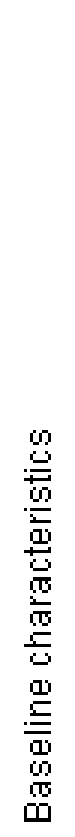 & 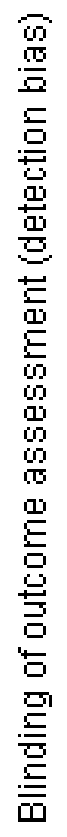 & 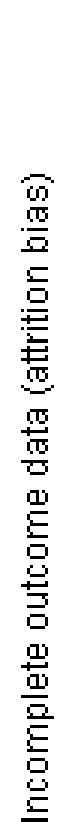 & 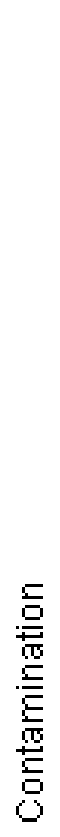 & 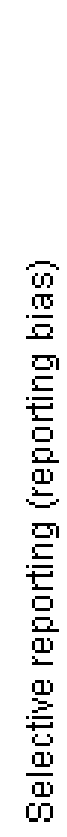 & 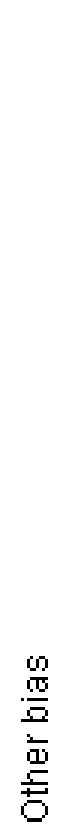 & 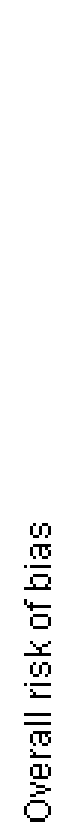 \\
\hline Chivite 2008 & + & + & $?$ & + & & & $?$ & & & \\
\hline Decker 2008 & + & + & + & $?$ & $?$ & + & & & + & \\
\hline Farkouh 1998 & ? & $?$ & + & + & $?$ & + & & & + & \\
\hline Gomez 1996 & $?$ & + & + & + & $?$ & + & & & 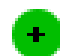 & \\
\hline McDermott 1997 & $?$ & $?$ & + & + & + & $?$ & $?$ & & $?$ & \\
\hline Miller 2010 & + & + & + & & & 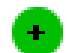 & & & & \\
\hline Miller 2013 & + & + & + & + & & & & & & \\
\hline Roberts 1997 & + & + & + & + & $?$ & + & & & $?$ & \\
\hline Ross 2007 & + & + & + & + & A & + & & & $?$ & \\
\hline Rydman 1997 & $?$ & $?$ & + & + & $?$ & + & $?$ & & + & \\
\hline Shen 2004 & + & + & + & + & & ? & & & & \\
\hline Strom 2017a & + & + & + & + & $?$ & + & & + & 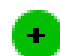 & \\
\hline $\operatorname{Sun} 2014$ & + & + & $?$ & . & & $?$ & & & $?$ & \\
\hline Than 2014 & + & + & + & + & + & + & & $?$ & $?$ & \\
\hline
\end{tabular}




\section{Measures of treatment effect}

\section{Dichotomous outcomes}

We calculated risk ratios (RR) and risk ratio differences together with $95 \%$ confidence intervals $(95 \% \mathrm{Cls}$ ).

\section{Continuous outcomes}

We planned to include both end scores and change scores and report the mean difference (MD) with $95 \% \mathrm{Cls}$ if all the trials used the same outcome scale, and standardised mean difference (SMD) with $95 \% \mathrm{Cls}$ when the trials measured the same continuous outcome, but used different scales.

\section{Minimal relevant clinical difference}

For each outcome, we predefined a minimal relevant clinical difference between the intervention and control group.

- Mortality: reduction or increase in risk ratio of $15 \%$

- Serious adverse event: reduction or increase in risk ratio of $20 \%$ (Baker 2004; Brennan 1991)

- Quality of life: a clinically relevant mean difference was equal to the observed standard deviation/2 (Jakobsen 2014)

- Activities of daily living: a clinically relevant mean difference was equal to the observed standard deviation/2 (Jakobsen 2014)

- Hospital readmission: reduction or increase in risk ratio of $20 \%$ (Miller 2013; Roberts 1997)

- Non-serious adverse events: reduction or increase of $20 \%$ in risk ratio.

- Transfer to other department: reduction or increase of $30 \%$ in risk ratio.

- Total length of stay in hospital: reduction or increase of $20 \%$ in risk ratio (Miller 2010; Miller 2013; Roberts 1997)

We did not report results as being statistically significant or nonsignificant (EPOC 2013f). Instead, we discussed the precision of the outcome estimates and considered whether the size of the effect was important, less important or not important according to the EPOC guidance (EPOC 2013b).

\section{Unit of analysis issues}

We planned to assess the effects of randomised trials and clusterrandomised trials separately using the generic inverse variance method according to chapter 9 of the Cochrane Handbook for Systematic Reviews of Interventions (Deeks 2011) to meta-analyse both types of trials in one analysis if possible. If the trial authors had not used appropriate methods to account for clustered data, we planned to follow the instructions in chapter 16 of the Cochrane Handbook for Systematic Reviews of Interventions to estimate 'effective sample sizes' (Higgins 2011c).

\section{Dealing with missing data}

We contacted investigators in order to obtain missing outcome data where possible (e.g. when a trial was identified as an abstract or if data were reported in an unusable way). Where possible, we analysed trials on an intention-to-treat basis. We did not impute missing values for any outcomes in our primary analysis. To explore the potential impact of missing data, we imputed data in order to conduct evaluation of best-worst and worst-best case scenarios (see Sensitivity analysis).

\section{Assessment of heterogeneity}

We investigated forest plots to visually assess heterogeneity. We tested statistical heterogeneity using the $\mathrm{Chi}^{2}$ test with a significance level set at $\mathrm{P} \leq 0.10$ and measured the quantities of heterogeneity using the 12 statistic (Deeks 2011; Higgins 2003).

\section{Assessment of reporting biases}

To reduce the risk of reporting bias, we undertook comprehensive searches of multiple databases and trials registries, and contacted trial authors to obtain missing information. We planned to create and examine funnel plots to explore possible publication biases if we were able to pool 10 trials or more in meta-analyses. If there were fewer than 10 trials available, we deemed publication bias to be non-assessable (Sterne 2011a).

\section{Data synthesis}

\section{Meta-analysis}

We performed both random-effects meta-analyses (DerSimonian 1986) and Mantel-Haenszel fixed-effect meta-analyses (Mantel 1959). We reported the more conservative point estimate of the two; that is, the estimate closest to zero effect (Jakobsen 2014), because the fixed-effect meta-analysis may show erroneous results if there is substantial statistical heterogeneity and the randomeffects meta-analysis may show erroneous results if one or two trials account for approximately $80 \%$ or more of the total weight in the meta-analysis (Jakobsen 2014). When estimates were equal, we used the estimate with the widest confidence interval $(\mathrm{Cl})$.

For the outcomes mortality, serious adverse events, and hospital readmission, we undertook meta-analyses at the time point closest to 90 days and at end of follow-up. For the outcomes: quality of life, activities of daily living, non-serious adverse events, transfer to another department, total length of stay in hospital, and costs, we were not able to conduct meaningful meta-analyses. Instead, we compiled narrative summaries.

\section{'Summary of findings' table and GRADE}

For the main intervention comparison (the outcomes as assessed at the time point closest to 90 days), three review authors in pairs (CS, JS, or MF) used the GRADE tool independently to assess the certainty of the evidence for each outcome (high, moderate, low, and very low) with respect to five criteria (inconsistency, indirectness, imprecision, publication bias, and risk of bias) (Guyatt 2008). We resolved disagreements through discussion. We used the GRADEpro GDT software and the recommendations from the Cochrane Handbook for Systematic Reviews of interventions (GRADEpro GDT 2015; Schünemann 2011). We prepared a 'Summary of findings' table (Summary of findings for the main comparison) of the most important outcomes including the justification for our decisions to downgrade the certainty of the evidence for an outcome, along with comments to help the reader understand the process (EPOC 2013d, EPOC 2013e).

\section{Subgroup analysis and investigation of heterogeneity}

We performed the following subgroup analysis on the outcomes mortality, serious adverse events, and hospital readmission for the comparison of the effect of short-stay unit hospitalisation compared with usual care, between trials investigating: 
- multipurpose short-stay units compared with specialised shortstay units (e.g. multipurpose short-stay unit, or dedicated chest pain short-stay unit);

- younger compared with older participants (participants were defined as 'older' either by the trial authors or they were aged over 65 years).

We regarded these findings to be observational. We used the test of interaction to analyse the test for subgroup differences (Deeks 2011; RevMan 2014).

We had planned to perform additional subgroup analyses but we were unable to due to a lack of data. We will perform these analyses in future updates of this review if data are available. See Differences between protocol and review for more details.

\section{Sensitivity analysis}

We performed sensitivity analyses defined a priori to assess the robustness of our conclusions and explore the impact on effect sizes. This involved:

- restricting the analyses to published trials;

- imputing missing data.

We analysed the impact of missing data by best-worst and worstbest case scenario analyses for dichotomous outcomes.

- 'best-worst case' scenario: we assumed that all participants lost to follow-up in the intervention group survived, had no serious adverse event, or were not readmitted. We assumed that all participants lost to follow-up in the usual-care group did not survive, had a serious adverse event, or were readmitted;

- 'worst-best case' scenario: we assumed that all participants lost to follow-up in the intervention group did not survive, had a serious adverse event, or were readmitted. We assumed that all participants lost to follow-up in the usual-care group survived, had no serious adverse event, or were not readmitted.

We did not conduct sensitivity analyses for continuous outcomes as planned. We also did not conduct sensitivity analyses as outlined in Assessment of risk of bias in included studies for trials with a low risk of bias, or for trials that evaluated outcomes at least once within six months of inclusion (i.e. six months of the participant being included in the trial). See Differences between protocol and review.

\section{RES ULTS}

\section{Description of studies}

We assessed all trials according to the Cochrane Handbook of Systematic Reviews of Interventions (Higgins 2011a), and the protocol for this review (Strøm 2016). Characteristics of each trial can be found in 'Characteristics of included studies' and 'Characteristics of excluded studies'.

\section{Results of the search}

We identified a total of 15,055 potentially relevant records from all sources searched. We removed 10,667 duplicates, and excluded 4288 records by the first screening process. We reviewed the full text of 100 records, of which one was a record found by forward citation. We obtained eight abstracts, 87 full reports, and 15 trial registrations, and a minimum of two review authors (CS, JS, or MF) independently reviewed them to assess eligibility. We identified one ongoing trial (NCT03302910). The reason for exclusion of trials and studies are described in Characteristics of excluded studies. The PRISMA flow diagram of the selection process is presented in Figure 1.

\section{Included studies}

We included 19 records reporting on 14 randomised trials in the review; one abstract, two trial registrations, and 16 full-text reports. One trial was ongoing. All but one trial were conducted between 1991 and 2016. We identified two unpublished trials. In one trial, the trial authors never sought to publish the results (Chivite 2008). The other trial was first accepted for publication after the search had been conducted; hence, we treated data as unpublished throughout the review (Strøm 2017a). The first author of this review was also the author of this trial, so all data extraction for this trial was performed by assessors not included in it (Strøm 2017a). Details on the included trials are listed in Characteristics of included studies; Table 1; and Table 2.

We contacted authors of all of the included trials to seek or clarify information. The authors of nine of the trials responded (Chivite 2008; Decker 2008; Gomez 1996; Miller 2010; Miller 2013; Shen 2004; Strøm 2017a; Sun 2014; Than 2014; Table 3).

\section{Settings}

Trials were undertaken in Denmark, New Zealand, Spain, UK, and USA. Eight were conducted at three institutions in the USA: three trials at The Mayo Clinic in Rochester, Minnesota (Decker 2008; Farkouh 1998; Shen 2004), three at Cook County Hospital in Chicago, Illinois (McDermott 1997; Roberts 1997; Rydman 1997), and two trials at Wake Forest Baptist Medical Centre in WinstonSalem, North Carolina (Miller 2010; Miller 2013). Trial paper publication dates ranged from 1996 to 2014 .

Eight of the trial reports were co-authored by one or more trial authors involved in more than one trial of short-stay units (Decker 2008; Farkouh 1998; McDermott 1997; Miller 2010; Miller 2013; Roberts 1997; Rydman 1997; Shen 2004).

\section{Target function}

All trials investigated emergency department-based short-stay units. Eight trials investigated multipurpose units (Chivite 2008; Decker 2008; Miller 2010; Miller 2013; Ross 2007; Strøm 2017a; Sun 2014; Than 2014), five trials investigated a specialised unit (Farkouh 1998; Gomez 1996; Roberts 1997; Rydman 1997; Shen 2004), and in one trial it was not clear whether the short-stay unit was specialised or not. The authors named the short-stay unit an "Emergency and Diagnostic Treatment Unit", and we assumed that this was a multipurpose unit (McDermott 1997).n 12 trials, the interventions were driven by treatment protocols (Decker 2008; Farkouh 1998; Gomez 1996; McDermott 1997; Miller 2010; Miller 2013; Roberts 1997; Ross 2007; Rydman 1997; Shen 2004; Sun 2014; Than 2014).

\section{Participants, experimental interventions and control interventions}

The 14 includedtrials randomised a total of 2872 participants (Chivite 2008; Decker 2008; Farkouh 1998; Gomez 1996; McDermott 1997; Miller 2010; Miller 2013; Roberts 1997; Ross 2007; Rydman 
1997; Shen 2004; Strøm 2017a; Sun 2014; Than 2014). The number of participants in each trial ranged from 100 to 544 participants.

\section{Target patient category}

One trial included participants with acute asthma (McDermott 1997), one included participants with atrial fibrillation (Decker 2008), seven included participants with chest pain (Farkouh 1998; Gomez 1996; Miller 2010; Miller 2013; Roberts 1997; Rydman 1997; Than 2014), one included participants with acute decompensated heart failure (Chivite 2008), two included participants with syncope (Shen 2004; Sun 2014), one included participants with transient ischaemic attack (Ross 2007), and one included participants with miscellaneous internal medicine disease and conditions (Strøm 2017a).

\section{Target time frame}

In total, seven trials used a maximum time frame as part of the intervention protocol (Decker 2008; McDermott 1997; Roberts 1997; Ross 2007; Rydman 1997; Shen 2004; Sun 2014). The maximum stay in the unit was set to six hours in one trial (Shen 2004), eight hours in one trial (Decker 2008), 12 hours in three trials (McDermott 1997; Roberts 1997; Rydman 1997), and 24 hours in two trials (Ross 2007; Sun 2014).

Five trials described a target maximum time frame, but the participants could occasionally have longer stays in the short-stay unit. Thus, the time frame was set to 16 hours in one trial (Than 2014), 24 hours in two trials (Miller 2010; Miller 2013), 72 hours in one trial (Strøm 2017a), and 120 hours in one trial (Chivite 2008).

The time frame was unclear in two trials (Farkouh 1998; Gomez 1996), but it appeared to be nine hours in one trial (Gomez 1996), because this was the time point for the last test that either led to discharge or admission to another hospital ward. The other trial merely said that participants were observed for a minimum of six hours in the short-stay unit (Farkouh 1998).

\section{Interventions}

The majority of the trials used a specific disease or condition as inclusion criteria and the components of the short-stay unit intervention were dictated by these conditions. Hence, we present below the characteristics of the randomised participants and the interventions by condition.

\section{Trials randomising participants with asthma}

Participants: the mean age of participants in the single trial including people with asthma was 36 years in the intervention group compared with 35 years in the usual-care group, and more men were included (men in short-stay unit versus usual-care group: $58 \%$ versus $64 \%$; McDermott 1997 ).

Interventions: one multicentre trial compared short-stay unit hospitalisation with inpatient care in participants with acute asthma (McDermott 1997). Participants randomised to shortstay unit hospitalisation received a standard-treatment protocol including scheduled therapy with inhaled bronchodilating agents and steroids, and repetitive clinical assessments. When participants met a set of predefined discharge criteria, they were discharged. The usual-care group received standard treatment according to national asthma guidelines in a hospital ward. Discharge criteria were identical to the intervention group, but participant assessments were only scheduled at time of arrival at the ward and on daily rounds (one round a day).

\section{Trials randomising participants with atrial fibrillation}

Participants: the mean age of participants in the single trial including people with atrial fibrillation was 58 years in the intervention group compared with 59 years in the usual-care group, and more men were included (men in short-stay unit versus usualcare group: 53\% versus 69\%; Decker 2008).

Interventions: one single-centre trial compared short-stay unit hospitalisation with inpatient care in participants with newly onset atrial fibrillation (Decker 2008). Participants randomised to short-stay unit hospitalisation received a standard care protocol including electrocardiogram recording, chest radiograph, routine laboratory investigations, pharmacologic pulse rate control, and continuous cardiac monitoring. If the condition had not resolved within six hours, participants underwent electrical cardioversion, and a further two hours of observation. Those remaining in atrial fibrillation after eight hours were admitted to the cardiology service. The usual-care group was treated conventionally at the cardiology service.

\section{Trials randomising participants with chest pain}

Participants: the mean age of participants in the seven trials that included people with chest pain ranged from 50 to 61 years in the intervention group compared with 50 to 64 years in the usual-care group. Slightly more men were included (range of proportion of men in short-stay unit versus usual-care group: $47 \%$ to 64\% versus 52\% to 69\%; Farkouh 1998; Gomez 1996; Miller 2010; Miller 2013; Roberts 1997; Rydman 1997; Than 2014). Three trials included participants who were stratified to have low probability of acute coronary syndrome (Gomez 1996; Roberts 1997; Rydman 1997), three trials included participants who were stratified to have intermediate or high probability of acute coronary syndrome (Farkouh 1998; Miller 2010; Miller 2013), and one trial did not use risk stratification as an inclusion criteria (Than 2014).

Interventions: in all seven trials, the intervention comprised 2 to 12 hours of observation in a short-stay unit, administration of aspirin, scheduled cardiac biomarker testing, and a cardiac stress test (e.g. treadmill testing) at the end of the observation period or scheduled to be conducted in an outpatient service within 72 hours (Farkouh 1998; Than 2014). Two trials incorporated up-front cardiac magnetic resonance imaging in the intervention group (Miller 2010; Miller 2013). Two trials used a standardised-care protocol for the usual-care group; all usual-care participants received scheduled biomarker testing and observation in traditional hospital ward services (Roberts 1997; Than 2014). For the remaining trials, allocation to the usual-care group warranted admission to coronary care, a telemetry unit, a cardiology service or general floor, and non-standardised treatment (Farkouh 1998; Gomez 1996; Rydman 1997) or usual-care procedures (Miller 2010; Miller 2013).

\section{Trials randomising participants with heart failure}

Participants: the mean age of participants in the single trial that included people with decompensated heart failure was 77 years in the intervention group compared with 79 years in the usual-care group, and slightly more men were included (men in short-stay unit versus usual-care group: $54 \%$ versus $61 \%$ ) (Chivite 2008 ). 
Interventions: one trial compared short-stay unit hospitalisation with hospitalisation in an internal medicine service for management of acute decompensated heart failure in older participants; an older person was defined as aged 65 years or older (Chivite 2008). It was a single-centre trial reported in abstract format.

\section{Trials randomising participants with syncope}

Participants: two trials randomised participants with syncope, stratified to be at 'intermediate' risk for an adverse cardiovascular outcome or a serious outcome. The mean age of participants ranged from 64 to 65 years in both groups, and the trials included equal proportions of men and women (range of proportion of men in short-stay unit versus usual-care group: $47 \%$ to $49 \%$ versus $48 \%$ to $52 \%$ ) (Shen 2004; Sun 2014).

Interventions: two trials compared short-stay unit hospitalisation with inpatient care for management of participants with syncope. One was a single-centre trial that compared hospitalisation in a short-stay unit with conventional care (Shen 2004). Participants in the intervention group underwent six-hour monitoring, scheduled orthostatic blood pressure measurements, tilt table test, carotid sinus massage and electrophysiologist consultation upon a physician's request. Participants with no indication for further hospitalisation were offered an outpatient follow-up consultation 72 hours after discharge. The other was a multicentre trial conducted at five hospitals that compared hospitalisation in a short-stay unit with standard hospital admission. Participants allocated to the intervention group received 12 to 24 hours of cardiac monitoring, scheduled biomarker testing and an echocardiogram (Sun 2014). In both of the trials, the usual-care groups received non-standardised care at the discretion of the responsible physicians (Shen 2004; Sun 2014).

\section{Trials randomising participants with transient ischaemic attack}

Participants: the mean age of participants in the single trial with people with transient ischaemic attach was 68 years in both groups, and there were more women than men (proportion of men in shortstay unit versus usual-care group: $41 \%$ versus $46 \%$ (Ross 2007 ).

Interventions: one, single-centre trial compared short-stay unit hospitalisation with inpatient care (primarily stroke unit, or internal medicine ward) for management of an episode of transient ischaemic attack (Ross 2007). Participants randomised to the intervention group received cardiac monitoring for at least 12 hours, carotid imaging, echocardiography, and serial clinical evaluations by nurses and emergency physicians, and a neurologist consultation. The usual-care group received non-standardised care at the discretion of the responsible physicians.

\section{Trials randomising participants with miscellaneous internal medicine} diseases or conditions

Participants: the mean age of participants in the single trial that did not define a specific disease or condition was 81 years in the intervention group compared with 82 years in the usual-care group, and more men were included in the intervention group (proportion of men in short-stay unit versus usual-care group: $47 \%$ versus $41 \%$ ) (Strøm 2017a).

Interventions: one single-centre trial compared short-stay unit hospitalisation with hospitalisation in an internal medicine department in participants aged 75 years or older (Strøm 2017a).
Participants randomised to the intervention group did not received a standardised treatment protocol, but discharge planning was initiated immediately after admission to the short-stay unit, and further diagnostic tests were performed on the same terms as in the emergency department, on a fast-track basis; including pointof-care ultrasonography, acute blood samples analysed in the emergency department's point-of-care laboratory, simple X-rays in the emergency department's X-ray room, and CT or MRI scans at the Department of Radiology. Participants were encouraged to mobilise as much as possible without assistance during the stay, which included getting minimal help to basic self-care activities such as bathing, getting out of bed, or walking around the department. The usual-care group was treated as usual at an internal medicine department.

For further details of each trial, see 'Characteristics of included studies'.

\section{Funding}

Thirteen trials described funding and financial support in detail (Table 1). Two trials were at risk of funding bias because a company with a potential financial interest in a certain result of the trials funded the trial authors (Miller 2010; Miller 2013).

\section{Excluded studies}

We excluded 81 trials and studies after full-text assessment based on our inclusion and exclusion criteria. Twenty-three trials did not assess short-stay unit hospitalisation as an intervention, 52 studies were excluded due to ineligible study design (two of these were quasi randomised trials randomising days not participants to shortstay unit care or usual care), one was a commentary, two trials used a ineligible comparator, one trial included an ineligible study population, and two reports turned out to be duplicates that were not identified in the primary selection process. For further details see 'Characteristics of excluded studies'.

\section{On-going studies}

We identified one ongoing study (NCT03302910). The trial was commenced one week prior to the date of the search; hence, no results were available for the current review. The trial is a multicentre trial comparing short-stay unit hospitalisation with usual care for treatment of acute heart failure. Participants are assigned to the short-stay unit for a treatment and observation period. Participants in both the short-stay unit and usual care arm receive usual medical care for acute heart failure, which includes loop diuretics and nitroglycerine, as needed. The timeframe for maximum stay in the trial is set to 23 hours (NCT03302910).

\section{Risk of bias in included studies}

\section{Allocation}

We assessed the generation of the allocation sequence generation as low risk of bias in 10 trials (Chivite 2008; Decker 2008; Miller 2010; Miller 2013; Roberts 1997; Ross 2007; Shen 2004; Strøm 2017a; Sun 2014; Than 2014). Four trials described themselves as being randomised, but the methods used for sequence generation were unclear (Farkouh 1998; Gomez 1996; McDermott 1997; Rydman 1997), so we judged these trials to be of unclear risk of bias for this domain. We converted one trial from having unclear risk of bias to having low risk of bias in this domain after contact with the trial authors (Chivite 2008). 
The method used to conceal allocation was adequate in 11 trials (Chivite 2008; Gomez 1996; Decker 2008; Miller 2010; Miller 2013; Roberts 1997; Ross 2007; Shen 2004; Strøm 2017a; Sun 2014; Than 2014), so we judged these trials to be at low risk of bias for this domain. The remaining three trials either did not describe the method used for allocation concealment or insufficiently described it (Farkouh 1998; McDermott 1997; Rydman 1997), and we judged these trials to be at unclear risk of bias for the domain. We converted one trial from having unclear risk of bias to having low risk of bias in this domain after contact with the trial authors (Chivite 2008).

\section{Baseline outcome measurement}

We assessed baseline outcome measurement as low risk of bias in 12 trials (Decker 2008; Farkouh 1998; Gomez 1996; McDermott 1997; Miller 2010; Miller 2013; Roberts 1997; Ross 2007; Rydman 1997; Shen 2004; Strøm 2017a; Than 2014), because they measured relevant performance or patient outcomes prior to the intervention and no important differences were present across trial groups, or baseline outcome measurement was irrelevant for all trial outcomes. Two trials did not specify any information on baseline outcome measurement (Chivite 2008; Sun 2014), and we judged the trials to have unclear risk of bias for this domain.

\section{Baseline characteristics}

We assessed baseline characteristics as low risk of bias in 12 trials (Chivite 2008; Gomez 1996; Farkouh 1998; McDermott 1997; Miller 2010; Roberts 1997; Ross 2007; Rydman 1997; Shen 2004; Strøm 2017a; Sun 2014; Than 2014). We judged one trial to be at high risk of bias for this domain (Miller 2010), because characteristics were imbalanced (cardiac events prior to hospitalisation were unevenly distributed), and one trial at unclear risk of bias (Decker 2008).

\section{Blinding}

Risk of performance bias was present in all 14 trials (Chivite 2008; Decker 2008; Farkouh 1998; Gomez 1996; McDermott 1997; Miller 2010; Miller 2013; Roberts 1997; Ross 2007; Rydman 1997; Shen 2004; Strøm 2017a; Sun 2014; Than 2014), because it was impossible to blind participants and healthcare personnel due to the nature of the intervention.

We assessed the blinding of outcome assessors as low risk of bias in two trials (McDermott 1997; Than 2014). The methods for blinding of outcome assessors for the remaining trials were either inadequate (Chivite 2008; Miller 2010; Miller 2013; Ross 2007; Shen 2004; Sun 2014) - we judged these trials to be at high risk for this domain - or insufficiently described (Decker 2008; Farkouh 1998; Gomez 1996; Roberts 1997; Rydman 1997; Strøm 2017a) - we judged these trials to be at high risk for this domain.

\section{Incomplete outcome data}

We assessed trials' handling of incomplete outcome data as low risk of bias in nine trials (Decker 2008; Farkouh 1998; Gomez 1996; Miller 2010; Roberts 1997; Ross 2007; Rydman 1997; Strøm 2017a; Than 2014). We judged three trials to have unclear risk of bias in this domain (McDermott 1997; Shen 2004; Sun 2014). Two trials either did not describe missing data or provided insufficient detail about how they dealt with them (McDermott 1997; Shen 2004)

The remaining trials were judged to be at high risk of bias for this domain (Chivite 2008; Miller 2013). Two trials were stopped early
(Miller 2013; Shen 2004). One trial did not calculate a sample size but included consecutive participants until the end of the funding (Chivite 2008). Numbers of participants lost to follow-up in Chivite 2008 varied depending on the outcome in that trial.

\section{Contamination}

We did not assess any of the trials to be at low risk of bias for contamination. We judged three trials to be at unclear risk of bias for this domain (Chivite 2008; McDermott 1997; Rydman 1997).

\section{Selective reporting}

We assessed selective outcome reporting as low risk of bias in four trials (Miller 2010; Miller 2013; Strøm 2017a; Sun 2014). One trial paper did not report upon the outcomes stated in the protocol (Than 2014), outcome details were provided after correspondence with the trial authors and we judged the trial to be at unclear risk of bias. We were unable to obtain the trial registration or protocol for nine trials that were all conducted before trial registration became mandatory in 2005 (Chivite 2008; Decker 2008; Farkouh 1998; Gomez 1996; McDermott 1997; Roberts 1997; Ross 2007; Rydman 1997; Shen 2004); we judged these trials to be at high risk of bias for this domain.

\section{Other potential sources of bias}

Seven trials had no other biases, resulting in 'low risk of other bias' (Decker 2008; Farkouh 1998; Gomez 1996; Rydman 1997; Shen 2004; Strøm 2017a;Than 2014). Three trials were judged to be at 'high risk of other bias', In two trials, the authors were industryfunded (Miller 2010; Miller 2013). In one trial, no sample size was calculated, consecutive participants were included until the end of the funding (Chivite 2008). The remaining trials we judged to be at 'unclear risk of other bias' (McDermott 1997; Roberts 1997; Ross 2007; Sun 2014).).

\section{Overall risk of bias}

Based on our predefined 'Risk of bias' assessment and the information that we collected from the published reports and from trial authors, we considered all 14 trials at high risk of bias overall. We judged many trials to be at unclear risk of bias in several domains. For review authors' judgements about each risk of bias item presented as percentages across all included trials see Figure 2 , and for review authors' judgements about each 'Risk of bias' item for each included trial see Figure 3.

\section{Effects of interventions}

See: Summary of findings for the main comparison Short-stay unit hospitalisation compared with usual care for internal medicine diseases and conditions

\section{Primary outcomes}

\section{Mortality}

\section{Time point closest to 90 days}

Twelve trials with a total of 2619 participants reported mortality at the time point closest to 90 days (Chivite 2008; Decker 2008; Farkouh 1998; Gomez 1996; McDermott 1997; Miller 2010; Miller 2013; Roberts 1997; Shen 2004; Strøm 2017a; Sun 2014; Than 2014). Nine trials reported data based on an intention-to-treat population (Decker 2008; Farkouh 1998; Gomez 1996; McDermott 1997; Miller 2010; Miller 2013; Roberts 1997; Shen 2004; Sun 2014). In 26 of 
2619 participants (1\%), information on mortality was missing or participants were lost to follow-up (Chivite 2008; Strøm 2017a; Than 2014). In total, 29 of 1290 short-stay unit participants (2\%), died compared with 40 of 1303 usual-care participants (3\%). Data including the specific assessment time points closest to 90 days in each trial are presented in Table 4.

We were able to pool only five trials in the meta-analysis (Chivite 2008; Farkouh 1998; McDermott 1997; Shen 2004; Strøm 2017a), because seven trials reported no deaths in either group (Decker 2008; Gomez 1996; Miller 2010; Miller 2013; Roberts 1997; Sun 2014; Than 2014). It is uncertain whether there is any difference in mortality at 90 days for participants hospitalised in short-stay units compared to usual care (RR $0.73,95 \% \mathrm{Cl} 0.47$ to 1.15 ; participants $=1294 ;$ studies $=5 ; 1^{2}=0 \%$, very low-certainty evidence, randomeffects meta-analysis; Analysis 1.1). Neither visual inspection of the forest plots nor the tests for statistical heterogeneity $\left(I^{2}=0 \% ; P=\right.$ 0.18 ) indicated significant heterogeneity. Publication bias was not assessable (fewer than 10 trials in the meta-analysis).

The best-worst and worst-best case meta-analyses showed that incomplete outcome data bias alone had the potential to influence the results. Best-worst random-effects meta-analysis: RR 0.57 (95\% $\mathrm{Cl} 0.37$ to 0.87 ; participants $=1318$; studies $=5 ; 12=0 \%$, very lowcertainty evidence; Analysis 1.2), and worst-best random-effects meta-analysis: RR $1.05(95 \% \mathrm{Cl} 0.57$ to 1.94 ; participants $=1318$; studies $=5 ; 1^{2}=16 \%$, very low-certainty evidence; Analysis 1.3$)$. We imputed data for two trials (Chivite 2008; Strøm 2017a). None of the additional analyses showed evidence of important influence of the investigated factors (trial publication status, time point of outcome assessment; Analysis 1.4; Analysis 1.5) or subgroup differences (Analysis 1.6; Analysis 1.7; Analysis 1.8).

Using GRADE, we assessed the certainty of the evidence to be very low for mortality. We considered mortality to be an objective outcome at low risk of bias, i.e., lack of blinding of participants, personnel and outcome assessors may not bias the outcome as much. However, we downgraded the outcome one level for risk of bias due to all the trials being at high risk of bias in the GRADE assessment. The reason for the GRADE judgement is outlined in Appendix 4 and in Summary of findings for the main comparison.

\section{Time point at maximum follow-up}

Twelve trials with a total of 2619 participants reported mortality at maximum follow-up (Chivite 2008; Decker 2008; Farkouh 1998; Gomez 1996; McDermott 1997; Miller 2010; Miller 2013; Roberts 1997; Shen 2004; Strøm 2017a; Sun 2014; Than 2014). Seven trials reported data based on an intention-to-treat population (Decker 2008; Gomez 1996; McDermott 1997; Miller 2013; Roberts 1997; Shen 2004; Sun 2014). In a total of 44 of 2619 participants (2\%), information on mortality was missing or participants were lost to follow-up (Chivite 2008; Farkouh 1998; Miller 2010; Strøm 2017a; Than 2014). A total of 66 of 1281 short-stay unit participants (5\%) died compared with 79 of 1294 usual-care participants (6\%). Data including the specific assessment time points in each trial are presented in Table 5.

We were only able to pool five of the 12 trials in the metaanalysis for the same reasons stated in the 90-day analysis (Chivite 2008; Farkouh 1998; McDermott 1997; Shen 2004; Strøm 2017a). It is uncertain whether there is any difference in mortality at maximum follow-up for participants hospitalised in short-stay units compared to usual care (RR $0.84,95 \% \mathrm{Cl} 0.62$ to 1.13 ; participants $=1277$; studies $=5 ; 1^{2}=0 \%$, very low-certainty evidence, randomeffects meta-analysis; Analysis 1.9). Neither visual inspection of the forest plots nor tests for statistical heterogeneity $(\mathrm{I}=0 \%$; $\mathrm{P}=$ 0.80 ) indicated significant heterogeneity. Publication bias was not assessable (fewer than 10 trials in the meta-analysis).

The best-worst and worst-best case meta-analyses showed that incomplete outcome data bias alone had the potential to influence the results. Best-worst random-effects meta-analysis: RR 0.67 (95\% $\mathrm{Cl} 0.50$ to 0.88 ; participants $=1318$; studies $=5 ; \mathrm{I}^{2}=0 \%$, very low-certainty evidence; Analysis 1.10), and worst-best randomeffects meta-analysis: RR $1.17(95 \% \mathrm{Cl} 0.73$ to 1.89 ; participants = 1318 ; studies $=5 ; 1^{2}=35 \%$, very low-certainty evidence; Analysis 1.11). We imputed data for three trials (Chivite 2008; Farkouh 1998; Strøm 2016). None of the additional analyses showed evidence of important influence of the investigated factors (trial publication status, time point of outcome assessment; Analysis 1.12; Analysis 1.13) or subgroup differences (Analysis 1.14; Analysis 1.15; Analysis 1.16).

\section{Serious adverse events}

None of the trials used the exact term 'serious adverse events'. However, the trials reported a number of composite outcomes that we propose reflect serious adverse events according to the ICH-GCP definition (ICH-GCP 1997). One trial that recruited participants with atrial fibrillation reported a composite outcome of acute myocardial infarction, heart failure, stroke, or death (Decker 2008). A trial that recruited participants with chest pain used similar criteria, but added out-of-hospital cardiac arrest as a criterion (Farkouh 1998). Two other trials that recruited participants with chest pain were conducted by the same research group (Miller 2010; Miller 2013). In Miller 2010, trial authors reported major cardiac events defined by any of the following: myocardial infarction, revascularization, or cardiovascular death. In Miller 2013, trial authors reported safety events defined to be either death, acute coronary syndrome after discharge, or adverse events of cardiac stress testing. A further trial that recruited chest pain participants used the term 'major adverse cardiac event' to cover any of the following: cardiac death, cardiac arrest, emergency revascularization procedure, cardiogenic shock, ventricular arrhythmia needing intervention, high-degree atrioventricular block needing intervention, or myocardial infarction (Than 2014). In a trial that recruited participants with transient ischaemic attack, trial authors used the recorded subsequent stroke within 90 days, major clinical events (seizures, foramen ovale closure etc.), and major adverse cardiac events (major dysrhythmia, new myocardial infarction, cardiac arrest, revascularisation, new congestive heart failure, cardiac death; Ross 2007). A trial that recruited participants with syncope reported serious clinical events, defined to be any of the following: death, ventricular arrhythmia, heart block, sick sinus syndrome, sinus pause greater than three seconds, symptomatic supraventricular tachycardia, symptomatic bradycardia, major cardiac intervention, myocardial infarction, stroke, pulmonary embolism, aortic dissection, non-traumatic intracranial haemorrhage, internal haemorrhage or anaemia requiring transfusion, and major traumatic injury associated with syncope, near-syncope or fall (Sun 2014). One trial that recruited older participants with miscellaneous internal medicine diseases 
and conditions reported deaths during the index hospital stay (Strøm 2017a).

\section{Time point closest to 90 days}

Eight trials with a total of 2039 participants reported serious adverse events at the time point closest to 90 days (Decker 2008; Farkouh 1998; Miller 2010; Miller 2013; Ross 2007; Strøm 2017a; Sun 2014; Than 2014). Four trials reported data based on an intentionto-treat population (Decker 2008; Farkouh 1998; Miller 2013; Ross 2007). In a total of 24 of 2039 participants (1\%), information regarding serious adverse events was missing or participants were lost to follow-up (Miller 2010; Strøm 2017a; Sun 2014; Than 2014). A total of 76 of 1002 short-stay unit participants (8\%) had a serious adverse event compared with 74 of 1013 of usual-care participants (7\%). Data including the specific assessment time points in each trial are presented in Table 6.

We were able to pool seven of the eight trials in the metaanalysis (Decker 2008; Farkouh 1998; Miller 2013; Ross 2007; Strøm 2017a; Sun 2014; Than 2014); Miller 2010 reported zero serious adverse events. It is uncertain whether there is any difference in serious adverse events at 90 days for participants hospitalised in short-stay units compared to usual care (RR 0.95, 95\% Cl 0.59 to 1.54 ; participants $=1907$; studies $=7 ; 1^{2}=34 \%$, very lowcertainty evidence, random-effects meta-analysis; Analysis 2.1). The visual inspection of the forest plots and the tests for statistical heterogeneity $(12=34 \% ; \mathrm{P}=0.16)$ indicated moderate statistical heterogeneity; hence, the included trials in the SAE meta-analysis seem to show different intervention effect estimates. This might be cause by differences in the trial interventions, participant characteristics, different co-interventions, definitions of serious adverse events etc. in the included trials. There is a risk that pooling these trials in a meta-analysis for serious adverse events may produce a misleading result.

Investigation of the forest plots showed that one trial alone could have accounted for the statistical heterogeneity. When we removed Farkouh 1998, heterogeneity was also removed, both visually and statistically (RR $1.23,95 \% \mathrm{Cl} 0.88$ to 1.72 ; participants = 1483; studies $=6 ; 1^{2}=0 \%$; Analysis 2.18). Publication bias was not assessable (fewer than 10 trials in the meta-analysis).

The best-worst and worst-best case meta-analyses showed that incomplete outcome data bias did not have the potential to influence the results. Best-worst random-effects meta-analysis: RR $0.79\left(95 \% \mathrm{Cl} 0.50\right.$ to 1.26 ; participants $=1929$; studies $=7 ;\left.\right|^{2}=37 \%$, very low-certainty evidence; Analysis 2.2 ), and worst-best randomeffects meta-analysis: RR $1.13(95 \% \mathrm{Cl} 0.67$ to 1.89 ; participants = 1929 ; studies $=7 ; 1^{2}=46 \%$, very low-certainty evidence; Analysis 2.3). We imputed data for three trials (Strøm 2017a; Sun 2014; Than 2014). None of the additional analyses showed evidence of important influence of the investigated factors (trial publication status, time point of outcome assessment; Analysis 2.4; Analysis 2.5) or subgroup differences (Analysis 2.6; Analysis 2.7; Analysis 2.8).

Using GRADE, we assessed the certainty of the evidence to be very low for serious adverse events. We considered serious adverse events to be an objective outcome at low risk of bias. However, we downgraded the outcome one level for risk of bias due to all the trials being at high risk of bias in the GRADE assessment. The reason for the GRADE judgement is outlined in Appendix 4 and Summary of findings for the main comparison.

\section{Time point at maximum follow-up}

In total, eight trials with a total of 2039 participants reported serious adverse events at maximum follow-up (Decker 2008; Farkouh 1998; Miller 2010; Miller 2013; Ross 2007; Strøm 2017a; Sun 2014; Than 2014). Three trials reported data based on an intention-to-treat population (Decker 2008; Miller 2013; Ross 2007). In a total of 51 of 2039 participants (3\%), information regarding serious adverse events was missing or participants were lost to follow-up (Farkouh 1998; Miller 2010; Strøm 2017a; Sun 2014; Than 2014). A total of 117 of 989 short-stay unit participants $(12 \%)$ had a serious adverse event compared with 108 of 999 usual-care participants (11\%). Maximum follow-up ranged from length of the stay in hospital to an observation-time median of 5.5 years (interquartile range (IQR) 4.8 to 6 years) among trials. Data including the specific assessment time points at maximum follow-up in each trial are presented in Table 7.

We were able to pool data from all eight trials (Decker 2008; Farkouh 1998; Miller 2010; Miller 2013; Ross 2007; Strøm 2017a; Sun 2014; Than 2014). It is uncertain whether there is any difference in serious adverse events at maximum follow-up for participants hospitalised in short-stay units compared to usual care (random-effects metaanalysis: RR 1.11, 95\% Cl 0.87 to 1.41; participants $=1988$; studies = $8 ; 1^{2}=0 \%$, very low-certainty evidence; Analysis 2.9). Neither visual inspection of the forest plots nor tests for statistical heterogeneity $(12=0 \% ; P=0.73)$ indicated significant heterogeneity. Publication bias was not assessable (fewer than 10 trials in the meta-analysis).

The best-worst and worst-best case meta-analyses showed that incomplete outcome data bias had the potential to influence the results. Best-worst random-effects meta-analysis: RR $0.83(95 \% \mathrm{Cl}$ 0.60 to 1.16 ; participants $=2039$; studies $=8 ;\left.\right|^{2}=30 \%$, very lowcertainty evidence; Analysis 2.10), and worst-best random-effects meta-analysis: RR $1.35(95 \% \mathrm{Cl} 1.07$ to 1.70 ; participants = 2039; studies = 8; $\left.\right|^{2}=0 \%$, very low-certainty evidence; Analysis 2.11). We imputed data for five trials (Farkouh 1998; Miller 2010; Strøm 2017a; Sun 2014; Than 2014). None of the additional analyses showed evidence of important influence of the investigated factors (trial publication status, time point of outcome assessment; Analysis 2.12; Analysis 2.13) or subgroup differences (Analysis 2.14; Analysis 2.15; Analysis 2.16).

\section{Secondary outcomes}

\section{Quality of life}

\section{Time point closest to 90 days}

Four trials with a total of 1029 participants recorded quality of life at the time point closest to 90-days (Chivite 2008; McDermott 1997; Sun 2014; Than 2014). None of the trials reported results based on an intention-to-treat population. Data were missing in 206 of 1029 participants (20\%). Data and a further description of scoring systems are presented in Table 8.

None of the trials reported quality of life using the same measurement tool, hence we could not pool data in meta-analyses.

Chivite 2008 reported quality of life using the Minnesota Living With Heart Failure Scale in 59 of 70 participants (84\%) in the intervention group and in 59 of 69 participants (86\%) in the usual-care group. Participants in the intervention group had marginally higher mean scores at three months; mean 23 (SD 9) in the intervention group compared with 22 (SD 9) in the usual-care group. 
McDermott 1997 reported quality of life using The Short Form-36 Health Survey (SF-36). The trial reported all the eight separate domains of the SF-36 for a subgroup of participants (113/222 $(51 \%))$, it was not clear how the subgroup had been selected. Trial authors reported that participants in the intervention group had significantly better outcomes in the physical, emotional role, social functioning, mental health, and vitality domains at seven days after randomisation (Mean SF-36 domains in intervention group versus usual-care groups with P-values: physical functioning 72 (27.43) versus 58 (29.04), $\mathrm{P}=0.01$; emotional functioning role 78 (51.31) versus 45 (44.62), $P=0.001$; social functioning 80 (27.95) versus 68 (29.27), $\mathrm{P}=0.02$; mental health 78 (19.44) versus 67 (26.00), $\mathrm{P}=$ 0.008 , and vitality 59 (22.99) versus 47 (25.73), $\mathrm{P}=0.02$ ).

Sun 2014 reported quality of life using change in Quality of Well Being Scale and the Syncope Functional Status Questionnaire in 46 of 62 participants (74\%) in the intervention group and in 41 of 62 participants (66\%) in the usual-care group. The MD in Quality of Well Being score was $-0.02(95 \% \mathrm{Cl}-0.10$ to 0.06$)$ and MD in depression score was $-5.2(95 \% \mathrm{Cl}-15.2$ to 4.8$)$, in favour of the intervention group.

Than 2014 reported quality of life using the EuroQol-5 Domain (EQ5D). Trial authors did not include quality of life in the primary publication, but stated that 253 of 270 participants (94\%) in the intervention group compared with 250 of 272 participants (92\%) in the usual-care group completed an EQ5D at three months after inclusion. The mean scores in the intervention group versus the usual-care group were: 0.728 (standard deviation (SD) 0.09) versus 0.716 (SD 0.10).

Publication bias was not assessable. Using GRADE, we assessed the certainty of the evidence to be very low for quality of life. We considered this outcome at high risk of bias. The reason for the GRADE judgement is outlined in Appendix 4 and Summary of findings for the main comparison.

\section{Time point at maximum follow-up}

One of the above mentioned trials (in 'Quality of life time point closest to 90 days') obtained quality of life at a longer-term followup (Chivite 2008) for 48 of 70 participants (69\%) in the intervention group and for 47 of 69 participants $(68 \%)$ in the usual-care group. The trial reported quality of life at 12 months, using the Minnesota Living With Heart Failure Scale. Participants in the two groups had equal mean scores at 12 months; mean 22 (SD 12) in the intervention group compared with 22 (SD 12) in the usual-care group. Data were missing in 44 of 139 (32\%) participants in Chivite 2008. Publication bias was not assessable.

\section{Activities of daily living}

\section{Time point closest to 90 days}

Two trials with a total of 569 participants recorded activities of daily living scores (Chivite 2008; Strøm 2017a). Neither of the trials conducted intention-to-treat analysis for activities of daily living. In one trial, information was missing for 21 of 139 participants $(15 \%)$, for unknown reasons. In the other trial, information was missing for 89 of 430 participants $(21 \%)$; of these $54(13 \%)$ died before the 90-day follow-up (Strøm 2017a). Neither of the trials were published. Chivite 2008's authors stated that they had never sought to publish the trial. They used Older Americans Resources and Services instrumental Activities of Daily Living-score (OARS-
iADL; Pfeiffer 1978). Strøm 2017a was completed and the trial paper submitted for peer review. The trial authors used Lawton's Instrumental Activities of Daily Living Scale (Lawton's iADL; Lawton 1969).

The OARS-iADL Questionnaire consists of seven questions administered by a rater who solicits a self-report response from the participant, assessing different activities of daily living tasks; use of telephone, transportation, shopping, meal preparation, housework, medications, and money management. For each question, the participant responds by indicating whether the task can be performed independently, with some assistance, or not at all. Each domain is assessed on a 0 to 2 scale, giving a range of 0 to 14 (14 represents complete independence in all and 0 complete dependence).

The Lawton IADL score assesses eight independent living skills (ability to use telephone, shopping, food preparation, housekeeping, laundering, transportation, responsibility for own medications, and ability to handle finances. Participants are scored according to their highest level of functioning in that category (score of 0 or 1 per domain). A summary score ranges from 0 (low function, dependent) to 8 (high function, independent).

We were not able to pool the results for activities of daily living in a meaningful meta-analysis.

Chivite 2008 reported that the intervention group had a lower mean OARS-iADL score at 90 days (mean score 8 (SD 8), 59 of 70 randomised participants $(84 \%)$ ) than the usual-care group (mean score 9 (SD 3), 59 of 69 randomised participants (86\%); MD -1.00, $95 \% \mathrm{Cl}-3.18$ to 1.18$)$.

Strøm 2017a reported that the median Lawton iADL score was 8 (IQR 6 to 8 ) in the intervention group and 8 (IQR 5 to 8 ) in the usualcare group at day 90 from admission. The MD was $-0.36(95 \% \mathrm{Cl}$ -0.81 to 0.9 ). Six participants (3\%) in the intervention group and 35 participants (20\%) in the usual-care group experienced a reduction in iADL-score at 90 days from admission. Nineteen participants $(10 \%)$ in the intervention group and nine participants $(5 \%)$ in the usual-care group had increased iADL-scores.

Publication bias was not assessable. Using GRADE, we assessed the certainty of the evidence to be very low for activities of daily living. We considered this outcome at high risk of bias. The reason for the GRADE judgement is outlined in Appendix 4 and Summary of findings for the main comparison.

\section{Time point at maximum follow-up}

Chivite 2008 recorded iADL score at a longer-term follow-up. At 12-months, the intervention group had lower mean OARS-iADL score (MD $-1.00,95 \% \mathrm{Cl}-3.18$ to 1.18 (intervention group: mean score 7 (SD 3), 48/70 (69\%) randomised participants; usual-care group: mean score 8 (SD 4), 47/69 (68\%) randomised participants). Information was missing in 44 of 139 (32\%) participants for unknown reasons. Publication bias was not assessable.

\section{Hospital readmission}

\section{Time point closest to 90 days}

Nine trials, with a total of 2219 participants, recorded the proportion of participants who were readmitted to hospital after index hospitalisation at the time point closest to 90 days (Chivite 
2008; Decker 2008; Farkouh 1998; Miller 2010; Miller 2013; Roberts 1997; Ross 2007; Strøm 2017a; Than 2014). Five trials reported results based on the intention-to-treat population (Decker 2008; Miller 2010; Miller 2013; Roberts 1997; Ross 2007). In a total of 466 of 2219 participants (21\%), information regarding hospital readmission was missing or participants were lost to follow-up (Chivite 2008; Farkouh 1998; Strøm 2017a; Than 2014). When excluding Farkouh 1998, results were missing in 42 of 2219 participants (2\%). A total of 110 of 873 short-stay unit participants (13\%) were readmitted after index hospitalisation compared with 147 of 880 usual-care participants (17\%). Data including the specific assessment time points closest to 90 days in each trial are presented in Table 9.

We were able to pool eight of the nine trials in the meta-analysis; one trial did not report the outcome numbers (Farkouh 1998 reported revisits to emergency department for cardiac problems, but no information on revisits that led to hospital readmission). It is uncertain whether there is any difference in hospital readmission at the time point closest to 90 days for participants hospitalised in short-stay units compared to usual care (RR $0.80,95 \% \mathrm{Cl}$ 0.54 to 1.19 ; participants $=1753$; studies $=8 ; 1^{2}=57 \%$, very low-certainty evidence, random-effects meta-analysis; Analysis 3.1). The visual inspection of the forest plots and the tests for statistical heterogeneity $(12=57 \% ; P=0.02)$ indicated substantial heterogeneity. Investigation of the forest plots showed that one trial alone could have accounted for the statistical heterogeneity. When we removed Strøm 2017a, heterogeneity was reduced, both visually and statistically (RR $0.98,95 \% \mathrm{Cl} 0.73$ to 1.31 ; participants $=1351$; studies $=7 ; 12=9 \%$; Analysis 3.17). Publication bias was not assessable (fewer than 10 trials in the meta-analysis).

The best-worst and worst-best case meta-analyses showed that incomplete outcome data bias alone did not have potential to influence the results. Best-worst random-effects meta-analysis: RR $0.72\left(95 \% \mathrm{Cl} 0.48\right.$ to 1.09 ; participants $=1795$; studies $=8 ; \mathrm{I}^{2}=$ $64 \%$, very low-certainty evidence; Analysis 3.2), worst-best randomeffects meta-analysis: RR $0.93(95 \% \mathrm{Cl} 0.67$ to 1.29 ; participants = 1795 ; studies $=8 ; 1^{2}=45 \%$, very low-certainty evidence; Analysis 3.3). We imputed data for three trials (Chivite 2008; Strøm 2017a; Than 2014). We did not include Farkouh 1998 due to the lack of information. None of the additional analyses showed evidence of important influence of the investigated factors (trial publication status, time point of outcome assessment; Analysis 3.4; Analysis 3.5) or subgroup differences (Analysis 3.6; Analysis 3.7; Analysis 3.8).

Using GRADE, we assessed the certainty of the evidence to be very low for hospital readmission. We considered readmission to be an objective outcome. However, we downgraded the outcome one level for risk of bias due to all the trials being at high risk of bias in the GRADE assessment. The reason for the GRADE judgement is outlined in Appendix 4 and Summary of findings for the main comparison.

\section{Time point at maximum follow-up}

Nine trials, with a total of 2219 participants, obtained the proportion of participants who were readmitted to hospital after index hospitalisation at maximum follow-up (Decker 2008; Chivite 2008; Farkouh 1998; Miller 2010; Miller 2013; Roberts 1997; Ross 2007; Strøm 2017a; Than 2014). Four trials reported results based on the intention-to-treat population (Decker 2008; Miller 2013; Roberts 1997; Ross 2007). In a total of 488 of 2219 participants
(22\%), information regarding hospital readmission was missing or participants were lost to follow-up (Chivite 2008; Farkouh 1998; Miller 2010; Strøm 2017a; Than 2014). When we excluded Farkouh 1998, results were only missing in 64 of 2219 of participants (3\%). A total of 122 of 860 short-stay unit participants (14.2\%) were readmitted after index hospitalisation compared with 171 of 871 usual-care participants (20\%). Data including the specific assessment time points at maximum follow-up in each trial are presented in Table 10.

We were able to pool eight of the nine trials in the meta-analysis; one trial did not report the outcome numbers (Farkouh 1998 reported revisits to emergency department for cardiac problems, but no information on revisits that led to hospital readmission). It is uncertain whether there is any difference in hospital readmission at maximum follow-up for participants hospitalised in short-stay units compared to usual care (RR $0.75,95 \% \mathrm{Cl} 0.51$ to 1.10 ; participants $=1731$; studies $=8 ;\left.\right|^{2}=65 \%$, very low-certainty evidence, randomeffects meta-analysis; Analysis 3.9). Visual inspection of the forest plots and the test for statistical heterogeneity $\left(I^{2}=65 \% ; P=0.005\right)$ indicated significant heterogeneity. Contrary to the analysis at the time point of 90 days, no single trial could account for the statistical heterogeneity when investigating forest plots. When we removed Strøm 2017a, heterogeneity was only marginally reduced ( $\mathrm{RR} 0.85,95 \% \mathrm{Cl} 0.59$ to 1.22 ; participants $=1329$; studies $=7$; $12=48 \%$; Analysis 3.18), but when we also removed Miller 2010, heterogeneity was removed, both visually and statistically (RR 1.01, $95 \% \mathrm{Cl} 0.79$ to 1.30 ; participants $=1220$; studies $=6 ; 12=0 \%$; Analysis 3.19). Publication bias was not assessable (fewer than 10 trials in the meta-analysis).

The best-worst and worst-best case meta-analyses showed that incomplete outcome data bias alone had the potential to influence the results. Best-worst random-effects meta-analysis: RR 0.65 (95\% $\mathrm{Cl} 0.45$ to 0.94 ; participants $=1795$; studies $=8 ; \mathrm{I}^{2}=65 \%$, very low-certainty evidence; Analysis 3.10), worst-best random-effects meta-analysis: RR $0.88(95 \% \mathrm{Cl} 0.59$ to 1.33 ; participants = 1795 ; studies $=8 ; 1^{2}=73 \%$, very low-certainty evidence; Analysis 3.11). We imputed data for four trials (Chivite 2008; Miller 2010; Strøm 2017a; Than 2014). We did not include Farkouh 1998 due to the lack of information. None of the additional analyses showed evidence of important influence of the investigated factors (trial publication status, time point of outcome assessment; Analysis 3.12; Analysis 3.13) or subgroup differences (Analysis 3.14; Analysis 3.15; Analysis 3.16).

\section{Non-serious adverse events}

\section{Time point closest to 90 days and time point at maximum follow-up}

Two trials, with a total of 533 participants, recorded non-serious adverse events (Shen 2004; Strøm 2017a). We were not able to pool the results for non-serious adverse events in a meaningful metaanalysis.

Shen 2004 obtained intention-to-treat data for non-serious adverse events and found no non-serious adverse events in either the intervention group or the usual-care group (Shen 2004).

Strøm 2017a excluded 12 of 430 participants (3\%), due to inclusion errors or withdrawals and did not impute data for those participants (seven in intervention group compared with five in usual-care group). Sixteen participants (8\%) in the intervention 
group and 45 participants (21\%) in the usual-care group experienced at least one adverse event during hospitalisation (OR $0.31 ; 95 \% \mathrm{Cl} 0.17$ to $0.56 ; \mathrm{P}<0.001$ ). Trial authors did not distinguish between whether the events were non-serious or serious. There was one time point assessment.

Publication bias was not assessable. Using GRADE, we assessed the certainty of the evidence to be very low for non-serious adverse events. We considered this outcome at high risk of bias. The reason for the GRADE judgement is outlined in Appendix 4 and Summary of findings for the main comparison.

\section{Transfer to another department}

\section{Time point closest to 90 days and time point at maximum follow-up}

One trial with a total of 430 participants reported transfers to other departments after initial placement in a short-stay unit or a usual-care unit; $47(23 \%)$ in the intervention group compared with $31(15 \%)$ in the usual-care group were transferred to another treatment facility during hospitalisation (OR 1.69; 95\% CI 1.02;1.18, 418/430 participants analysed) (Strøm 2017a).

Seven trials with 1429 participants reported all admissions to inhospital wards (other than short-stay units) (McDermott 1997; Miller 2010; Roberts 1997; Ross 2007; Shen 2004; Sun 2014; Than 2014); however, as usual care usually consisted of admission to an in hospital ward, we found the comparison between short-stay unit hospitalisation and usual care unreasonable and we have just reported findings. Six trials reported admissions for the intentionto-treat population (McDermott 1997; Miller 2010; Roberts 1997; Ross 2007; Shen 2004; Sun 2014).

We grouped by condition the proportions (\%) of participants in the intervention group versus the usual-care group who were admitted to in-hospital wards other than short-stay units:

- asthma: $41 \%$ versus $100 \%$ (McDermott 1997)

- chest pain: $45.1 \%$ versus $100 \%$ (Miller 2010); $21 \%$ versus $95 \%$ (Roberts 1997); 13\% versus unclear (Than 2014)

- syncope: $43 \%$ versus $98 \%$ (Shen 2004); $15 \%$ versus 92\% (Sun 2014)

- transient ischaemic attack: $15 \%$ versus $100 \%$ (Ross 2007 ).

Publication bias was not assessable. Using GRADE, we assessed the certainty of the evidence to be very low for serious adverse events. We considered this outcome at high risk of bias. The reason for the GRADE judgement is outlined in Appendix 4 and Summary of findings for the main comparison.

\section{Length of stay in hospital}

We obtained data on length of stay in hospital from 12 trials, including 2224 participants (Chivite 2008; Decker 2008; Farkouh 1998; Gomez 1996; McDermott 1997; Miller 2010; Miller 2013; Roberts 1997; Ross 2007; Shen 2004; Strøm 2017a; Sun 2014). Seven trials conducted intention-to-treat analysis for total length of stay in hospital (Chivite 2008; Decker 2008; Gomez 1996; Miller 2010; Miller 2013; Roberts 1997; Ross 2007). Data were missing in at least 44 of 2226 participants (2.0\%) (McDermott 1997; Shen 2004; Strøm 2017a; Sun 2014). The number of participants that were evaluated and missing was not available in one trial that randomised 424 participants (Farkouh 1998).
We did not perform meta-analyses for numerous reasons. First of all, sample sizes of individual trials were rather small and we suspected that the distribution of values was skewed. It was inappropriate to use median values directly in a meta-analysis (Higgins 2011d, chapter 7.7.3.5). We were not able to collect appropriate data summaries or individual participant data from all trial authors in order to handle data on a transformed scale, such as a log scale, which potentially could reduce skew (Deeks 2011, chapter 9.4.5.3). Furthermore, two of the trials exclusively reported mean or median length of stay in hospital for selected subgroups (McDermott 1997; Shen 2004). One trial reported the mean length of stay in hospital exclusively for participants transferred to an inhospital ward; that is, any hospital ward other than short-stay unit, during their hospital stay ( $43 \%$ of the intervention group compared with $99 \%$ of the usual-care group; Shen 2004). The average length of stay in hospital was longest in the intervention group (70 hours in the intervention group compared with 65 hours in the usualcare group; Shen 2004). One trial reported the mean length of stay in hospital for the intervention group by two subgroups; that is, participants transferred to another in-hospital ward after treatment in the short-stay unit and participants discharged directly from the short-stay unit. They found very short average length of stay in hospital for the discharged short-stay unit-participants (mean 9 hours), but longer stays for the subgroup of the transferred shortstay unit participants (mean 77 hours) in comparison with the usual-care group (mean 59 hours; McDermott 1997). Finally, one trial did not report length of stay in hospital for the usual-care group (Farkouh 1998). The remaining trials reported shorter hospital stays in the intervention groups compared with the usual-care groups; mean or median length of stay in hospital ranged from 9 hours to 96 hours in the intervention groups and from 37 hours to 216 hours in the usual-care groups (Chivite 2008; Decker 2008; Gomez 1996; Miller 2010; Miller 2013; Roberts 1997; Ross 2007; Strøm 2017a; Sun 2014). In two trials, the reported mean or median length of stay in hospital in the intervention group was less than 24 hours (Decker 2008; Gomez 1996). In five trials, the reported mean or median length of stay in hospital in the intervention group was between 24 and 48 hours (Miller 2010; Miller 2013; Roberts 1997; Ross 2007; Sun 2014). In two trials, the reported mean or median length of stay in hospital in the intervention group was between 96 and 120 hours (Chivite 2008; Strøm 2017a); both trials included older participants (aged 65 or 75 years or older, respectively).

For each trial, details on length of stay in hospital measurements and data are presented in Table 11.

Publication bias was not assessable. Using GRADE, we assessed the certainty of the evidence to be very low for length of stay. We considered length of stay to be an objective outcome at low risk of bias. However, we downgraded the outcome one level for risk of bias due to all the trials being at high risk of bias in the GRADE assessment. The reason for the GRADE judgement is outlined in Appendix 4.

\section{Comparable costs}

Eighttrials, including 1433 participants, reported costs (Chivite 2008; Farkouh 1998; Gomez 1996; McDermott 1997; Miller 2010; Roberts 1997; Ross 2007; Sun 2014). Four trials conducted intention-to-treat analysis for costs (Gomez 1996; Miller 2010; Miller 2013; Ross 2007; Sun 2014). One trial exclusively analysed the first 130 of 222 randomised participants (59\%) (McDermott 1997). Two trials did not clearly define the number of included participants in 
the cost analyses (Chivite 2008; Farkouh 1998). In the remaining trial, information was missing for one participant (0.01\%) (Roberts 1997).

As suspected prior to conducting the review, there was substantial heterogeneity between the assessment of costs in the trials; that is, two trials used the exact same method to calculate costs, and the variability across settings was high; for example, trials used different treatment protocols, there were differences in local pricing and inter-country variability in cost-estimations. All trials indicated that the short-stay unit model had cost-saving properties compared with usual care. Cost measurements are summarised in Table 12. In the following narrative summary of data, we have presented the findings by condition.

\section{Asthma}

McDermott 1997 assessed hospital costs for the index hospital stay by a full evaluation of used health care resources during the index hospital stay (e.g. including individual tests and resources used per participant, fixed and variable costs for running the department and hospital, labour expenses). Short-stay unit hospitalisation rather than usual care (inpatient treatment) incurred lower costs for participants with acute exacerbation of asthma. Mean costs (SD) per participant in intervention group compared with usual-care group were USD 1202 (SD USD 1343) compared with USD 2247 (SD USD 1110).

\section{Chest pain}

Roberts 1997 assessed hospital costs for the index hospital stay using similar methods to McDermott 1997, in participants with chest pain and low probability of acute myocardial infarction. Mean hospital costs were lower in the intervention group; mean hospital costs per participant (SD) in intervention group compared with usual-care group were USD 1528 (SD USD 1012) compared with USD 2095 (SD USD 2095).

Farkouh 1998 assessed six-month costs related to cardiovascular care in participants with chest pain who were stratified to be at intermediate risk for cardiovascular events in the short term. Costs were derived upon relative-value units for cardiac tests, cardiac procedures, and cardiac hospitalisation. Each unit was given a relative weight, and the weighted frequencies were added together for a summary score for each participant. Trial authors did not present the costs per group, but reported that a participant in the usual-care group would incur, on average, approximately $61 \%$ more costs related to cardiac care during a period of six months in comparison with a participant in the intervention group.

Gomez 1996 assessed hospital costs for the index hospital stay and for a follow-up period of 30 days using hospital charges in participants with low-risk chest pain. Calculation of costs was based on itemised hospital accounts of the enrolled participants. Participants in the intervention group had lower index hospital stay charges and 30-day hospital charges as compared with the usualcare group. For the index hospital charges, median charges (IQR) in intervention group compared with usual-care group were USD 895 (USD 712 to USD 991) compared with USD 1488 (USD 1096 to USD 3546). For the 30-day total hospital charges, median charges in intervention group compared with usual-care group (IQR) were USD 904 (USD 731 to USD 1347) compared with USD 1542 (USD 1142 to USD 3845).
Miller 2010 assessed hospital costs for the index hospital stay using hospital charges in participants with intermediate to high-risk chest pain. Trial authors compared a diagnostic protocol including upfront cardiac stress-magnetic resonance imaging (MRI) in a shortstay unit (intervention group) with standard inpatient care (usualcare group). Costs were derived as the sum of hospital and provider costs calculated from itemised hospital charges. The estimated mean difference in direct costs was USD 588 (95\% CI USD 336 to USD 811) in favour of the intervention group (median direct costs (IQR) in the intervention group compared with the usual-care group were USD 2062 (USD 1918 to USD 2367) compared with USD 2680 (USD 2408 to USD 3448)). In a one-year follow-up study, trial authors used billing information for cardiac-related healthcare encounters and found that cardiac costs from enrolment through 360 days were lower for the intervention group (median costs (IQR) in short-stay unit-group compared with usual-care group: USD 2186 (USD 1957 to USD 4308) compared with USD 3850 (USD 2669 to USD 9710); Miller 2011).

\section{Heart failure}

Chivite 2008 assessed hospital costs for the index hospital stay and total hospital costs during a not-clearly-defined follow-up period in older participants (aged 65 years or older) with acute decompensated heart failure. Trial authors found reduced hospital costs for the index admission in the intervention group; mean total hospital costs (SD) in the intervention group compared with the usual-care group were EUR 779.43 (EUR 573.09) compared with EUR 2311.12 (EUR 1847.46). For total costs in the follow-up period including days of hospital stay, tests, new admissions or emergency department attendance costs including tests, transfers (ambulance costs), mean total costs (SD) were EUR 2488.60 (EUR 956.62) in the intervention group compared with EUR 3574.14 (EUR 1018.95) in the usual-care group (findings obtained after contact with trial authors).

\section{Syncope}

Sun 2014 assessed hospital costs for the index hospital stay and total hospital costs within 30 days by imputing US national Medicare mean payments for procedures and observation facility fees to estimate the hospital facility cost per unit of time. The total facility cost for each participant was estimated based on the hospital length-of-stay (time of emergency department-arrival to time of hospital-discharge), and costs of the procedures that were performed were added. An absolute cost reduction of USD 629 was found in the intervention group (95\% CI USD -1376 to USD -56). Median index visit hospital costs (IQR) for intervention group compared with usual-care group were USD 1210 (USD 948 to USD 1660) compared with USD 1580 (USD 870 to USD 2390). For hospital costs within 30 days, the difference was lower; that is, USD 479 ( $95 \%$ CI USD -1230 to USD 198).

\section{Transient ischaemic attack}

Ross 2007 assessed hospital costs for the index hospital stay and 90-day total costs in participants with transient ischaemic attack. Both median index visit costs and median 90-day total costs were lower in the intervention group. Index hospital stay cost calculations included individual resource use, facility costs, and overhead expenses of running the hospital, but did not include staff costs (physicians, nurses). The 90-day costs were calculated by adding costs related to return visits to the index hospital stay costs. Median index hospital stay costs (IQR) for the intervention group 
compared with the usual-care group were USD 864 (USD 726 to USD 1076) compared with USD 1529 (USD 1091 to USD 2306). Median 90day total hospital costs (IQR) for the intervention group compared with the usual-care group were USD 890 (USD 768 to USD 1510) compared with USD 1548 (USD 1091 to USD 2474).

Publication bias was not assessable. Using GRADE, we assessed the certainty of the evidence to be very low for costs. We considered this outcome at high risk of bias. The reason for the GRADE judgement is outlined in Appendix 4 and Summary of findings for the main comparison

\section{Missing data}

We have reported details on missing data for each outcome. Few trials conducted intention-to-treat analysis in all analyses, but overall, trials had very little missing information due to low exclusion or lost-to-follow-up rates.

\section{Unit of analysis issues}

We did not identify unit of analysis issues among the included trials.

\section{Assessments of the certainty of the body of evidence}

We assessed the certainty of the evidence using the GRADE approach for the results of the most important outcomes at the time point closest to 90 days. GRADE assessments showed that the certainty of the evidence must be regarded as very low despite the body of evidence being from randomised trials. We reduced the certainty ratings because of high likelihood of bias in the included trials, substantial clinical heterogeneity among populations and co-interventions, substantial statistical heterogeneity, indirectness of evidence, or imprecision in the effect estimates (for GRADE assessment details see Summary of findings for the main comparison and Appendix 4).

\section{DISCUSSION}

\section{Summary of main results}

We included 14 completed trials randomising 2872 adults to either short-stay unit hospitalisation or usual care for internal medicine diseases or conditions. We identified one ongoing trial that was commenced one week prior to the search. Only completed trials were included in our analyses. All trials were at high risk of bias. We assessed the evidence for all outcomes to be of very low certainty. The components of the intervention, shortstay unit hospitalisation, and the participant populations were heterogeneous. We found limited data for all outcomes.

Our analyses showed that it is uncertain whether hospitalisation in short-stay units compared with usual care affects mortality, serious adverse events, or hospital readmission. There was not enough information to confirm or refute that short-stay unit hospitalisation had relevant effects on quality of life, activities of daily living, non-serious adverse events, and costs. We were merely able to compile results for these outcomes in a narrative way, because either the trial authors had used different outcome scoring systems, data were too sparse, or they were presented in an unusable way. Overall, the results of the individual trials indicated time and cost-sparing effects of short-stay unit hospitalisation, and higher quality-of-life scores for participants randomised to short-stay unit hospitalisation. However, it is crucial to validate the findings in larger, well-conducted trials, and one should abstain from spurious interpretations.

Our main results are summarised in Summary of findings for the main comparison.

\section{Overall completeness and applicability of evidence}

We were aware that earlier reviews of the literature found problems in identifying trials on short-stay units due to terminology issues. We therefore conducted a broad literature search in close collaboration with a Cochrane Information Specialist (PM). Compared with previous systematic reviews, we identified far more trials (in total 15 trials compared with five to seven trials (Daly 2003; Damiani 2011; Downing 2008; Galipeau 2015). We searched for published and unpublished trials, irrespective of publication type, publication date, publication status, and language. Additionally, we searched the grey literature, and bibliographies of all included trials and earlier systematic reviews to identify missing trials.

The included trials were clinically heterogeneous; there was a large span in participant selection criteria and components of the intervention, short-stay unit hospitalisation. Mostly, the intervention comprised short-stay unit hospitalisation including application of well-defined clinical protocols. The components of the clinical protocols were mainly dictated by the conditions studied in the individual trials. All but one trial clearly described a target time frame for stay in the short-stay unit below 120 hours (five days) with a majority of trials aiming at stays under 24 hours (11 of 14 trials).

We were not able to create funnel plots for detection of publication bias due to limited available data. We identified two unpublished trials; contact with trial authors revealed that one was completed and submitted for peer review (Strøm 2017a) and publication was never sought for the other (Chivite 2008).

We conducted subgroup analyses to explore potential evidence of differences in patient outcomes related to the subtypes of shortstay units (multipurpose units compared with units dedicated to a single condition or disease, units applying protocol-specific cointerventions in short-stay unit compared with no protocol-specific co-interventions) or participant-specific characteristics (younger compared with older participants). None of the subgroup analyses showed evidence of such effects. We observed that missing data had the potential to influence the results of the analyses on mortality, hospital readmission, and serious adverse events; tests of best-worst case scenarios showed a potential effect of shortstay unit hospitalisation on mortality (at the time point closest to 90 days and at maximum follow-up) and hospital readmission (at maximum follow-up), while tests for worst-best case scenarios showed potentially higher risk of adverse events in short-stay unit participants (at maximum follow-up).

We used two time points for outcome assessments: at the time point closest to 90 days and at maximum follow-up. It is always difficult to choose the optimal assessment time point. The observation period needs to be long enough, so that the participants experience events; on the other hand, if the observation period is too long, events not related to the disease or the intervention might dilute the actual trial intervention effects and reduce power. We chose the time point closest to 90 days to be the primary time point of interest, because we anticipated that 
many trial participants were likely to have several admissions that could interfere with the intervention effect. Hence, a short-term effect of the intervention would be most reliable.

Our analyses showed that at this point in time, there is not enough information to confirm or refute that short-stay unit hospitalisation has beneficial or harmful effects on mortality, serious adverse events, quality of life, activities of daily living, hospital readmissions, non-serious adverse events, transfer to another department, length of stay in hospital, and costs.

\section{Certainty of the evidence}

We assessed the certainty of the evidence for all outcomes (Summary of findings for the main comparison). Despite exclusively including randomised trials, the GRADE assessments showed that the certainty of the evidence was very low for all of the outcomes. Reasons for the GRADE assessments are provided in the footnotes of the Summary of findings for the main comparison.

\section{Potential biases in the review process}

\section{Strength and limitations of the review}

\section{Strengths}

This review was conducted in compliance with Cochrane recommendations (Higgins 2011a). We published the review protocol prior to the first literature search to ensure transparency (Strøm 2016). We conducted the review according to the published protocol (Strøm 2016) and reported all deviations (see Differences between protocol and review). We limited publication bias by conducting an extensive literature search that included databases of both indexed and non-indexed trial records, and sources of unpublished literature. Two individual review authors screened trials for inclusion in order to reduce the risk of missing an eligible trial, and two independent review authors extracted all trial data and evaluated risk of bias domains to validate data and enhance the external validity of this review.

We exclusively included randomised trials to ensure scientific rigour, because observational studies cannot reliably evaluate intervention effects (Savović 2012) and non-randomised trials are prone to selection bias and confounding (Higgins 2011a). We conducted a comprehensive search of the literature and included trials regardless of publication type, publication status, choice of outcomes, and language. We were aware that earlier reviews had problems in identifying trials on short-stay units due to terminology issues, and conducted a broad search that revealed far more trials than previous systematic reviews, which could not be explained by inclusion of new publications. Additionally, we searched the grey literature, and bibliographies of all included trials and earlier systematic reviews to identify missing trials.

We contacted all authors of the included trials to receive additional information and the response rate was high. We evaluated the risk of systematic error by thorough preplanned bias assessments. We explored the reasons behind substantial statistical heterogeneity, tested the robustness of our results with sensitivity analyses (bestworst case scenarios, etc.), and used subgroup analyses to assess the potential impact of bias on the results (Sterne 2011b; Jakobsen 2014).

\section{Limitations}

This review also had limitations. The included trials had several important methodological issues that warrant careful interpretation of the analyses. We classified all trials as high risk of bias; that is, the included trials might have been influenced by systematic errors. Trials with high risk of bias tend to overestimate benefits and underestimate harms (Savović 2012). The sample sizes of most trials were relatively small with four trials randomising more than 166 participants (Farkouh 1998; McDermott 1997; Strøm 2017a; Than 2014).

Despite the body of evidence for each outcome being based on randomised trial designs, our GRADE assessments showed that the certainty of the evidence must be regarded as very low. We exclusively identified trials investigating emergency departmentbased short-stay units; findings may not apply to other short-stay unit settings such as Acute Medical Wards. The trials' participant selection criteria and interventions were dictated by specific conditions and varied among the trials; clinical heterogeneity was present and may threaten the external validity of the findings. We described staffing details in Characteristics of included studies if available, but only two trials reported this (Chivite 2008; Strøm 2017a). The effect of short-stay unit hospitalisation may be confounded by concurrent provision of standardised observation or treatment protocols. We were not able to assess the risk of publication bias due to limited available data.

The lack of proper assessments of serious adverse events in individual trials translates into a flaw in this review. None of the trials reported serious adverse events according to the ICH-GCP guidelines (ICH-GCP 1997). Instead of reporting serious adverse events as proposed in our protocol (Strøm 2016), we used trial authors' definitions of events that we believed were important surrogate markers for serious adverse events. Such surrogate outcome is obviously at risk of reporting components with different degrees of severity.

We assessed outcomes at two time points; time point closest to 90 days to assess short-term effects, and at maximum follow-up to assess both short-term and long-term effects, but very few trials conducted a long-term follow-up.

We did not include an evaluation of emergency department service utilisation, or use of home care after hospitalisation, which could have enhanced the description of downstream effects of the intervention. However, we did not encounter such information in any of the included trials.

Three of the review authors (CS, LSR, TS) are involved in one of the included trials (Strøm 2017a), hence they may be subject to having a potential academic bias. However, we ensured that judgements regarding the given trial did not involve these review authors.

Finally, we did not use Trial Sequential Analysis or any other sequential analysis to assess the risk of random errors (Jakobsen 2014). Hence, we are unaware of the role of 'play of chance' in this present review.

\section{Agreements and disagreements with other studies or reviews}

Only a few systematic reviews have investigated effects of shortstay unit hospitalisation. In 2003, Daly and colleagues concluded 
that short-stay units had the potential to improve emergency department effectiveness, reduce length of hospital stays, and reduce costs (Daly 2003). A recent review specifically addressing effectiveness of acute medical units (i.e. short-stay units based in internal medicine departments) found a trend towards lower mortality and reduced average length of stay in hospital in participants treated in a short-stay unit-setup (Reid 2016). However, only observational studies were available and authors did not assess the certainty of evidence. Another recent review assessed the effectiveness and safety of emergency department-based short-stay units in comparison with usual care including both bias assessments and grading of the certainty of evidence (Galipeau 2015). They included five trials (Decker 2008; McDermott 1997; Miller 2010; Miller 2013; Roberts 1997), all of which were included in this present review. Authors emphasised that the terminology used to name short-stay units was variable and possessed a major challenge in identifying trials and completing the review. We identified a much larger number of trials, all of them emergency department-based and primarily multipurpose units. Compared with their bias assessment, we judged the risk of bias of the trials to be more profound (Galipeau 2015).

In large epidemiological studies of general internal medicine patients, mortality has been estimated to be around $10 \%$ within the first 48 hours (Marco 2010). In contrast, we observed that the mortality was very low across the included trials, indicating that the studied participants were at low risk of dying a priori. Such a low event rate necessitates a very large sample size to ascertain that short-stay unit hospitalisation is non-inferior to usual care. We found the highest mortality in trials including older participants, but advanced age did not seem to be associated with either increased or decreased effect of short-stay unit hospitalisation. Our mortality rates underline that the trial participants are a highly selected group, and it is uncertain to what extent short-stay unit hospitalisation is applicable to a general internal medicine population. How patients should be selected is still an area that should be explored. It is not known whether strict or pragmatic inclusion criteria is to be preferred. We explored whether multipurpose units compared with specialised units and protocol-based care compared with non-protocol-based care could have had an effect on our results in the meta-analysis. We did not find any evidence of such an effect, but data were sparse and the evidence of very low certainty, and this area should be investigated further in future trials. It is important to acknowledge that this short-stay unit feature should not be over-simplified, because local needs and settings may differ widely and such factors should be taken into account when organising short-stay unit care.

Trials and observational studies comparing the total number of participants treated in a short-stay unit compared with usual care indicate that short-stay unit hospitalisation is associated with significantly shorter hospital stays (Arendts 2006; Chivite 2008; Decker 2008; Farkouh 1998; Gomez 1996; Miller 2010; Miller 2013; Roberts 1997; Ross 2007; Salazar 2006; Strøm 2017a; Sun 2014). However, a comparison with other care models should be interpreted with caution, because short length of stay in hospital is an implicit part of the intervention. It may be reasonable to regard length of stay in hospital as a key performance indicator rather than an outcome.

The short-stay unit model seemed to have cost-sparing effects, but application of individual trial findings to other settings is limited due to the extensive differences in short-stay unit setup, finance, billing, and reimbursement between departments, hospitals, and countries. Eight out of nine trials assessing costs were conducted in the USA. US hospitals may have an interest in establishing shortstay units for economic reasons. A significant number of admissions to inpatient services are deemed improper by the Federal Medicare programme (Feng 2012). In such cases, hospitals have to refund the received Medicare payments. However, a loophole may be found in short-stay units. Physicians or hospitals may substitute an in-hospital admission with placement in a short-stay unit under so-called observation status for economic reasons (Feng 2012). We did not find any indication that such an economic incentive was present in the included trials; but hypothetically, the economic aspect could bias recommendations behind short-stay unit implementation.

\section{AUTHORS' CONCLUSIONS}

\section{Implications for practice}

In view of the quality of the evidence available, we are not able to determine the effects and compose evidence-based recommendations for or against short-stay unit hospitalisation in for adults with internal medicine diseases and conditions.

All trials were designed to measure the effect of emergency department-based short-stay unit hospitalisation in selected groups of patients. The rates of mortality and serious adverse events observed in the included trials were low, and it is uncertain to what extent short-stay unit hospitalisation affects these outcomes. It is also uncertain whether shortstay unit hospitalisation affects hospital readmissions. There was insufficient evidence to confirm or refute that short-stay unit hospitalisation had important effects on quality of life, activities of daily living, non-serious adverse events, transfer to another department, length of stay in hospital, and costs. The individual trials pointed in the direction of time- and cost-sparing effects and higher quality-of-life scores related to short-stay unit hospitalisation. However, these findings are not validated and warrant validation in larger and scientifically well-conducted trials. All of the trials were at high risk of bias, which is known to be associated with an inherent risk of overestimation of benefits and underestimation of harms.

\section{Implications for research}

Large and well-conducted randomised trials are needed that both assess beneficial and harmful effects of short-stay units. Previous trials have primarily estimated outcomes for a narrow subset of participants; there is a need to explore the general effectiveness of short-stay units. To enhance capture of available data, we encourage authors of future papers to use the term 'short-stay unit' to describe units that provide short-term or accelerated care for selected participants in hospital units with a target time frame of a maximum stay in the unit of five days. Furthermore, we encourage authors to report the specific target time frame, describe resources in detail (such as available equipment, staffing, including differences between intervention and comparison resources), and include evaluation of both patient-centred outcomes and health care utility, including cost-analysis. 
To date, trials have solely been conducted in emergency department-based settings. The benefits and drawbacks may be different in other settings and should be investigated.

\section{ACKN OWLEDGEMENTS}

We acknowledge the help and support of Cochrane Effective Practice and Organisation of Care, especially Paul Miller (PM) for tailoring the search strategy, and Managing Editor, Julia Worswick (JW). The review authors would also like to thank the following editors and peer referees, Gillian Leng (GL), Monica Taljaard (MT),
Saul Blecker (SB), Juliana Esther Martin-Lopez (EM), Peter D Griffiths (PG), Villyen Ngenkafac Motaze (VNM), Jonathan M. Fuchs (JMF), Jemma Hudson (JH), Toby Lasserson (TL), and Liz Bickerdike (LB).

National Institute for Health Research (NIHR), via Cochrane Infrastructure funding to Cochrane Effective Practice and Organisation of Care (EPOC). The views and opinions expressed herein are those of the review authors and do not necessarily reflect those of the Systematic Reviews Programme, NIHR, National Health Service (NHS) or the Department of Health. 


\section{R E F E R E N C E S}

\section{References to studies included in this review}

Chivite 2008 \{published data only\}

Chivite D, Salazar A, Pujol R, Alonso J. Short-stay units as an alternative to internal medicine for elderly patients with decompensated heart failure. European Journal of Internal Medicine 2008; Vol. 19:S40-1.

\section{Decker 2008 \{published data only\}}

Decker WW, Smars PA, Vaidyanathan L, Goyal DG, Boie ET, Stead LG, et al. A prospective, randomized trial of an emergency department observation unit for acute onset atrial fibrillation. Annals of Emergency Medicine 2008;52(4):322-8.

Farkouh 1998 \{published data only\} Farkouh ME, Smars PA, Reeder GS, Zinsmeister AR, Evans RW, Meloy TD, et al. A clinical trial of a chest-pain observation unit for patients with unstable angina. Chest Pain Evaluation in the Emergency Room (CHEER) Investigators. New England Journal of Medicine 1998;339(26):1882-8.

\section{Gomez 1996 \{published data only\}}

Gomez MA, Anderson JL, Karagounis LA, Muhlestein JB, Mooers FB. An emergency department-based protocol for rapidly ruling out myocardial ischemia reduces hospital time and expense: results of a randomized study (ROMIO). Journal of the American College of Cardiology 1996;28(1):25-33.

\section{McDermott 1997 \{published data only\}}

McDermott MF, Murphy DG, Zalenski RJ, Rydman RJ, McCarren M, Marder D, et al. A comparison between emergency diagnostic and treatment unit and inpatient care in the management of acute asthma. Archives of Internal Medicine 1997;157(1):2055-62.

\section{Miller 2010 \{published data only\}}

Miller CD, Hwang W, Hoekstra JW, Case D, Lefebvre C, Blumstein $\mathrm{H}$, et al. Stress cardiac magnetic resonance imaging with observation unit care reduces cost for patients with emergent chest pain: a randomized trial. Annals of Emergency Medicine 2010;56:209-19.

\section{Miller 2013 \{published data only\}}

Miller CD, Case LD, Little WC, Mahler SA, Burke GL, Harper EN, et al. Stress cardiac magnetic resonance imaging reduces revascularization, hospital readmission, and recurrent cardiac testing in intermediate risk patients with acute chest pain: a randomized trial. Journal of the American College of Cardiology Cardiovascular Imaging 2013;6:785-94.

\section{Roberts 1997 \{published data only\}}

Roberts RR, Zalenski RJ, Mensah EK, Rydman RJ, Ciavarella G, Gussow L, et al. Costs of an emergency department-based accelerated diagnostic protocol vs hospitalization in patients with chest pain: a randomized controlled trial. Journal of the American Medical Association 1997;26:1670-6.
Ross 2007 \{published data only\}

Ross MA, Compton S, Medado P, Fitzgerald M, Kilanowski P, O'Neil BJ. An emergency department diagnostic protocol for patients with transient ischemic attack: a randomized controlled trial. Annals of Emergency Medicine 2007;50(2):109-19.

\section{Rydman 1997 \{published data only\}}

Rydman RJ, Zalenski RJ, Roberts RR, Albrecht GA, Misiewicz VM, Kampe LM, et al. Patient satisfaction with an emergency department chest pain observation unit. Annals of Emergency Medicine 1997; Vol. 29, issue 1:109-15.

\section{Shen 2004 \{published data only\}}

Shen WK, Decker WW, Smars PA, Goyal DG, Walker AE, Hodge DO, et al. Syncope evaluation in the emergency department study: a multidisciplinary approach to syncope management. Circulation 2004; Vol. 110, issue 24:3636-45.

\section{Strøm 2017a \{unpublished data only\}}

Strøm C, Rasmussen LS, Löwe AS, Lorentzen AK, Lohse N, Benn Madsen KH, et al. Short-stay unit hospitalisation in older internal medicine patients - a randomised trial. Clinicaltrials.gov (accepted for publication in Age Ageing 2 May 2018). [NCT02395718]

\section{Sun 2014 \{published data only\}}

Sun BC, McCreath H, Liang L, Bohan S, Baugh C, Ragsdale L, et al. Randomised clinical trial of an emergency department observation syncope protocol vs. routine inpatient admission. Annals of Emergency Medicine 2014;64(2):167-75.

Than 2014 \{published data only\}

Than M, Aldous S, Lord SJ, Goodacre S, Frampton CM, Troughton R, et al. A 2-hour diagnostic protocol for possible cardiac chest pain in the emergency department: a randomized clinical trial. Journal of the American Medical Association Internal Medicine 2014;174(1):51-8.

\section{References to studies excluded from this review}

Abdelaziz 2016 \{published data only\}

Abdelaziz H, Al Anany R, Elmalik A, Saad M, Prabhu K, AlTamimi $\mathrm{H}$, et al. Impact of clinical pharmacy services in a short stay unit of a hospital emergency department in Qatar. International Journal of Clinical Pharmacy 2016;38(4):776-9.

\section{Aggarwal 1995 \{published data only\}}

Aggarwal P, Wali J P, Ranganathan S, Kailash S, Kumar A, Mishra MC. Utility of an observation unit in the emergency department of a tertiary care hospital in India. European Journal of Emergency Medicine 1995;2(1):1-5.

\section{Aitken 1993 \{published data only\}}

Aitken PD, Rodgers H, French JM, Bates D, James OF. General medical or geriatric unit care for acute stroke? A controlled trial. Age and ageing 1993;22:4-5. 
Alonso 2010 \{published data only\}

Alonso G, Escudero JM. The emergency department short stay unit and the hospital at home as alternatives to standard inpatient hospitalisation. Anales Del Sistema Sanitario De Navarra 2010;33:97-106.

\section{Applegate 1990 \{published data only\}}

Applegate WB, Miller ST, Graney MJ, Elam JT, Burns R, Akins DE. A randomized, controlled trial of a geriatric assessment unit in a community rehabilitation hospital. American Journal of Public Health 1990;322(22):1572-8.

\section{Applegate 1991 \{published data only\}}

Applegate WB, Graney MJ, Miller ST, Elam JT. Impact of a geriatric assessment unit on subsequent health care charges. New England Journal of Medicine 1991;81(10):1302-6.

\section{Arendts 2006 \{published data only\}}

Arendts G, MacKenzie J, Lee JK. Discharge planning and patient satisfaction in an emergency short-stay unit. Emergency Medicine Australasia 2006;18(1):7-14.

\section{Asplund 2000 \{published data only\}}

Asplund K, Gustafson Y, Jacobsson C, Bucht G, Wahlin A, Peterson J, et al. Geriatric-based versus general wards for older acute medical patients: a randomized comparison of outcomes and use of resources. Journal of the American Geriatrics Society 2000;48(11):1381-8.

\section{Barbado 1999 \{published data only\}}

Barbado AM, Jimeno Carruez A, Ostolaza Vázquez JM, Molinero de Dios J. Short-stay units depending on internal medicine. Anales de Medicina Interna 1999;16(10):504-10.

\section{Barberger-Gateau 1986 \{published data only\}}

Barberger-Gateau P, Dabis F, Moise A, Gimbert M, Galley P, Salamon R. Factors related to length of hospitalization of elderly patients during short stay. Revue d'Epidemiologie et de Sante Publique 1986;35(6):463-73.

\section{Barberger-Gateau 1989 \{published data only\}}

Barberger-Gateau P, Grolier L, Maurice S, Borde C, Salamon R, Galley P. Short-stay hospitalization of elderly persons: first step toward institutionalization?. Revue d'Epidemiologie et de Sante Publique 1989;38(4):323-32.

\section{Barnes 2012 \{published data only\}}

Barnes DE, Palmer RM, Kresevic DM, Fortinsky RH, Kowal J, Chren MM, et al. Acute care for elders units produced shorter hospital stays at lower cost while maintaining patients' functional status. Health Affairs (Project Hope) 2012;31(6):1227-36.

\section{Basic 2009 \{published data only\}}

Basic D, Khoo AK. Admission variables predicting short lengths of stay of acutely unwell older patients: relevance to emergency and medical short-stay units. Australian Health Review 2009;33(3):502-12.

\section{Baugh 2011 \{published data only\}}

Baugh CW, Venkatesh AK, Stephen BJ. Emergency department observation units: a clinical and financial benefit for hospitals. Health Care Management Review 2011;36(1):28-37.

\section{Baugh 2012 \{published data only\}}

Baugh CW, Venkatesh AK, Hilton JA, Samuel P A, Schuur JD, Stephen BJ. Making greater use of dedicated hospital observation units for many short-stay patients could save $\$ 3.1$ billion a year. Health Affairs 2012;31(10):2314-23.

\section{Baugh 2014 \{published data only\}}

Baugh CW, Stephen BJ. Emergency department observation units. In: Stephanie Kayden, Philip D Anderson, Robert Freitas, Elke Platz editor(s). Emergency Department Leadership and Management: Best Principles and Practice. 1st Edition. Cambridge University Press, 2014:234. [ISBN-13: 978-1107007390]

\section{Blecker 2014 \{published data only\}}

Blecker S, Ladapo JA, Doran KM, Goldfeld KS, Katz S. Emergency department visits for heart failure and subsequent hospitalization or observation unit admission. American Heart Journal 2014;168(6):901-8.e1.

\section{Bogaty 2001 \{published data only\}}

Bogaty P, Dumont S, O'Hara GE, Boyer L, Auclair L, Jobin J, et al. Randomized trial of a noninvasive strategy to reduce hospital stay for patients with low-risk myocardial infarction. Journal of the American College of Cardiology 2001;37(5):1289-96.

\section{Broquetas 2008 \{published data only\}}

Broquetas JM, Pedreny R, Martínez-Llorens JM, Sellarésa J, Gea J. Short-stay respiratory unit: a new option for inpatient care. Archivos de Bronconeumología 2008;44(5):252-6.

\section{Bullard 2007 \{unpublished data only\}}

Bullard M. Clinical Decision Unit (CDU) - evaluation of a novel approach to address emergency department overcrowding. Clinicaltrials.gov. [NCT00497393]

Burkhardt 2005 \{published data only\}

Burkhardt J, Peacock WF, Emerman CL. Predictors of emergency department observation unit outcomes. Academic Emergency Medicine 2005;12(9):869-74.

\section{Carpentier 2001 \{published data only\}}

Carpentier F, Guignier M, Lambert-Eytan V. Emergency department observation units. Therapie 2001;56(2):151-5.

\section{Caterino 2013 \{published data only\}}

Caterino JM, Hoover EM, Moseley MG. Effect of advanced age and vital signs on admission from an emergency department observation unit. American Journal of Emergency Medicine 2013;31(1):1-7.

\section{Chen 2005 \{published data only\}}

Chen Cl, Liu CY, Li YC, Chao CC, Liu CT, Chen CF, et al. Pervasive observation medicine: the application of RFID to improve patient safety in observation unit of hospital emergency 
department. Studies in Health Technology and Informatics 2005;116:311-5.

\section{Cheng 2016 \{published data only\}}

Cheng AH, Barclay NG, Abu-Laban RB. Effect of a multidiagnosis observation unit on emergency department length of stay and inpatient admission rate at two Canadian hospitals. The Journal of Emergency Medicine 2016;51(6):739-47.

\section{Choi 1999 \{published data only\}}

Choi SP, Park KN, Park SH, Kim SK, Kim YM, Lee WJ, et al. Utilization of the short-stay unit in emergency department. Journal of the Korean Society of Emergency Medicine 1999;10(2):183-90.

\section{Claesson 2000 \{published data only\}}

Claesson L, Gosman-Hedstrom G, Johannesson M, Fagerberg B, Blomstrand C. Resource utilization and costs of stroke unit care integrated in a care continuum: a 1-year controlled, prospective, randomized study in elderly patients: the Goteborg $70+$ Stroke Study. European Journal of Neurology 2000;31(11):2569-77.

\section{Claesson 2003 \{published data only\}}

Claesson L, Gosman-Hedstrom G, Fagerberg B, Blomstrand C. Hospital re-admissions in relation to acute stroke unit care versus conventional care in elderly patients the first year after stroke: the Goteborg 70+ Stroke study. Age and Ageing 2003;32(1):109-13.

\section{Clemson 2016 \{published data only\}}

Clemson L, Lannin NA, Wales K, Salkeld G, Rubenstein L, Gitlin L, et al. Occupational therapy predischarge home visits in acute hospital care: a randomized trial. Journal of the American Geriatrics Society 2016;10:2019-26.

\section{Cochard 1999 \{published data only\}}

Cochard R. Implementing a rapid treatment and short-stay unit. SSM (abbreviation unclear). 1999; Vol. 5, issue 11:53.

\section{Collier 2007 \{published data only\}}

Bernhardt J, Dewey H, Thrift A, Collier J, Donnan G. A very early rehabilitation trial for stroke (AVERT): phase II safety and feasibility. Stroke 2008;39(2):390-6.

\section{Collins 2013 \{published data only\}}

Collins SP, Pang PS, Fonarow GC, Yancy CW, Bonow RO, Gheorghiade M. Is hospital admission for heart failure really necessary? The role of the emergency department and observation unit in preventing hospitalization and rehospitalization. Journal of the American College of Cardiology 2013;61(2):121-6.

\section{Corbella 2002 \{published data only\}}

Corbella X, Salazar A, Maiques JM, Juan A. Emergency department short-stay unit as an alternative to standard inpatient care hospitalization. Medicina Clínica Facultad de Medicina de Barcelona 2002;118(13):515-6.

\section{Covinsky 1997 \{published data only\}}

Covinsky KE, King JT Jr, Quinn LM, Siddique R, Palmer R, Kresevic DM, et al. Do acute care for elders units increase hospital costs? A cost analysis using the hospital perspective. Journal of the American Geriatrics Society 1997;45(6):729-34.

\section{Cross 2010 \{published data only\}}

Cross E, Goodacre S, Team Escape Research. Patient satisfaction with chest pain unit care: findings from the Effectiveness and Safety of Chest Pain Assessment to Prevent Emergency Admissions (ESCAPE) cluster randomised trial. Emergency Medicine Journal 2010;27(10):774-8.

Cruz 2001 \{published data only\}

Cruz IG, Motilla Sanz MA, Mateos JH, Sabas AM, Sosbilla JF, Monteros BS, et al. Quality care of short stay unit relevant to critical care and emergency services. Prehospital and Disaster Medicine 2001;16(S1):S20-1.

Dallos 1981 \{published data only\} Dallos V, Mouzas GL. An evaluation of the functions of the short-stay observation ward in the accident and emergency department. British Medical Journal 1981;282(6257):37-40.

\section{Deng 2004 \{published data only\}}

Deng X, Yang DG, Wu QK, Tao YY, Nie G. A short-term clinical observation on chronic severe hepatitis B treated by Chishao Chengqi decoction. Chinese Journal of Integrated Traditional and Western Medicine on Liver Diseases 2004;14(2):67-9.

\section{Diagana 2008 \{published data only\}}

Diagana M, Salem BO, N'Diaye M, LeCornet C, Quet F, Aly AS, et al. Impact of acute unit care improving post-stroke functionality outcomes in Noukachott, Mauritania. African Journal of Neurological Sciences 2008;27:38-46.

\section{Downing 2008 \{published data only\}}

Downing H, Scott C, Clive K. Evaluation of a dedicated shortstay unit for acute medical admissions. Clinical Medicine 2008;8(1):18-20.

\section{Ekdahl 2014 \{published data only\}}

Ekdahl AW, Wirehn AB, Jaarsma T, Unosson M, Alwin J, Husberg M, et al. Caring for elderly with multimorbidity: evaluation of ambulatory geriatric unit (AGU) (the AGe-FITstudy) - a randomized controlled trial. European Geriatric Medicine 2014;5:S63-4.

\section{Ekerstad 2017 \{published data only\}}

* Ekerstad N, Karlson BW, Dahlin Ivanoff S, Landahl S, Andersson D, Heintz E, et al. Is the acute care of frail elderly patients in a comprehensive geriatric assessment unit superior to conventional acute medical care?. Clinical Interventions in Aging 2017;12:1-9.

\section{Fagerberg 2000 \{published data only\}}

Fagerberg B, Claesson L, Gosman-Hedstrom G, Blomstrand C. Effect of acute stroke unit care integrated with care continuum versus conventional treatment: a randomized 1-year study of elderly patients: the Goteborg 70+ Stroke Study. Stroke 2000;31(11):2578-84 


\section{Farkouh 1997 \{published data only\}}

Farkouh M. The effectiveness of a chest pain observation unit (CPU) in emergency triage of patients at intermediate risk for coronary events: the CHEER Trial (Chest Pain Evaluation in the ER). American Heart Journal 1997;134(1):153.

Fayas 2013 \{published data only\}

Fayas L, Polum K, Stanko H. Abstract WP252: Reduction in stroke readmission rates after implementing an emergency department-based transient ischemic attack observation unit. Stroke 2013;44(Suppl 1):AWP252.

Fung 2007 \{published data only\} Fung HT, Tsui KL, Kam CW. An overview of an emergency department short stay ward in Hong Kong. Hong Kong Journal of Emergency Medicine 2007;14(3):144-50.

Furlanetto 2014 \{published data only\} Furlanetto TW, de Souza Barcelos D, Faulhaber GAM. A short stay unit decreased mean length of stay in a hospital in south Brazil. Clinical \& Biomedical Research 2014;34(4):381-6.

\section{Gaspoz 1994 \{published data only\}}

Gaspoz JM, Lee TH, Weinstein MC, Cook EF, Goldman P, Komaroff AL, et al. Cost-effectiveness of a new short-stay unit to "rule out" acute myocardial infarction in low risk patients. Journal of the American College of Cardiology 1994;24(5):1249-59.

\section{Germain 1995 \{published data only\}}

Germain M, Knoeffel F, Wieland D, Rubenstein LZ. A geriatric assessment and intervention team for hospital inpatients awaiting transfer to a geriatric unit: a randomized trial. Aging (Milano) 1995;7(1):55-60.

\section{Ghaemmaghami 2009 \{published data only\}}

Ghaemmaghami CA, Brady WJ. Disposition from the Short Stay Unit. Short Stay Management of Chest Pain. Contemporary Cardiology. Humana Press, 2009:205-17.

\section{Goodacre 1998 \{published data only\}}

Goodacre SW. Role of the short stay observation ward in accident and emergency departments in the United Kingdom. Journal of Accident \& Emergency Medicine 1998;15(1):26-30.

\section{Goodacre 2004 \{published data only\}}

Goodacre S, Nicholl J, Dixon S, Cross E, Angelini K, Arnold K, et al. Randomised controlled trial and economic evaluation of a chest pain observation unit compared with routine care. BMJ 2004;31:254.

\section{Goodacre 2007 \{published data only\}}

Goodacre S, Cross E, Lewis C, Nicholl J, Capewell S. Effectiveness and safety of chest pain assessment to prevent emergency admissions: ESCAPE cluster randomised trial. BMJ 2007;335(7621):659-62.

\section{Harper 1988 \{published data only\}}

Harper N, McDowell DK, Turner JJ, Sharma AK. Planned shortstay admission to a geriatric unit: one aspect of respite care. Age and Ageing 1988;17(3):199-204.

\section{Harris 1991 \{published data only\}}

Harris RD, Henschke PJ, Popplewell PY, Radford AJ, Bond MJ, Turnbull RJ, et al. A randomised study of outcomes in a defined group of acutely ill elderly patients managed in a geriatric assessment unit or a general medical unit. Australian and New Zealand Journal of Medicine 1991;21(2):230-4.

Harrison 2003 \{published data only\}

Harrison J, Marshall S, Marshall P, Marshall J, Creed F. Day hospital vs. home treatment - a comparison of illness severity and costs. Social Psychiatry and Psychiatric Epidemiology 2003;38(10):541-6.

ISRCTN21800480 \{published data only\}

ISRCTN21800480. Acute medical unit comprehensive geriatric assessment intervention study. www.isrctn.com/ ISRCTN21800480 Date first received July 27th 2010. [ISRCTN21800480]

\section{Jagminas 2005 \{published data only\}}

Jagminas L, Partridge R. A comparison of emergency department versus inhospital chest pain observation units. The American Journal of Emergency Medicine 2005;23(2):111-3.

Juan 2006 \{published data only\} Juan A, Salazar A, Alvarez A, Perez J R, Garcia L, Corbella X. Effectiveness and safety of an emergency department shortstay unit as an alternative to standard inpatient hospitalisation. Emergency Medicine Journal 2006;23(11):833-7.

Juan 2010 \{published data only\} Juan PA, Ferré LC, Llopis RF, Jacob RJ, Bardés RI, Salazar SA. The short stay unit as an alternative to conventional hospitalisation in the treatment of community acquired pneumonia in the over 75 year-old population. Revista Espanola de Geriatria y Gerontologia 2010;46(4):213-6.

\section{Kam 2008 \{published data only\}}

Kam CW. Does an efficient Emergency Medicine (EM) ShortStay Unit (short-stay unit) reduce or redistribute admission. www3.ha.org.hk/haconvention/hac2008/proceedings/pdf/ Masterclass/MC1-4\%20KAM.pdf 2008. [http://www3.ha.org.hk/ haconvention/hac2008/proceedings/pdf/Masterclass/ MC1-4\%20KAM.pdf]

Kelen 2001 \{published data only\}

Kelen GD, Scheulen JJ, Hill PM. Effect of an emergency department (emergency department) managed acute care unit on emergency department overcrowding and emergency medical services diversion. Academic Emergency Medicine 2001;8(11):1095-100.

\section{Khan 1997 \{published data only\}}

Khan SA, Millington H, Miskelly FG. Benefits of an accident and emergency short stay ward in the staged hospital care of elderly patients. Journal of Accident \& Emergency medicine 1997;14(3):151-2.

\section{Koton 2005 \{published data only\}}

Koton S, Schwammenthal Y, Merzeliak O, Philips T, Tsabari R, Orion D. Management and outcome of acute stroke: differences 
between an organized short-term acute stroke unit and general medical wards. Cerebrovacular Diseases 2005;19(2):58.

\section{Llopis 2015 \{published data only\}}

Llopis RF, Ferre LC, Juan PA, Martin-Sanchez FJ, Sempere MG, Jacob RJ, et al. Spanish short-stay-units: results according to department designated to manage the unit. Emergencias 2015;27(2):109-12.

\section{Llopis 2016 \{published data only\}}

Llopis F, Ferré C, García-Lamberechts EJ, Martínez-Ortiz-deZárate M, Jacob J, González-Del-Castillo J. Are short-stay units an appropriate resource for hospitalising elderly patients with infection?. Revista de Calidad Asistencial 2016;31(6):322-8.

\section{Mahler 2013 \{published data only\}}

Mahler SA, Hiestand BC, Nwanaji-Enwerem J, Goff DC, Burke GL, Douglas $\mathrm{CL}$, et al. Reduction in observation unit length of stay with coronary computed tomography angiography depends on time of emergency department presentation. Academic Emergency Medicine 2013;20(3):231-9.

\section{Mahler 2015 \{published data only\}}

Mahler SA, Riley RF, Hiestand BC, Russell GB, Hoekstra JW, Lefebvre CW, et al. The HEART Pathway randomized trial identifying emergency department patients with acute chest pain for early discharge. Circulation: Cardiovascular Quality and Outcomes 2015;8(2):195-203.

\section{Martín-Sánchez 2014 \{published data only\}}

Martín-Sánchez FJ, Carbajosa V, Llorens P, Herrero P, Jacob J, Pérez-Dura MJ, et al. Prolonged hospitalization in patients admitted for acute heart failure in the short stay unit (EPICAUCE study): study of associated factors. Medicina Clinica 2014;143(6):245-51.

\section{Michael 2014 \{published data only\}}

Michael AB, Ijaola F. P406: Frail elderly short stay unit; a model of emergency care for older people. European Geriatric Medicine 2014;5:S209.

\section{Miller 2011 \{published data only\}}

Miller CD, Hwang W, Case D, Hoekstra JW, Lefebvre C, Blumstein $\mathrm{H}$, et al. Stress CMR imaging observation unit in the emergency department reduces 1-year medical care costs in patients with acute chest pain: a randomized study for comparison with inpatient care. Journal of the American College of Cardiology Cardiovascular Imaging 2011;4(8):862-70.

Miller 2012 \{published data only\}

Miller CD, Hoekstra JW, Lefebvre C, Blumstein H, Hamilton CA, Harper EN, et al. Provider-directed imaging stress testing reduces health care expenditures in lower-risk chest pain patients presenting to the emergency department. Circulation: Cardiovascular Imaging 2012;5(1):111-8.

\section{Mitchell 2009 \{published data only\}}

Mitchell MD, Williams K. Observation units (structured abstract). Health Technology Assessment Database 2009, issue 3.
Muñoz 2006 \{published data only\}

Muñoz RN, Méndez BM, Conthe GP, Audibert ML. Management of heart failure in short stay medical unit: mean stay, frequency and predictors of readmission. Medicina Clinica 2006;127(13):516-7.

\section{Navarrete 2016 \{published data only\}}

Navarrete G. Reducing delirium length and hospital stay in hospitalized elders: a randomized, controlled trial. Journal of the American Geriatrics Society 2016;64:S34.

\section{NCT02421133 \{published data only\}}

NCT02421133. Impact of a transitional care program on 30-day hospital readmissions for elderly patients discharged from a short stay geriatric ward. Clinicaltrials.gov Date first received April 20th 2015. [NCT02421133]

\section{Ross 2004 \{published data only\}}

Ross MA, Bradley D, Dresselhous A. The role of an emergency department observation unit In a clinical pathway for atrial fibrillation. Critical Pathways in Cardiology 2004;3(1):8-12.

Rubenstein 1984 \{published data only\}

Rubenstein LZ, Josephson KR, Wieland GD, English PA, Sayre JA, Kane RL. Effectiveness of a geriatric evaluation unit. A randomized clinical trial. New England Journal of Medicine 1984;311(26):1664-70.

\section{Rubenstein 1988 \{published data only\}}

Rubenstein LZ, Wieland GD, Josephson KR, Rosbrook B, Sayre J, Kane RL. Improved survival for frail elderly inpatients on a geriatric evaluation unit (GEU): who benefits?. Journal of Clinical Epidemiology 1988;41(5):441-9.

Salazar 2006 \{published data only\}

Salazar A, Juan A, Ballbe R, Corbella X. Short-stay unit: an effective alternative to in-hospital admission for acute COPD exacerbation. American Journal of Emergency Medicine 2006;25(4):486-7.

\section{References to ongoing studies}

NCT03302910 \{unpublished data only\}

NCT03302910. Short stay unit vs hospitalization in acute heart failure (SSU-AHF). clinicaltrials.gov/ct2/show/NCT03302910 Date first received October 5th 2017. [NCT03302910]

\section{Additional references \\ ACEP 2008}

American College of Emergency Physicians. ACEP Task Force report on boarding. Emergency department crowding: high-impact solutions (2008). www.acep.org/workarea/ DownloadAsset.aspx?id=50026 (accessed 15 March 2016).

\section{Baker 2004}

Baker GR, Norton PG, Flintoft V, Blais R, Brown A, Cox J, et al. The Canadian Adverse Events Study: the incidence of adverse events among hospital patients in Canada. Canadian Medical Association Journal 2004;170:1678-86. 


\section{Brennan 1991}

Brennan TA, Leape LL, Laird NM, Hebert L, Localio AR, Lawthers AG, et al. Incidence of adverse events and negligence in hospitalized patients. Results of the Harvard Medical Practice Study I. New England Journal of Medicine 1991;324:370-6.

\section{CDC 2013}

Centers for Disease Control and Prevention/National Center for Health Statistics. Death and Mortality. NCHS FastStats. www.cdc.gov/nchs/fastats/deaths.htm (2013) (accessed 20 December 2015)

\section{Christensen 2009}

Christensen KL, Holman RC, Steiner CA, Sejvar JJ, Stoll BJ, Schonberger LB. Infectious disease hospitalizations in the United States. Clinical Infectious Diseases 2009;49:1025-35.

\section{Classen 1997}

Classen DC, Pestotnik SL, Evans RS, Lloyd JF, Burke JP. Adverse drug events in hospitalized patients. Excess length of stay, extra costs, and attributable mortality. Journal of the American Medical Association 1997;277:301-6.

\section{Covidence 2015 [Computer program]}

Veritas Health Innovation. Covidence. Melbourne, Australia: Veritas Health Innovation, Assessed December 1st 2016.

\section{Cowling 2014}

Cowling TE, Soljak NA, Bell D, Majeed A. Emergency hospital admissions via accident and emergency departments in England: time trend, conceptual framework and policy implications. Journal of the Royal Society of Medicine 2014;107:432-8

\section{Cullen 2011}

Cullen MW, Reeder GS, Farkouh ME, Kopecky SL, Smars PA, Behrenbeck TR, et al. Outcomes in patients with chest pain evaluated in a chest pain unit: the chest pain evaluation in the emergency room study cohort. American Heart Journal 2011;161(5):871-7.

\section{Daly 2003}

Daly S, Campbell DA, Cameron PA. Short-stay units and observation medicine: a systematic review. Medical Journal of Australia 2003;178:559-63.

\section{Damiani 2011}

Damiani G, Pinnarelli L, Sommella L, Vena V, Magrini P, Ricciardi W. The Short Stay Unit as a new option for hospitals: a review of the scientific literature. International Medical Journal of Experimental and Clinical Research 2011;17:SR15-9.

\section{Dang-Tan 2015}

Dang-Tan T, Ismaila A, Zhang S, Zarotsky V, Bernauer M. Clinical, humanistic, and economic burden of chronic obstructive pulmonary disease (COPD) in Canada: a systematic review. BMC Research Notes 2015;8:464.

\section{Deeks 2011}

Deeks JJ, Higgins JPT, Altman DG (editors). Chapter 9: Analysing data and undertaking meta-analyses. In: Higgins JPT, Green
S (editors). Cochrane Handbook for Systematic Reviews of Interventions Version 5.1.0 (updated March 2011). The Cochrane Collaboration, 2011. Available from handbook.cochrane.org.

\section{DerSimonian 1986}

DerSimonian R, Laird N. Meta-analysis in clinical trials. Controlled Clinical Trials 1986;7:177-88.

\section{EFIM 2007}

European Federation of Internal Medicine. What is internal medicine?. www.efim.org/about/what-internal-medicine (2007) (accessed 26 November 2015).

\section{Endnote X7 [Computer program]}

Clarivate Analytics. Endnote X7. Clarivate Analytics, 2015.

\section{EPOC 2013a}

Effective Practice, Organisation of Care (EPOC). What outcomes should be reported in Cochrane Effective Practice and Organisation of Care (EPOC) reviews?. Oslo: Norwegian Knowledge Centre for the Health Services; 2013. Available at: epoc.cochrane.org/epoc-specific-resources-review-authors.

\section{EPOC 2013b}

Effective Practice, Organisation of Care (EPOC). Analysis in EPOC reviews. Oslo: Norwegian Knowledge Centre for the Health Services; 2013. Available at: epoc.cochrane.org/epoc-specificresources-review-authors.

\section{EPOC 2013C}

Effective Practice, Organisation of Care (EPOC). What studies should be included in the Characteristics of excluded studies table?. EPOC resources for review authors. Oslo: Norwegian Knowledge Centre for the Health Services; 2013. Available at: epoc.cochrane.org/epoc-specific-resources-reviewauthors.

\section{EPOC 2013d}

Effective Practice, Organisation of Care (EPOC). EPOC worksheets for preparing a Summary of Findings (SoF) table using GRADE. EPOC resources for review authors. Oslo: Norwegian Knowledge Centre for the Health Services; 2013. Available at: epoc.cochrane.org/epoc-specific-resourcesreview-authors.

\section{EPOC 2013e}

Effective Practice, Organisation of Care (EPOC). What are explanatory factors and why should they be included in protocols?. EPOC resources for review authors. Oslo: Norwegian Knowledge Centre for the Health Services; 2013. Available at: epoc.cochrane.org/epoc-specific-resources-review-authors.

\section{EPOC 2013f}

Effective Practice, Organisation of Care (EPOC). Results should not be reported as statistically significant or statistically nonsignificant. EPOC resources for review authors. Oslo: Norwegian Knowledge Centre for the Health Services 2013. Available at: epoc.cochrane.org/epoc-specific-resources-review-authors.

\section{EPOC 2015a}

Effective Practice, Organisation of Care (EPOC). Suggested risk of bias criteria for EPOC reviews. EPOC resources for review 
authors. Oslo: Norwegian Knowledge Centre for the Health Services; 2015. Available at: epoc.cochrane.org/epoc-specificresources-review-authors.

\section{Feng 2012}

Feng Z, Wright B, Mor V. Sharp rise in Medicare enrollees being held in hospitals for observation raises concerns about causes and consequences. Health Affairs 2012;31:1251-9.

\section{Galipeau 2015}

Galipeau J, Pussegoda K, Stevens A, Brehaut JC, Curran J, Forster AJ, et al. Effectiveness and safety of short-stay units in the emergency department: a systematic review. Academic Emergency Medicine 2015;22:893-907.

\section{Gaspoz 1991}

Gaspoz JM, Lee TH, Cook EF, Weisberg MC, Goldman L. Outcome of patients who were admitted to a new short-stay unit to "ruleout" myocardial infarction. American Journal of Cardiology 1991;68:145-9.

\section{Gerteis 2014}

Gerteis J, Izrael D, Deitz D, LeRoy L, Ricciardi R, Miller T, Basu J (AHRQ). Multiple chronic conditions chartbook. www.ahrq.gov/ sites/default/files/wysiwyg/professionals/prevention-chroniccare/decision/mcc/mccchartbook.pdf (2014) (accessed 18 December 2015).

\section{GRADEpro GDT 2015 [Computer program]}

McMaster University (developed by Evidence Prime. GRADEpro GDT. Hamilton (ON): McMaster University (developed by Evidence Prime, 2015

\section{Guyatt 2008}

Guyatt GH, Oxman AD, Vist G, Kunz R, Falck-Ytter Y, AlonsoCoello $P$, et al. GRADE Working Group. Rating quality of evidence and strength of recommendations GRADE: an emerging consensus on rating quality of evidence and strength of recommendations. BMJ 2008;336:924-6.

\section{Higgins 2003}

Higgins JPT, Thompson SG, Deeks JJ, Altman DG. Measuring inconsistency in meta-analyses. BMJ 2003;327:557-60.

\section{Higgins 2011a}

Higgins JPT, Green S (editors). Cochrane Handbook for Systematic Reviews of Interventions Version 5.1.0 (updated March 2011). The Cochrane Collaboration, 2011. Available from www.handbook.cochrane.org.

\section{Higgins 2011b}

Higgins JPT, Altman DG, Sterne JAC (editors). Chapter 8: Assessing risk of bias in included studies. In: Higgins JPT, Green S (editors). Cochrane Handbook for Systematic Reviews of Interventions Version 5.1.0 (updated March 2011). The Cochrane Collaboration, 2011. Available from www.handbook.cochrane.org.

\section{Higgins 2011c}

Higgins JPT, Deeks JJ, Altman DG (editors). Chapter 16: Special topics in statistics. In: Higgins JPT, Green S (editors), Cochrane
Handbook for Systematic Reviews of Interventions Version 5.1.0 (updated March 2011). The Cochrane Collaboration, 2011. Available from handbook.cochrane.org.

\section{Higgins 2011d}

Higgins JPT, Deeks JJ (editors). Chapter 7: Selecting studies and collecting data. In: Higgins JPT, Green S (editors), Cochrane Handbook for Systematic Reviews of Interventions Version 5.1.0 (updated March 2011). The Cochrane Collaboration, 2011. Available from handbook.cochrane.org.

\section{ICH-GCP 1997}

International Conference on Harmonisation. Good clinical practice: guidelines. www.ich.org/products/guidelines/efficacy/ article/efficacy-guidelines.html (1997) (accessed 16 November 2015).

\section{Inouye 1990}

Inouye SK, Van Dyck CH, Alessi CA, Balkin S, Siegal AP, Horwitz RI. Clarifying confusion: the confusion assessment method. A new method for detection of delirium. Annals of Internal Medicine 1990;113:941-8.

\section{Jakobsen 2014}

Jakobsen JC, Wetterslev J, Winkel P, Lange T, Gluud C. Thresholds for statistical and clinical significance in systematic reviews with meta-analytic methods. BMC Medical Research Methodology 2014;14:120.

\section{Jibrin 2008}

Jibrin I, Hamirani YS, Mitikiri N, Ozdegirmenci H, Wentz C, Bahr RD. Maryland's first inpatient chest pain short stay unit as an alternative to emergency room-based observation unit. Critical Pathways in Cardiology 2008;7:35-42.

\section{Kenny 2015}

Kenny RA, Brignole M, Dan GA, Deharo JC, Van Dijk JG, Doherty $C$, et al. Syncope Unit: rationale and requirement - the European Heart Rhythm Association position statement endorsed by the Heart Rhythm Society. Europace 2015;17:1325-40.

\section{Laskin 1972}

Laskin DM. The short-stay surgical facility: something oldsomething new. Journal of Oral Surgery 1972;30:394.

\section{Lawton 1969}

Lawton MP, Brody EM. Assessment of older people: selfmaintaining and instrumental activities of daily living. Gerontologist 1969;9:179-86.

\section{Liberati 2009}

Liberati A, Altman DG, Tetzlaff J, Mulrow C, Gotzsche PC, loannidis JP, et al. The PRISMA statement for reporting systematic reviews and meta-analyses of studies that evaluate health care interventions: explanation and elaboration. PLOS Medicine 2009;6:e1000100.

\section{Lowthian 2011}

Lowthian JA, Curtis AJ, Cameron PA, Stoelwinder JU, Cooke MW, McNeil JJ. Systematic review of trends in emergency 
department attendances: an Australian perspective. Emergency Medicine Journal 2011;28:373-7.

\section{Mantel 1959}

Mantel N, Haenszel W. Statistical aspects of the analysis of data from retrospective studies of disease. Journal of the National Cancer Institute 1959;22:719-48.

\section{Marco 2010}

Marco J, Barba R, Plaza S, Losa JE, Canora J, Zapatero A. Analysis of the mortality of patients admitted to internal medicine wards over the weekend. American Journal of Medical Quality 2010;25(4):312-8.

\section{McGowan 2016}

McGowan J, Sampson M, Salzwedel DM, Cogo E, Foerster V, Lefebvre C. PRESS Peer Review of Electronic Search Strategies: 2015 guideline statement. Journal of Clinical Epidemiology 2016;75:40-6. [DOI: https://doi.org/10.1016/ j.jclinepi.2016.01.021]

\section{Mosely 2013}

Moseley MG, Hawley MP, Caterino J. Emergency department observation unit and the older patient. Clinics in Geriatric Medicine 2013;29(1):1-26.

\section{Pfeiffer 1978}

Pfeiffer E. Multidimensional functional assessment: the OARS methodology: a manual / Duke University, Center for the Study of Aging and Human Development. 2nd Edition. Duke University, Center for the Study of Aging and Human Development, 1978. [Libraries Australia ID 1994215]

\section{Pfuntner 2013}

Pfuntner A, Wier LM, Stocks C, AHRQ. Most frequent conditions in U.S. hospitals, 2010. www.hcup-us.ahrq.gov/reports/ statbriefs/sb148.pdf (2013) (accessed 15 March 2016).

\section{Reid 2016}

Reid L, Dinesen LC, Jones MC, Morrison ZJ, Weir CH, Lone NI. The effectiveness and variation of acute medical units: a systematic review. International Journal for Quality in Health Care 2016;28(4):433-46.

\section{RevMan 2014 [Computer program]}

Nordic Cochrane Centre, The Cochrane Collaboration. Review Manager 5 (RevMan 5). Version 5.3. Copenhagen: Nordic Cochrane Centre, The Cochrane Collaboration, 2014.

\section{Ross 2013}

Ross MA, Hockenberry JM, Mutter R, Barrett M, Wheatley M, Pitts SR. Protocol-driven emergency department observation units offer savings, shorter stays, and reduced admissions. Health Affairs 2013;32:2419-56.

\section{Russell 2014}

Russell PT, Hakendorf P, Thompson CH. A general medical shortstay unit is not more efficient than a traditional model of care. Medical Journal of Australia 2014;200:482-4.

\section{Rydman 1998}

Rydman RJ, Isola ML, Roberts RR, Zalenski RJ, McDermott MF, Murphy DG, et al. Emergency department observation unit versus hospital inpatient care for a chronic asthmatic population: a randomized trial of health status outcome and cost. Medical Care 1998;36(4):599-609.

\section{Rydman 1999}

Rydman RJ, Roberts RR, Albrect GL, Zalenski RJ, McDermott MF. Patient satisfaction with an emergency department asthma observation unit. Academic Emergency Medicine 1999;6(3):178-83.

\section{Salazar 2007}

Salazar A, Juan A, Ballbe R, Corbella X. Emergency short-stay unit as an effective alternative to in-hospital admission for acute chronic obstructive pulmonary disease exacerbation. American Journal of Emergency Medicine 2007;25:486-7.

\section{Savović 2012}

Savović J, Jones HE, Altman DG, Harris RJ, Jüni P, Pildal J, et al. Influence of reported study design characteristics on intervention effect estimates from randomized, controlled trials. Annals of Internal Medicine 2012;18(6):429-38.

\section{Schünemann 2011}

Schünemann HJ, Oxman AD, Higgins JPT, Vist GE, Glasziou P, Guyatt GH. Chapter 11: Presenting results and 'Summary of findings' tables. In: Higgins JPT, Green S (editors), Cochrane Handbook for Systematic Reviews of Interventions Version 5.1.0 (updated March 2011). The Cochrane Collaboration, 2011. Available from handbook.cochrane.org.

\section{Scott 2009}

Scott I, Vaughan L, Bell D. Effectiveness of acute medical units in hospitals: a systematic review. International Journal for Quality in Health Care 2009;21:397-407.

\section{Spanjersberg 2011}

Spanjersberg W, Reurings J, Keus F, Van Laarhoven C. Fast track surgery versus conventional recovery strategies for colorectal surgery. Cochrane Database of Systematic Reviews 2011, Issue 2. [DOI: 10.1002/14651858.CD007635.pub2]

\section{Sprivulis 2006}

Sprivulis P, Da Silva J, Jacobs I, Frazer A, Jelinek G. The association between hospital overcrowding and mortality among patients admitted via Western Australian emergency departments. Medical Journal Australia 2006;184:208-12.

\section{Sterne 2011a}

Sterne JAC, Egger M, Moher D (editors). Chapter 10: Addressing reporting biases. In: Higgins JPT, Green S (editors). Cochrane Handbook for Systematic Reviews of Intervention. Version 5.1.0 (updated March 2011). The Cochrane Collaboration, 2011. Available from handbook.cochrane.org.

\section{Sterne 2011b}

Sterne JA, Sutton AJ, Ioannidis JP, Terrin N, Jones DR, Lau J, et al. Recommendations for examining and interpreting funnel 
plot asymmetry in meta-analyses of randomised controlled trials. BMJ 2011;343:d4002.

\section{Strøm 2017b}

Strøm C, Mollerup TK, Kromberg LS, Rasmussen LS, Schmidt TA. Hospitalisation in an emergency department short-stay unit compared to an internal medicine department is associated with fewer complications in older patients - an observational study. Scandinavian Journal of Trauma Resuscitation and Emergency Medicine 2017;25(1):80.

\section{Thomas 2000}

Thomas EJ, Studdert DM, Burstin HR, Orav E, Zeena T, Williams EJ, et al. Incidence and types of adverse events and negligent care in Utah and Colorado. Medical Care 2000;38:261-71.

\section{UN 2013}

United Nations. World Population Ageing. www.un.org/en/ development/desa/population/publications/pdf/ageing/ WorldPopulationAgeing2013.pdf (accessed 15 March 2016).

\section{Vries 2008}

Vries EN, Ramratten MA, Smorenburg SM, Gouma DJ, Boermeester MA. The incidence and nature of in-hospital adverse events: a systematic review. Quality \& Safety in Health Care 208;17:216-23.

\section{Ware 1992}

Ware JE, Sherbourne CD. The MOS 36-item short-form health survey (SF-36). I. Conceptual framework and item selection. Medical Care 1992;30:473-83.

\section{CHARACTERISTICS OF STUDIES}

Characteristics of included studies [ordered by study ID]

\section{WHO 2011}

World Health Organization (WHO). What is the impact of noncommunicable diseases on National Health Expenditures: a synthesis of available data. www.who.int/healthsystems/ NCDdiscussionpaper3.pdf (2011) (accessed 15 March 2016).

\section{Wind 2006}

Wind J, Polle SW, Fung KJ, Dejong C, von Meyenfeldt MF, Ubbink DT, et al. Systematic review of enhanced recovery programmes in colonic surgery. British Journal of Surgery 2006;93:800-9.

\section{Wolff 2002}

Wolff JL, Starfield B, Anderson G. Prevalence, expenditures, and complications of multiple chronic conditions in the elderly. Archives of Internal Medicine 2002;162:2269-76.

\section{References to other published versions of this review Strøm 2016}

Strøm C, Fabritius M, Rasmussen LS, Schmidt TA, Jakobsen JC. Hospitalisation in short-stay units for internal medicine diseases and conditions. Cochrane Database of Systematic Reviews 2016, Issue 9. [DOI: 10.1002/14651858.CD012370]

* Indicates the major publication for the study

\section{Chivite 2008}

Methods Parallel randomised trial

Aim of trial: to compare SSU hospitalisation with hospitalisation in an internal medicine service in older participants with decompensated heart failure

Setting: single-centre trial at an urban, tertiary, public university hospital, Barcelona, Spain, November 2001-March 2004

Number of nurses/bed: $4 / 24$ beds in intervention group vs $6 / 48$ beds in usual-care group

Number of physicians/bed: $1 / 24$ beds in intervention group vs 5 staff physicians +5 residents/unclear number of participants in usual-care group

Type of SSU: multipurpose unit

Inclusion criteria: age > 65 years, acute decompensated heart failure, clinical stability, moderate comorbidity, moderate disability

Exclusion criteria: secondary heart failure diagnosis (defined to be ACSs, severe valve disease, pericardial disease, or isolated cor pulmonale), estimated survival $<6$ months, severe cognitive impairment, severe functional impairment, unstable clinical condition after initial ED management (defined to be hypotension, tachycardia, electrolyte imbalances, acute kidney failure, need for vasoactive drugs)

Sample size was not calculated, consecutive participants were included until the end of the funding 
Chivite 2008 (Continued)

Numbers recruited: 139; SSU: 70; internal medicine services: 69

Participants analysed: various numbers of participants lost to follow-up depending on outcome and time of assessment

Mean age: 77 (SD 6.3) in intervention group vs 78.9 (SD 6.6) in usual-care group

Male: 35 (54\%) in intervention group vs 35 (51\%) in usual-care group

NYHA: 2.6 (SD 0.6) in intervention group vs 2.5 (SD 0.5) in usual-care group

CCI: 2.5 (SD 1.3) in intervention group vs 2.2 (SD 1.2) in usual-care group

\begin{tabular}{|c|c|}
\hline Interventions & $\begin{array}{l}\text { Intervention: treatment in SSU, no standardised treatment protocol, but aimed at early removal of iv } \\
\text { lines and early discharge planning, urinary catheters and bed rest was discouraged } \\
\text { Usual care: hospitalisation in internal medicine services, no further description available }\end{array}$ \\
\hline \multirow[t]{3}{*}{ Outcomes } & $\begin{array}{l}\text { Outcome hierarchy was not available in the methods section, but trial authors stated that they investi- } \\
\text { gated } 3 \text { primary outcomes: }\end{array}$ \\
\hline & $\begin{array}{l}\text { Primary outcomes: 1-year heart failure-related hospital readmission, 1-year heart failure-related ED re- } \\
\text { visit, and 1-year all-cause mortality. }\end{array}$ \\
\hline & $\begin{array}{l}\text { Secondary outcomes: index admission length of stay in hospital, QoL (change in Minnesota Living With } \\
\text { Heart Failure Quality of Life Scale and European Heart Failure Self-Care Behaviour Scale), functional } \\
\text { status (Barthel Index and OARS-IADL), exercise capacity (NYHA, } 6 \text { minutes walking test), and total hos- } \\
\text { pital costs }\end{array}$ \\
\hline \multirow[t]{5}{*}{ Notes } & $\begin{array}{l}\text { Defined time limit of maximum stay in the SSU: } 5 \text { days, but transfers were not compulsory after dead- } \\
\text { line }\end{array}$ \\
\hline & Follow-up: 1 year \\
\hline & Trial registration: no registration \\
\hline & $\begin{array}{l}\text { Study was only available in abstract format, details on trials including bias assessment were obtained } \\
\text { after contact with trial authors. }\end{array}$ \\
\hline & Received additional information from trial authors by 6 March 2017 \\
\hline
\end{tabular}

Risk of bias

\begin{tabular}{|c|c|c|}
\hline Bias & Authors' judgement & Support for judgement \\
\hline $\begin{array}{l}\text { Random sequence genera- } \\
\text { tion (selection bias) }\end{array}$ & Low risk & $\begin{array}{l}\text { Computerised random number generation (information following contact with } \\
\text { the trial authors) }\end{array}$ \\
\hline $\begin{array}{l}\text { Allocation concealment } \\
\text { (selection bias) }\end{array}$ & Low risk & $\begin{array}{l}\text { Opaque sealed envelopes (information following contact with the trial au- } \\
\text { thors) }\end{array}$ \\
\hline $\begin{array}{l}\text { Baseline outcome mea- } \\
\text { surement }\end{array}$ & Unclear risk & $\begin{array}{l}\text { No baseline measure of outcomes QoL and activities of daily living obtained } \\
\text { from trial authors }\end{array}$ \\
\hline Baseline characteristics & Low risk & No differences between groups (detailed information from the trial authors) \\
\hline $\begin{array}{l}\text { Blinding of outcome as- } \\
\text { sessment (detection bias) } \\
\text { All outcomes }\end{array}$ & High risk & No blinding (information following contact with the trial authors) \\
\hline $\begin{array}{l}\text { Incomplete outcome data } \\
\text { (attrition bias) }\end{array}$ & High risk & $\begin{array}{l}\text { Sample size was not calculated, consecutive participants were included until } \\
\text { the end of the funding. Various numbers of participants lost to follow-up de- }\end{array}$ \\
\hline
\end{tabular}


Chivite 2008 (Continued)

All outcomes pending on outcome. $<15 \%$ of included participants were lost to follow-up for mortality and hospital readmission, but $>15 \%$ of included participants were lost to follow-up for quality-of-life measurements. Unclear how many participants were analysed for costs. No participants were lost to follow-up for length of stay in hospital (information following contact with trial authors).

\begin{tabular}{lll}
\hline Contamination & Unclear risk & Unclear if there was risk of contamination \\
\hline $\begin{array}{l}\text { Selective reporting (re- } \\
\text { porting bias) }\end{array}$ & High risk & $\begin{array}{l}\text { The trial was not registered a in a trial register, trial was conducted before } \\
2005(2001-2004) \text {. No protocol was available. All outcomes noted in abstract } \\
\text { were reported in detail after contact with trial authors. }\end{array}$ \\
\hline Other bias & High risk & $\begin{array}{l}\text { Sample size was not calculated, consecutive participants were included until } \\
\text { the end of the funding. Dr. Salazar had written more than } 3 \text { papers on SSUs, } \\
\text { academic bias may be present. The trial was solely reported in an abstract, not } \\
\text { published. }\end{array}$ \\
\hline
\end{tabular}

\begin{tabular}{|c|c|c|}
\hline $\begin{array}{l}\text { Overall risk of bias } \\
\text { All outcomes }\end{array}$ & High risk & $\begin{array}{l}\text { High risk of bias and/or unclear risk of bias in one or more domains: blinding of } \\
\text { outcome assessment, incomplete outcome data, or selective reporting }\end{array}$ \\
\hline
\end{tabular}

\section{Decker 2008}

Parallel randomised trial
Aim of trial: to compare outcomes in care of participants with acute onset AF randomised to observa-
tion unit with electrical cardioversion or routine inpatient admission
Setting: single-centre trial conducted in an urban, tertiary, private non-profit hospital, Rochester, Min-
nesota, USA, September 1999-December 2002
Number of nurses/bed: unclear
Number of physicians/bed: unclear
Type of SSU: multipurpose unit

Participants
Inclusion criteria: adult participants $>18$ years presenting to ED with $\mathrm{AF}$ of $<48 \mathrm{~h}$ duration, without
haemodynamic instability, or other conditions requiring hospitalisation
Exclusion criteria: $\mathrm{AF}$ of $>48 \mathrm{~h}$ duration, uncertain duration of symptoms, haemodynamic instability
(systolic $\mathrm{BP}<90 \mathrm{mmHg}$, diastolic $\mathrm{BP}<50 \mathrm{mmHg}$, pulse rate of $\geq 130 \mathrm{bpm}$ after attempts to rate con-
trol, known intracardiac thrombus, class IV congestive heart failure, ejection fraction $<30 \%$, chest pain
consistent with class IV angina, acute MI within 4 weeks before $\mathrm{AF}$ onset, stroke or transient neurolog-
ic ischaemic attack in the past 3 months, previous unsuccessful electrical cardioversion of AF or active
medical problems other than AF such as unstable angina, pneumonia, transient neurologic ischaemic
attacks, and strokes requiring inpatient evaluation, patients residing outside of Olmsted County or its
surrounding 9 counties

Numbers recruited: 153,75 to the intervention group vs 78 to the usual-care group

Participants analysed for the primary outcome: 153

Mean age: 58 (SD 18) in intervention group vs 59 (SD 16) in usual-care group

Male: 40 (53\%) in intervention group vs 54 (69\%) in usual-care group 
Decker 2008 (Continued)

diac monitoring for $6 \mathrm{~h}$. Those still in AF were sedated and electrically cardioverted and observed for a further period of $2 \mathrm{~h}$. Those in sinus rhythm after the 2-h observation period were discharged home, with cardiology follow-up arranged within 3 days. Those remaining in AF after unsuccessful attempts to electrically cardiovert were admitted to the hospital cardiology service

Usual care: routine hospital care with an ECG and routine laboratory investigations in the ED, administration of an iv calcium channel blocker or a blocker for rate control, initiation of heparin infusion, and admission to a monitored bed on the cardiology service

Intervention in details: 8-h ED observation unit protocol included recording of an ECG, chest radiograph, and routine laboratory investigations, including electrolyte levels, CBC, and glucose level. This was followed by pharmacologic pulse rate control using a calcium channel blocker or alpha-blocker. Rate control was defined as a ventricular response $<100 \mathrm{bpm}$ at rest. All participants received continuous cardiac monitoring and were reassessed after $6 \mathrm{~h}$. Those still in AF were sedated and electrically cardioverted with the Physio Lifepak 6 (Medtronic Inc., Minneapolis, MN; before 2001) or the Zoll M Series Biphasic Manual device (Zoll Medical Corporation, Burlington, MA; after 2001) for correction of $\mathrm{AF}$ and observed for a further period of $2 \mathrm{~h}$. Those in sinus rhythm after the 2-h observation period were discharged home, with cardiology follow-up arranged within 3 days. Participants who were enrolled in the trial in the evening were observed overnight and cardioverted between 7 and 9 am Study participants treated in the ED observation unit were not given any antiarrhythmic on discharge and were not anticoagulated. Those remaining in AF after unsuccessful attempts to electrically cardiovert were admitted to the hospital cardiology service. All care, including initial evaluation, ED observation unit care, procedural sedation, and cardioversion, was overseen by the emergency medicine attending physician on duty

Outcomes Primary outcome: conversion to sinus rhythm or rate control at the completion of initial ED observation unit or hospital stay

Secondary outcomes: recurrence of AF and adverse events (subsequent MI, congestive heart failure, stroke, or death). Further utilisation of healthcare resources was measured by further recurrent visits to the hospital

Notes Defined time limit of maximum stay in the SSU: $8 \mathrm{~h}$

Follow-up: 6 months

Trial registration: none

Received additional information from trial authors by 27 February 2017

\section{Risk of bias}

\begin{tabular}{|c|c|c|}
\hline Bias & Authors' judgement & Support for judgement \\
\hline $\begin{array}{l}\text { Random sequence genera- } \\
\text { tion (selection bias) }\end{array}$ & Low risk & $\begin{array}{l}\text { Random sequence generation provided by a remote, designated randomisa- } \\
\text { tion centre not involved in participant care }\end{array}$ \\
\hline $\begin{array}{l}\text { Allocation concealment } \\
\text { (selection bias) }\end{array}$ & Low risk & $\begin{array}{l}\text { Allocation concealment provided by a remote, designated randomisation cen- } \\
\text { tre not involved in participant care }\end{array}$ \\
\hline $\begin{array}{l}\text { Baseline outcome mea- } \\
\text { surement }\end{array}$ & Low risk & Baseline outcome measurement not relevant \\
\hline Baseline characteristics & Unclear risk & $\begin{array}{l}\text { Higher proportion of female participants in intervention group ( } 47 \% \text { vs } 31 \%) \text {, } \\
\text { may or may not have affected outcomes }\end{array}$ \\
\hline $\begin{array}{l}\text { Blinding of outcome as- } \\
\text { sessment (detection bias) } \\
\text { All outcomes }\end{array}$ & Unclear risk & $\begin{array}{l}\text { Assessment method was by medical record review, and if necessary telephone } \\
\text { calls. Trial authors informed that outcome assessors were blinded for treat- } \\
\text { ment allocation, but it seems unlikely that the medical record review would } \\
\text { not reveal the unit of allocation }\end{array}$ \\
\hline
\end{tabular}


Decker 2008 (Continued)

Incomplete outcome data Low risk Clearly described that no participants were lost to follow-up, and no with(attrition bias) drawals

All outcomes

\begin{tabular}{lll}
\hline Contamination & High risk & Participants in intervention group were transferred to inpatient care if needed \\
\hline $\begin{array}{l}\text { Selective reporting (re- } \\
\text { porting bias) }\end{array}$ & High risk & $\begin{array}{l}\text { No trial registration. No protocol was described. Trial authors described all } \\
\text { outcomes in outcome section as defined in the methods section }\end{array}$ \\
\hline Other bias & Low risk & $\begin{array}{l}\text { The trial appeared to be free of other components that could put it at risk of } \\
\text { bias }\end{array}$ \\
\hline $\begin{array}{l}\text { Overall risk of bias } \\
\text { All outcomes }\end{array}$ & High risk & $\begin{array}{l}\text { High risk of bias and/or unclear risk of bias in one or more domains: blinding of } \\
\text { outcome assessment, incomplete outcome data, or selective reporting }\end{array}$ \\
\hline
\end{tabular}

Farkouh 1998

Parallel randomised trial
Aim of trial: to compare the safety, efficacy, and use of resources of a CPU with those of routine hospi-
tal admission for participants with unstable angina who were at intermediate risk for cardiovascular
events in the long term
Setting: single-centre trial at an urban, tertiary, public hospital, Rochester, Minnesota, USA, November
1995-March 1997
Number of nurses/bed: unclear
Number of physicians/bed: unclear
Type of SSU: specialised unit

Participants

People with chest pain that met the criteria for unstable angina and intermediate risk for cardiovascular events.

Inclusion criteria: age $\geq 18$ years, unstable angina, and intermediate risk for cardiovascular events (defined by presence of angina at rest for at least 20 min or unresponsive to nitroglycerin, dynamic ECG Twave inversions with angina, nocturnal angina, or new onset of Canadian Cardiovascular Society classification III or IV angina 2 weeks before presentation)

Exclusion criteria: participants were excluded if they had ST-segment elevation on the ECG, an obvious noncardiac cause of the chest pain, unstable angina associated with a low or high risk, or a coexisting condition requiring hospitalisation.

Numbers recruited: 424,212 to the intervention group vs 212 to the usual-care group

Participants analysed for the primary outcome:

For the long-term follow-up, 407 participants were analysed (trial authors excluded 17 participants due to lack of consent to obtain surveillance data, 8 in the intervention group, and 9 in the usual-care group)

Mean age: 57.7 (SD 1) in intervention group vs 59.2 (SD 1) in usual-care group

Male: $56.1 \%$ in intervention group vs $55.7 \%$ in usual-care group

Interventions Intervention: the CPU consisted of 4 beds in a separate area of the ED. It was equipped with event monitors and continuous ST-segment monitoring and was staffed by a full-time nurse. Care in the CPU was standardised due to a pre-defined protocol (scheduled measurement of CK-MB levels, observation for 
Farkouh 1998 (Continued)

minimum 6 h, 325 mg of aspirin, cardiac function study (treadmill testing or nuclear stress studies, follow-up appointment at $72 \mathrm{~h}$ after discharge by staff-cardiologist))

Usual care: non-standardised treatment in a monitored bed under the care of the cardiology service. This service consisted of a team of internal medicine residents or cardiology fellows under the supervision of a staff cardiologist.

Intervention detail: the total level of creatine kinase MB isoenzyme (CK-MB) was measured at the time points 0,2 , and $4 \mathrm{~h}$ after randomisation. If the CK-MB level was elevated at any time, participants were admitted to the cardiology service or to the coronary care unit with a presumptive diagnosis of MI. Participants were also admitted from the CPU to the hospital if they had symptoms of recurrent chest pain consistent with recurrent unstable angina or important ventricular dysrhythmia or had another medical condition warranting admission. All the participants randomly assigned to the CPU were observed for a minimum of $6 \mathrm{~h}$, and all received $325 \mathrm{mg}$ of aspirin. The decision whether to administer iv heparin was made by the ED physician according to clinical criteria. For all participants who 'passed' (completed) the observation period in the CPU, a cardiac-function study was performed at the end of the observation period. A treadmill exercise test was performed if the participant was judged to be able to walk on a treadmill at a rate of at least $2.5 \mathrm{mph}$ and if there was no ECG evidence of left ventricular hypertrophy, ventricular-paced rhythm, left bundle-branch block, or the Wolff-Parkinson-White syndrome. Otherwise a nuclear stress test or echocardiographic stress test was performed. Treadmill and nuclear stress studies were routinely available between 7 am and 10:30 pm, on both weekdays and weekends. All the results of the cardiac function studies were interpreted by staff cardiologists. The Duke treadmill scoring system was used to score the performance on the treadmill exercise test; a score of $\geq 5$ was considered negative. The results of imaging studies were classified as negative, equivocal, or positive. All the participants with negative results on a treadmill or imaging study were discharged to their homes. Participants with a treadmill score of $\leq 5$ or positive results on an imaging study were admitted to the hospital. All those who were discharged from the CPU returned to the outpatient clinic within $72 \mathrm{~h}$ for a follow-up evaluation with a staff cardiologist

Outcomes

Primary outcome: occurrence of nonfatal MI, death, acute congestive heart failure, stroke, or out-ofhospital cardiac arrest (composite outcome)

Secondary outcomes: additional visits to the ED for chest pain or the use of any of the following tests and procedures: cardiac revascularisation, cardiac diagnostic tests and any hospitalisation for cardiac care during the 6 months after randomisation, and use of resources

Notes Defined time limit of maximum stay in the SSU: not clearly defined

Duration of participation: 6 months

The long-term follow-up study can be found in Cullen 2011. We contacted trial authors to obtain further details of the trial, but no reply was received

Trial registration: NA

\section{Risk of bias}

\begin{tabular}{lll}
\hline Bias & Authors' judgement & Support for judgement \\
\hline $\begin{array}{l}\text { Random sequence genera- } \\
\text { tion (selection bias) }\end{array}$ & Unclear risk & $\begin{array}{l}\text { Participants were stratified according to sex, previous MIs, previous revascu- } \\
\text { larisation procedure, but random sequence generation not described }\end{array}$ \\
\hline $\begin{array}{l}\text { Allocation concealment } \\
\text { (selection bias) }\end{array}$ & Unclear risk & Allocation concealment not described \\
\hline $\begin{array}{l}\text { Baseline outcome mea- } \\
\text { surement }\end{array}$ & Low risk & Baseline outcome measurement not relevant \\
\hline Baseline characteristics & Low risk & $\begin{array}{l}\text { Baseline characteristics were described and similar in the intervention and } \\
\text { control group }\end{array}$ \\
\hline
\end{tabular}


Farkouh 1998 (Continued)

Blinding of outcome as- Unclear risk Blinding of outcome assessment was not described sessment (detection bias)

All outcomes

\begin{tabular}{lll}
\hline $\begin{array}{l}\text { Incomplete outcome data } \\
\text { (attrition bias) } \\
\text { All outcomes }\end{array}$ & Low risk & $\begin{array}{l}\text { Authors excluded } 17 \text { participants due to lack of consent to obtain surveillance } \\
\text { data (8 in the intervention group and } 9 \text { in the usual-care group) in the long- } \\
\text { term follow-up; clearly described and still < 15\% of included participants }\end{array}$ \\
\hline $\begin{array}{l}\text { Contamination } \\
\text { High risk }\end{array}$ & $\begin{array}{l}115 / 212 \text { participants in the intervention group were transferred to the cardiol- } \\
\text { ogy service (usual care) }\end{array}$ \\
\hline $\begin{array}{l}\text { Selective reporting (re- } \\
\text { porting bias) }\end{array}$ & High risk & $\begin{array}{l}\text { The trial was not registered a in trial register. No protocol was described. All } \\
\text { outcomes defined in the methods section were reported }\end{array}$ \\
\hline $\begin{array}{l}\text { Other bias } \\
\text { Low risk }\end{array}$ & $\begin{array}{l}\text { The trial appeared to be free of other components that could put it at risk of } \\
\text { bias }\end{array}$ \\
\hline $\begin{array}{l}\text { Overall risk of bias } \\
\text { All outcomes }\end{array}$ & High risk & $\begin{array}{l}\text { High risk of bias and/or unclear risk of bias in one or more domains: blinding of } \\
\text { outcome assessment, incomplete outcome data, or selective reporting }\end{array}$ \\
\hline
\end{tabular}

\section{Gomez 1996}

Methods Parallel randomised trial

Aim of trial: to compare the safety and cost-effectiveness of a Rapid Rule-Out of Myocardial Ischemia Observation (ROMIO)-protocol in an ED-based Chest Pain Evaluation Unit for ruling out ischaemia with a routine hospital care strategy

Setting: single-centre trial conducted in an urban, tertiary, non-profit, public university hospital, Salt Lake City, Utah, USA, no information on trial dates, trial was conducted over a period of 19 months

Number of nurses/bed: unclear

Number of physicians/bed: unclear

Type of SSU: specialised unit, part of a multipurpose ED

Participants Chest pain participants with low probability of $\mathrm{MI}$

Inclusion criteria: age $>30$ years, chest pain-correlated symptoms that could not be explained by local trauma or abnormalities on a chest X-ray film and was considered by the ED physician to be sufficiently suggestive of myocardial ischaemia to require hospital admission to rule out infarction or unstable angina, presentation suggesting a low $(<7 \%)$ predicted probability of infarction, and absence of acute ischaemic changes on baseline ECG

Exclusion criteria: ECG evidence of acute ischaemia, sustained ventricular tachycardia or non-sustained ventricular tachycardia, frequent ventricular ectopic activity or supraventricular tachycardia requiring iv medications, 2nd- or 3rd-degree heart block or new bundle branch block, need for iv nitroglycerin, systolic BP > $220 \mathrm{~mm} \mathrm{Hg}$ or diastolic pressure > $120 \mathrm{~mm} \mathrm{Hg}$ despite therapy, congestive heart failure requiring iv medications or intensive monitoring, other conditions requiring iv medications or intensive nursing care

Numbers recruited: 100,50 to the intervention group vs 50 to the usual-care group

Participants analysed for the primary outcome: 100

Mean age: 50 (SD not provided) in intervention group vs 53 (SD not provided) in usual-care group

Male: $31(62 \%)$ in intervention group vs $30(60 \%)$ in usual-care group 
Gomez 1996 (Continued)

Interventions
Intervention: placement in chest pain evaluation unit and rapid rule-out protocol defined by iv access, administration of $325 \mathrm{mg}$ oral aspirin, oxygen therapy if needed, serial creatine kinase and MB isoenzyme levels at 0, 3, 6 and $9 \mathrm{~h}$, continuous ST-segment monitoring was performed. If no signs of ischaemia was found, participants underwent symptom-limited graded exercise test. If sign of ischaemia was found, participants were transferred to a coronary care unit.

Usual care: routine care was admission to hospital, participants were managed by their attending physicians, who made all further triage, diagnostic and therapeutic decisions, including choice of assigned unit (coronary care unit, telemetry bed, general floor), laboratory testing, drug therapy, diagnostic testing and procedures, length of hospital stay, and timing of hospital discharge

Participants in both groups, who were unable to perform or complete an exercise test or whose test result was equivocal or not interpretable, underwent dobutamine stress echocardiography. Participants who had negative results on a treadmill test or dobutamine stress echocardiogram were discharged home.

Intervention in detail: participants were placed in the chest pain evaluation unit, where prewritten orders detailing the rapid rule-out protocol were followed, iv access was obtained (saline solution-filled catheter), and $325 \mathrm{mg}$ of oral aspirin was administered. Oxygen was given only for dyspnoea or for an oxygen saturation lower than $90 \%$ as assessed by pulse oximetry. Serial creatine kinase and MB isoenzyme levels were obtained at $0.3,6$ and $9 \mathrm{~h}$. Continuous ST-segment monitoring was performed. Participants who did not have ischaemic changes (with or without chest pain) on serial ECGs and had negative serial cardiac enzyme values negative for infarction underwent a symptom-limited graded exercise test. Testing was to be performed in a timely manner (generally 9-12 $\mathrm{h}$ after admission and between 7:00 am and 1:00 am). Participants were transferred from the chest pain evaluation unit to a monitored bed or the coronary care unit if they had 1) ECG changes consistent with acute ischaemia; 2) positive findings on creatine kinase MB determinations, rest echocardiogram, graded exercise or dobutamine stress echocardiographic tests; or 3) any other condition requiring hospital admission for further evaluation and treatment.
Primary outcome: length of stay in hospital, charges for initial stay, charges for 30 days of follow-up

Secondary outcomes: missed diagnosis of MI, postrandomisation hospital charges by category, frequency of making a final diagnosis of acute MI or unstable angina (overall and by trial group)

\section{Notes}

Defined time limit of maximum stay in the SSU: no time limit clearly described

Follow-up was performed at 30 days

Trial registration: no registration

None of the usual-care group participants were initially assigned to a bed in a coronary care unit bed, in contrast to the traditional approach at the time

Received additional information from trial authors by 2 March 2017

Information on re-visits for chest pain which led to in-hospital stress tests was available, but not clear whether authors recorded hospital readmissions for participants.

\section{Risk of bias}

\begin{tabular}{lll}
\hline Bias & Authors' judgement & Support for judgement \\
\hline $\begin{array}{l}\text { Random sequence genera- } \\
\text { tion (selection bias) }\end{array}$ & Unclear risk & Random sequence generation not described \\
\hline $\begin{array}{l}\text { Allocation concealment } \\
\text { (selection bias) }\end{array}$ & Low risk & Allocation concealment by sealed envelopes \\
\hline $\begin{array}{l}\text { Baseline outcome mea- } \\
\text { surement }\end{array}$ & Low risk & Baseline outcome measurement not relevant \\
\hline \hline
\end{tabular}


Gomez 1996 (Continued)

$\begin{array}{ll}\text { Baseline characteristics } \quad \text { Low risk } & \begin{array}{l}\text { Baseline characteristics were described and similar in the intervention group } \\ \text { and usual-care group }\end{array}\end{array}$
and usual-care group

Blinding of outcome as- Unclear risk

Unclear risk, not described in trial publication. Trial authors told us that those sessment (detection bias) documenting length of stay in hospital and hospital charges were not involved All outcomes in the protocol, but blinding was not clearly described. Other outcomes were assessed non-blinded (we did not assess these outcomes in the current review)

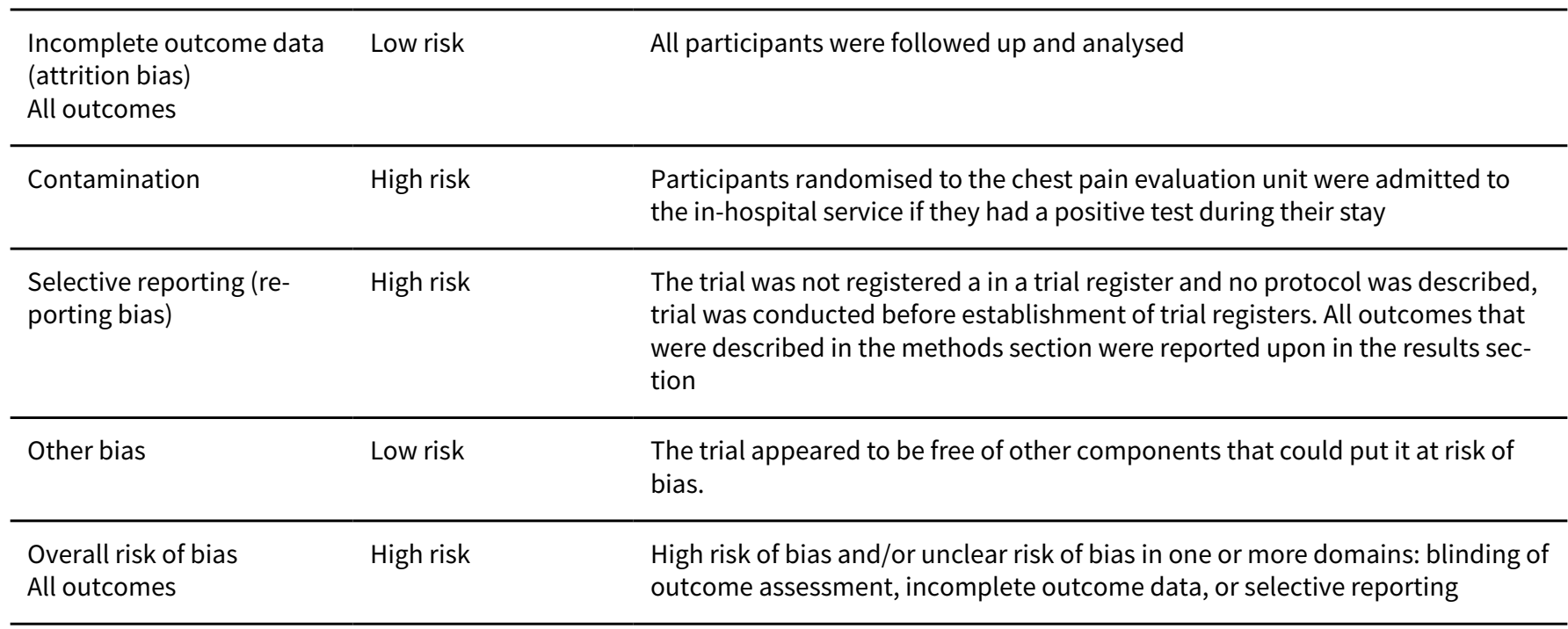

\section{McDermott 1997}

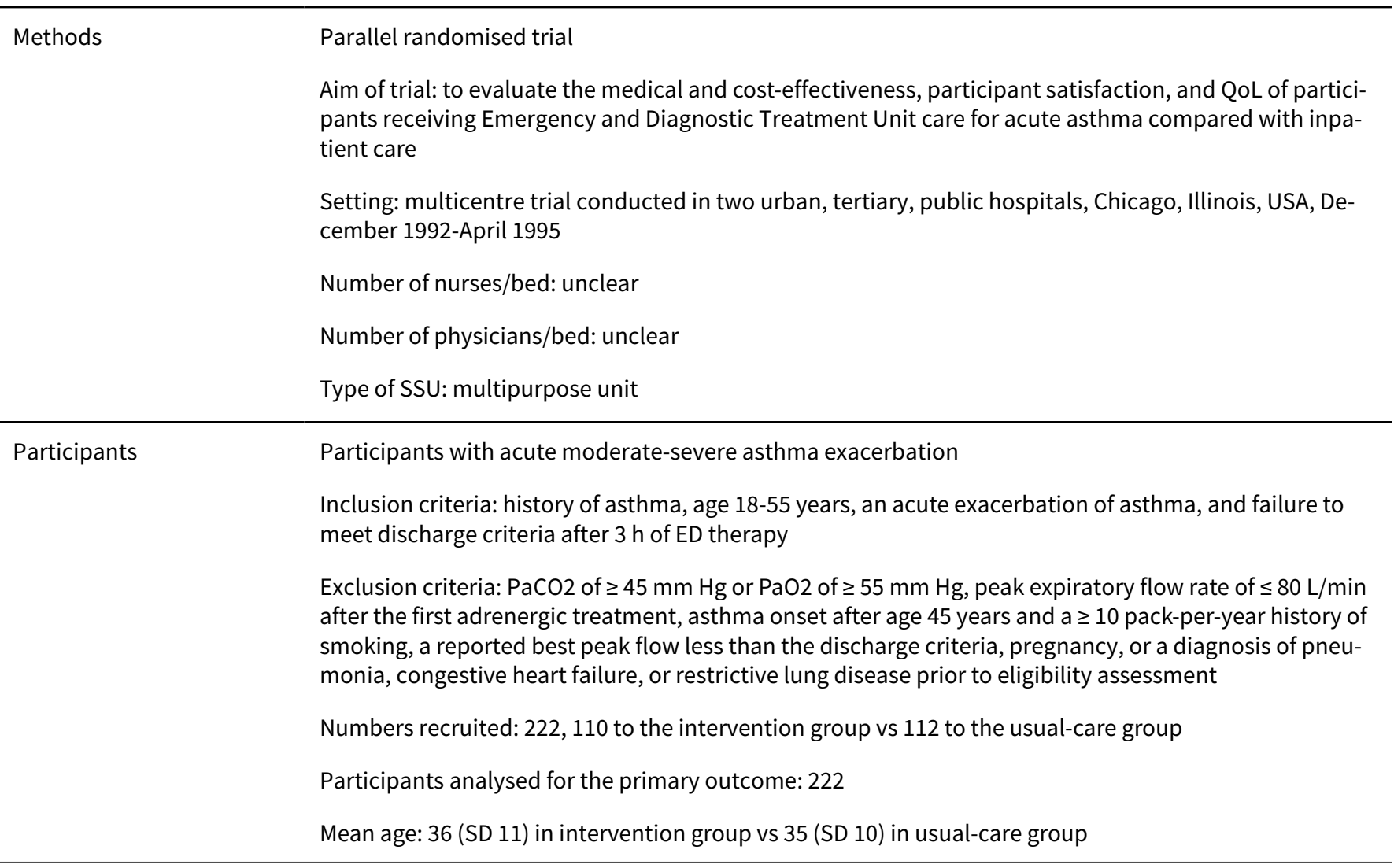


McDermott 1997 (Continued)

Male: 64 (58)\% in intervention group vs 70 (64)\% in usual-care group

Interventions Intervention: standardised treatment protocol in Emergency and Diagnostic Treatment Unit with scheduled administration of nebuliser at h: 4, 6, 8, 10, and 12 and a repeated steroid dose at hour: 6 . If predefined criteria were met (see below), participants were discharged. Discharge criteria were assessed repeatedly. If discharge criteria were not met within $12 \mathrm{~h}$, participants were admitted to in-hospital service

Usual care: treatment in a hospital ward according to national asthma guidelines i.e. handheld nebuliser every second hour for 3 treatments after admission and 4 times thereafter, and $60 \mathrm{mg}$ of methylprednisolone on arrival and every $6 \mathrm{~h}$ thereafter. Discharge criteria were similar to the intervention group, but participants were only assessed at time of arrival at ward and then on daily rounds

Discharge criteria for both groups: high-risk participants required a peak expiratory flow rate of $60 \%$ of the predicted value, while participants not at high risk needed a peak expiratory flow rate of $50 \%$. Participants were at high risk if the index ED visit was for a short-term relapse (second visit within 10 days) or if the participant could recall any of the following: previous intensive care unit admission or intubation, hospitalisation for asthma within the previous year, $\geq 3$ ED visits for asthma in the previous 6 months, or use of oral steroids for more than half of the previous year

Outcomes
No definition of outcome hierarchy
Outcomes: relapse rates, discharge rate, length of stay in hospital, minor morbidity (cough, wheez-
ing, dyspnoea, nocturnal awakenings), moderate morbidity (major lifestyle-limitating events e.g. days
missed from work or school, days incapacitated during waking hours), major morbidity (unscheduled
visits for treatment of acute asthma i.e. relapse), direct medical costs, participant satisfaction, and QoL

Notes

Defined time limit of maximum stay in the SSU: $12 \mathrm{~h}$

Follow-up: week 1, 3, 5, 7, 8 after initial attendance

Additional reports of the trial is found in Rydman 1998 and Rydman 1999

Trial registration: NA

We contacted trial authors twice but no reply received

\section{Risk of bias}

\begin{tabular}{|c|c|c|}
\hline Bias & Authors' judgement & Support for judgement \\
\hline $\begin{array}{l}\text { Random sequence genera- } \\
\text { tion (selection bias) }\end{array}$ & Unclear risk & Method of random sequence generation not described \\
\hline $\begin{array}{l}\text { Allocation concealment } \\
\text { (selection bias) }\end{array}$ & Unclear risk & Allocation concealment not described \\
\hline $\begin{array}{l}\text { Baseline outcome mea- } \\
\text { surement }\end{array}$ & Low risk & No difference in QoL measured at baseline \\
\hline Baseline characteristics & Low risk & Described and evenly distributed \\
\hline $\begin{array}{l}\text { Blinding of outcome as- } \\
\text { sessment (detection bias) } \\
\text { All outcomes }\end{array}$ & Low risk & Follow-up visits were performed by personnel blinded to the allocation \\
\hline $\begin{array}{l}\text { Incomplete outcome data } \\
\text { (attrition bias) } \\
\text { All outcomes }\end{array}$ & Unclear risk & $\begin{array}{l}\text { Dropout and withdrawals insufficiently described e.g. trial authors stated that } \\
65 / 110 \text { in the intervention group and 67/112 participants in the usual-care } \\
\text { group showed up at } 1 \text { week follow-up, but they only report QoL outcomes for }\end{array}$ \\
\hline
\end{tabular}



satisfaction, reason for dropouts were not described

\begin{tabular}{lll}
\hline Contamination & Unclear risk & Not described \\
\hline $\begin{array}{l}\text { Selective reporting (re- } \\
\text { porting bias) }\end{array}$ & High risk & $\begin{array}{l}\text { No trial registration. No protocol was described. All outcomes described in the } \\
\text { methods section were reported }\end{array}$ \\
\hline Other bias & Unclear risk & $\begin{array}{l}\text { Authors (Rydman, McDermott, Roberts, Zalenski, Murphy, McCarren, Kampe) } \\
\text { had written more than 3 papers on SSUs, academic bias may be present. The } \\
\text { trial appeared to be free of other components that could put it at risk of bias }\end{array}$ \\
\hline $\begin{array}{l}\text { Overall risk of bias } \\
\text { All outcomes }\end{array}$ & High risk & $\begin{array}{l}\text { High risk of bias and/or unclear risk of bias in one or more domains: blinding of } \\
\text { outcome assessment, incomplete outcome data, or selective reporting }\end{array}$ \\
\hline
\end{tabular}

\section{Miller 2010}

Parallel randomised trial
Aim of trial: to determine whether imaging with cardiac MRI in an observation unit would reduce med-
ical costs among participants with emergent non-low-risk chest pain who otherwise would be managed
with an inpatient care strategy
Setting: single-centre trial conducted in an urban, tertiary, public hospital, Winston-Salem, North Car-
olina, USA, January 2008-March 2009
Number of nurses/bed: unclear
Number of physicians/bed: unclear
Type of SSU: multipurpose unit (information provided by trial authors)

\section{Participants}

Chest pain with intermediate or high probability of ACS

Inclusion criteria: aged $\geq 18$ years, symptoms of possible ACS, care provider impression that inpatient evaluation was required, ability to be discharged if cardiac disease was excluded, and intermediate or high probability for experiencing ACS defined by either the ED care provider's clinical impression or a Thrombolysis in Myocardial Infarction risk score $\geq 2$

Exclusion criteria: initial increased troponin I level, new ST-segment elevation ( $\geq 1 \mathrm{mV}$ ) or depression ( $\geq$ $2 \mathrm{mV}$ ), inability to lie flat, systolic BP $<90 \mathrm{~mm} \mathrm{Hg}$, contraindications to MRI, refusal of follow-up procedures, terminal diagnosis with $<3$ months to live, pregnancy, renal insufficiency, chronic liver disease, or a history of heart, liver, or kidney transplant

Numbers recruited: 110,53 to the intervention group vs 57 to the usual-care group

Participants analysed for the primary outcome: 110

Median age (IQR): 55 (48-61) in intervention group vs 57 (47-64) in usual-care group

Male: 25 (47\%)\% in intervention group vs 30 (53\%) in usual-care group or physician assistants and supervised by a board-certified emergency physician. Protocol included cardiac biomarkers at 4 and $8 \mathrm{~h}$, and stress cardiac MRI examination available weekdays $8 \mathrm{am}-5 \mathrm{pm}$ If the 4 -h troponin I level was $<1.0 \mathrm{ng} / \mathrm{mL}$, participants could receive the stress cardiac MRI examination at the first available period.

Usual care: inpatient care i.e. evaluation by a consulting physician in the ED for the intent of admission, following usual procedures. Participants with established cardiology care or higher-risk profiles 
Miller 2010 (Continued)

were generally admitted to the cardiology service. Others were admitted to hospital-based services and cared for by internists or family medicine physicians. Care patterns in this group were determined by the care providers unaffected by the trial protocol. Cardiac MRI was available to these participants.

$\begin{array}{ll}\text { Outcomes } & \text { Primary outcome: direct medical cost of the index hospital visit } \\ & \text { Secondary outcome: in-trial registration reported to be correct admission decision (according to ACS } \\ \text { diagnosis within } 30 \text { days), number of participants randomised to intervention that were able to com- } \\ \text { plete cardiac MRI, utilisation of healthcare procedures, adverse events during MRI, adverse events lead- } \\ \text { ing to early termination of MRI }\end{array}$

\begin{tabular}{|c|c|}
\hline \multirow[t]{5}{*}{ Notes } & $\begin{array}{l}\text { Defined time limit of maximum stay in the SSU: } 24 \mathrm{~h} \text { but not hard cut-off (information provided by trial } \\
\text { authors) }\end{array}$ \\
\hline & Follow-up: 30 days \\
\hline & Long-term outcomes are reported in a secondary publication (Miller 2011) \\
\hline & Trial registration: nCT00678639 \\
\hline & Received additional information from trial authors by 28 February 2017 \\
\hline
\end{tabular}

\section{Risk of bias}

Bias Authors' judgement Support for judgement

Random sequence genera- Low risk tion (selection bias)

Computer-generated random numbers. Stratified block randomisation scheme, stratification according to presence of coronary artery disease and time of presentation (day or evening/night) (additional information provided by trial authors, did not change bias evaluation)

Allocation concealment $\quad$ Low risk $\quad$ Opaque, sealed envelopes
(selection bias)

Baseline outcome mea- Low risk $\quad$ Baseline outcome measurement not relevant
surement

\begin{tabular}{lll}
\hline Baseline characteristics & High risk & More participants in usual-care group had previous cardiovascular events \\
\hline $\begin{array}{l}\text { Blinding of outcome as- } \\
\text { sessment (detection bias) }\end{array}$ & High risk & No blinding (information provided by trial authors) \\
All outcomes & \\
\hline
\end{tabular}

\begin{tabular}{|c|c|c|}
\hline $\begin{array}{l}\text { Incomplete outcome data } \\
\text { (attrition bias) } \\
\text { All outcomes }\end{array}$ & Low risk & $\begin{array}{l}\text { Dropouts clearly described and below } 15 \% \text { of recruited participants ( } 2 \text { in inter- } \\
\text { vention group, } 0 \text { in usual-care group) }\end{array}$ \\
\hline
\end{tabular}

\begin{tabular}{lll}
\hline Contamination & High risk & Participants were transferred to inpatient care from SSU if needed \\
\hline $\begin{array}{l}\text { Selective reporting (re- } \\
\text { porting bias) }\end{array}$ & Low risk & All outcomes stated in trial registration reported \\
\hline Other bias & High risk & $\begin{array}{l}\text { Trial authors (Miller, Hwang, Case, Hoekestra, Harper, Lefebvre)) had written } \\
\text { more than 3 papers on SSUs, academic bias may be present. Dr. Miller had re- } \\
\text { ceived support from Siemens, the MRI scanner used was a Siemens model. }\end{array}$ \\
\hline $\begin{array}{l}\text { Overall risk of bias } \\
\text { All outcomes }\end{array}$ & High risk & $\begin{array}{l}\text { High risk of bias and/or unclear risk of bias in 1 or more domains: blinding of } \\
\text { outcome assessment, incomplete outcome data, or selective reporting }\end{array}$ \\
\hline
\end{tabular}


Miller 2013

Parallel randomised trial
Aim of trial: to determine whether imaging with cardiac MRI in an observation unit would reduce med-
ical costs among participants with emergent non-low risk chest pain who otherwise would be managed
with an inpatient care strategy
Setting: single-centre trial conducted in an urban, tertiary, public hospital, Winston-Salem, North Car-
olina, USA, January 2010-October 2011
Number of nurses/bed: unclear
Number of physicians/bed: unclear
Type of SSU: multipurpose unit (information provided by trial authors)

Participants

Participants with chest pain and intermediate or high probability of ACS

Inclusion criteria: $\geq 21$ years old presenting with symptoms suggestive of ACS, intermediate risk chest pain defined as either a Thrombolysis in Myocardial Infarction risk score of $\geq 2$ or a physician global risk assessment based on the ACC/AHA guidelines of intermediate or high risk. At time of enrolment, the ED attending had to declare the participant as being safe for observation unit care, and that the participant could be discharged home if cardiac disease was excluded as the cause of symptoms

Exclusion criteria: definite ACS at the time of enrolment, known inducible ischaemia, hypotension, contraindications to cardiac MRI, life expectancy $<3$ months, pregnancy, coronary revascularisation within 6 months, and increased risk for nephrogenic systemic fibrosis, clinical concern for acute kidney injury, hepatorenal syndrome, or solid organ transplant

Numbers recruited: 105,52 to the intervention group vs 53 to the usual-care group

Participants analysed for the primary outcome: 105

Median age (IQR): 54 (35-91) in intervention group vs 59 (40-76) in usual-care group

Male: 28 (54\%) in intervention group vs 29 (55)\% in usual-care group

Interventions

Intervention: standardised protocol in ED observation unit, the unit was staffed by nurse practitioners or physician assistants and supervised by a board-certified emergency physician. Protocol included cardiac biomarkers at $4 \mathrm{~h}$ and $8 \mathrm{~h}$, and vasodilator cardiac MRI examination available weekdays 8 am- 5 $\mathrm{pm}$ If the 4-h troponin I level was $<1.0 \mathrm{ng} / \mathrm{mL}$, participants could receive the stress cardiac MRI examination at the first available period. Interpretations, the need for cardiology consultation, and decisions to perform revascularisation were not directed by the trial protocol. The intervention protocol differed from Miller 2010 in the cardiac MRI sequences in the intervention arm.

Usual care: usual-care participants underwent consultation in the ED by the admitting service in accordance with customary practice (Cardiology, Internal Medicine, and Family medicine services) and could be admitted to any service. Care delivery was not dictated by a trial protocol.

Outcomes Primary outcome: coronary revascularisation, all-cause hospital readmission, or recurrent cardiac testing within 90 days of randomisation (composite outcome)

Secondary outcomes: index visit length of stay in hospital, safety events (all-cause mortality within 90 days, adverse events related to index visit stress testing, ACS after discharge and within 90 days of randomisation)

Notes Defined time limit of maximum stay in the SSU: $24 \mathrm{~h}$ but not hard cut-off (information provided by trial authors)

Follow-up: 90 days 
Miller 2013 (Continued)

Trial planned to recruit 146 participants, but the number of events defining the sample size were obtained after enrolment of 105 participants and trial was stopped

Trial registration: NCT01035047

Received additional information from trial authors by 28 February 2017

\section{Risk of bias}

\begin{tabular}{|c|c|c|}
\hline Bias & Authors' judgement & Support for judgement \\
\hline $\begin{array}{l}\text { Random sequence genera- } \\
\text { tion (selection bias) }\end{array}$ & Low risk & $\begin{array}{l}\text { Computer-generated random numbers, variable sized permuted blocks, strati- } \\
\text { fied by presence of known coronary disease }\end{array}$ \\
\hline $\begin{array}{l}\text { Allocation concealment } \\
\text { (selection bias) }\end{array}$ & Low risk & Concealed in computer system \\
\hline $\begin{array}{l}\text { Baseline outcome mea- } \\
\text { surement }\end{array}$ & Low risk & Baseline outcome measurement not relevant \\
\hline Baseline characteristics & Low risk & Described and evenly distributed \\
\hline $\begin{array}{l}\text { Blinding of outcome as- } \\
\text { sessment (detection bias) } \\
\text { All outcomes }\end{array}$ & High risk & No blinding (information provided by trial authors) \\
\hline $\begin{array}{l}\text { Incomplete outcome data } \\
\text { (attrition bias) } \\
\text { All outcomes }\end{array}$ & High risk & $\begin{array}{l}\text { Trial planned to recruit } 146 \text { participants, but the number of events defining the } \\
\text { sample size were obtained after enrolment of } 105 \text { participants and trial was } \\
\text { stopped. } \\
\text { No dropouts. Participants lost to follow-up were censored at the point of last } \\
\text { contact. They were clearly described, the number was below } 15 \% \text { of trial pop- } \\
\text { ulation }(6 / 105)\end{array}$ \\
\hline Contamination & High risk & Participants were transferred to inpatient care from SSU if needed \\
\hline $\begin{array}{l}\text { Selective reporting (re- } \\
\text { porting bias) }\end{array}$ & Low risk & All outcomes stated in trial registration reported upon \\
\hline Other bias & High risk & $\begin{array}{l}\text { Dr. Miller had received support from Siemens, the MRI scanner used was a } \\
\text { Siemens model. }\end{array}$ \\
\hline $\begin{array}{l}\text { Overall risk of bias } \\
\text { All outcomes }\end{array}$ & High risk & $\begin{array}{l}\text { High risk of bias and/or unclear risk of bias in } 1 \text { or more domains: blinding of } \\
\text { outcome assessment, incomplete outcome data, or selective reporting }\end{array}$ \\
\hline
\end{tabular}

\section{Roberts 1997}

Parallel randomised trial
Aim of trial: the objectives of this trial were to evaluate the hospital admission rate, cost, and length
of stay in hospital using an accelerated diagnostic protocol in a chest pain observation unit compared
with hospitalised controls
Setting: single-centre trial at an urban, tertiary, public hospital, Chicago, Illinois, USA, January 1993-
April 1995
Number of nurses/bed: unclear


Roberts 1997 (Continued)

Number of physicians/bed: unclear

Type of SSU: specialised unit

Participants
Inclusion criteria: age $>20$ years, hospitalisation necessary (physician judgement), low probability for
acute MI (Goldman algorithm), ability to perform ECG exercise stress test
Exclusion criteria: history of prehospital or ED complication of acute ischaemia or MI, new ECG findings
consistent with MI or ischaemia, protocol performance put participant at risk, concurrent or alternate
noncardiac diagnosis requiring urgent hospitalisation, problem with performance or interpretation of
ECG exercise stress test

Numbers recruited: 165,82 to the intervention group vs 83 to the usual-care group

Participants analysed for the primary outcome: 165

Mean age: 47.3 (SD 9.9) in intervention group vs 48.0 (SD 11.4) in usual-care group

Male: 45 (54.9\%) in intervention group vs 44 (53)\% in usual-care group

Interventions

Intervention: accelerated diagnostic protocol in a chest pain observation unit consisting of 12-h rhythm monitoring, creatine kinase-MB levels at $0,4,8$, and $12 \mathrm{~h}$, ECGs at 0,6 , and $12 \mathrm{~h}$, clinical examination and review of test results by an attending physician at 0,6 , and $12 \mathrm{~h}$ or for any change in condition, aspirin, $2 \mathrm{~L}$ oxygen by nasal cannula, and iv line, nitrates were given if recurrent chest pain. If all the clinical and test findings were negative, participants were taken to the adjacent cardiac laboratory for immediate ECG exercise stress test, if negative, participants were discharged. If positive or uncertain test results at any time, participants were admitted to in-hospital service

Usual care: participants were admitted to the telemetry unit on the internal medicine service for standard management at the time ( 3 sets of cardiac enzyme studies, two ECGs, and $24 \mathrm{~h}$ of cardiac and clinical monitoring), management was at the discretion of the internal medicine attending physician

Outcomes Not outcome hierarchy, but sample size calculation was based on costs.

Outcomes: length of stay in hospital, costs, hospital admission rate

Notes Sample size was estimated to 200 participants, only 166 participants were randomised

When participants in the intervention group reached predefined discharge criteria, they were transferred to another ward for additional $24 \mathrm{~h}$ of monitoring, because this trial was part of a larger trial investigating diagnostic accuracy

Defined time limit of maximum stay in the SSU: $12 \mathrm{~h}$

Follow-up: 8 weeks

Trial registration: NA

We contacted trial authors twice, but no response received

\section{Risk of bias}

\begin{tabular}{lll}
\hline Bias & Authors' judgement & Support for judgement \\
\hline $\begin{array}{l}\text { Random sequence genera- } \\
\text { tion (selection bias) }\end{array}$ & Low risk & Computer-generated random numbers \\
\hline $\begin{array}{l}\text { Allocation concealment } \\
\text { (selection bias) }\end{array}$ & Low risk & $\begin{array}{l}\text { Opaque enveloped constructed by trial personnel not connected to the ED or } \\
\text { enrolment process }\end{array}$ \\
\hline
\end{tabular}


Roberts 1997 (Continued)

Baseline outcome mea- Low risk Baseline outcome measurement not relevant surement

\begin{tabular}{lll}
\hline Baseline characteristics & Low risk & Described and evenly distributed \\
\hline $\begin{array}{l}\text { Blinding of outcome as- } \\
\text { sessment (detection bias) }\end{array}$ & Unclear risk & $\begin{array}{l}\text { Cost and length of stay in hospital were abstracted from research forms, med- } \\
\text { ical records, and hospital information systems. Uncertain whether assessors } \\
\text { All outcomes }\end{array}$ \\
\hline
\end{tabular}

\begin{tabular}{ll}
\hline $\begin{array}{l}\text { Incomplete outcome data } \\
\text { (attrition bias) }\end{array}$ & Low risk \\
All outcomes & was low $(<15 \%)$
\end{tabular}

\begin{tabular}{lll}
\hline Contamination & High risk & Participants were transferred to inpatient care from SSU if needed \\
\hline $\begin{array}{l}\text { Selective reporting (re- } \\
\text { porting bias) }\end{array}$ & High risk & $\begin{array}{l}\text { No trial registration. No protocol was described. Trial authors described out- } \\
\text { comes as defined in the methods section }\end{array}$ \\
\hline Other bias & Unclear risk & $\begin{array}{l}\text { Authors (Zalenski) had written more than 3 papers on SSUs, academic bias } \\
\text { may be present. }\end{array}$ \\
$\begin{array}{l}\text { The trial appeared to be free of other components that could put it at risk of } \\
\text { bias }\end{array}$ \\
$\begin{array}{l}\text { Overall risk of bias } \\
\text { All outcomes }\end{array}$ & High risk & $\begin{array}{l}\text { High risk of bias and/or unclear risk of bias in 1 or more domains: blinding of } \\
\text { outcome assessment, incomplete outcome data, or selective reporting }\end{array}$ \\
\hline
\end{tabular}

Ross 2007

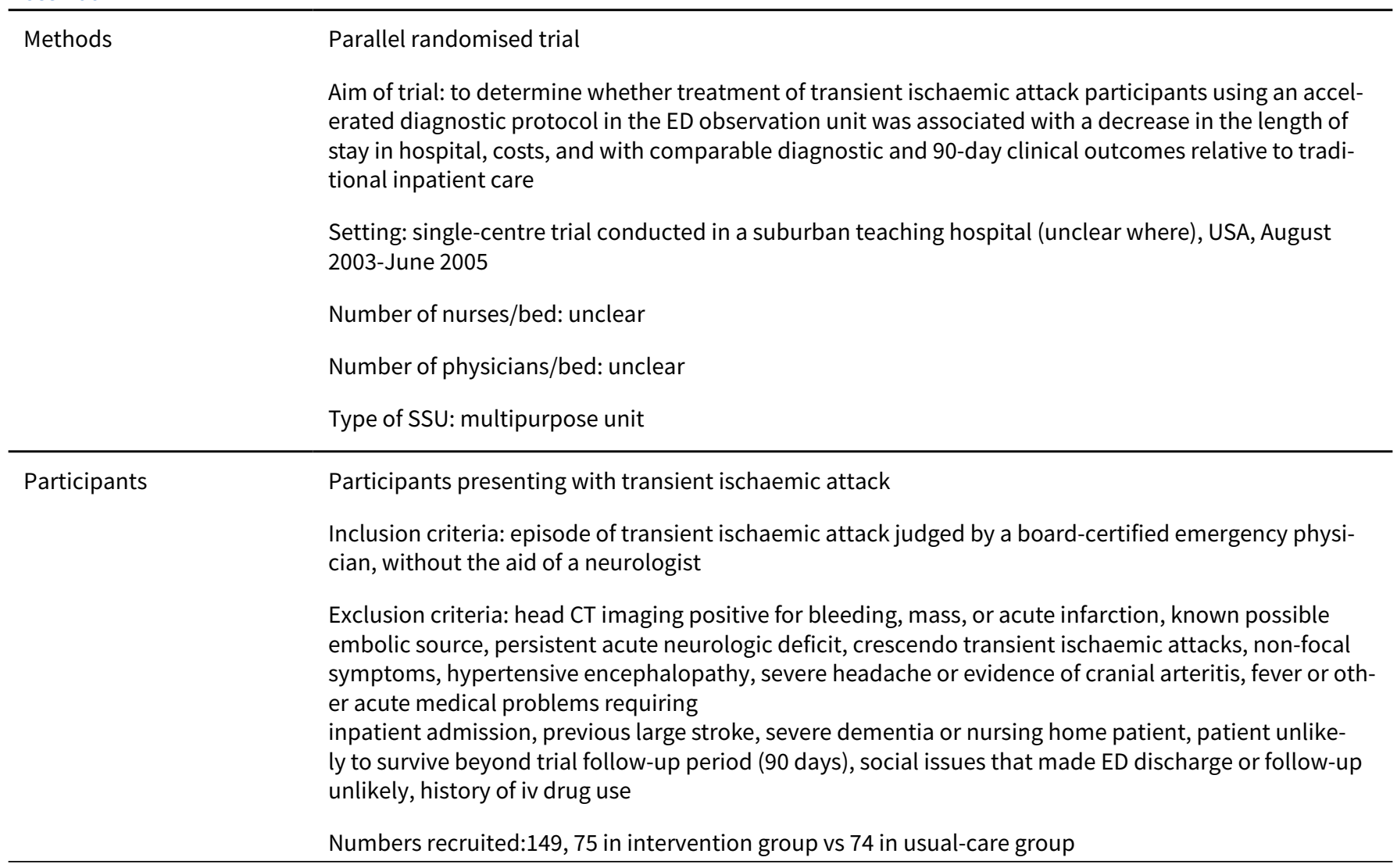


Ross 2007 (Continued)

Participants analysed for the primary outcome: 149

Mean age: 68.4 (SD 15.3) in intervention group vs 67.7 (SD 15.4) in usual-care group

Male: 31 (41\%) in intervention group vs (53)\% in usual-care group

Interventions

Intervention: diagnostic protocol in ED observation unit consisting of carotid imaging (Doppler, magnetic resonance angiography), echocardiography, serial evaluations (serial assessments by nurses, emergency physicians, and physician assistants, and a neurologist consultation), cardiac monitoring for at least $12 \mathrm{~h}$. Participants were admitted to inward service if they had recurrent neurologic symptoms or developed stroke, significant carotid stenosis requiring urgent revascularisation, evidence of a thromboembolic source, requiring inpatient anticoagulation treatment with heparin, unable to complete the evaluation or be safely discharged home in $18-24 \mathrm{~h}$, or if the physician thought that admission was needed. Participants who were discharged from the ED observation unit were instructed to follow up within 1-3 days with their primary care physician or neurologist.

Usual care: hospital admission, could be both stroke unit, internal medicine or other. Participants admitted to the inpatient control group had their primary attending physician or hospitalist service attending physician contacted to discuss the participants' admission. From this discussion, the emergency physician completed the enclosed inpatient admission order forms, and the participant was admitted to that attending physician, with most admissions being to the internal medicine service. The hospital had a designated stroke unit; however, a bed in that unit was not routinely available for transient ischaemic attack patients. Alternatively, patients were admitted to an available regular medical floor and wore a portable cardiac-monitoring device. The decision to cancel or modify the initial admitting orders and when to discharge the participant home was made by the admitting attending physician on a case-by-case basis

Primary outcome: index visit length of stay in hospital
Secondary outcomes: 90 -day total direct cost and clinical
outcomes, which included stroke, major clinical events,
recidivism, the timeliness of diagnostic testing, the percentage of tests completed, and test outcomes

Notes

Defined time limit of maximum stay in the SSU: $24 \mathrm{~h}$

Follow-up: 8 weeks

Trial registration: NA

We contacted trial authors twice for additional information but no response received

\section{Risk of bias}

\begin{tabular}{lll}
\hline Bias & Authors' judgement & Support for judgement \\
\hline $\begin{array}{l}\text { Random sequence genera- } \\
\text { tion (selection bias) }\end{array}$ & Low risk & Computer-generated random numbers \\
\hline $\begin{array}{l}\text { Allocation concealment } \\
\text { (selection bias) }\end{array}$ & Low risk & Sealed envelopes \\
\hline $\begin{array}{l}\text { Baseline outcome mea- } \\
\text { surement }\end{array}$ & Low risk & Baseline outcome measurement not relevant \\
\hline $\begin{array}{l}\text { Baseline characteristics } \\
\text { Blinding of outcome as- } \\
\text { sessment (detection bias) } \\
\begin{array}{l}\text { All outcomes } \\
\hline\end{array}\end{array}$ & Low risk risk & Described and evenly distributed \\
\hline
\end{tabular}


Ross 2007 (Continued)
Incomplete outcome data
(attrition bias)
Low risk All participants were followed up
All outcomes

\begin{tabular}{lll}
\hline Contamination & High risk & Participants were transferred to inpatient care from SSU if needed \\
\hline $\begin{array}{l}\text { Selective reporting (re- } \\
\text { porting bias) }\end{array}$ & High risk & $\begin{array}{l}\text { No trial registration. No protocol was described. All outcomes described in the } \\
\text { method section were reported in the paper. }\end{array}$ \\
\hline Other bias & Unclear risk & $\begin{array}{l}\text { The trial appeared to be free of other components that could put it at risk of } \\
\text { bias. }\end{array}$ \\
\hline $\begin{array}{l}\text { Overall risk of bias } \\
\text { All outcomes }\end{array}$ & High risk & $\begin{array}{l}\text { High risk of bias and/or unclear risk of bias in } 1 \text { or more domains: blinding of } \\
\text { outcome assessment, incomplete outcome data, or selective reporting }\end{array}$ \\
\hline
\end{tabular}

\section{Rydman 1997}

Methods
$\begin{aligned} & \text { Parallel randomised trial } \\ & \text { observation unit and those who underwent hospital inpatient observation }\end{aligned}$
Setting: single-centre trial at an urban, tertiary, public hospital, Chicago, Illinois, USA, trial dates not
defined
Number of nurses/bed: unclear
Number of physicians/bed: unclear
Type of SSU: specialised unit

\section{Participants}

Chest pain patients with low risk of acute MI

Inclusion criteria: participants in need of hospital admission but at low probability (by a validated algorithm according to trial authors) for acute MI

Exclusion criteria: known coronary artery disease, cardiac complications, severe comorbidities, or inability to perform exercise testing

Numbers recruited: 104, 52 to the intervention group vs 52 to the usual-care group

Participants analysed for the primary outcome: 104

Mean age: 47.9 (SD 8.6) in intervention group vs 47.3 (SD 12.0) in usual-care group

Male: $61 \%$ in intervention group vs $59 \%$ in usual-care group

\section{Interventions}

Intervention: transfer to bed chest pain observation unit and 12-h chest pain protocol with serial measurements of creatine kinase-MB, ECGs, and clinical assessments followed by ECGs

Usual care: in-hospital observation

\begin{tabular}{ll}
\hline Outcomes & Primary outcome: patient satisfaction \\
& $\begin{array}{l}\text { Secondary outcome: correlation of patient characteristics and process of care with overall patient sat- } \\
\text { isfaction }\end{array}$
\end{tabular}

Notes

Defined time limit of maximum stay in the SSU: $12 \mathrm{~h}$ 
Rydman 1997 (Continued)

Duration of participation: time from randomisation to either hospital discharge for inpatients and the end of diagnostic protocol for intervention group (thus, not same time points for groups)

The participants in the intervention group scored higher than those randomised to the inpatient hospitalisation protocol on 4 summary ratings of patient satisfaction measures: quality of the service, recommendation of the service to others, effective treatment of health problem, and overall satisfaction.

Trial registration: NA

We contacted trial authors twice for additional information but no response received

\section{Risk of bias}

\begin{tabular}{|c|c|c|}
\hline Bias & Authors' judgement & Support for judgement \\
\hline $\begin{array}{l}\text { Random sequence genera- } \\
\text { tion (selection bias) }\end{array}$ & Unclear risk & Method of random sequence generation not described \\
\hline $\begin{array}{l}\text { Allocation concealment } \\
\text { (selection bias) }\end{array}$ & Unclear risk & Allocation concealment not described \\
\hline $\begin{array}{l}\text { Baseline outcome mea- } \\
\text { surement }\end{array}$ & Low risk & Baseline outcome measurement not relevant \\
\hline Baseline characteristics & Low risk & Described and evenly distributed \\
\hline $\begin{array}{l}\text { Blinding of outcome as- } \\
\text { sessment (detection bias) } \\
\text { All outcomes }\end{array}$ & Unclear risk & $\begin{array}{l}\text { Assessments were performed by trained researcher, unclear whether they } \\
\text { were blinded to the allocation }\end{array}$ \\
\hline $\begin{array}{l}\text { Incomplete outcome data } \\
\text { (attrition bias) } \\
\text { All outcomes }\end{array}$ & Low risk & All participants followed up \\
\hline Contamination & Unclear risk & Not described \\
\hline $\begin{array}{l}\text { Selective reporting (re- } \\
\text { porting bias) }\end{array}$ & High risk & $\begin{array}{l}\text { No trial registration. No protocol was described. All outcomes described in the } \\
\text { methods section were reported. }\end{array}$ \\
\hline Other bias & Low risk & $\begin{array}{l}\text { The trial appeared to be free of other components that could put it at risk of } \\
\text { bias. }\end{array}$ \\
\hline $\begin{array}{l}\text { Overall risk of bias } \\
\text { All outcomes }\end{array}$ & High risk & $\begin{array}{l}\text { High risk of bias and/or unclear risk of bias in } 1 \text { or more domains: blinding of } \\
\text { outcome assessment, incomplete outcome data, or selective reporting }\end{array}$ \\
\hline
\end{tabular}

Shen 2004

Methods
Parallel randomised trial
Aim of trial: to assess whether an area designated for syncope evaluation in the ED observational unit
("syncope unit”) could affect diagnostic yield and the rate of hospital admission for syncope partici-
pants with intermediate-risk profiles for a poor prognosis
Setting: single-centre trial at a tertiary, private, not-for profit hospital, Rochester, Minnesota, USA, Jan-
uary 2000-April 2004

Number of nurses/bed: unclear 
Shen 2004 (Continued)

Number of physicians/bed: unclear

Type of SSU: specialised unit

Participants

Participants with syncope and an intermediate risk for an adverse cardiovascular outcome

Inclusion criteria: age $\geq 18$ years, living in Olmsted county and the surrounding 14 counties, syncope of undetermined cause, intermediate risk for an adverse cardiovascular outcome

Exclusion criteria: identified cause of syncope during initial evaluation in the ED, any condition that would require hospital admission, non-syncope syndromes (light-headedness, dizziness, vertigo, presyncope, coma, shock, spells, fall, metabolic syndrome, typical seizure presentation, or recurrence of known seizure, or other state of altered mentation, or cardiac arrest)

Numbers recruited: 103, 51 in intervention group vs 52 in usual-care group

Participants analysed for the primary outcome: 103

Mean age: 64 (SD 17) in intervention group vs 65 (SD 17) in usual-care group

Male: 25 (49)\% in intervention group vs 25 (48)\% in usual-care group

Interventions Intervention: standardised protocol in ED syncope unit. Protocol consisted of continuous cardiac monitoring in a designated area in the observational unit for up to $6 \mathrm{~h}$, hourly vital signs and orthostatic $\mathrm{BP}$, echocardiography if participants had abnormal cardiovascular examination findings or an abnormal ECG, tilt-table testing in an electrophysiological laboratory near the syncope unit (tilt table testing was only for selected participants). An ED physician and a registered nurse staffed the syncope unit. An electrophysiological consultation was obtained while the participant was in the syncope unit when interpretation of the tilt-table test result or triaging recommendations were needed. If these tests and consultations could not be performed while the participant was in the syncope unit, arrangements for an outpatient consultation at a Heart Rhythm Centre, tilt-table testing, or echocardiography could be made within $72 \mathrm{~h}$ after dismissal from the syncope unit. An educational booklet on syncope was given to each participant at the time of dismissal from the syncope unit. The collaborative effort of physician and nursing staff from the ED, cardiovascular diseases, and electrophysiology constituted the multidisciplinary approach in the syncope unit.

Usual care: usual care, the ED physician was responsible for making the decision whether further evaluation was required and the setting in which the evaluation should occur. Additional ED diagnostic testing was performed at the discretion of the ED physician on the basis of the participant's history, physical examination, and laboratory findings.

Outcomes Primary outcome: diagnostic yield (presumptive cause of syncope established) and hospital admission rate

Secondary outcomes: net diagnostic yield, length of hospital stay at the completion of the evaluation of the index event, all-cause mortality, and recurrent syncope during follow-up

Notes Defined time limit of maximum stay in the SSU: $6 \mathrm{~h}$

Follow-up: 2 years

Trial was stopped early because of poor recruitment.

Trial registration: no registration (trial was conducted from 2000-2004)

Received additional information from trial authors by 28 February 2017

\section{Risk of bias}


Shen 2004 (Continued)

Random sequence genera- Low risk $\quad$ Computer-generated allocation list
tion (selection bias)

tion (selection bias)

\begin{tabular}{ll}
\hline $\begin{array}{l}\text { Allocation concealment } \\
\text { (selection bias) }\end{array}$ & Low risk
\end{tabular}

Baseline outcome mea- Low risk
surement

\begin{tabular}{lll}
\hline Baseline characteristics & Low risk & Described and evenly distributed \\
\hline $\begin{array}{l}\text { Blinding of outcome as- } \\
\text { sessment (detection bias) }\end{array}$ & High risk & No blinding of outcome assessors (information by trial authors) \\
All outcomes &
\end{tabular}

\begin{tabular}{lll}
\hline $\begin{array}{l}\text { Incomplete outcome data } \\
\text { (attrition bias) } \\
\text { All outcomes }\end{array}$ & Unclear risk & Trial was stopped early due to poor recruitment \\
\hline Contamination & High risk & Participants were transferred to inpatient care from SSU if needed \\
\hline $\begin{array}{l}\text { Selective reporting (re- } \\
\text { porting bias) }\end{array}$ & High risk & $\begin{array}{l}\text { No trial registration (trial conducted 2000-2004). No protocol was described. } \\
\text { Trial authors have described outcomes as defined in the methods section }\end{array}$ \\
\hline $\begin{array}{l}\text { Other bias } \\
\text { Low risk }\end{array}$ & $\begin{array}{l}\text { The trial appeared to be free of other components that could put it at risk of } \\
\text { bias. }\end{array}$ \\
\hline $\begin{array}{l}\text { Oll outcomes } \\
\text { High risk }\end{array}$ & $\begin{array}{l}\text { High risk of bias and/or unclear risk of bias in 1 or more domains: blinding of } \\
\text { outcome assessment, incomplete outcome data, or selective reporting }\end{array}$ \\
\hline
\end{tabular}

\section{Strøm 2017a}

Methods
Parallel randomised trial
Aim of trial: to compare SSU hospitalisation with usual care at an Internal Medicine Department (IMD)
Setting: single-centre trial at a secondary, public, teaching hospital, Holbaek, Denmark, January 2015-
October 2016
Number of nurses/bed (per daytime/evening/night shifts): $3 / 2-4 / 1-2$ per 16 beds in intervention group
vs 6/3-4/2-3 per 20 beds in usual-care group
Number of physicians/bed (per daytime/evening/night shifts): $3 / 5$ available from ED/4 available from
ED per 16 beds in intervention group vs $4 / 2-3$ on-call in-house/2 on-call in-house per 20 beds in usu-
al-care group
Type of SSU: multipurpose unit

Participants
Participants with any type of internal medicine disease or condition
Inclusion criteria: age $\geq 75$ years, acutely admitted for an internal medicine disease, and assessed by an ED-physician to be suitable for SSU hospitalisation, green-tag triage (by the Danish Emergency Process Triage)
Exclusion criteria: previous participation in the trial, active participation in another clinical trial, lack of Danish civil registration number, residency in another country than Denmark, need of help getting to


the toilet in daily life, no awareness of the current date, time and location, no awareness of name and date of birth, no space in the SSU, or if informed consent could not be obtained

Numbers recruited: 430, 215 in intervention group vs 215 in usual-care group

Participants analysed for the primary outcome: 412

Mean age (IQR): 81 (IQR 76-86) in intervention group vs 82 (IQR 78-86) in usual-care group

Male: 97 (47\%) in intervention group vs 87 (41\%) in usual-care group

$\mathrm{CCl}(0 / 1 / 2 / 3 / 4 / 5$ or more, $\mathrm{n})=15 / 26 / 21 / 17 / 11 / 10$ in intervention group vs $20 / 27 / 26 / 13 / 9 / 5$ in usual-care group

Interventions

Intervention: placement in ED SSU. Discharge planning was initiated immediately after admission to the SSU. If the participant needed further diagnostic tests these were performed on the same terms as in the ED, including point-of-care ultrasonography available around the clock, acute blood samples analysed in the ED's point-of-care laboratory from 8 am-10 pm, and simple X-rays in the ED's X-ray room manned from 10 am-6 pm More advanced diagnostic examinations; such as CT or MRI scans were performed at the department of radiology on a fast-track basis. Participants were encouraged to mobilise as much as possible without assistance during the stay, which usually included getting minimal help to basic self-care activities such as bathing, getting out of bed, or walking around the SSU

Usual care: placement in internal medicine department ward, no standardised treatment protocols were applied

Outcomes Primary outcome: 90-day all-cause mortality

Secondary outcomes: mortality rate at conclusion of the trial, in-hospital mortality, adverse events during hospitalisation, change in Lawton IADL-score within 90 days from admission, length of stay in hospital, unplanned hospital readmissions within 30 days after discharge, relocation to a living facility with higher level of care within 90 days from admission, and transfer to another treatment facility during hospitalisation

Notes Defined time limit of maximum stay in the SSU: $72 \mathrm{~h}$ (not hard cut off, if deemed in participant's best interest they were allowed to stay longer)

Follow-up: minimum 90 days

Trial registration: NCT02395718

Strøm is first author of the current Cochrane Review, the trial paper was assessed by JS and MF.

The trial paper was submitted for publication, but not published at the time of the review

\section{Risk of bias}

\begin{tabular}{lll}
\hline Bias & Authors' judgement & Support for judgement \\
\hline $\begin{array}{l}\text { Random sequence genera- } \\
\text { tion (selection bias) }\end{array}$ & Low risk & Computer-generated randomisation with variable block size \\
\hline $\begin{array}{l}\text { Allocation concealment } \\
\text { (selection bias) }\end{array}$ & Low risk & Concealed through computer-generated list \\
\hline $\begin{array}{l}\text { Baseline outcome mea- } \\
\text { surement }\end{array}$ & Low risk & Baseline IADL score reported \\
\hline $\begin{array}{l}\text { Baseline characteristics } \\
\text { Blinding of outcome as- } \\
\text { sessment (detection bias) }\end{array}$ & Low risk & Described and evenly distributed \\
\hline
\end{tabular}


Strøm 2017a (Continued)

All outcomes i.e. mortality and obtained by up-to-date national register, but secondary outcomes were obtained by research personnel that were not blinded to treatment allocation

$\begin{aligned} & \text { Incomplete outcome data } \\ & \text { (attrition bias) }\end{aligned}$
All outcomes

\begin{tabular}{lll}
\hline Contamination & High risk & $\begin{array}{l}\text { A significant proportion of participants in the SSU (23\%) were transferred to } \\
\text { the internal medicine department during their hospital stay }\end{array}$ \\
\hline $\begin{array}{l}\text { Selective reporting (re- } \\
\text { porting bias) }\end{array}$ & Low risk & $\begin{array}{l}\text { Protocol published, all outcome data measured and reported as stated in pro- } \\
\text { tocol }\end{array}$ \\
\hline Other bias & Low risk & $\begin{array}{l}\text { The trial appeared to be free of other components that could put it at risk of } \\
\text { bias }\end{array}$ \\
\hline $\begin{array}{l}\text { Overall risk of bias } \\
\text { All outcomes }\end{array}$ & High risk & $\begin{array}{l}\text { High risk of bias and/or unclear risk of bias in one or more domains: blinding of } \\
\text { outcome assessment, incomplete outcome data, or selective reporting }\end{array}$ \\
\hline
\end{tabular}

Sun 2014

Parallel randomised trial
Aim of trial: to test the primary hypotheses that an ED observation protocol would reduce hospital ad-
missions and hospital length-of-stay
Setting: multicentre trial conducted in university-affiliated both public and private EDs (5 sites: Los An-
geles (Public), Boston, Royal Oaks, Troy, Durham, USA), March 2010-October 2011
Number of nurses/bed: unclear
Number of physicians/bed: unclear
Type of SSU: multipurpose units

Participants

Participants with syncope or near-syncope and intermediate risk for subsequent serious outcomes

Inclusion criteria: age $\geq 50$ years, classified to have intermediate risk for subsequent serious outcomes (risk stratification by semi-structured criteria based upon specialty society criteria)

Exclusion criteria: serious condition identified during the ED (e.g. symptomatic arrhythmias, MI, pulmonary embolism), seizure, head trauma, or intoxication as the reason for loss of consciousness, new or baseline cognitive impairment, do-not-resuscitate or do-not-intubate status, active chemotherapy for cancer, and inability to speak either English or Spanish

Numbers recruited: 124, 62 in intervention group vs 62 in usual-care group

Participants analysed for the primary outcome: 124

Mean age: 65 (SD 11) in intervention group vs 64 (SD 11) in usual-care group

Male: 29 (47)\% in intervention group vs 32 (52)\% in usual-care group

Interventions

Intervention: standardised protocol in ED observation unit, consisting of minimum $12 \mathrm{~h}$ of continuous cardiac monitoring, serial troponin test (minimum 2 times), and resting echocardiogram if participants had a cardiac murmur or if a prior rest echocardiogram had not been performed in the prior 6 months. The ED treating teams could perform additional testing at their discretion. The maximum stay in the ED observation unit could not exceed $24 \mathrm{~h}$. Observation protocol participants who were diagnosed with a serious condition, had persistent symptoms of syncope or near-syncope, were felt by the treating 
Sun 2014 (Continued)

physician to be unable to be safely discharged home because of functional reasons (e.g. inability to ambulate), or had pending

tests at $24 \mathrm{~h}$ were admitted to the hospital. All other participants were eligible for discharge. The treat-

ing ED team made the final decision to admit or discharge participants.

Usual care: routine inpatient admission, inpatient medicine service managed participants. The trial protocol did not guide the care of participants

\begin{tabular}{ll}
\hline Outcomes & Primary outcome: hospitalisation rate and hospital length-of-stay (hours) \\
& Secondary outcomes: hospital costs, QoL, serious clinical events, patient satisfaction \\
\hline Notes & Defined time limit of maximum stay in the SSU: $24 \mathrm{~h}$ \\
& Follow-up: 6 months \\
& Trial registration: NCT01003262 \\
& Received additional information from trial authors by 28 February 2017 \\
& $\begin{array}{l}\text { For serious clinical events, additional information on participants lost to follow-up was presented in } \\
\text { supplementary online e-material. We re-calculated serious outcomes by adding 'in-hospital serious } \\
\text { clinical events' to 'after hospital discharge serious clinical events' }\end{array}$
\end{tabular}

\section{Risk of bias}

\begin{tabular}{|c|c|c|}
\hline Bias & Authors' judgement & Support for judgement \\
\hline $\begin{array}{l}\text { Random sequence genera- } \\
\text { tion (selection bias) }\end{array}$ & Low risk & Computer-generated block randomisation sequence by site \\
\hline $\begin{array}{l}\text { Allocation concealment } \\
\text { (selection bias) }\end{array}$ & Low risk & Allocation concealment by a computer-generated list \\
\hline $\begin{array}{l}\text { Baseline outcome mea- } \\
\text { surement }\end{array}$ & Unclear risk & No baseline measure of outcome QoL reported \\
\hline Baseline characteristics & Low risk & Described and evenly distributed \\
\hline $\begin{array}{l}\text { Blinding of outcome as- } \\
\text { sessment (detection bias) } \\
\text { All outcomes }\end{array}$ & High risk & Outcome assessors were not blinded \\
\hline
\end{tabular}

\begin{tabular}{|c|c|c|}
\hline $\begin{array}{l}\text { Incomplete outcome data } \\
\text { (attrition bias) } \\
\text { All outcomes }\end{array}$ & Unclear risk & $\begin{array}{l}\text { Authors explicitly reported loss-to-follow-up data in a supplementary appen- } \\
\text { dix, for primary outcomes hospital admission no participants were missing; } \\
\text { for the other primary outcomes, length of stay in hospital, } 2 \text { participants in } \\
\text { usual-care group were missing ( } 2 \%) \text {. For } 30 \text {-day serious outcomes after hospi- } \\
\text { tal discharge, } 3(5 \%) \text { in intervention group vs } 5(5 \%) \text { in usual-care group were } \\
\text { missing, and for QoL (quality of well-being score), } 14(23 \%) \text { in intervention } \\
\text { group vs } 21 \text { (34\%) in usual-care group were missing, hence more than } 15 \% \\
\text { missing data (as defined in our 'Risk of bias' assessment). Missing data seemed } \\
\text { equally distributed. }\end{array}$ \\
\hline
\end{tabular}

\begin{tabular}{lll}
\hline Contamination & High risk & Participants were transferred to inpatient care from SSU if needed \\
\hline $\begin{array}{l}\text { Selective reporting (re- } \\
\text { porting bias) }\end{array}$ & Low risk & $\begin{array}{l}\text { All outcomes reported in trial registry and methods section were reported } \\
\text { with exception of 6-month QoL data and the formal cost-effectiveness analy- } \\
\text { sis (i.e., comparison of the ratio of cost to quality-adjusted life-year), these } \\
\text { were secondary outcomes and the reasons for not assessing outcomes were } \\
\text { clearly stated (for QoL: this was dropped because of participant complaints }\end{array}$ \\
\end{tabular}


Sun 2014 (Continued)

about survey length and burden; for formal cost-effectiveness analysis: this was dropped because the analysis would not yield additional information compared with the cost-difference analysis)

\begin{tabular}{lll}
\hline Other bias & Unclear risk & $\begin{array}{l}\text { Potential risk of academic bias (Baugh) } \\
\text { Trial authors lowered age inclusion criteria from } 60 \text { years to } 50 \text { years due to } \\
\text { low recruitment rate during the trial period }\end{array}$ \\
\hline $\begin{array}{l}\text { Overall risk of bias } \\
\text { All outcomes }\end{array}$ & High risk & $\begin{array}{l}\text { High risk of bias and/or unclear risk of bias in } 1 \text { or more domains: blinding of } \\
\text { outcome assessment, incomplete outcome data, or selective reporting }\end{array}$
\end{tabular}

\section{Than 2014}

Parallel randomised trial
Aim of trial: to compare the effectiveness of a rapid diagnostic pathway with a standard-care diagnostic
pathway for the assessment of participants with possible cardiac chest pain in a usual clinical practice
setting
Setting: single-centre trial conducted in an urban, tertiary, public, university-affiliated hospital,
Christchurch, New Zealand, October 2010-July 2012
Number of nurses/bed: unclear
Number of physicians/bed: unclear
Type of SSU: multipurpose unit

\section{Participants}

Participants with chest pain consistent with ACS

Inclusion criteria: $\geq 18$ years, symptoms consistent with ACS and physician planned further observation/troponin testing for possible MI

Exclusion criteria: sT-elevation MI, clear other cause than ACS, inability to provide consent, staff considered recruitment to be inappropriate, chest pain symptoms $>12 \mathrm{~h}$, transfer from other hospital, pregnancy, previous inclusion in trial or inability to be discharged after hospitalisation

Numbers recruited: 544, 271 to the intervention group vs 273 to the usual-care group

Participants analysed for the primary outcome: 542

Mean age: 60.5 (SD 12.6) in intervention group vs 60.5 (SD 12.6) in usual-care group

Male: $171(63) \%$ in intervention group vs $166(61) \%$ in usual-care group (TIMI), ECG and troponin testing; if TIMI was 0 participants were placed in observation unit for $2 \mathrm{~h}$ and ECG and troponin tests were repeated, if these tests were negative the participant was discharged and scheduled to a $72 \mathrm{~h}$ outpatient treadmill test. If participant had TIMI score $>0$ or positive test results at any time, participants were admitted)

Usual care: standard pathway by cardiology service (initial troponin and ECG, prolonged observation and a second troponin test 6-12 h after onset of pain, observation in inward unit. Follow-up appointments depended on the clinician, usually 7 -day follow-up with general practitioner)

Outcomes 
Than 2014 (Continued)

Secondary outcomes: defined in trial registration to be length of stay in hospital, serious adverse events, proportion of participants that ultimately diagnosed as having acute MI, cost-effectiveness, health utility, satisfaction

Notes

Defined time limit of maximum stay in the SSU: 12-16 h, occasionally longer (information provided by trial authors)

Follow-up: 30 days

Trial registration: ACTRN12610000766011

Received additional information from trial authors by 28 February 2017 and 13 July 2017

\section{Risk of bias}

\begin{tabular}{|c|c|c|}
\hline Bias & Authors' judgement & Support for judgement \\
\hline $\begin{array}{l}\text { Random sequence genera- } \\
\text { tion (selection bias) }\end{array}$ & Low risk & Computer-generated block randomisation sequence \\
\hline $\begin{array}{l}\text { Allocation concealment } \\
\text { (selection bias) }\end{array}$ & Low risk & Sealed envelopes \\
\hline $\begin{array}{l}\text { Baseline outcome mea- } \\
\text { surement }\end{array}$ & Low risk & Baseline outcome measurement not relevant \\
\hline Baseline characteristics & Low risk & Described and evenly distributed \\
\hline $\begin{array}{l}\text { Blinding of outcome as- } \\
\text { sessment (detection bias) } \\
\text { All outcomes }\end{array}$ & Low risk & Assessors were blinded for treatment allocations \\
\hline $\begin{array}{l}\text { Incomplete outcome data } \\
\text { (attrition bias) } \\
\text { All outcomes }\end{array}$ & Low risk & $\begin{array}{l}2 \text { participants were recruited twice ( } 1 \text { in each group) and excluded, these were } \\
\text { clearly described and }<15 \% \text { of included participants, outcomes for all other } \\
\text { participants were reported }\end{array}$ \\
\hline Contamination & High risk & $\begin{array}{l}41 \text { participants in intervention group received usual care, and } 3 \text { participants in } \\
\text { usual-care group received intervention care }\end{array}$ \\
\hline $\begin{array}{l}\text { Selective reporting (re- } \\
\text { porting bias) }\end{array}$ & Unclear risk & $\begin{array}{l}\text { In the trial registration, trial authors reported to assess additional outcomes } \\
\text { that were not reported in the manuscript (EQ-5D, costs, satisfaction) for rea- } \\
\text { sons not stated, we received the results of these outcomes after contact with } \\
\text { trial authors }\end{array}$ \\
\hline \multirow[t]{2}{*}{ Other bias } & Unclear risk & $\begin{array}{l}\text { Potential risk of academic bias (trial author Goodacre had published several } \\
\text { trials on SSUs) }\end{array}$ \\
\hline & & $\begin{array}{l}\text { The trial appeared to be free of other components that could put it at risk of } \\
\text { bias. }\end{array}$ \\
\hline $\begin{array}{l}\text { Overall risk of bias } \\
\text { All outcomes }\end{array}$ & High risk & $\begin{array}{l}\text { High risk of bias and/or unclear risk of bias in } 1 \text { or more domains: blinding of } \\
\text { outcome assessment, incomplete outcome data, or selective reporting }\end{array}$ \\
\hline
\end{tabular}

ACC: American College of Cardiology; ACS: acute coronary syndrome; AF: atrial fibrillation; AHA: American Heart Association; BP: blood pressure; bpm: beats per minute; CBC: complete blood count CCI: Charlson comorbidity index CPU: chest pain unit; CKMB: Creatine Kinase Myocardial B-fraction; CT: computed tomography; IADL: instrumental activities of daily living; iv: intravenous; ECG: electrocardiogram; ED: emergency department; IQR: interquartile range; MI: myocardial infarction; mph: miles per hour; MRI: magnetic resonance imaging; NA: not applicable; NYHA: New York Heart Association; OARS-IADL: Older Americans Resources and Services instrumental Activities of Daily Living-score; QoL: quality of life; SD: standard deviation; SSU: short-stay unit 
Characteristics of excluded studies [ordered by study ID]

\begin{tabular}{|c|c|}
\hline Study & Reason for exclusion \\
\hline Abdelaziz 2016 & Ineligible intervention \\
\hline Aggarwal 1995 & Ineligible study design \\
\hline Aitken 1993 & Ineligible intervention \\
\hline Alonso 2010 & Ineligible study design \\
\hline Applegate 1990 & Ineligible intervention \\
\hline Applegate 1991 & Ineligible intervention \\
\hline Arendts 2006 & Ineligible study design \\
\hline Asplund 2000 & Ineligible intervention \\
\hline Barbado 1999 & Ineligible study design \\
\hline Barberger-Gateau 1986 & Ineligible study design \\
\hline Barberger-Gateau 1989 & Ineligible study design \\
\hline Barnes 2012 & Ineligible intervention \\
\hline Basic 2009 & Ineligible study design \\
\hline Baugh 2011 & Ineligible study design \\
\hline Baugh 2012 & Ineligible study design \\
\hline Baugh 2014 & Ineligible study design \\
\hline Blecker 2014 & Ineligible study design \\
\hline Bogaty 2001 & Ineligible comparator \\
\hline Broquetas 2008 & Ineligible study design \\
\hline Bullard 2007 & $\begin{array}{l}\text { Ineligible study design; quasi-randomised trial, but randomised days not participants to } \\
\text { either SSU care or traditional care }\end{array}$ \\
\hline Burkhardt 2005 & Ineligible study design \\
\hline Carpentier 2001 & Ineligible study design \\
\hline Caterino 2013 & Ineligible study design \\
\hline Chen 2005 & Ineligible study design \\
\hline Cheng 2016 & Ineligible study design \\
\hline Choi 1999 & Ineligible study design \\
\hline
\end{tabular}




\begin{tabular}{|c|c|}
\hline Study & Reason for exclusion \\
\hline Claesson 2000 & Ineligible intervention \\
\hline Claesson 2003 & Ineligible study design \\
\hline Clemson 2016 & Ineligible intervention \\
\hline Cochard 1999 & Ineligible study design \\
\hline Collier 2007 & Ineligible intervention \\
\hline Collins 2013 & Ineligible study design \\
\hline Corbella 2002 & Ineligible study design \\
\hline Covinsky 1997 & Ineligible intervention \\
\hline Cross 2010 & Ineligible study design \\
\hline Cruz 2001 & Ineligible study design \\
\hline Dallos 1981 & Ineligible study design \\
\hline Deng 2004 & Ineligible intervention \\
\hline Diagana 2008 & Ineligible study design \\
\hline Downing 2008 & Ineligible study design \\
\hline Ekdahl 2014 & Ineligible intervention \\
\hline Ekerstad 2017 & Ineligible intervention \\
\hline Fagerberg 2000 & Ineligible intervention \\
\hline Farkouh 1997 & Copy of another reference found in search \\
\hline Fayas 2013 & Ineligible study design \\
\hline Fung 2007 & Ineligible study design \\
\hline Furlanetto 2014 & Ineligible study design \\
\hline Gaspoz 1994 & Ineligible study design \\
\hline Germain 1995 & Ineligible intervention \\
\hline Ghaemmaghami 2009 & Ineligible study design \\
\hline Goodacre 1998 & Ineligible study design \\
\hline Goodacre 2004 & Ineligible study design (quasi-randomised trial) \\
\hline Goodacre 2007 & Ineligible study design \\
\hline Harper 1988 & Ineligible study design \\
\hline
\end{tabular}




\begin{tabular}{|c|c|}
\hline Study & Reason for exclusion \\
\hline Harris 1991 & Ineligible intervention \\
\hline Harrison 2003 & Ineligible patient population \\
\hline ISRCTN21800480 & Ineligible intervention \\
\hline Jagminas 2005 & Ineligible study design \\
\hline Juan 2006 & Ineligible study design \\
\hline Juan 2010 & Ineligible study design \\
\hline Kam 2008 & Ineligible study design \\
\hline Kelen 2001 & Ineligible study design \\
\hline Khan 1997 & Ineligible study design \\
\hline Koton 2005 & Ineligible intervention \\
\hline Llopis 2015 & Ineligible study design \\
\hline Llopis 2016 & Ineligible study design \\
\hline Mahler 2013 & Ineligible study design \\
\hline Mahler 2015 & Ineligible intervention \\
\hline Martín-Sánchez 2014 & Ineligible study design \\
\hline Michael 2014 & Ineligible study design \\
\hline Miller 2011 & Ineligible comparator \\
\hline Miller 2012 & Copy of another reference found in search \\
\hline Mitchell 2009 & Ineligible study design \\
\hline Muñoz 2006 & Ineligible study design \\
\hline Navarrete 2016 & Ineligible intervention \\
\hline NCT02421133 & Ineligible intervention \\
\hline Ross 2004 & Commentary \\
\hline Rubenstein 1984 & Ineligible intervention \\
\hline Rubenstein 1988 & Ineligible intervention \\
\hline Salazar 2006 & Ineligible study design \\
\hline
\end{tabular}


Characteristics of ongoing studies [ordered by study ID]

\section{NCT03302910}

Trial name or title

Methods
Short Stay Unit vs Hospitalization in Acute Heart Failure (SSU-AHF)

Parallel randomised trial

Aim of trial: to test whether short stay-unit AHF management for $<24 \mathrm{~h}$ increases days-alive-andout-of-hospital, QoL assessment, caregiver burden, and costs compared to inpatient management

Setting: multicentre study ( 3 centres), USA

Number of nurses/bed: unclear

Number of physicians/bed: unclear

Type of SSU: multipurpose unit

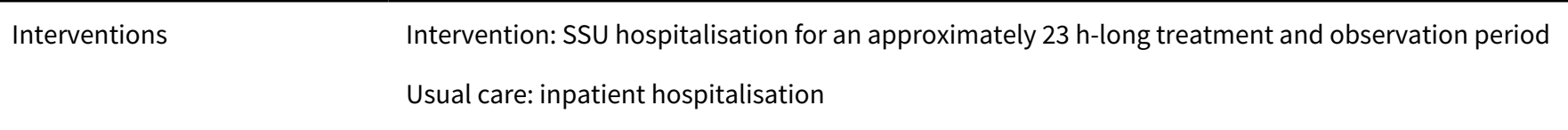

Outcomes Primary outcome: days alive and out of hospital

Secondary outcomes: QoL, cost-effectiveness analysis, caregiver burden, modified resource utilisation questionnaire, all-cause mortality and re-hospitalisation, days alive and out of hospital

\begin{tabular}{ll}
\hline Starting date & December 6th 2017 \\
\hline Contact information & Dr. Peter S. Pang, MD. Phone: $312-515-4025$, email: ppang@iu.edu \\
\hline Notes & Estimated completion date: 31 March 2021 \\
& Trial registration: NCT03302910 \\
\hline
\end{tabular}

ACS: acute coronary syndrome; AHF: acute heart failure; BP: blood pressure; bpm: beats per minute; ED: emergency department; ICU: intensive care unit; $\mathbf{h}$ : hour, iv: intravenous; QoL: quality of life; SSU: short-stay unit

\section{DATA AND ANALYSES}


Comparison 1. Mortality in participants treated in short-stay unit vs usual care

\begin{tabular}{|c|c|c|c|c|}
\hline Outcome or subgroup title & No. of studies & $\begin{array}{l}\text { No. of partici- } \\
\text { pants }\end{array}$ & Statistical method & Effect size \\
\hline $\begin{array}{l}1 \text { Mortality at time point closest to } 90 \\
\text { days }\end{array}$ & 5 & 1294 & $\begin{array}{l}\text { Risk Ratio (M-H, Random, } \\
95 \% \mathrm{Cl})\end{array}$ & $0.73[0.47,1.15]$ \\
\hline $\begin{array}{l}2 \text { Mortality at time point closest to } 90 \\
\text { days best-worst case scenario }\end{array}$ & 5 & 1318 & $\begin{array}{l}\text { Risk Ratio (M-H, Random, } \\
\text { 95\% Cl) }\end{array}$ & $0.57[0.37,0.87]$ \\
\hline $\begin{array}{l}3 \text { Mortality at time point closest to } 90 \\
\text { days worst-best case scenario }\end{array}$ & 5 & 1318 & $\begin{array}{l}\text { Risk Ratio (M-H, Random, } \\
95 \% \mathrm{Cl})\end{array}$ & $1.05[0.57,1.94]$ \\
\hline $\begin{array}{l}4 \text { Mortality at time point closest to } 90 \\
\text { days published trials }\end{array}$ & 3 & 749 & $\begin{array}{l}\text { Risk Ratio (M-H, Random, } \\
95 \% \mathrm{Cl})\end{array}$ & $0.52[0.13,2.08]$ \\
\hline $\begin{array}{l}5 \text { Mortality at time point closest to } 90 \\
\text { days and outcome assessed within } 6 \\
\text { months of randomisation }\end{array}$ & 4 & 1191 & $\begin{array}{l}\text { Risk Ratio (M-H, Random, } \\
95 \% \mathrm{Cl})\end{array}$ & $0.74[0.46,1.18]$ \\
\hline $\begin{array}{l}6 \text { Mortality at time point closest to } 90 \\
\text { days multipurpose unit vs specialised } \\
\text { unit }\end{array}$ & 5 & 1294 & $\begin{array}{l}\text { Risk Ratio (M-H, Random, } \\
95 \% \mathrm{Cl})\end{array}$ & $0.73[0.47,1.15]$ \\
\hline 6.1 Multipurpose unit & 3 & 767 & $\begin{array}{l}\text { Risk Ratio (M-H, Random, } \\
95 \% \mathrm{Cl})\end{array}$ & $0.75[0.47,1.21]$ \\
\hline 6.2 Specialised unit & 2 & 527 & $\begin{array}{l}\text { Risk Ratio (M-H, Random, } \\
95 \% \mathrm{Cl} \text { ) }\end{array}$ & $0.58[0.12,2.67]$ \\
\hline $\begin{array}{l}7 \text { Mortality at time point closest to } 90 \\
\text { days non-protocol-based vs proto- } \\
\text { col-based care }\end{array}$ & 5 & 1294 & $\begin{array}{l}\text { Risk Ratio (M-H, Random, } \\
95 \% \mathrm{Cl})\end{array}$ & $0.73[0.47,1.15]$ \\
\hline 7.1 Non-protocol-based care & 2 & 545 & $\begin{array}{l}\text { Risk Ratio (M-H, Random, } \\
95 \% \mathrm{Cl})\end{array}$ & $0.81[0.43,1.52]$ \\
\hline 7.2 Protocol-based care & 3 & 749 & $\begin{array}{l}\text { Risk Ratio (M-H, Random, } \\
95 \% \mathrm{Cl})\end{array}$ & $0.52[0.13,2.08]$ \\
\hline $\begin{array}{l}8 \text { Mortality at time point closest to } 90 \\
\text { days older participants vs younger par- } \\
\text { ticipants }\end{array}$ & 5 & 1294 & $\begin{array}{l}\text { Risk Ratio (M-H, Random, } \\
95 \% \mathrm{Cl})\end{array}$ & $0.73[0.47,1.15]$ \\
\hline 8.1 Older participants & 2 & 545 & $\begin{array}{l}\text { Risk Ratio (M-H, Random, } \\
95 \% \mathrm{Cl})\end{array}$ & $0.81[0.43,1.52]$ \\
\hline 8.2 Younger participants & 3 & 749 & $\begin{array}{l}\text { Risk Ratio (M-H, Random, } \\
95 \% \mathrm{Cl})\end{array}$ & $0.52[0.13,2.08]$ \\
\hline 9 Mortality at maximum follow-up & 5 & 1277 & $\begin{array}{l}\text { Risk Ratio (M-H, Fixed, 95\% } \\
\mathrm{Cl})\end{array}$ & $0.84[0.62,1.13]$ \\
\hline $\begin{array}{l}10 \text { Mortality at maximum follow-up } \\
\text { best-worst case scenario }\end{array}$ & 5 & 1318 & $\begin{array}{l}\text { Risk Ratio (M-H, Random, } \\
95 \% \mathrm{Cl})\end{array}$ & $0.67[0.50,0.88]$ \\
\hline $\begin{array}{l}11 \text { Mortality at maximum follow-up } \\
\text { worst-best case scenario }\end{array}$ & 5 & 1318 & $\begin{array}{l}\text { Risk Ratio (M-H, Random, } \\
95 \% \mathrm{Cl})\end{array}$ & $1.17[0.73,1.89]$ \\
\hline
\end{tabular}




\begin{tabular}{|c|c|c|c|c|}
\hline Outcome or subgroup title & No. of studies & $\begin{array}{l}\text { No. of partici- } \\
\text { pants }\end{array}$ & Statistical method & Effect size \\
\hline $\begin{array}{l}12 \text { Mortality at maximum follow-up } \\
\text { published trials }\end{array}$ & 3 & 732 & $\begin{array}{l}\text { Risk Ratio (M-H, Random, } \\
95 \% \mathrm{Cl} \text { ) }\end{array}$ & $0.92[0.51,1.66]$ \\
\hline $\begin{array}{l}13 \text { Mortality at maximum follow-up } \\
\text { and outcome assessed within } 6 \\
\text { months of randomisation }\end{array}$ & 4 & 1174 & $\begin{array}{l}\text { Risk Ratio (M-H, Random, } \\
95 \% \mathrm{Cl})\end{array}$ & $0.84[0.62,1.13]$ \\
\hline $\begin{array}{l}14 \text { Mortality at maximum follow-up } \\
\text { multipurpose unit vs specialised unit }\end{array}$ & 5 & 1277 & $\begin{array}{l}\text { Risk Ratio (M-H, Fixed, 95\% } \\
\mathrm{Cl} \text { ) }\end{array}$ & $0.84[0.62,1.13]$ \\
\hline 14.1 Multipurpose unit & 3 & 767 & $\begin{array}{l}\text { Risk Ratio (M-H, Fixed, 95\% } \\
\mathrm{Cl} \text { ) }\end{array}$ & $0.80[0.57,1.13]$ \\
\hline 14.2 Specialised unit & 2 & 510 & $\begin{array}{l}\text { Risk Ratio (M-H, Fixed, 95\% } \\
\mathrm{Cl})\end{array}$ & $0.95[0.52,1.73]$ \\
\hline $\begin{array}{l}15 \text { Mortality at maximum follow-up } \\
\text { non-protocol-based vs protocol-based } \\
\text { care }\end{array}$ & 5 & 1277 & $\begin{array}{l}\text { Risk Ratio (M-H, Fixed, 95\% } \\
\mathrm{Cl})\end{array}$ & $0.84[0.62,1.13]$ \\
\hline 15.1 Non-protocol-based care & 2 & 545 & $\begin{array}{l}\text { Risk Ratio (M-H, Fixed, 95\% } \\
\mathrm{Cl} \text { ) }\end{array}$ & $0.81[0.58,1.15]$ \\
\hline 15.2 Protocol-based care & 3 & 732 & $\begin{array}{l}\text { Risk Ratio (M-H, Fixed, 95\% } \\
\mathrm{Cl} \text { ) }\end{array}$ & $0.91[0.50,1.63]$ \\
\hline $\begin{array}{l}16 \text { Mortality at maximum follow-up } \\
\text { older vs younger participants }\end{array}$ & 5 & 1277 & $\begin{array}{l}\text { Risk Ratio (M-H, Fixed, 95\% } \\
\mathrm{Cl})\end{array}$ & $0.84[0.62,1.13]$ \\
\hline 16.1 Older participants & 2 & 545 & $\begin{array}{l}\text { Risk Ratio (M-H, Fixed, 95\% } \\
\mathrm{Cl})\end{array}$ & $0.81[0.58,1.15]$ \\
\hline 16.2 Younger participants & 3 & 732 & $\begin{array}{l}\text { Risk Ratio (M-H, Fixed, 95\% } \\
\mathrm{Cl} \text { ) }\end{array}$ & $0.91[0.50,1.63]$ \\
\hline
\end{tabular}

Analysis 1.1. Comparison 1 Mortality in participants treated in shortstay unit vs usual care, Outcome 1 Mortality at time point closest to 90 days.

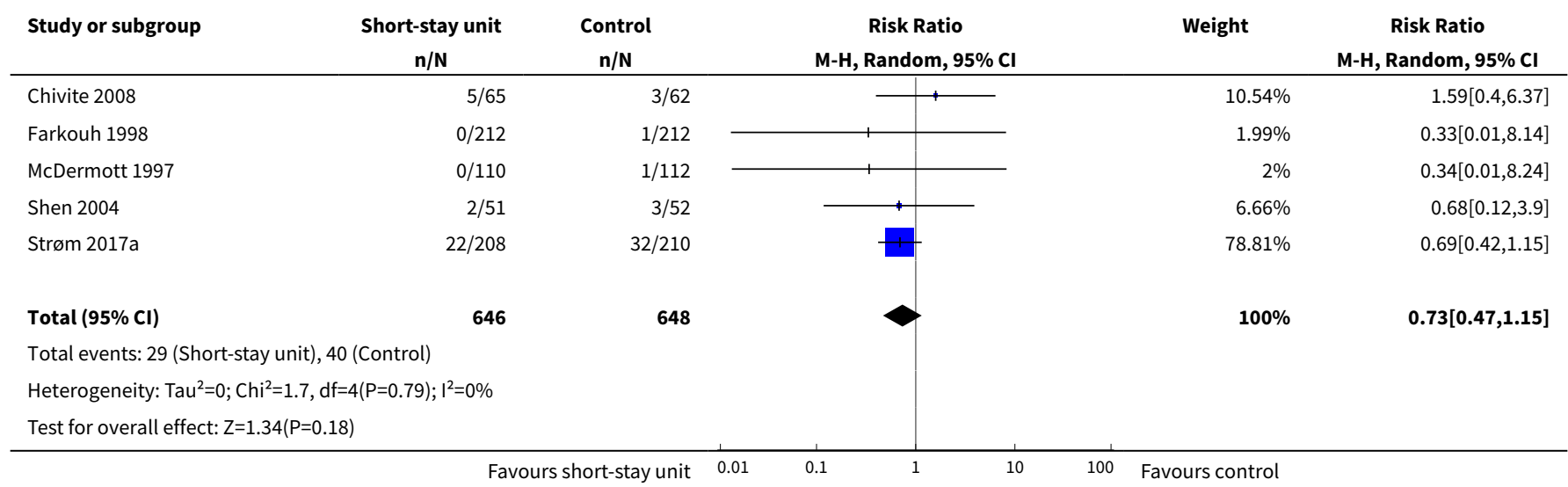


Analysis 1.2. Comparison 1 Mortality in participants treated in short-stay unit vs usual care, Outcome 2 Mortality at time point closest to 90 days best-worst case scenario.

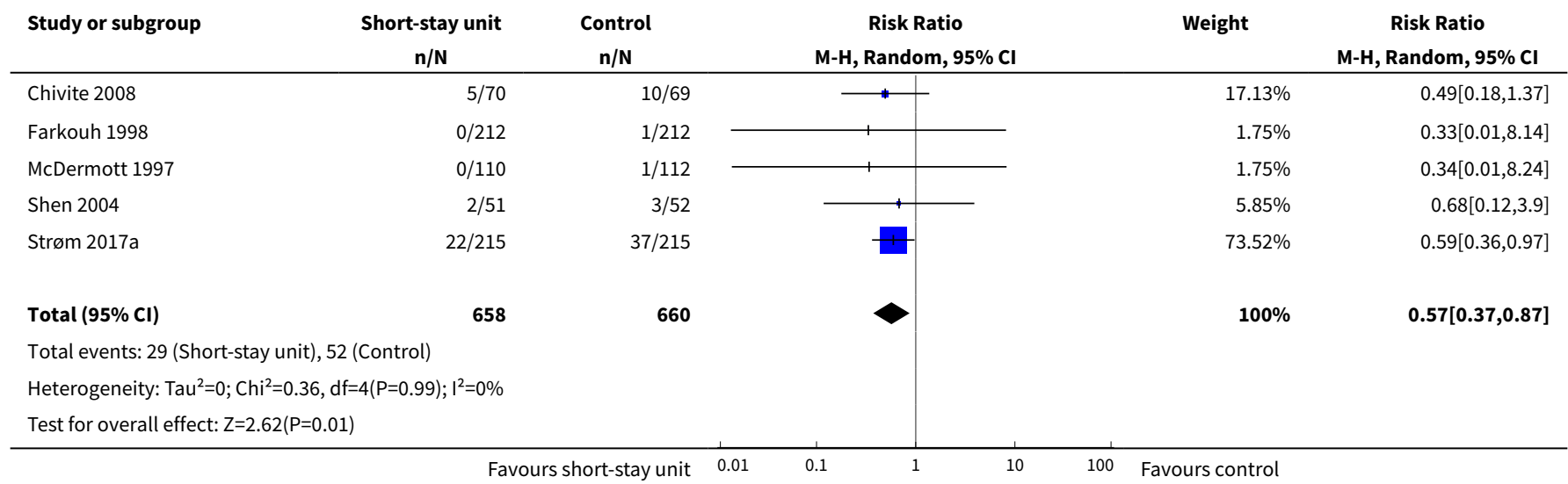

Analysis 1.3. Comparison 1 Mortality in participants treated in short-stay unit vs usual care, Outcome 3 Mortality at time point closest to 90 days worst-best case scenario.

\begin{tabular}{|c|c|c|c|c|c|}
\hline Study or subgroup & $\begin{array}{c}\text { Short-stay unit } \\
\mathrm{n} / \mathrm{N}\end{array}$ & $\begin{array}{c}\text { Control } \\
\mathrm{n} / \mathrm{N}\end{array}$ & $\begin{array}{c}\text { Risk Ratio } \\
\text { M-H, Random, } 95 \% \mathrm{CI}\end{array}$ & Weight & $\begin{array}{c}\text { Risk Ratio } \\
\text { M-H, Random, } 95 \% \text { CI }\end{array}$ \\
\hline Chivite 2008 & $10 / 70$ & $3 / 69$ & $\rightarrow$ & $19.39 \%$ & $3.29[0.94,11.43]$ \\
\hline Farkouh 1998 & $0 / 212$ & $1 / 212$ & & $3.55 \%$ & $0.33[0.01,8.14]$ \\
\hline McDermott 1997 & $0 / 110$ & $1 / 112$ & & $3.56 \%$ & $0.34[0.01,8.24]$ \\
\hline Shen 2004 & $2 / 51$ & $3 / 52$ & & $10.94 \%$ & $0.68[0.12,3.9]$ \\
\hline Strøm 2017a & $29 / 215$ & $32 / 215$ & & $62.56 \%$ & $0.91[0.57,1.44]$ \\
\hline Total $(95 \% \mathrm{Cl})$ & 658 & 660 & & $100 \%$ & $1.05[0.57,1.94]$ \\
\hline \multicolumn{6}{|c|}{ Total events: 41 (Short-stay unit), 40 (Control) } \\
\hline \multicolumn{6}{|c|}{ Heterogeneity: $\operatorname{Tau}^{2}=0.1 ; \mathrm{Chi}^{2}=4.77, \mathrm{df}=4(\mathrm{P}=0.31) ; \mathrm{I}^{2}=16.14 \%$} \\
\hline Test for overall effect & & & & & \\
\hline
\end{tabular}

Analysis 1.4. Comparison 1 Mortality in participants treated in short-stay unit vs usual care, Outcome 4 Mortality at time point closest to 90 days published trials.

\begin{tabular}{|c|c|c|c|c|c|}
\hline Study or subgroup & $\begin{array}{c}\text { Short-stay unit } \\
n / N\end{array}$ & $\begin{array}{c}\text { Control } \\
n / N\end{array}$ & $\begin{array}{c}\text { Risk Ratio } \\
\text { M-H, Random, 95\% Cl }\end{array}$ & Weight & $\begin{array}{c}\text { Risk Ratio } \\
\text { M-H, Random, } 95 \% \mathrm{Cl}\end{array}$ \\
\hline Farkouh 1998 & $0 / 212$ & $1 / 212$ & & $18.7 \%$ & $0.33[0.01,8.14]$ \\
\hline McDermott 1997 & $0 / 110$ & $1 / 112$ & & $18.76 \%$ & $0.34[0.01,8.24]$ \\
\hline Shen 2004 & $2 / 51$ & $3 / 52$ & 4 & $62.54 \%$ & $0.68[0.12,3.9]$ \\
\hline Total $(95 \% \mathrm{Cl})$ & 373 & 376 & & $100 \%$ & $0.52[0.13,2.08]$ \\
\hline \multicolumn{6}{|c|}{ Heterogeneity: $\mathrm{Tau}^{2}=0 ; \mathrm{Chi}^{2}=0.24, \mathrm{df}=2(\mathrm{P}=0.89) ; \mathrm{I}^{2}=0 \%$} \\
\hline Test for overall effect & & & & & \\
\hline
\end{tabular}


Analysis 1.5. Comparison 1 Mortality in participants treated in short-stay unit vs usual care, Outcome 5 Mortality at time point closest to 90 days and outcome assessed within 6 months of randomisation.

\begin{tabular}{|c|c|c|c|c|c|}
\hline Study or subgroup & $\begin{array}{c}\text { Short-stay unit } \\
n / N \\
\end{array}$ & $\begin{array}{c}\text { Control } \\
\mathrm{n} / \mathrm{N}\end{array}$ & $\begin{array}{c}\text { Risk Ratio } \\
\text { M-H, Random, 95\% Cl }\end{array}$ & Weight & $\begin{array}{c}\text { Risk Ratio } \\
\text { M-H, Random, 95\% Cl }\end{array}$ \\
\hline Chivite 2008 & $5 / 65$ & $3 / 62$ & $\rightarrow$ & $11.3 \%$ & $1.59[0.4,6.37]$ \\
\hline Farkouh 1998 & $0 / 212$ & $1 / 212$ & & $2.13 \%$ & $0.33[0.01,8.14]$ \\
\hline McDermott 1997 & $0 / 110$ & $1 / 112$ & & $2.14 \%$ & $0.34[0.01,8.24]$ \\
\hline Strøm 2017a & $22 / 208$ & $32 / 210$ & & $84.43 \%$ & $0.69[0.42,1.15]$ \\
\hline \multicolumn{6}{|c|}{ Total events: 27 (Short-stay unit), 37 (Control) } \\
\hline \multicolumn{6}{|c|}{ Heterogeneity: $\mathrm{Tau}^{2}=0 ; \mathrm{Chi}^{2}=1.7, \mathrm{df}=3(\mathrm{P}=0.64) ; \mathrm{I}^{2}=0 \%$} \\
\hline Test for overall effec & & & & & \\
\hline
\end{tabular}

Analysis 1.6. Comparison 1 Mortality in participants treated in short-stay unit vs usual care, Outcome 6 Mortality at time point closest to 90 days multipurpose unit vs specialised unit.

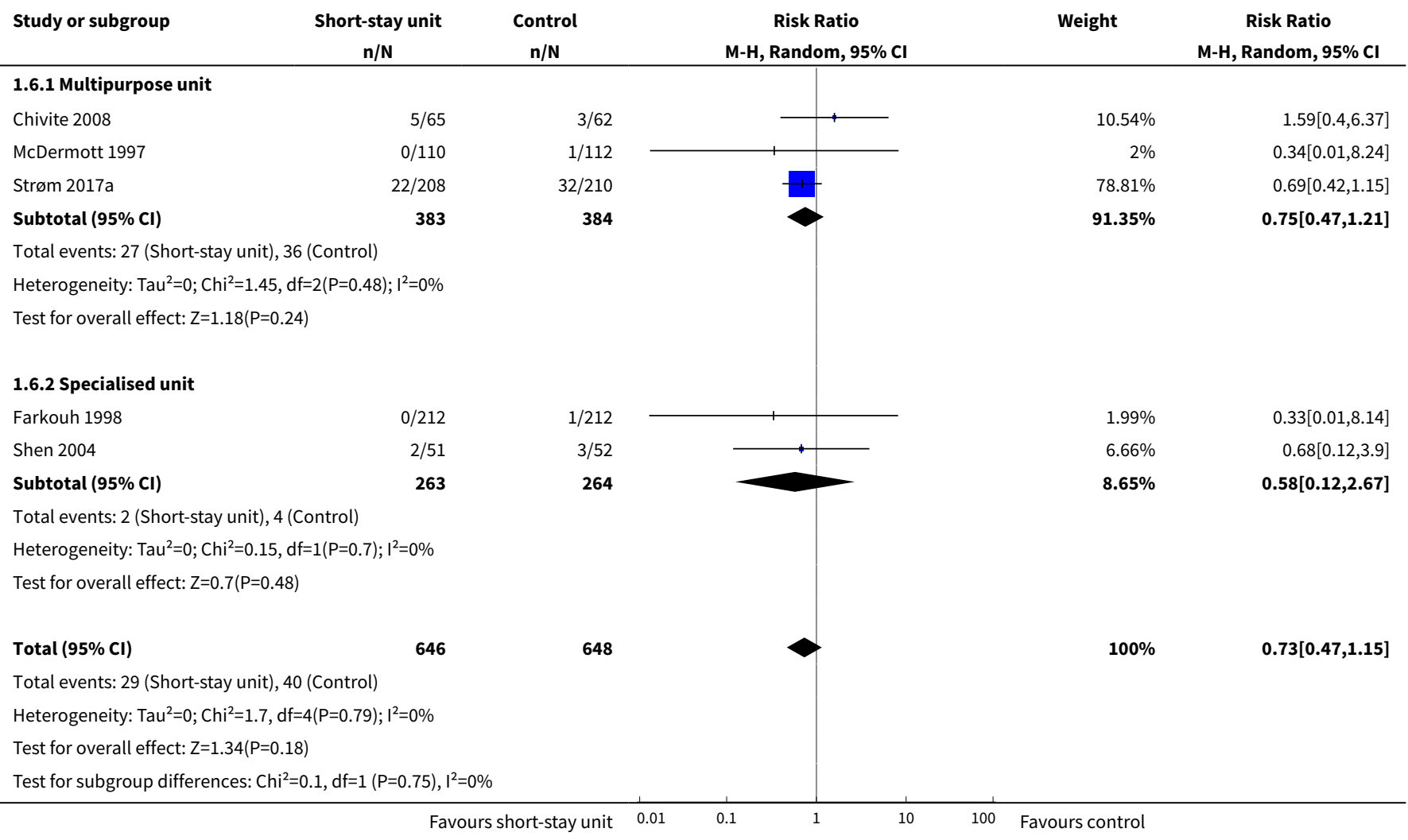


Analysis 1.7. Comparison 1 Mortality in participants treated in short-stay unit vs usual care, Outcome $\mathbf{7}$ Mortality at time point closest to $\mathbf{9 0}$ days non-protocol-based vs protocol-based care.

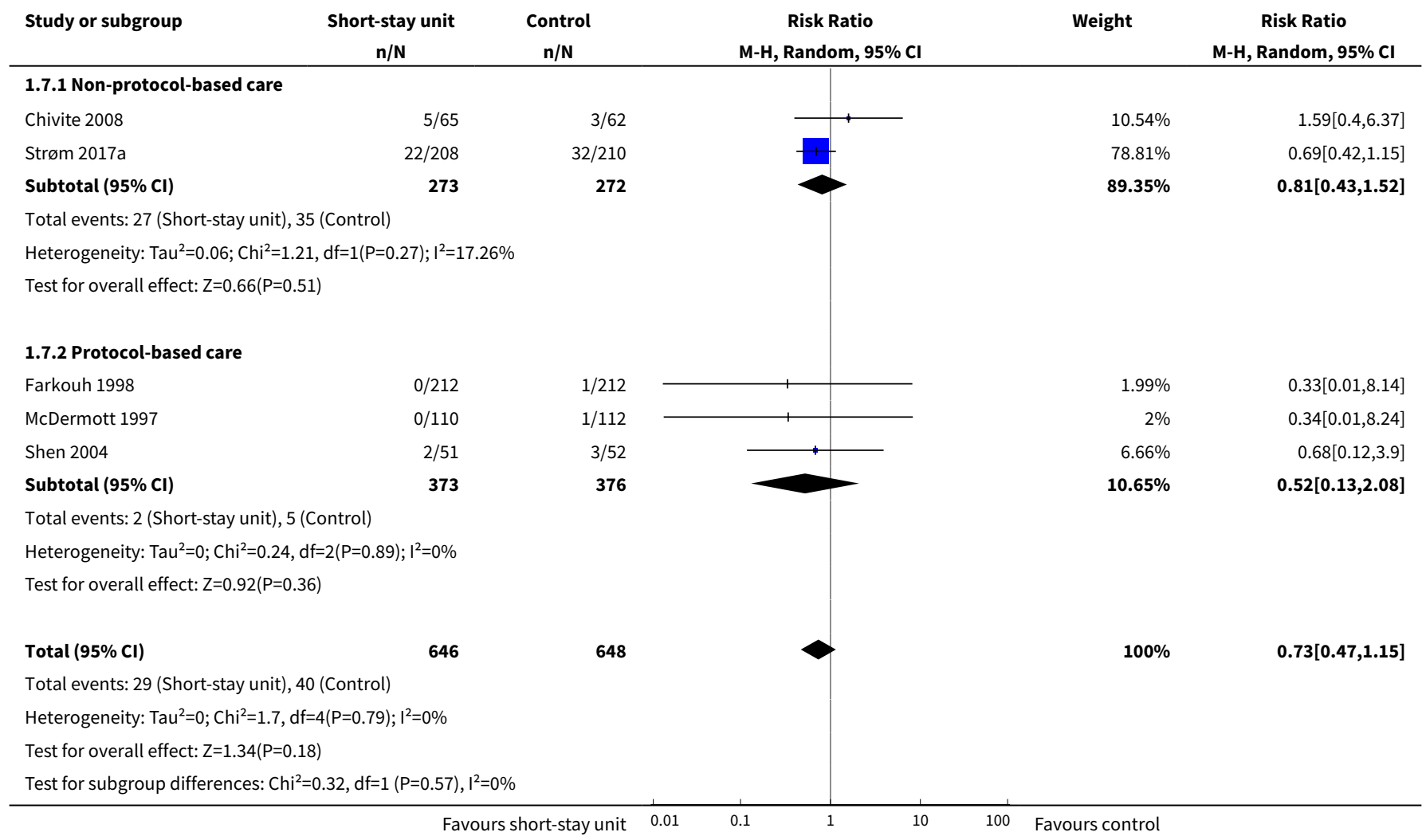

Analysis 1.8. Comparison 1 Mortality in participants treated in short-stay unit vs usual care, Outcome 8 Mortality at time point closest to 90 days older participants vs younger participants.

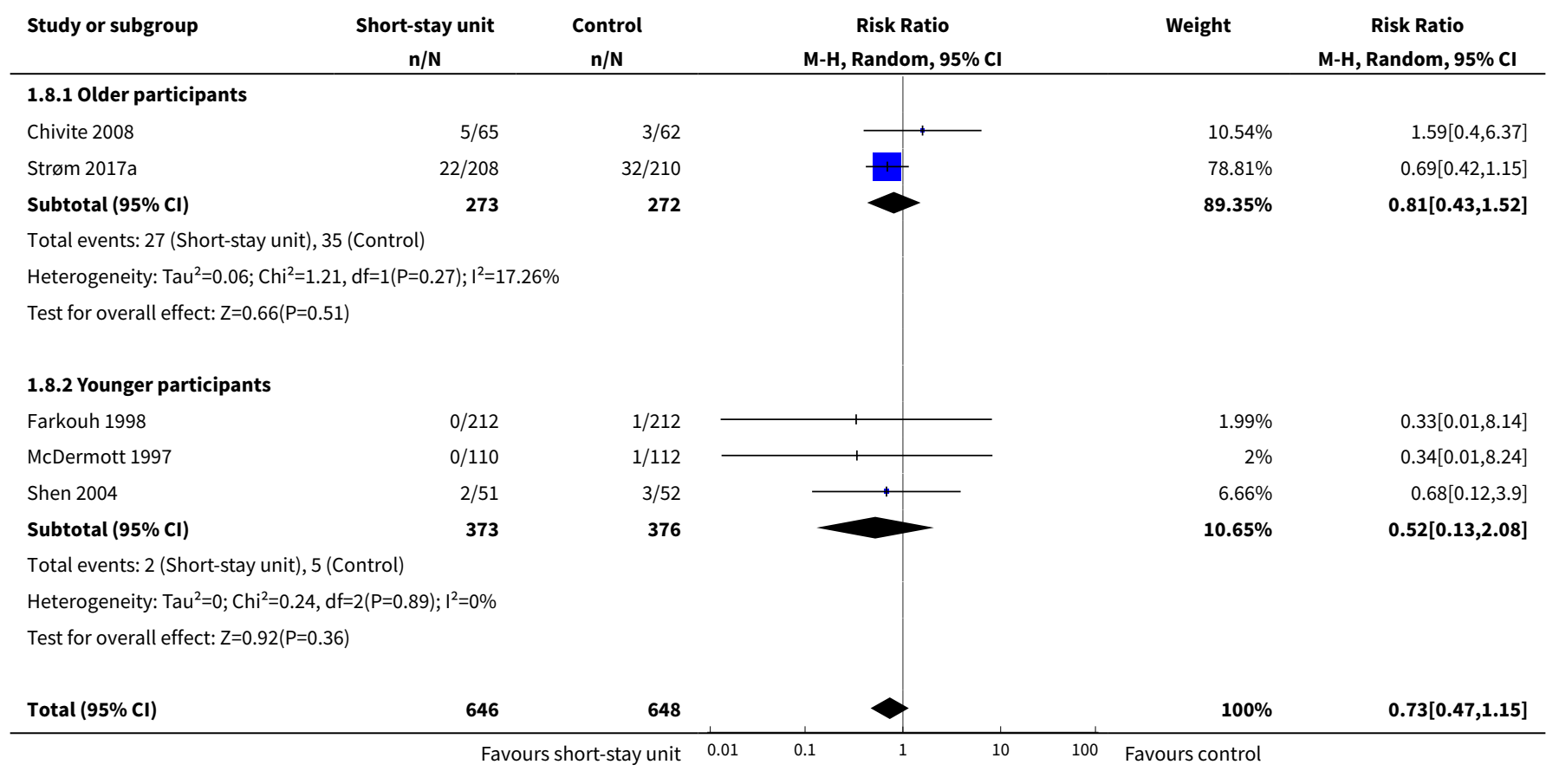




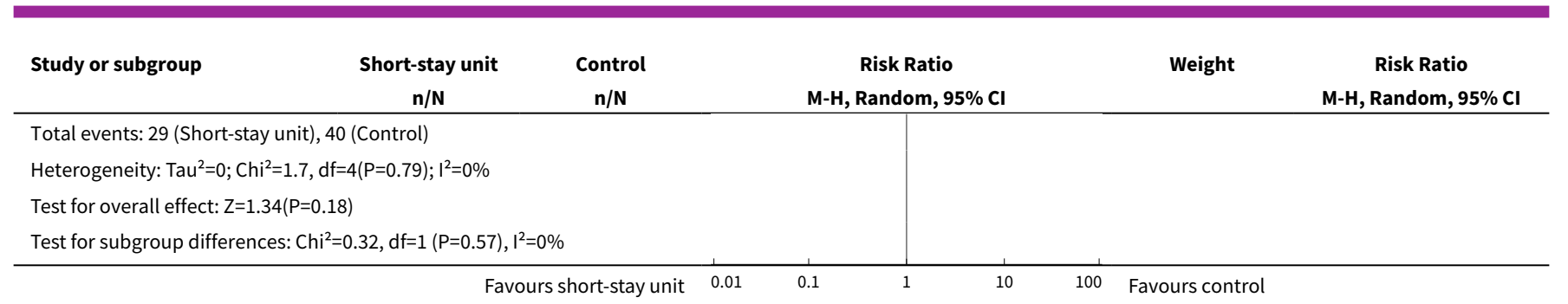

Analysis 1.9. Comparison 1 Mortality in participants treated in shortstay unit vs usual care, Outcome 9 Mortality at maximum follow-up.

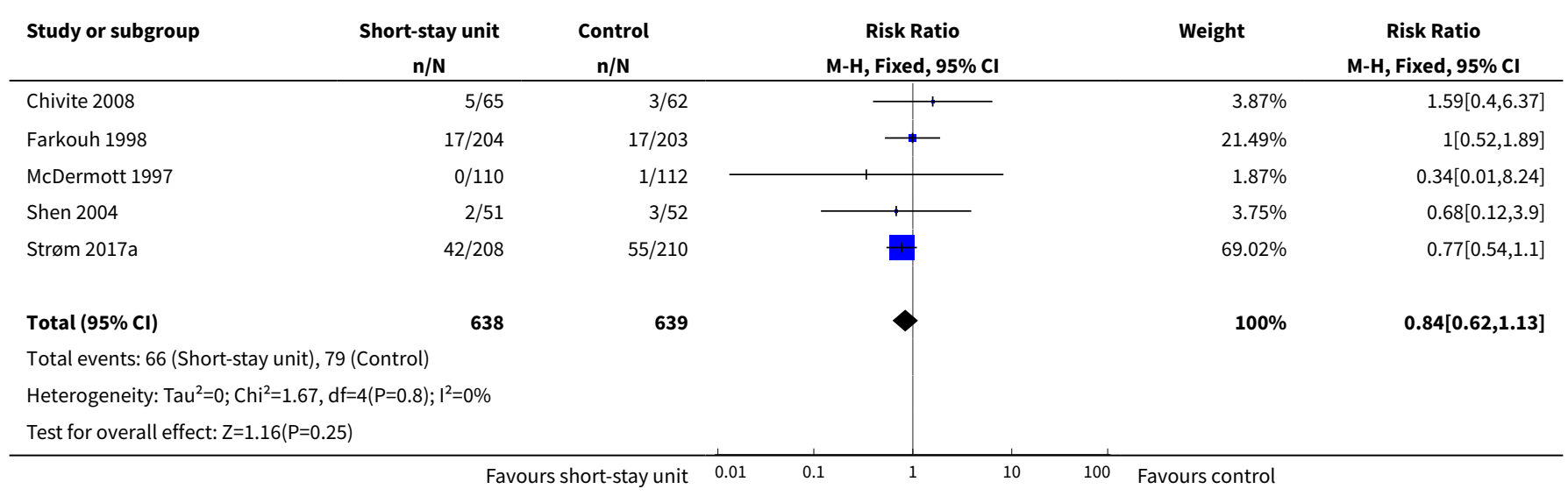

Analysis 1.10. Comparison 1 Mortality in participants treated in short-stay unit vs usual care, Outcome 10 Mortality at maximum follow-up best-worst case scenario.

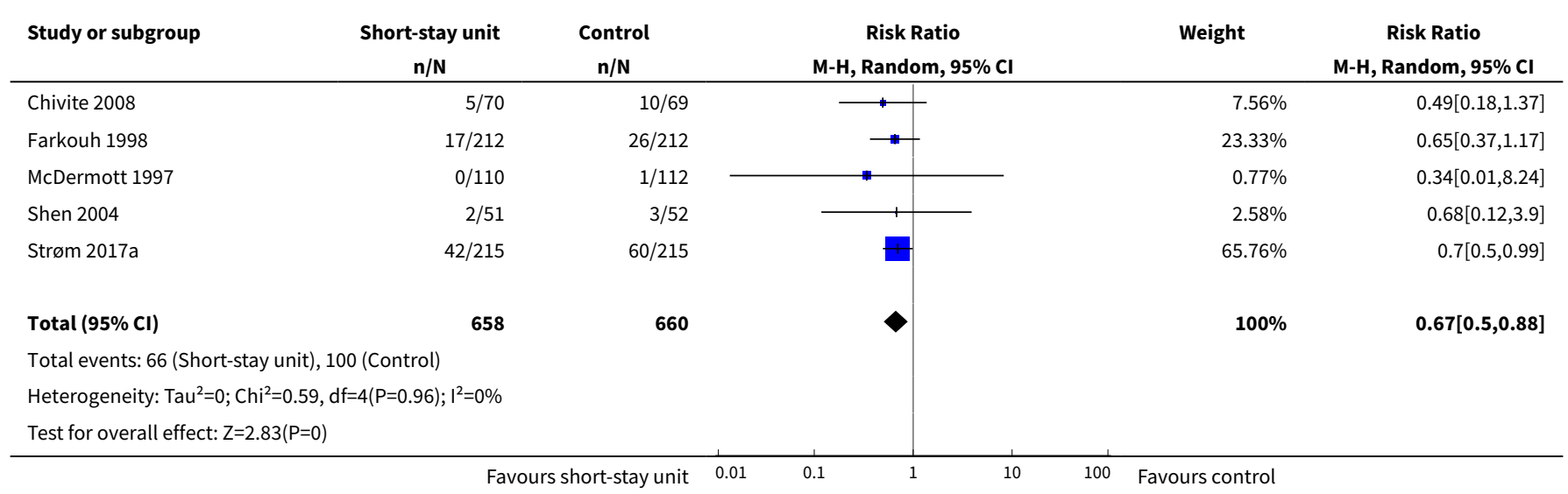


Analysis 1.11. Comparison 1 Mortality in participants treated in short-stay unit vs usual care, Outcome 11 Mortality at maximum follow-up worst-best case scenario.

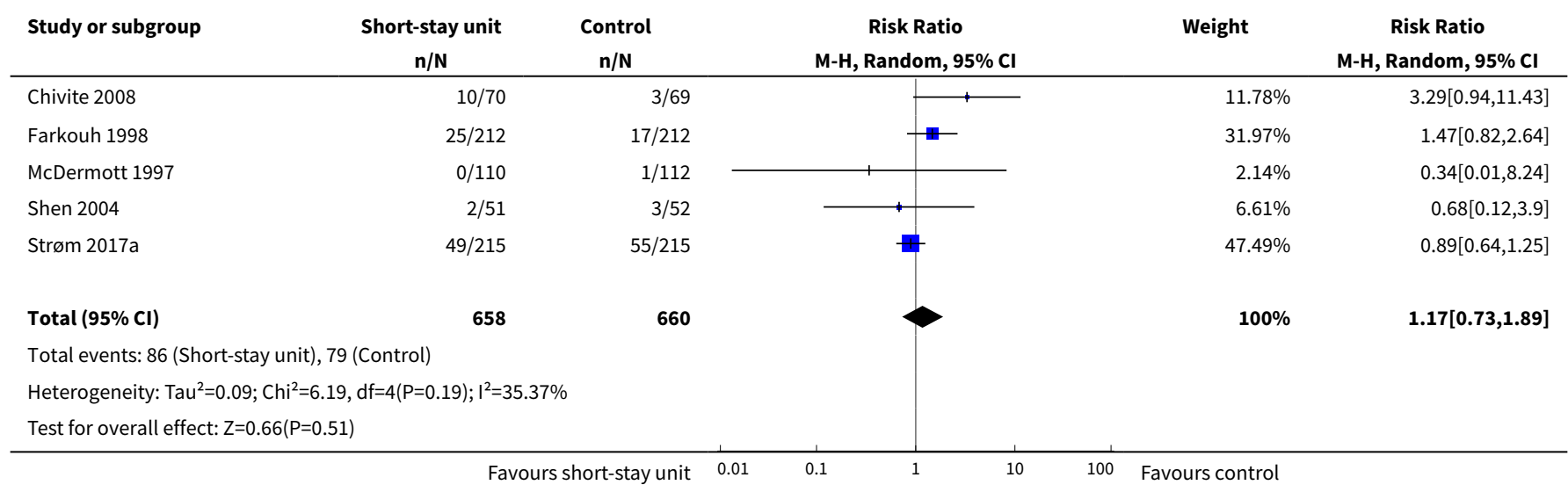

Analysis 1.12. Comparison 1 Mortality in participants treated in short-stay unit vs usual care, Outcome 12 Mortality at maximum follow-up published trials.

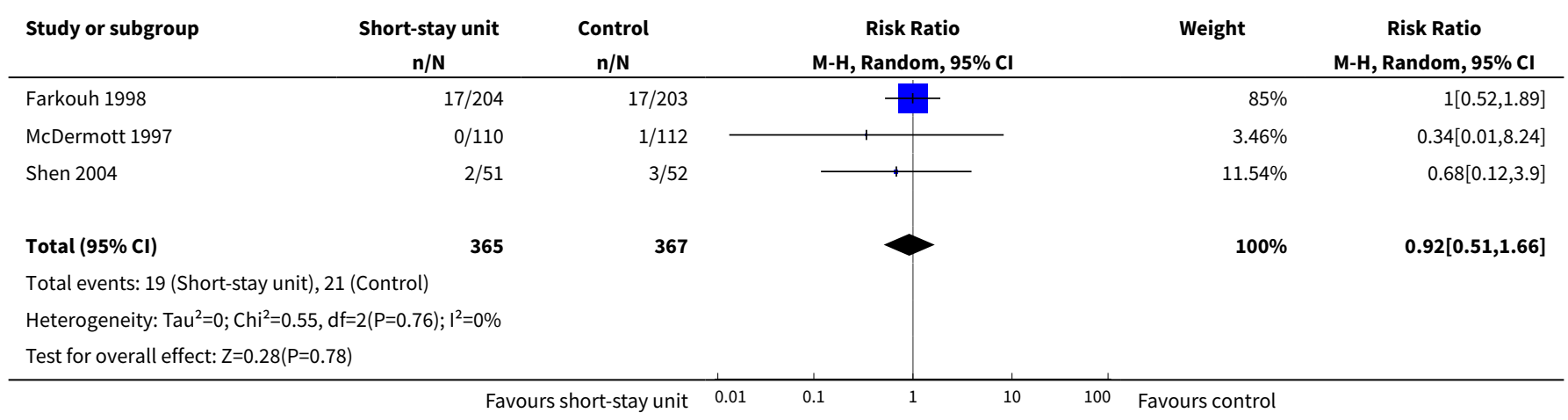

Analysis 1.13. Comparison 1 Mortality in participants treated in short-stay unit vs usual care, Outcome 13 Mortality at maximum follow-up and outcome assessed within 6 months of randomisation.

\begin{tabular}{|c|c|c|c|c|c|}
\hline Study or subgroup & $\begin{array}{c}\text { Short-stay unit } \\
n / N\end{array}$ & $\begin{array}{c}\text { Control } \\
\mathrm{n} / \mathrm{N}\end{array}$ & $\begin{array}{c}\text { Risk Ratio } \\
\text { M-H, Random, 95\% Cl }\end{array}$ & Weight & $\begin{array}{c}\text { Risk Ratio } \\
\text { M-H, Random, } 95 \% \mathrm{Cl}\end{array}$ \\
\hline Chivite 2008 & $5 / 65$ & $3 / 62$ & - & $4.69 \%$ & $1.59[0.4,6.37]$ \\
\hline Farkouh 1998 & $17 / 204$ & $17 / 203$ & - & $21.83 \%$ & $1[0.52,1.89]$ \\
\hline McDermott 1997 & $0 / 110$ & $1 / 112$ & & $0.89 \%$ & $0.34[0.01,8.24]$ \\
\hline Strøm 2017a & $42 / 208$ & $55 / 210$ & & $72.59 \%$ & $0.77[0.54,1.1]$ \\
\hline \multicolumn{6}{|c|}{ Total events: 64 (Short-stay unit), 76 (Control) } \\
\hline \multicolumn{6}{|c|}{ Heterogeneity: Tau $^{2}=0 ; \mathrm{Chi}^{2}=1.62, \mathrm{df}=3(\mathrm{P}=0.66) ; \mathrm{I}^{2}=0 \%$} \\
\hline Test for overall effec & & & & & \\
\hline
\end{tabular}


Analysis 1.14. Comparison 1 Mortality in participants treated in short-stay unit vs usual care, Outcome 14 Mortality at maximum follow-up multipurpose unit vs specialised unit.

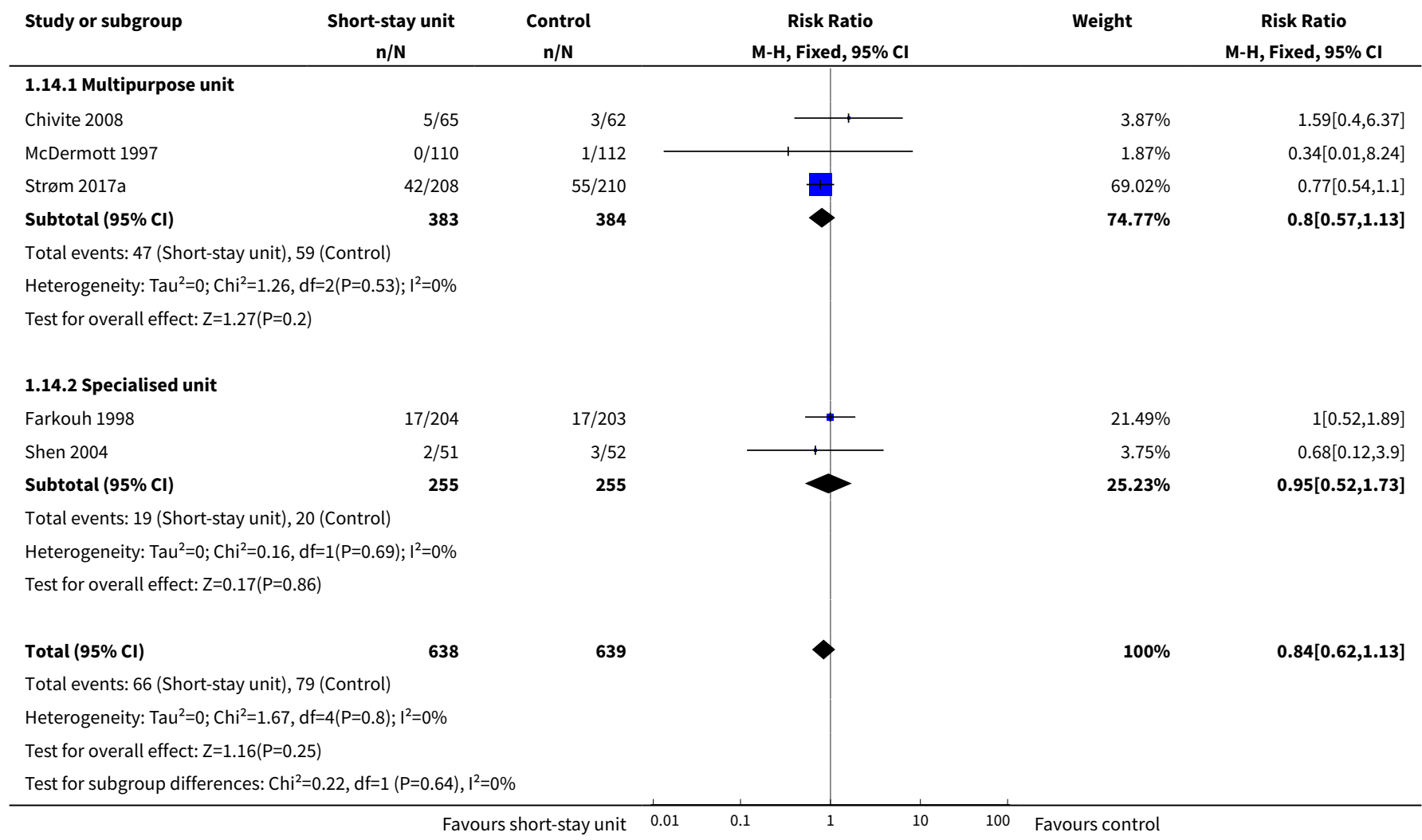

\section{Analysis 1.15. Comparison 1 Mortality in participants treated in short-stay unit vs usual care, Outcome 15 Mortality at maximum follow-up non-protocol-based vs protocol-based care.}

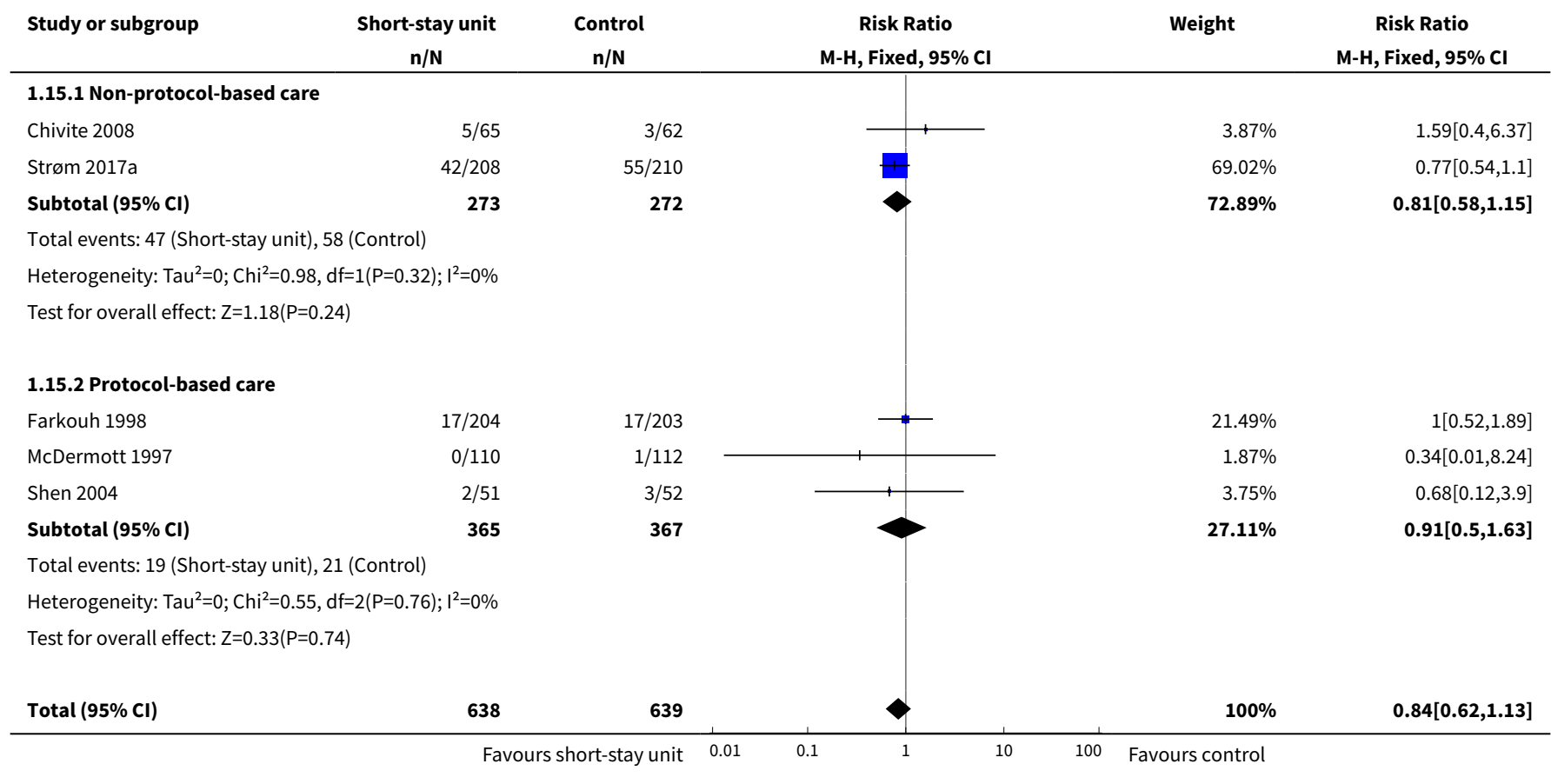




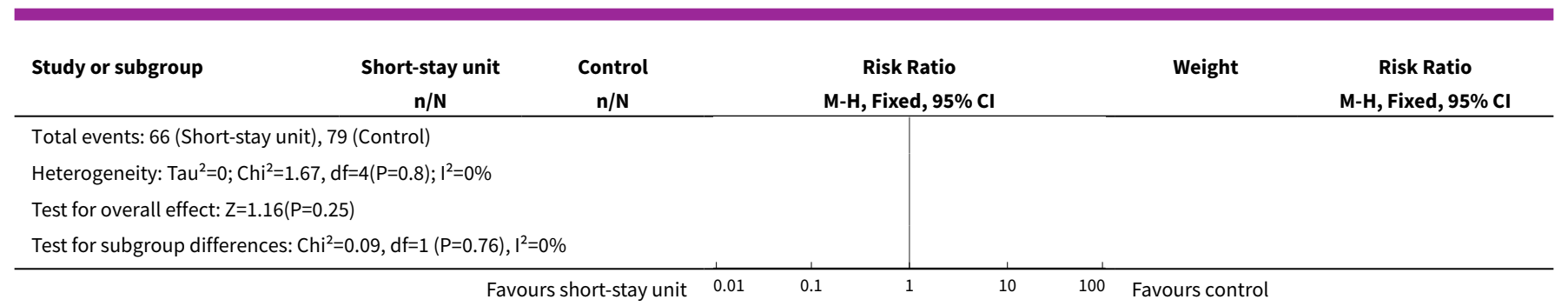

Analysis 1.16. Comparison 1 Mortality in participants treated in short-stay unit vs usual care, Outcome 16 Mortality at maximum follow-up older vs younger participants.

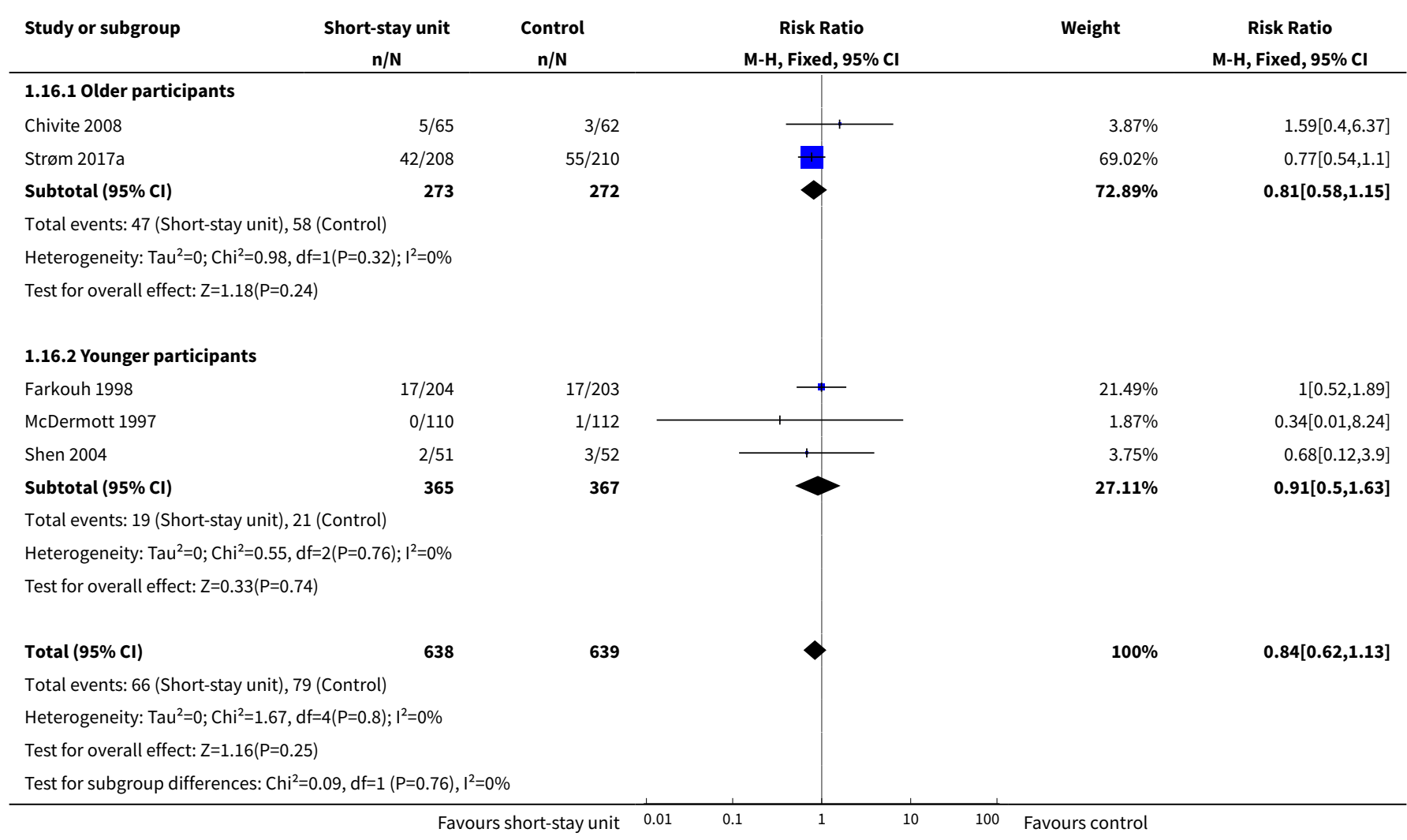

Comparison 2. Serious adverse events in participants treated in short-stay unit vs usual care

\begin{tabular}{lllll}
\hline Outcome or subgroup title & No. of studies & $\begin{array}{l}\text { No. of partici- } \\
\text { pants }\end{array}$ & Statistical method & Effect size \\
\hline $\begin{array}{l}\text { 1 Serious adverse events at time point } \\
\text { closest to 90-days }\end{array}$ & 7 & 1907 & $\begin{array}{l}\text { Risk Ratio (M-H, Random, } \\
95 \% \mathrm{Cl})\end{array}$ & $0.95[0.59,1.54]$ \\
\hline $\begin{array}{l}\text { 2 Serious adverse events at time point } \\
\text { closest to 90 days best-worst case scenario }\end{array}$ & 7 & 1929 & $\begin{array}{l}\text { Risk Ratio (M-H, Random, } \\
95 \% \mathrm{Cl})\end{array}$ \\
\hline $\begin{array}{l}\text { 3 Serious adverse events at time point } \\
\text { closest to 90 days worst-best case scenario }\end{array}$ & 7 & $10.790,1.26]$ \\
\hline
\end{tabular}




\begin{tabular}{|c|c|c|c|c|}
\hline Outcome or subgroup title & No. of studies & $\begin{array}{l}\text { No. of partici- } \\
\text { pants }\end{array}$ & Statistical method & Effect size \\
\hline $\begin{array}{l}4 \text { Serious adverse events at time point } \\
\text { closest to 90-days published trials }\end{array}$ & 6 & 1489 & $\begin{array}{l}\text { Risk Ratio (M-H, Random, } \\
95 \% \mathrm{Cl})\end{array}$ & $0.97[0.54,1.73]$ \\
\hline $\begin{array}{l}5 \text { Serious adverse events at time point } \\
\text { closest to } 90 \text {-days and outcome assessed } \\
\text { within } 6 \text { months of randomisation }\end{array}$ & 7 & 1907 & $\begin{array}{l}\text { Risk Ratio (M-H, Random, } \\
95 \% \mathrm{Cl})\end{array}$ & $0.95[0.59,1.54]$ \\
\hline $\begin{array}{l}6 \text { Serious adverse events at time point } \\
\text { closest to } 90 \text { days multipurpose unit vs } \\
\text { specialised unit }\end{array}$ & 7 & 1907 & $\begin{array}{l}\text { Risk Ratio (M-H, Random, } \\
95 \% \mathrm{Cl})\end{array}$ & $0.95[0.59,1.54]$ \\
\hline 6.1 multipurpose unit & 6 & 1483 & $\begin{array}{l}\text { Risk Ratio (M-H, Random, } \\
95 \% \mathrm{Cl})\end{array}$ & $1.23[0.88,1.72]$ \\
\hline 6.2 Specialised units & 1 & 424 & $\begin{array}{l}\text { Risk Ratio (M-H, Random, } \\
95 \% \mathrm{Cl})\end{array}$ & $0.47[0.21,1.07]$ \\
\hline $\begin{array}{l}7 \text { Serious adverse events at time point } \\
\text { closest to } 90 \text {-days non-protocol-based vs } \\
\text { protocol-based care }\end{array}$ & 7 & 1907 & $\begin{array}{l}\text { Risk Ratio (M-H, Random, } \\
95 \% \mathrm{Cl})\end{array}$ & $0.95[0.59,1.54]$ \\
\hline 7.1 Non protocol-based care & 1 & 418 & $\begin{array}{l}\text { Risk Ratio (M-H, Random, } \\
95 \% \mathrm{Cl})\end{array}$ & $0.79[0.30,2.07]$ \\
\hline 7.2 Protocol-based care & 6 & 1489 & $\begin{array}{l}\text { Risk Ratio (M-H, Random, } \\
95 \% \mathrm{Cl})\end{array}$ & $0.97[0.54,1.73]$ \\
\hline $\begin{array}{l}8 \text { Serious adverse events at time point } \\
\text { closest to 90-days older vs younger partici- } \\
\text { pants }\end{array}$ & 7 & 1907 & $\begin{array}{l}\text { Risk Ratio (M-H, Random, } \\
95 \% \mathrm{Cl})\end{array}$ & $0.95[0.59,1.54]$ \\
\hline 8.1 Older participants & 1 & 418 & $\begin{array}{l}\text { Risk Ratio (M-H, Random, } \\
95 \% \mathrm{Cl})\end{array}$ & $0.79[0.30,2.07]$ \\
\hline 8.2 Younger participants & 6 & 1489 & $\begin{array}{l}\text { Risk Ratio (M-H, Random, } \\
95 \% \mathrm{Cl})\end{array}$ & $0.97[0.54,1.73]$ \\
\hline $\begin{array}{l}9 \text { Serious adverse events at maximum fol- } \\
\text { low-up }\end{array}$ & 8 & 1988 & $\begin{array}{l}\text { Risk Ratio (M-H, Random, } \\
95 \% \mathrm{Cl})\end{array}$ & $1.11[0.87,1.41]$ \\
\hline $\begin{array}{l}10 \text { Serious adverse events multipurpose } \\
\text { unit at maximum follow-up best-worst } \\
\text { case scenario }\end{array}$ & 8 & 2039 & $\begin{array}{l}\text { Risk Ratio (M-H, Random, } \\
95 \% \mathrm{Cl})\end{array}$ & $0.83[0.60,1.16]$ \\
\hline $\begin{array}{l}11 \text { Serious adverse events multipurpose } \\
\text { unit at maximum follow-up worst-best } \\
\text { case scenario }\end{array}$ & 8 & 2039 & $\begin{array}{l}\text { Risk Ratio (M-H, Random, } \\
95 \% \mathrm{Cl})\end{array}$ & $1.35[1.07,1.70]$ \\
\hline $\begin{array}{l}12 \text { Serious adverse events multipurpose } \\
\text { unit at maximum follow-up published tri- } \\
\text { als }\end{array}$ & 7 & 1570 & $\begin{array}{l}\text { Risk Ratio (M-H, Random, } \\
95 \% \mathrm{Cl})\end{array}$ & $1.13[0.88,1.46]$ \\
\hline $\begin{array}{l}13 \text { Serious adverse events multipurpose } \\
\text { unit at maximum follow-up and outcome }\end{array}$ & 8 & 1988 & $\begin{array}{l}\text { Risk Ratio (M-H, Random, } \\
95 \% \mathrm{Cl} \text { ) }\end{array}$ & $1.11[0.87,1.41]$ \\
\hline
\end{tabular}




\begin{tabular}{|c|c|c|c|c|}
\hline Outcome or subgroup title & No. of studies & $\begin{array}{l}\text { No. of partici- } \\
\text { pants }\end{array}$ & Statistical method & Effect size \\
\hline \multicolumn{5}{|l|}{$\begin{array}{l}\text { assessed within } 6 \text { months of randomisa- } \\
\text { tion }\end{array}$} \\
\hline $\begin{array}{l}14 \text { Serious adverse events at maximum fol- } \\
\text { low-up multipurpose unit vs specialised } \\
\text { unit }\end{array}$ & 8 & 1988 & $\begin{array}{l}\text { Risk Ratio (M-H, Random, } \\
95 \% \mathrm{Cl})\end{array}$ & $1.11[0.87,1.41]$ \\
\hline 14.1 Multipurpose unit & 7 & 1581 & $\begin{array}{l}\text { Risk Ratio (M-H, Random, } \\
95 \% \mathrm{Cl})\end{array}$ & $1.14[0.83,1.56]$ \\
\hline 14.2 Specialised unit & 1 & 407 & $\begin{array}{l}\text { Risk Ratio (M-H, Random, } \\
95 \% \mathrm{Cl})\end{array}$ & $1.07[0.73,1.56]$ \\
\hline $\begin{array}{l}15 \text { Serious adverse events at maximum } \\
\text { follow-up non-protocol-based vs proto- } \\
\text { col-based care }\end{array}$ & 8 & 1988 & $\begin{array}{l}\text { Risk Ratio (M-H, Random, } \\
95 \% \mathrm{Cl})\end{array}$ & $1.11[0.87,1.41]$ \\
\hline 15.1 Non-protocol-based care & 1 & 418 & $\begin{array}{l}\text { Risk Ratio (M-H, Random, } \\
95 \% \mathrm{Cl})\end{array}$ & $0.79[0.30,2.07]$ \\
\hline 15.2 Protocol-based care & 7 & 1570 & $\begin{array}{l}\text { Risk Ratio (M-H, Random, } \\
95 \% \mathrm{Cl})\end{array}$ & $1.13[0.88,1.46]$ \\
\hline $\begin{array}{l}16 \text { Serious adverse events at maximum fol- } \\
\text { low-up older participants vs younger par- } \\
\text { ticipants }\end{array}$ & 8 & 1988 & $\begin{array}{l}\text { Risk Ratio (M-H, Random, } \\
95 \% \mathrm{Cl})\end{array}$ & $1.11[0.87,1.41]$ \\
\hline 16.1 Older participants & 1 & 418 & $\begin{array}{l}\text { Risk Ratio (M-H, Random, } \\
95 \% \mathrm{Cl})\end{array}$ & $0.79[0.30,2.07]$ \\
\hline 16.2 Younger participants & 7 & 1570 & $\begin{array}{l}\text { Risk Ratio (M-H, Random, } \\
95 \% \mathrm{Cl})\end{array}$ & $1.13[0.88,1.46]$ \\
\hline $\begin{array}{l}17 \text { Serious adverse events at time point } \\
\text { closest to } 90 \text { days }\end{array}$ & 7 & 1907 & $\begin{array}{l}\text { Risk Ratio (M-H, Random, } \\
95 \% \mathrm{Cl})\end{array}$ & $0.95[0.59,1.54]$ \\
\hline $\begin{array}{l}18 \text { Serious adverse events at time point } \\
\text { closest to } 90 \text { days w/o Farkouh } 1998\end{array}$ & 6 & 1483 & $\begin{array}{l}\text { Risk Ratio (M-H, Random, } \\
95 \% \mathrm{Cl} \text { ) }\end{array}$ & $1.23[0.88,1.72]$ \\
\hline
\end{tabular}

\section{Analysis 2.1. Comparison 2 Serious adverse events in participants treated in short-stay unit vs usual care, Outcome 1 Serious adverse events at time point closest to 90 -days.}

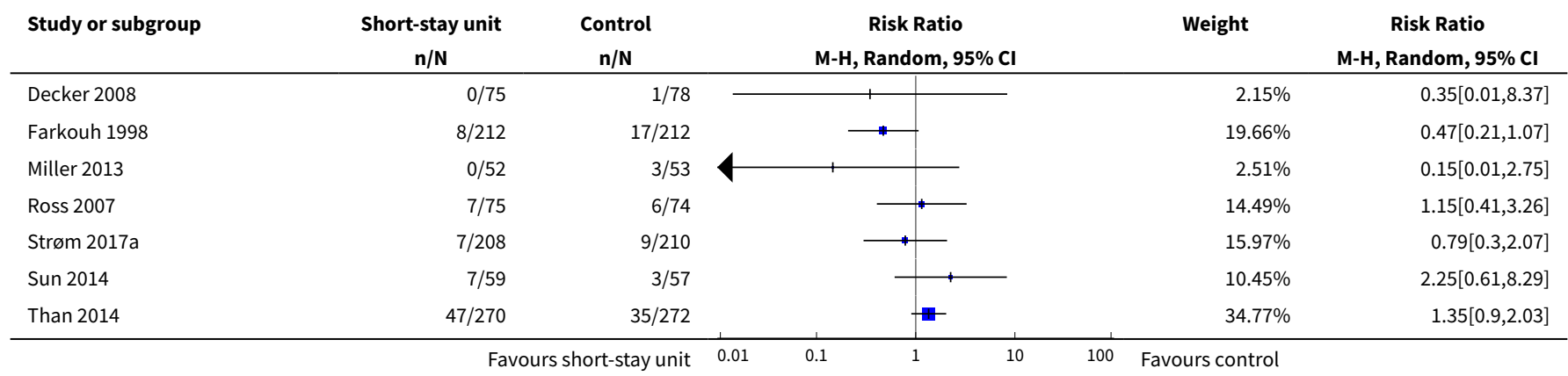




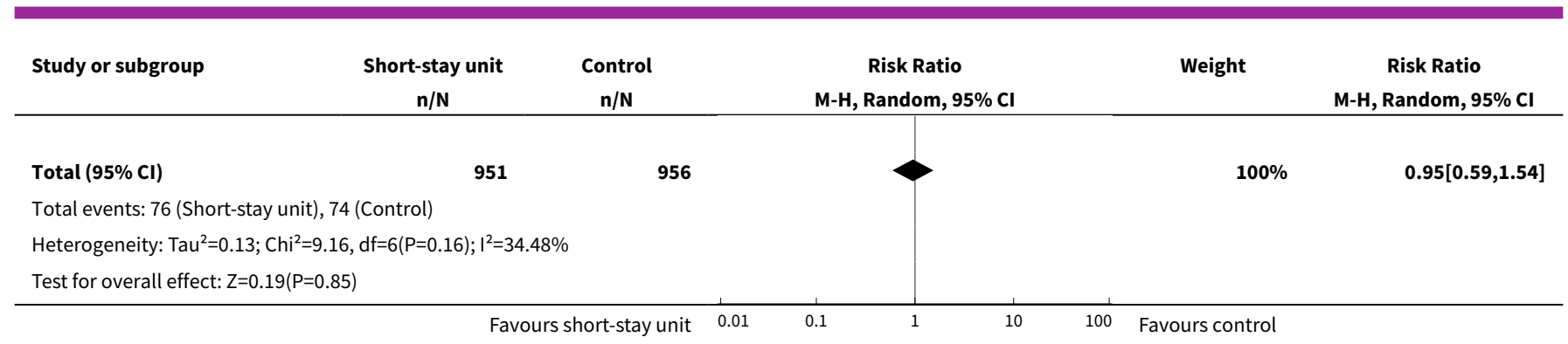

\section{Analysis 2.2. Comparison 2 Serious adverse events in participants treated in short-stay unit vs usual} care, Outcome 2 Serious adverse events at time point closest to 90 days best-worst case scenario.

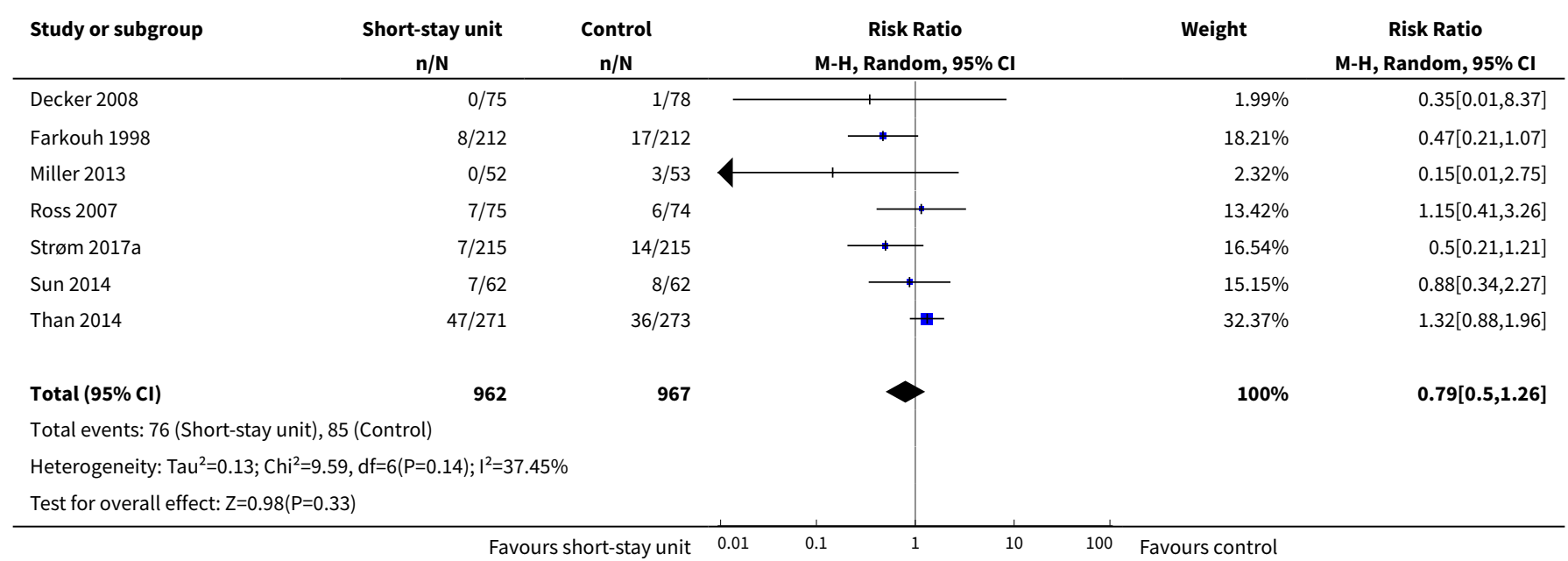

\section{Analysis 2.3. Comparison 2 Serious adverse events in participants treated in short-stay unit vs usual} care, Outcome 3 Serious adverse events at time point closest to 90 days worst-best case scenario.

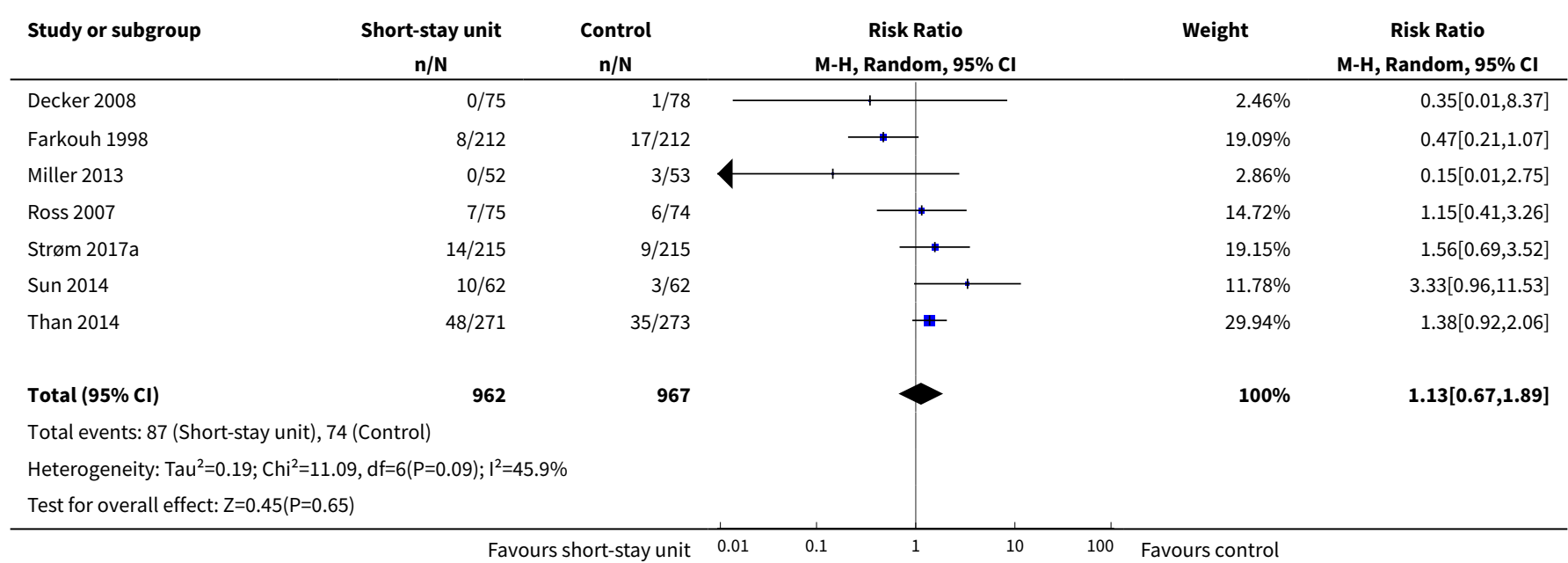


Analysis 2.4. Comparison 2 Serious adverse events in participants treated in short-stay unit vs usual care, Outcome 4 Serious adverse events at time point closest to 90 -days published trials.

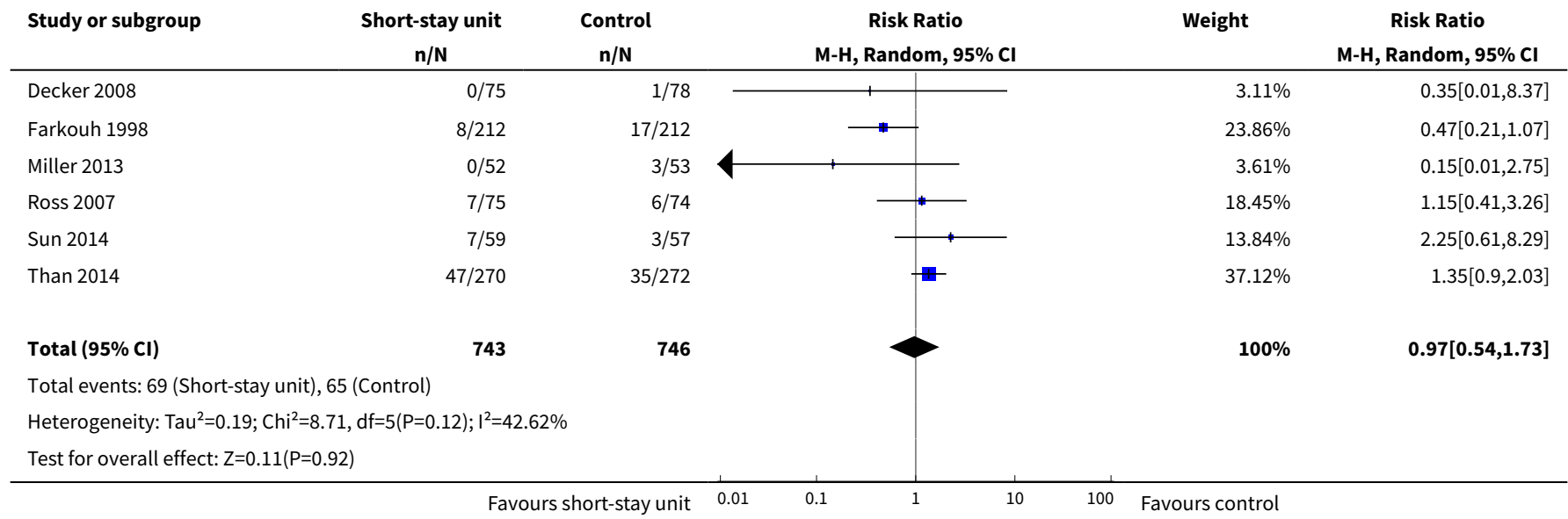

Analysis 2.5. Comparison 2 Serious adverse events in participants treated in short-stay unit vs usual care, Outcome 5 Serious adverse events at time point closest to 90-days and outcome assessed within 6 months of randomisation.

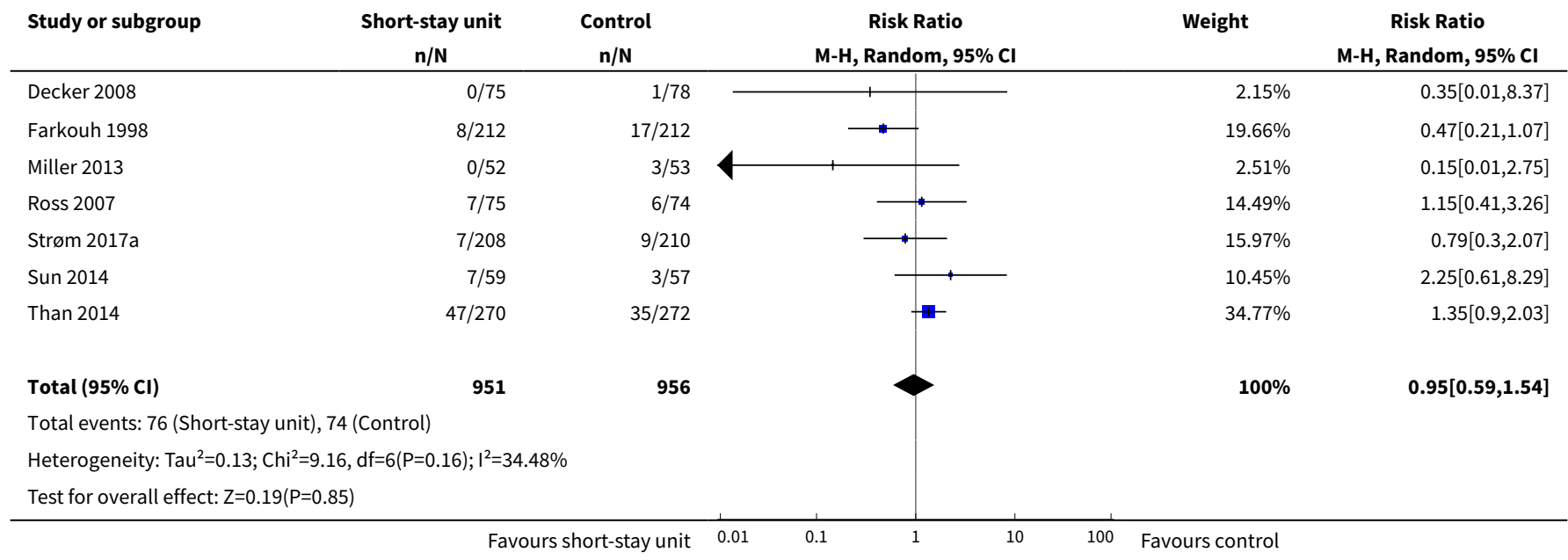

Analysis 2.6. Comparison 2 Serious adverse events in participants treated in short-stay unit vs usual care, Outcome 6 Serious adverse events at time point closest to 90 days multipurpose unit vs specialised unit.

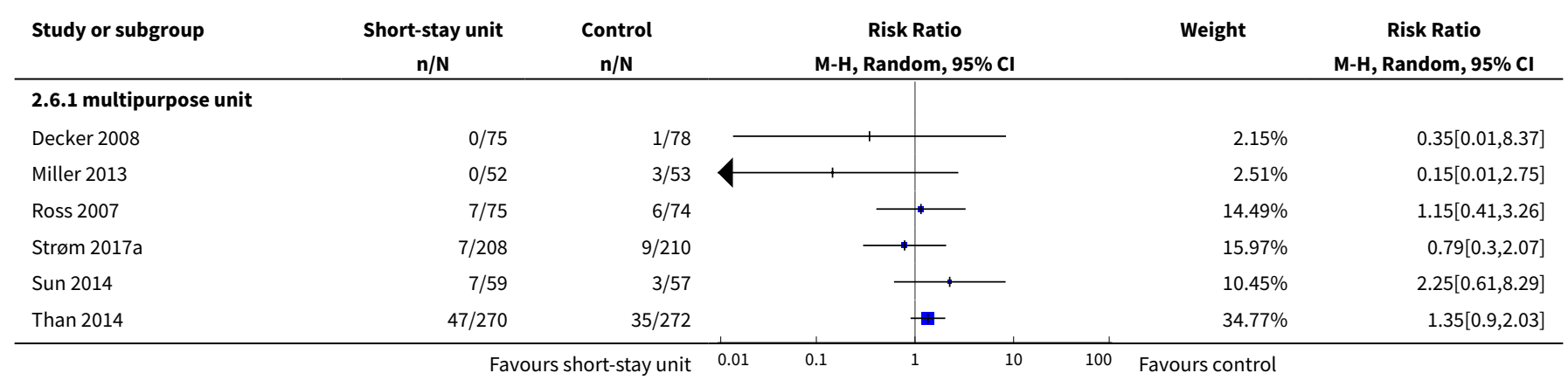




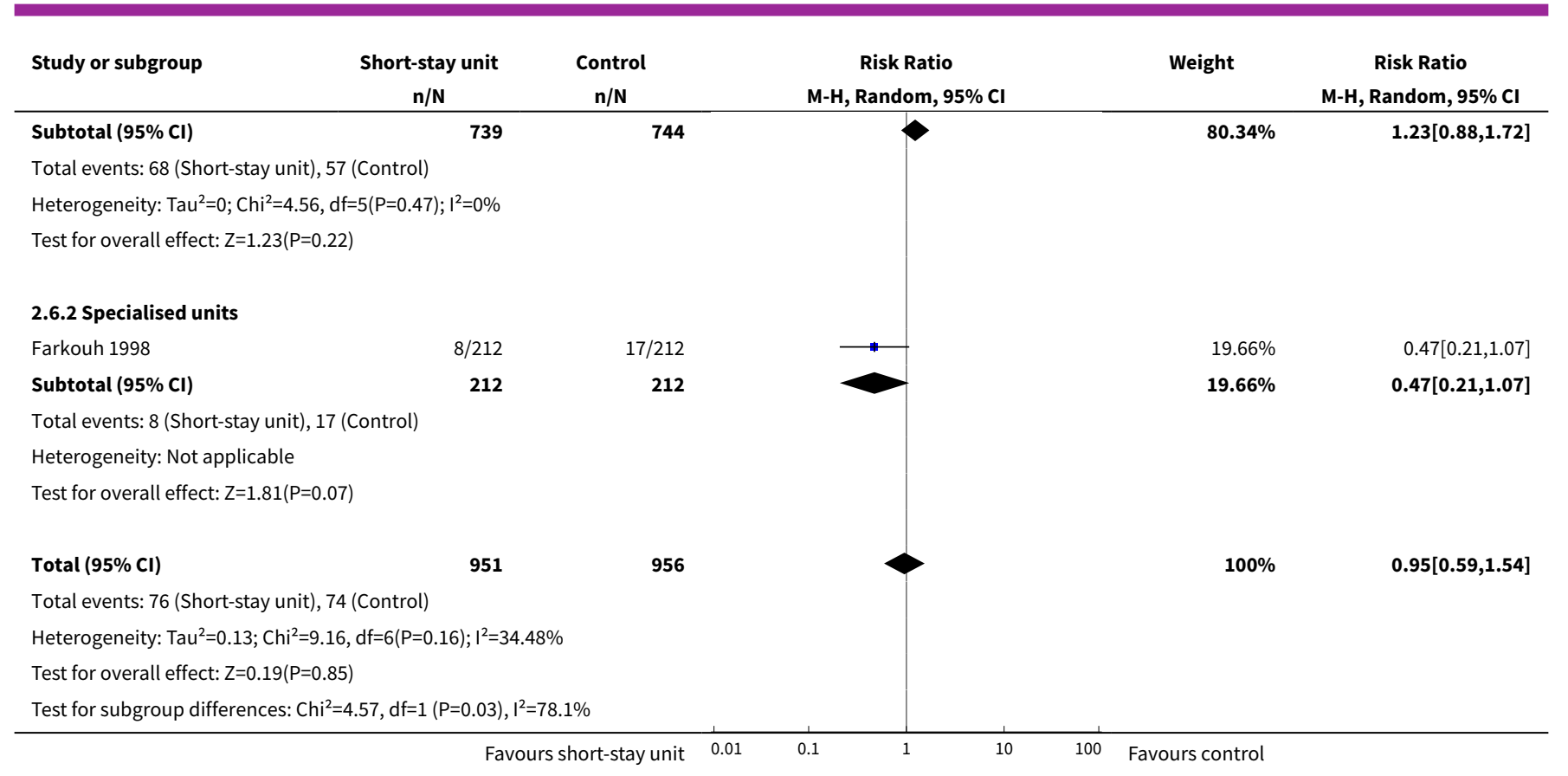

\section{Analysis 2.7. Comparison 2 Serious adverse events in participants treated in short-stay unit vs usual care, Outcome 7 Serious adverse events at time point closest to 90 -days non-protocol-based vs protocol-based care.}

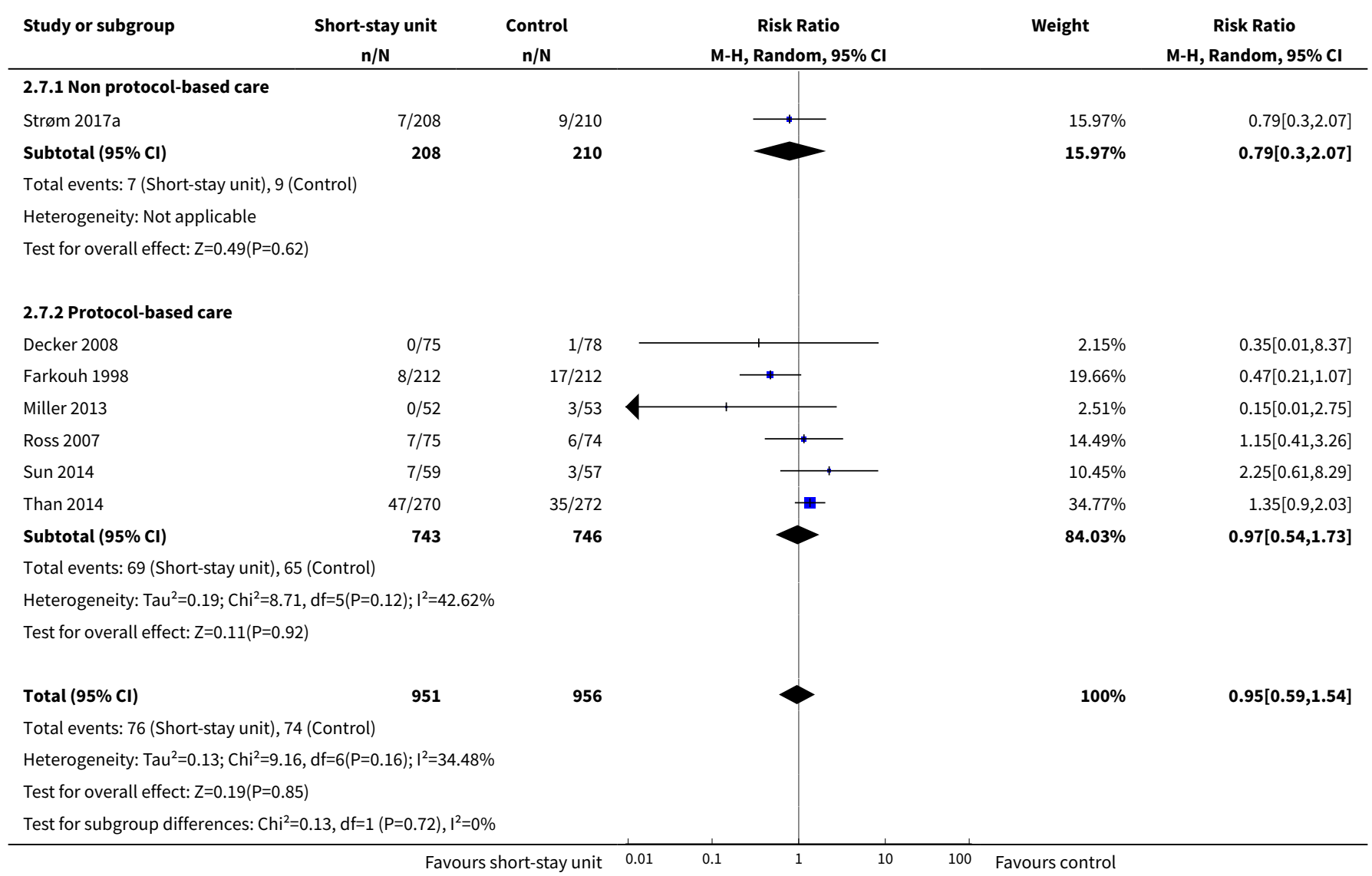


Analysis 2.8. Comparison 2 Serious adverse events in participants treated in short-stay unit vs usual care, Outcome 8 Serious adverse events at time point closest to 90 -days older vs younger participants.

\begin{tabular}{|c|c|c|c|c|c|}
\hline Study or subgroup & $\begin{array}{c}\text { Short-stay unit } \\
n / N \\
\end{array}$ & $\begin{array}{c}\text { Control } \\
\mathrm{n} / \mathrm{N}\end{array}$ & $\begin{array}{c}\text { Risk Ratio } \\
\text { M-H, Random, } 95 \% \text { CI }\end{array}$ & Weight & $\begin{array}{c}\text { Risk Ratio } \\
\text { M-H, Random, } 95 \% \mathrm{Cl}\end{array}$ \\
\hline \multicolumn{6}{|c|}{ 2.8.1 Older participants } \\
\hline Strøm 2017a & $7 / 208$ & $9 / 210$ & $\longrightarrow$ & $15.97 \%$ & $0.79[0.3,2.07]$ \\
\hline Subtotal $(95 \% \mathrm{Cl})$ & 208 & 210 & & $15.97 \%$ & $0.79[0.3,2.07]$ \\
\hline \multicolumn{6}{|c|}{ Total events: 7 (Short-stay unit), 9 (Control) } \\
\hline \multicolumn{6}{|c|}{ Heterogeneity: Not applicable } \\
\hline \multicolumn{6}{|c|}{ 2.8.2 Younger participants } \\
\hline Decker 2008 & $0 / 75$ & $1 / 78$ & & $2.15 \%$ & $0.35[0.01,8.37]$ \\
\hline Farkouh 1998 & $8 / 212$ & $17 / 212$ & & $19.66 \%$ & $0.47[0.21,1.07]$ \\
\hline Miller 2013 & $0 / 52$ & $3 / 53$ & & $2.51 \%$ & $0.15[0.01,2.75]$ \\
\hline Ross 2007 & $7 / 75$ & $6 / 74$ & - & $14.49 \%$ & $1.15[0.41,3.26]$ \\
\hline Than 2014 & $47 / 270$ & $35 / 272$ & - & $34.77 \%$ & $1.35[0.9,2.03]$ \\
\hline Subtotal $(95 \% \mathrm{Cl})$ & 743 & 746 & & $84.03 \%$ & $0.97[0.54,1.73]$ \\
\hline \multicolumn{6}{|c|}{ Total events: 69 (Short-stay unit), 65 (Control) } \\
\hline \multicolumn{6}{|c|}{ Heterogeneity: Tau $^{2}=0.19 ; \mathrm{Chi}^{2}=8.71, \mathrm{df}=5(\mathrm{P}=0.12) ; \mathrm{I}^{2}=42.62 \%$} \\
\hline \multicolumn{6}{|c|}{ Test for overall effect: $\mathrm{Z}=0.11(\mathrm{P}=0.92)$} \\
\hline Total $(95 \% \mathrm{Cl})$ & 951 & 956 & & $100 \%$ & $0.95[0.59,1.54]$ \\
\hline \multicolumn{6}{|c|}{ Total events: 76 (Short-stay unit), 74 (Control) } \\
\hline \multicolumn{6}{|c|}{ Heterogeneity: $\operatorname{Tau}^{2}=0.13 ; \mathrm{Chi}^{2}=9.16, \mathrm{df}=6(\mathrm{P}=0.16) ; \mathrm{I}^{2}=34.48 \%$} \\
\hline \multicolumn{6}{|c|}{ Test for overall effect: $Z=0.19(P=0.85)$} \\
\hline Test for subgroup dif & $.13, d f=1(P=0.72)$, & & & & \\
\hline
\end{tabular}

Analysis 2.9. Comparison 2 Serious adverse events in participants treated in shortstay unit vs usual care, Outcome 9 Serious adverse events at maximum follow-up.

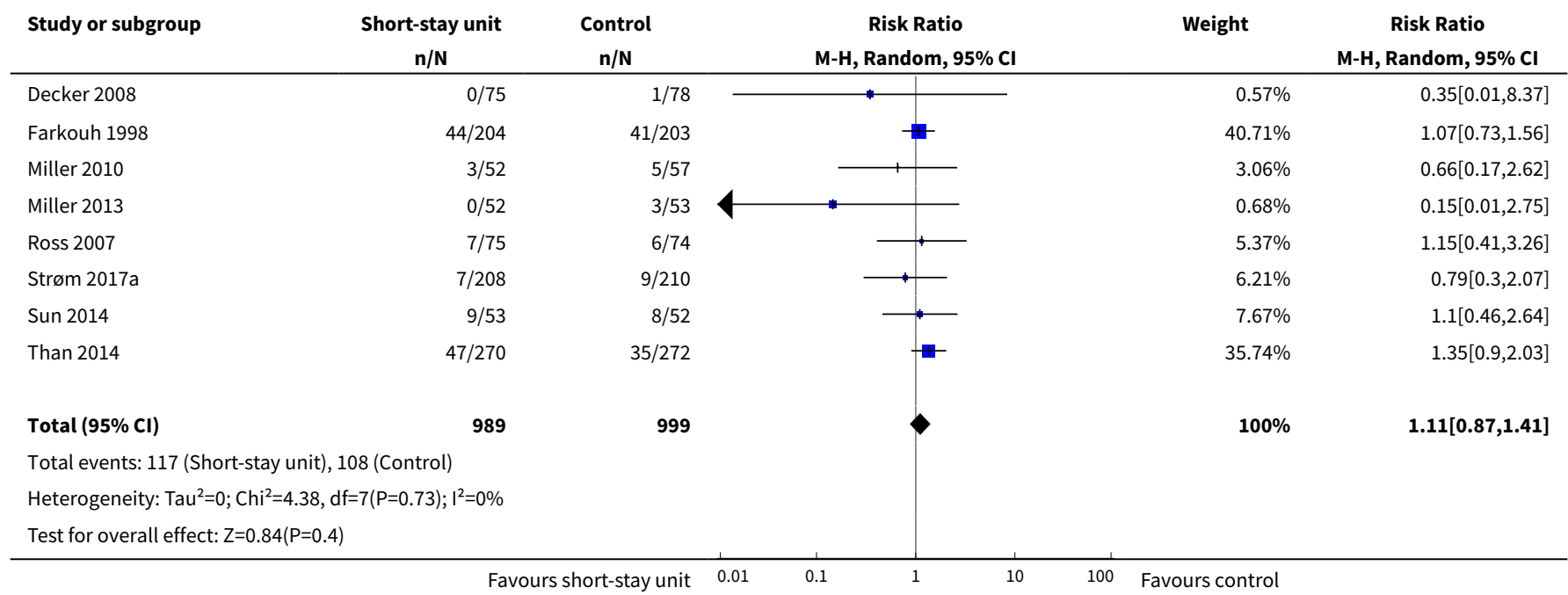


Analysis 2.10. Comparison 2 Serious adverse events in participants treated in short-stay unit vs usual care, Outcome 10 Serious adverse events multipurpose unit at maximum follow-up best-worst case scenario.

\begin{tabular}{|c|c|c|c|c|c|}
\hline Study or subgroup & $\begin{array}{c}\text { Short-stay unit } \\
n / N\end{array}$ & $\begin{array}{c}\text { Control } \\
n / N\end{array}$ & $\begin{array}{c}\text { Risk Ratio } \\
\text { M-H, Random, 95\% Cl }\end{array}$ & Weight & $\begin{array}{c}\text { Risk Ratio } \\
\text { M-H, Random, 95\% Cl }\end{array}$ \\
\hline Decker 2008 & $0 / 75$ & $1 / 78$ & 1 & $1.05 \%$ & $0.35[0.01,8.37]$ \\
\hline Farkouh 1998 & $44 / 212$ & $50 / 212$ & $\rightarrow$ & $30.74 \%$ & $0.88[0.62,1.26]$ \\
\hline Miller 2010 & $3 / 53$ & $5 / 57$ & & $5.1 \%$ & $0.65[0.16,2.57]$ \\
\hline Miller 2013 & $0 / 52$ & $3 / 53$ & & $1.23 \%$ & $0.15[0.01,2.75]$ \\
\hline Ross 2007 & $7 / 75$ & $6 / 74$ & - & $8.3 \%$ & $1.15[0.41,3.26]$ \\
\hline Sun 2014 & $9 / 62$ & $18 / 62$ & $\longrightarrow$ & $14.66 \%$ & $0.5[0.24,1.03]$ \\
\hline Than 2014 & $47 / 271$ & $36 / 273$ & - & $28.17 \%$ & $1.32[0.88,1.96]$ \\
\hline Total $(95 \% \mathrm{Cl})$ & 1015 & 1024 & & $100 \%$ & $0.83[0.6,1.16]$ \\
\hline \multicolumn{6}{|c|}{ Total events: 117 (Short-stay unit), 133 (Control) } \\
\hline \multicolumn{6}{|c|}{ Heterogeneity: $\mathrm{Tau}^{2}=0.06 ; \mathrm{Chi}^{2}=10.01, \mathrm{df}=7(\mathrm{P}=0.19) ; \mathrm{I}^{2}=30.06 \%$} \\
\hline
\end{tabular}

\section{Analysis 2.11. Comparison 2 Serious adverse events in participants treated in short-stay unit vs usual care, Outcome 11 Serious adverse events multipurpose unit at maximum follow-up worst-best case scenario.}

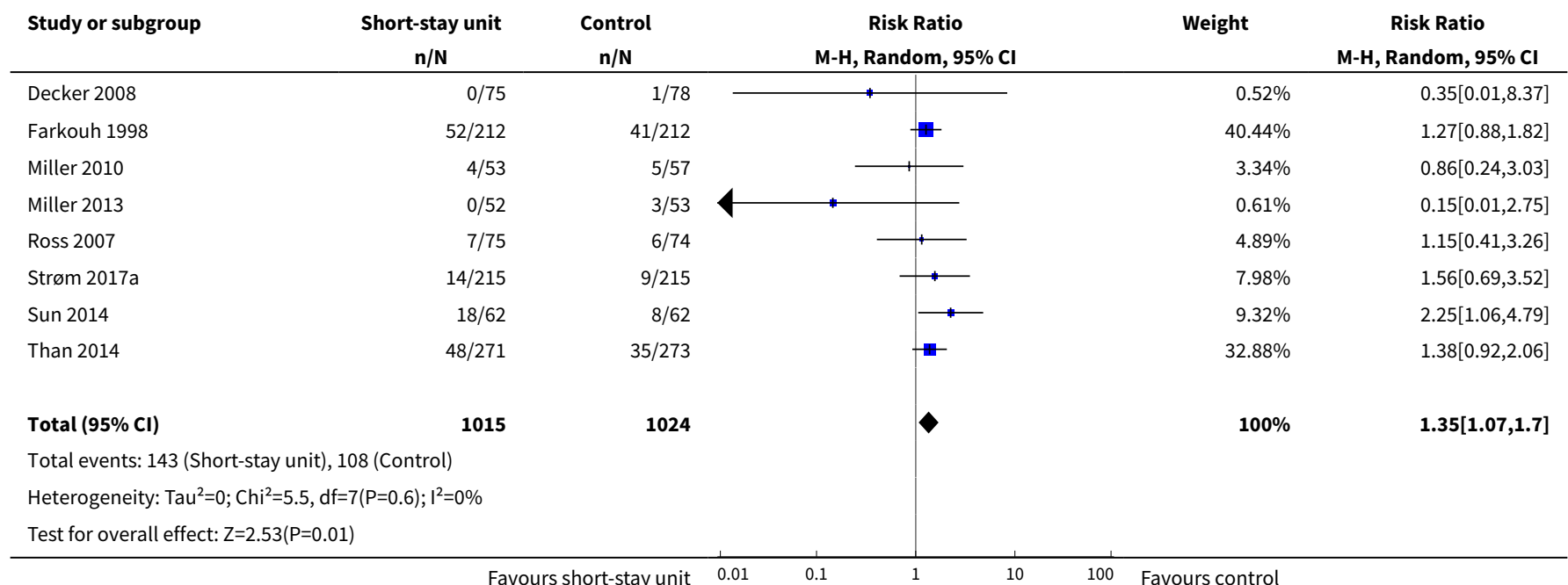

Analysis 2.12. Comparison 2 Serious adverse events in participants treated in short-stay unit vs usual care, Outcome 12 Serious adverse events multipurpose unit at maximum follow-up published trials.

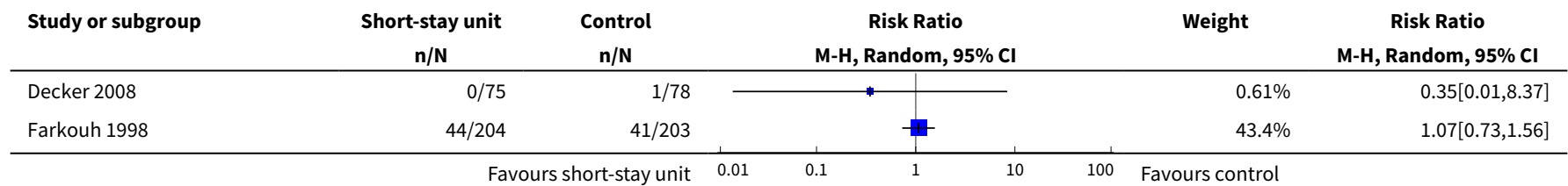




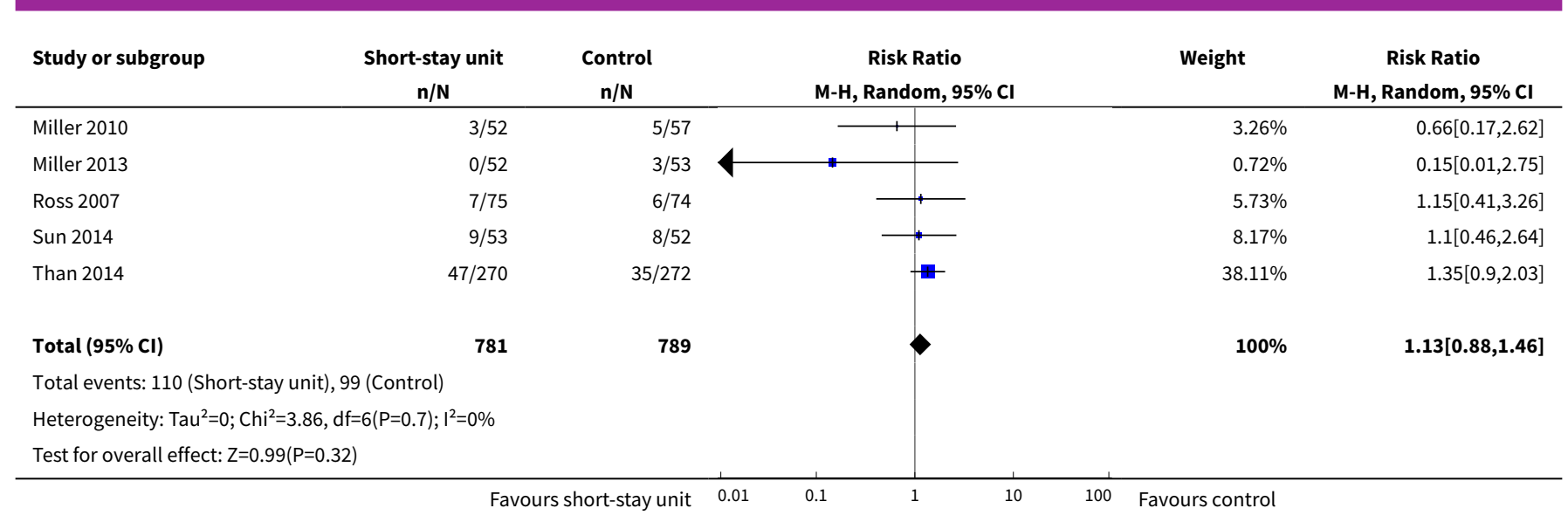

\section{Analysis 2.13. Comparison 2 Serious adverse events in participants treated in short- stay unit vs usual care, Outcome 13 Serious adverse events multipurpose unit at maximum follow-up and outcome assessed within 6 months of randomisation.}

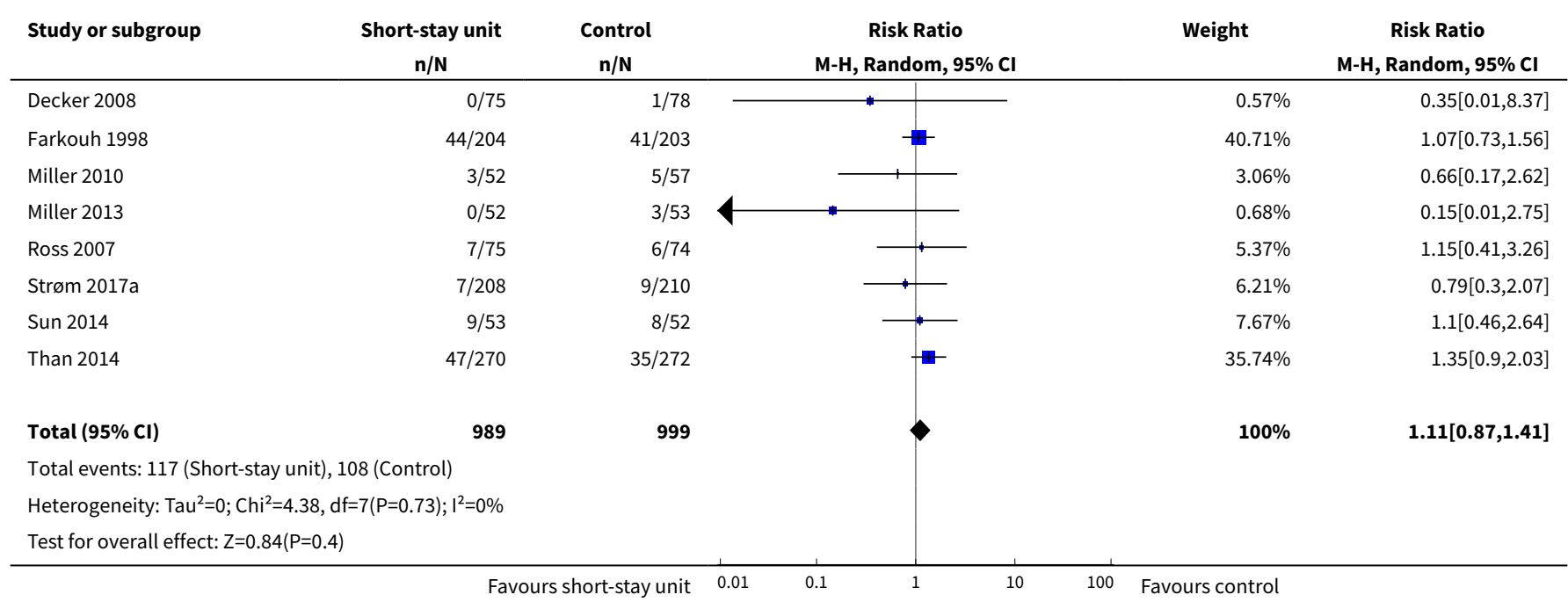

Analysis 2.14. Comparison 2 Serious adverse events in participants treated in short-stay unit vs usual care, Outcome 14 Serious adverse events at maximum follow-up multipurpose unit vs specialised unit.

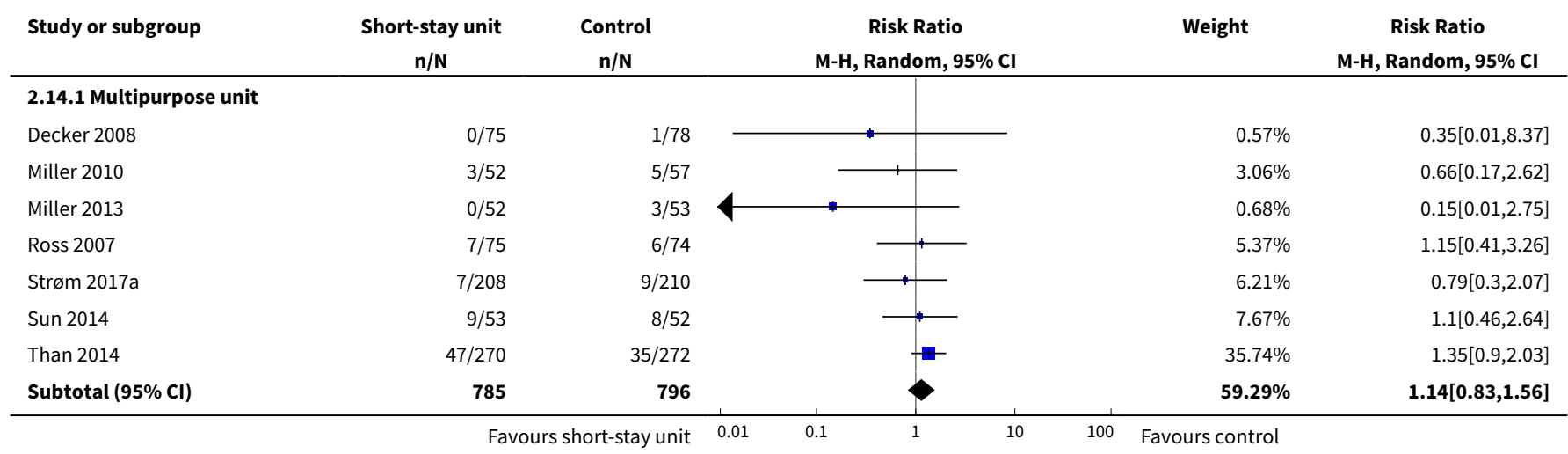




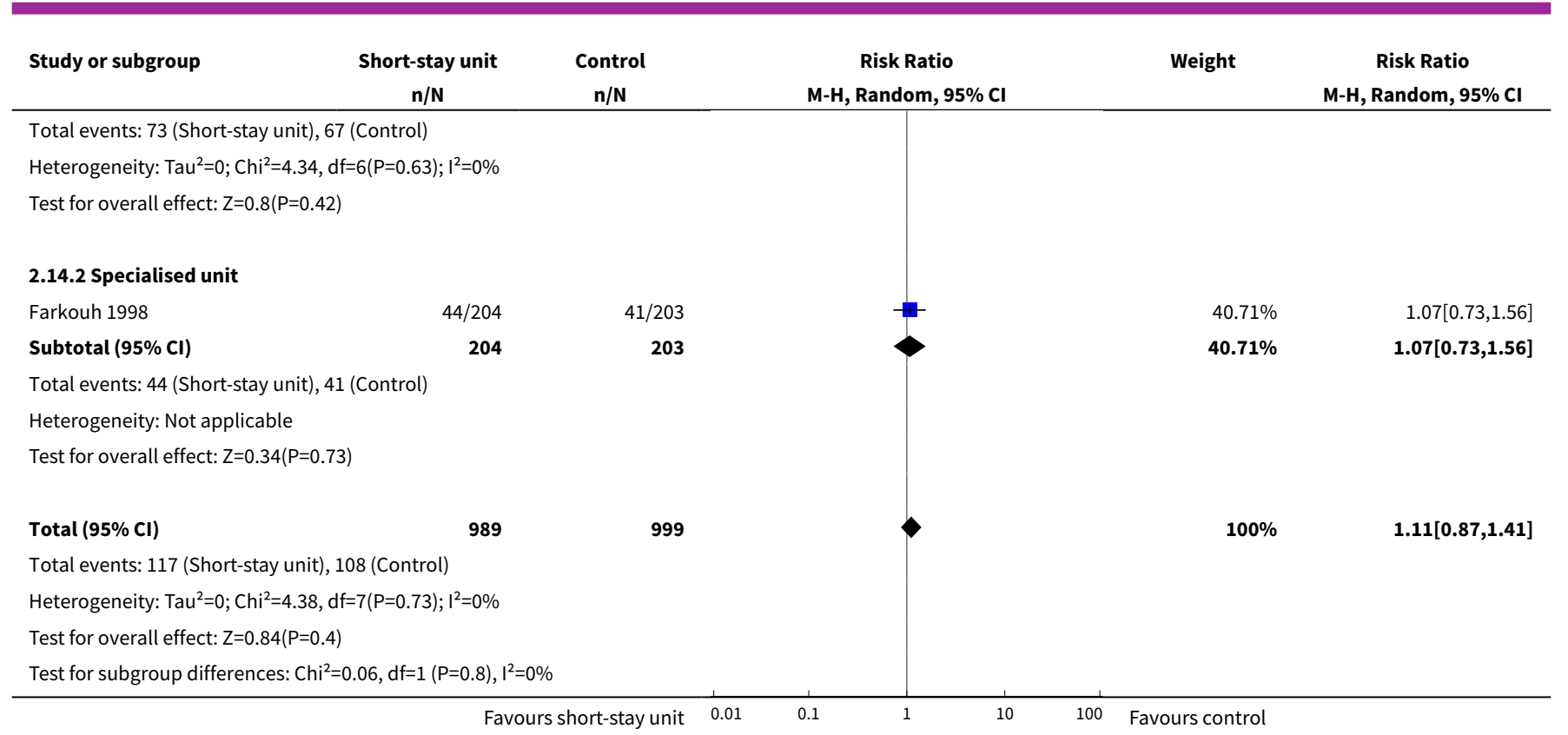

\section{Analysis 2.15. Comparison 2 Serious adverse events in participants treated in short-stay unit vs usual care, Outcome 15 Serious adverse events at maximum follow-up non-protocol-based vs protocol-based care.}

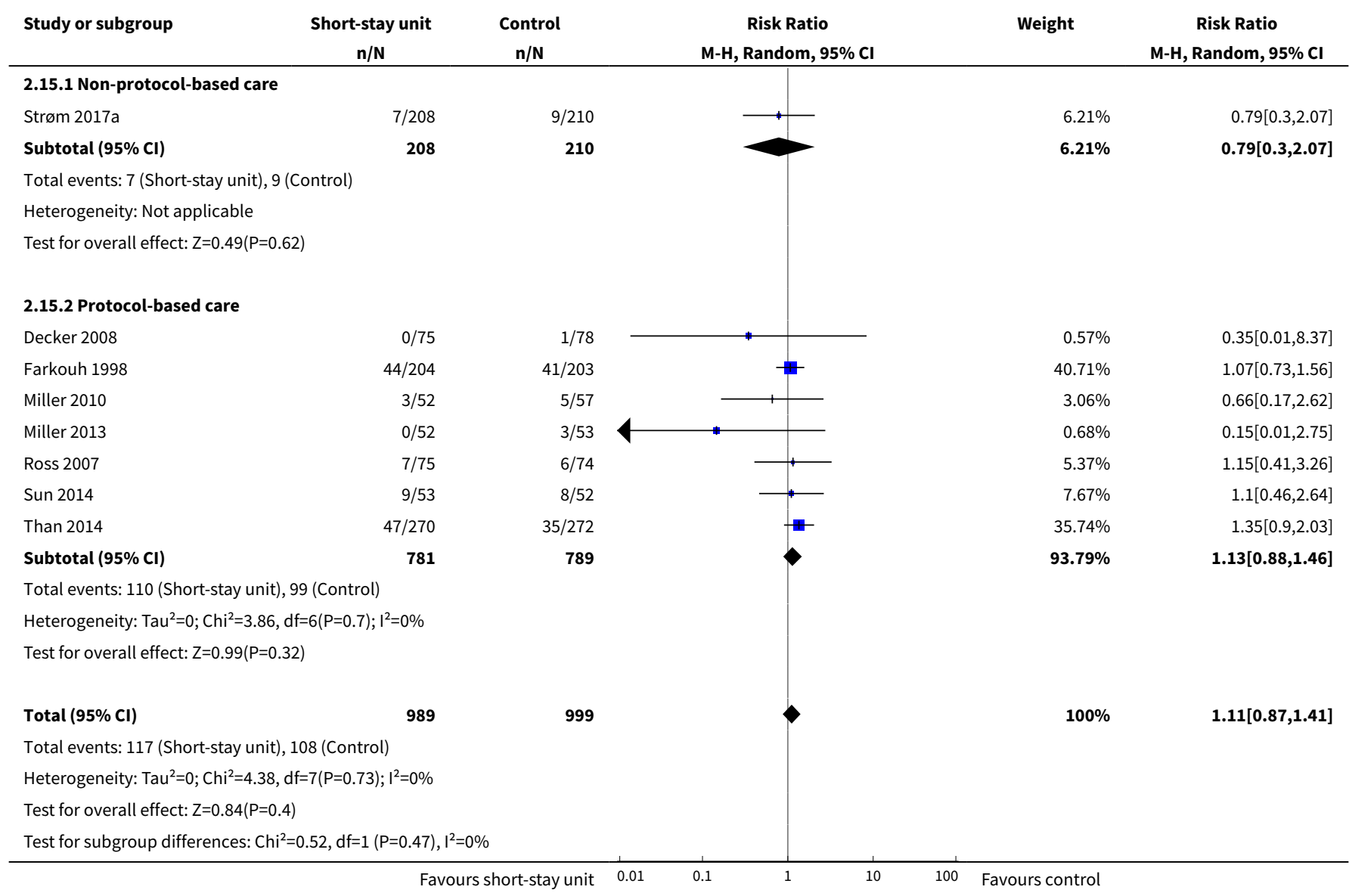


Analysis 2.16. Comparison 2 Serious adverse events in participants treated in short-stay unit vs usual care, Outcome 16 Serious adverse events at maximum follow-up older participants vs younger participants.

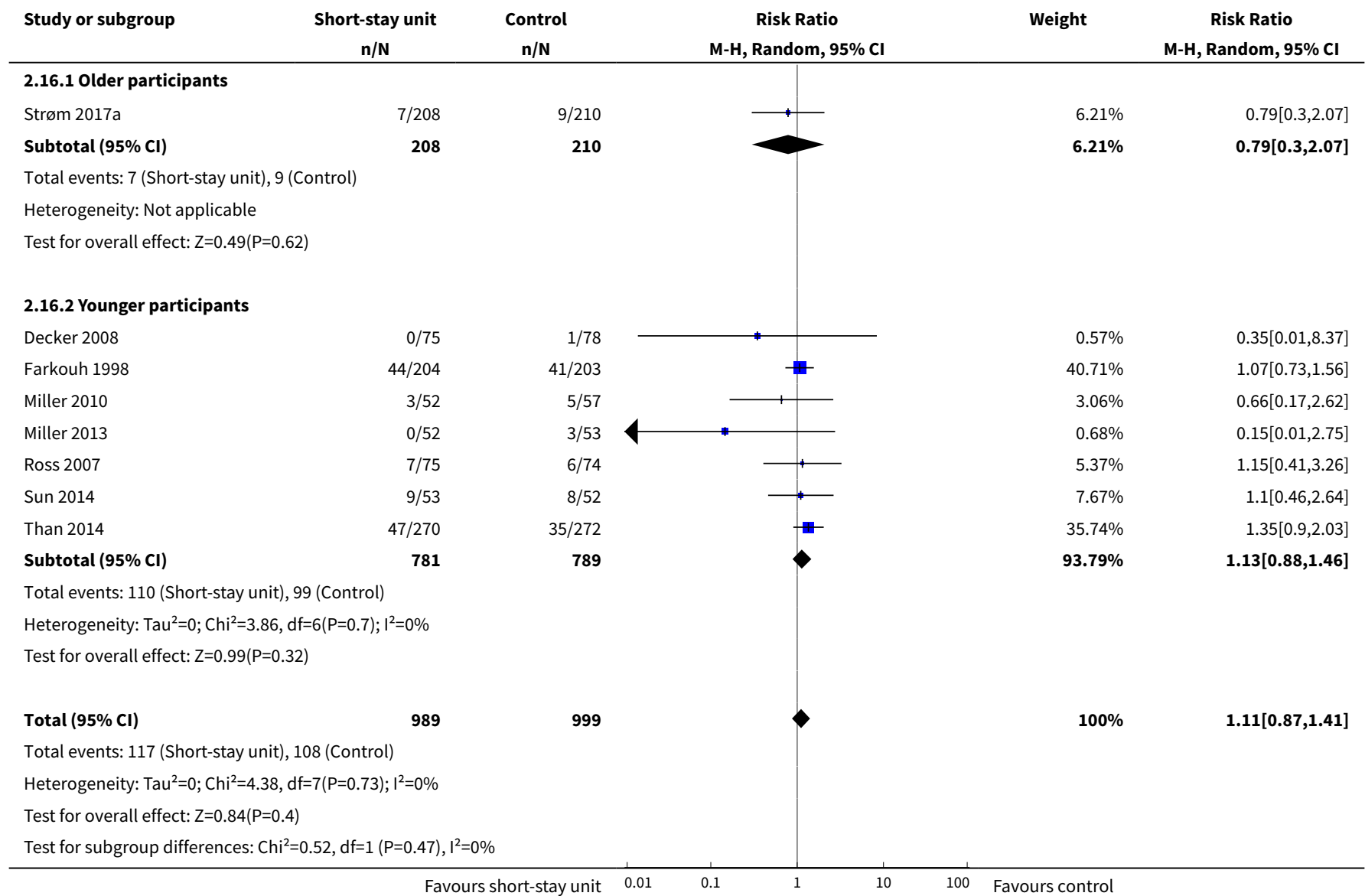

Analysis 2.17. Comparison 2 Serious adverse events in participants treated in shortstay unit vs usual care, Outcome 17 Serious adverse events at time point closest to 90 days.

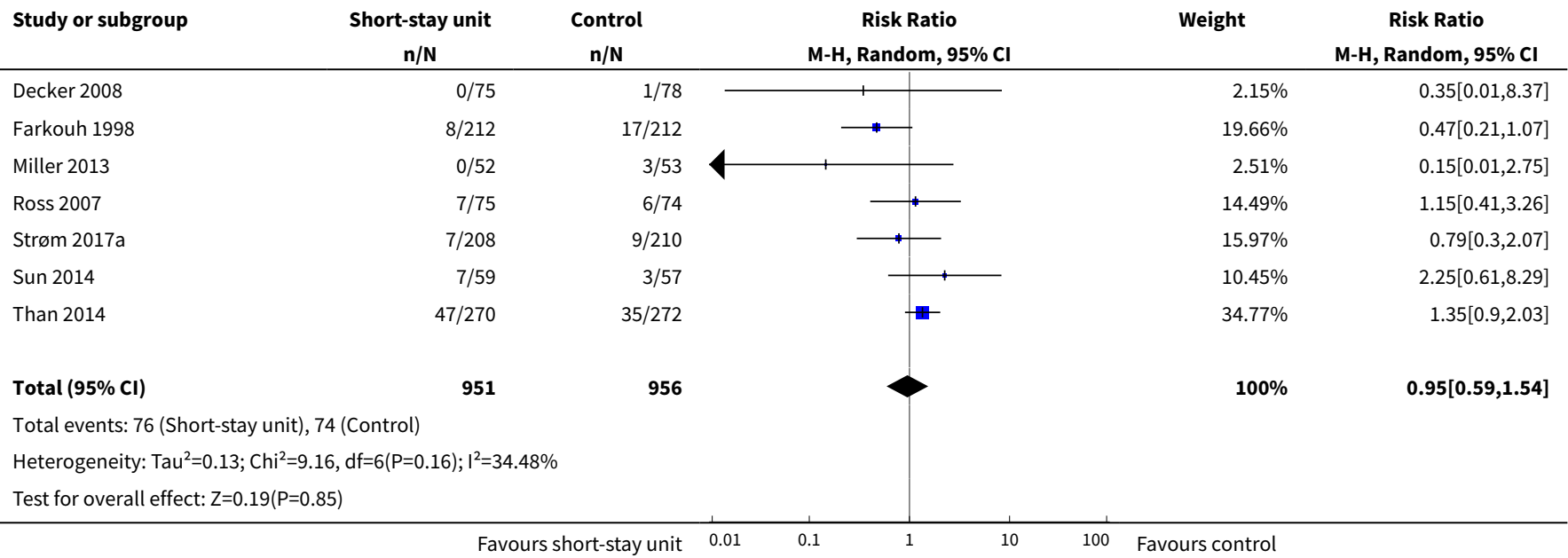


Analysis 2.18. Comparison 2 Serious adverse events in participants treated in short-stay unit vs
usual care, Outcome 18 Serious adverse events at time point closest to 90 days w/o Farkouh 1998.

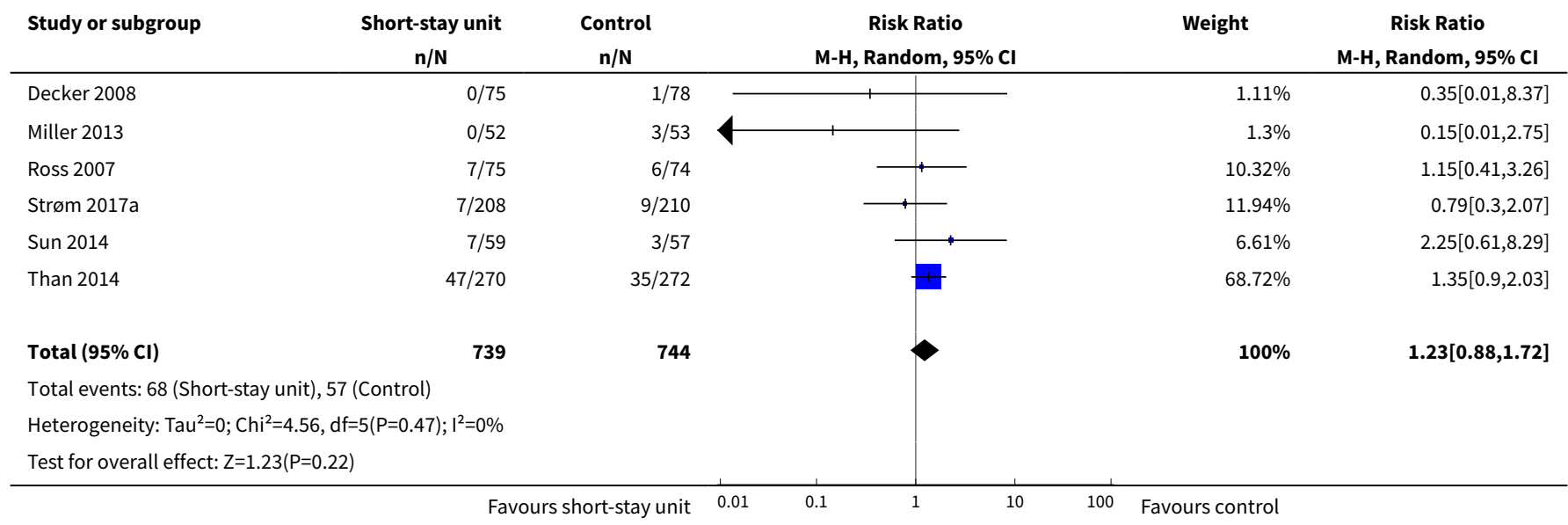

Comparison 3. Hospital readmissions in participants treated in short-stay unit vs usual care

\begin{tabular}{|c|c|c|c|c|}
\hline Outcome or subgroup title & No. of studies & $\begin{array}{l}\text { No. of partici- } \\
\text { pants }\end{array}$ & Statistical method & Effect size \\
\hline $\begin{array}{l}1 \text { Hospital readmissions at the time point } \\
\text { closest to } 90 \text { days }\end{array}$ & 8 & 1753 & $\begin{array}{l}\text { Risk Ratio (M-H, Random, } \\
95 \% \mathrm{Cl})\end{array}$ & $0.80[0.54,1.19]$ \\
\hline $\begin{array}{l}2 \text { Hospital readmissions at the time point } \\
\text { closest to } 90 \text { days best-worst case scenario }\end{array}$ & 8 & 1795 & $\begin{array}{l}\text { Risk Ratio (M-H, Random, } \\
95 \% \mathrm{Cl})\end{array}$ & $0.72[0.48,1.09]$ \\
\hline $\begin{array}{l}3 \text { Hospital readmissions at the time point } \\
\text { closest to } 90 \text { days worst-best case scenario }\end{array}$ & 8 & 1795 & $\begin{array}{l}\text { Risk Ratio (M-H, Random, } \\
95 \% \mathrm{Cl})\end{array}$ & $0.93[0.67,1.29]$ \\
\hline $\begin{array}{l}4 \text { Hospital readmissions at the time point } \\
\text { closest to } 90 \text { days published trials }\end{array}$ & 6 & 1224 & $\begin{array}{l}\text { Risk Ratio (M-H, Random, } \\
95 \% \mathrm{Cl})\end{array}$ & $0.91[0.65,1.27]$ \\
\hline $\begin{array}{l}5 \text { Hospital readmissions at the time point } \\
\text { closest to } 90 \text { days outcome assessed with- } \\
\text { in } 6 \text { months of randomisation }\end{array}$ & 8 & 1753 & $\begin{array}{l}\text { Risk Ratio (M-H, Random, } \\
95 \% \mathrm{Cl})\end{array}$ & $0.80[0.54,1.19]$ \\
\hline $\begin{array}{l}6 \text { Hospital readmissions at the time point } \\
\text { closest to } 90 \text { days multipurpose units vs } \\
\text { specialised units }\end{array}$ & 8 & 1753 & $\begin{array}{l}\text { Risk Ratio (M-H, Random, } \\
95 \% \mathrm{Cl})\end{array}$ & $0.80[0.54,1.19]$ \\
\hline 6.1 Multipurpose units & 7 & 1588 & $\begin{array}{l}\text { Risk Ratio (M-H, Random, } \\
95 \% \mathrm{Cl})\end{array}$ & $0.78[0.51,1.18]$ \\
\hline 6.2 Specialised units & 1 & 165 & $\begin{array}{l}\text { Risk Ratio (M-H, Random, } \\
95 \% \mathrm{Cl})\end{array}$ & $1.27[0.35,4.55]$ \\
\hline $\begin{array}{l}7 \text { Hospital readmissions at the time point } \\
\text { closest to } 90 \text { days non-protocol-based vs } \\
\text { protocol-based care }\end{array}$ & 8 & 1753 & $\begin{array}{l}\text { Risk Ratio (M-H, Random, } \\
95 \% \mathrm{Cl})\end{array}$ & $0.80[0.54,1.19]$ \\
\hline
\end{tabular}




\begin{tabular}{|c|c|c|c|c|}
\hline Outcome or subgroup title & No. of studies & $\begin{array}{l}\text { No. of partici- } \\
\text { pants }\end{array}$ & Statistical method & Effect size \\
\hline 7.1 Non-protocol-based care & 2 & 529 & $\begin{array}{l}\text { Risk Ratio (M-H, Random, } \\
95 \% \mathrm{Cl})\end{array}$ & $0.75[0.26,2.11]$ \\
\hline 7.2 Protocol-based care & 6 & 1224 & $\begin{array}{l}\text { Risk Ratio (M-H, Random, } \\
95 \% \mathrm{Cl})\end{array}$ & $0.91[0.65,1.27]$ \\
\hline $\begin{array}{l}8 \text { Hospital readmissions at the time point } \\
\text { closest to } 90 \text { days older vs younger partici- } \\
\text { pants }\end{array}$ & 8 & 1753 & $\begin{array}{l}\text { Risk Ratio (M-H, Random, } \\
95 \% \mathrm{Cl})\end{array}$ & $0.80[0.54,1.19]$ \\
\hline 8.1 Older participants & 2 & 529 & $\begin{array}{l}\text { Risk Ratio (M-H, Random, } \\
95 \% \mathrm{Cl} \text { ) }\end{array}$ & $0.75[0.26,2.11]$ \\
\hline 8.2 Younger participants & 6 & 1224 & $\begin{array}{l}\text { Risk Ratio (M-H, Random, } \\
95 \% \mathrm{Cl})\end{array}$ & $0.91[0.65,1.27]$ \\
\hline $\begin{array}{l}9 \text { Hospital readmissions at the time point } \\
\text { maximum follow-up }\end{array}$ & 8 & 1731 & $\begin{array}{l}\text { Risk Ratio (M-H, Random, } \\
95 \% \mathrm{Cl} \text { ) }\end{array}$ & $0.75[0.51,1.10]$ \\
\hline $\begin{array}{l}10 \text { Hospital readmissions at the time point } \\
\text { maximum follow-up best-worst case sce- } \\
\text { nario }\end{array}$ & 8 & 1795 & $\begin{array}{l}\text { Risk Ratio (M-H, Random, } \\
95 \% \mathrm{Cl})\end{array}$ & $0.65[0.45,0.94]$ \\
\hline $\begin{array}{l}11 \text { Hospital readmissions at the time point } \\
\text { maximum follow-up worst-best case sce- } \\
\text { nario }\end{array}$ & 8 & 1795 & $\begin{array}{l}\text { Risk Ratio (M-H, Random, } \\
95 \% \mathrm{Cl})\end{array}$ & $0.88[0.59,1.33]$ \\
\hline $\begin{array}{l}12 \text { Hospital readmissions at the time point } \\
\text { maximum follow-up published trials }\end{array}$ & 6 & 1223 & $\begin{array}{l}\text { Risk Ratio (M-H, Random, } \\
95 \% \mathrm{Cl} \text { ) }\end{array}$ & $0.77[0.50,1.18]$ \\
\hline $\begin{array}{l}13 \text { Hospital readmissions at the time point } \\
\text { maximum follow-up outcome assessed } \\
\text { within } 6 \text { months of randomisation }\end{array}$ & 8 & 1731 & $\begin{array}{l}\text { Risk Ratio (M-H, Random, } \\
95 \% \mathrm{Cl})\end{array}$ & $0.75[0.51,1.10]$ \\
\hline $\begin{array}{l}14 \text { Hospital readmissions at the time point } \\
\text { maximum follow-up multipurpose units vs } \\
\text { specialised units }\end{array}$ & 8 & 1731 & $\begin{array}{l}\text { Risk Ratio (M-H, Random, } \\
95 \% \mathrm{Cl})\end{array}$ & $0.75[0.51,1.10]$ \\
\hline 14.1 Multipurpose units & 7 & 1566 & $\begin{array}{l}\text { Risk Ratio (M-H, Random, } \\
95 \% \mathrm{Cl})\end{array}$ & $0.72[0.48,1.08]$ \\
\hline 14.2 Specialised units & 1 & 165 & $\begin{array}{l}\text { Risk Ratio (M-H, Random, } \\
95 \% \mathrm{Cl} \text { ) }\end{array}$ & $1.27[0.35,4.55]$ \\
\hline $\begin{array}{l}15 \text { Hospital readmissions at the time point } \\
\text { maximum follow-up non-protocol-based } \\
\text { vs protocol-based care }\end{array}$ & 8 & 1731 & $\begin{array}{l}\text { Risk Ratio (M-H, Random, } \\
95 \% \mathrm{Cl})\end{array}$ & $0.75[0.51,1.10]$ \\
\hline 15.1 Non-protocol-based care & 2 & 508 & $\begin{array}{l}\text { Risk Ratio (M-H, Random, } \\
95 \% \mathrm{Cl} \text { ) }\end{array}$ & $0.73[0.27,1.94]$ \\
\hline 15.2 Protocol-based care & 6 & 1223 & $\begin{array}{l}\text { Risk Ratio (M-H, Random, } \\
95 \% \mathrm{Cl} \text { ) }\end{array}$ & $0.77[0.50,1.18]$ \\
\hline
\end{tabular}




\begin{tabular}{|c|c|c|c|c|}
\hline Outcome or subgroup title & No. of studies & $\begin{array}{l}\text { No. of partici- } \\
\text { pants }\end{array}$ & Statistical method & Effect size \\
\hline $\begin{array}{l}16 \text { Hospital readmissions at the time point } \\
\text { maximum follow-up older vs younger par- } \\
\text { ticipants }\end{array}$ & 8 & 1731 & $\begin{array}{l}\text { Risk Ratio (M-H, Random, } \\
95 \% \mathrm{Cl})\end{array}$ & $0.75[0.51,1.10]$ \\
\hline 16.1 Older participants & 2 & 508 & $\begin{array}{l}\text { Risk Ratio (M-H, Random, } \\
95 \% \mathrm{Cl})\end{array}$ & $0.73[0.27,1.94]$ \\
\hline 16.2 Younger participants & 6 & 1223 & $\begin{array}{l}\text { Risk Ratio (M-H, Random, } \\
95 \% \mathrm{Cl} \text { ) }\end{array}$ & $0.77[0.50,1.18]$ \\
\hline $\begin{array}{l}17 \text { Hospital readmissions at the time point } \\
\text { closest to } 90 \text { days w/o Strøm } 2017 \text { a }\end{array}$ & 7 & 1351 & $\begin{array}{l}\text { Risk Ratio (M-H, Random, } \\
95 \% \mathrm{Cl} \text { ) }\end{array}$ & $0.98[0.73,1.31]$ \\
\hline $\begin{array}{l}18 \text { Hospital readmissions at the time point } \\
\text { maximum follow-up w/o Strøm } 2017 \text { a }\end{array}$ & 7 & 1329 & $\begin{array}{l}\text { Risk Ratio (M-H, Random, } \\
95 \% \mathrm{Cl})\end{array}$ & $0.85[0.59,1.22]$ \\
\hline $\begin{array}{l}19 \text { Hospital readmissions at the time point } \\
\text { maximum follow-up w/o Strøm 2017a and } \\
\text { Miller } 2010\end{array}$ & 6 & 1220 & $\begin{array}{l}\text { Risk Ratio (M-H, Random, } \\
95 \% \mathrm{Cl})\end{array}$ & $1.01[0.79,1.30]$ \\
\hline
\end{tabular}

\section{Analysis 3.1. Comparison 3 Hospital readmissions in participants treated in short-stay} unit vs usual care, Outcome 1 Hospital readmissions at the time point closest to 90 days.

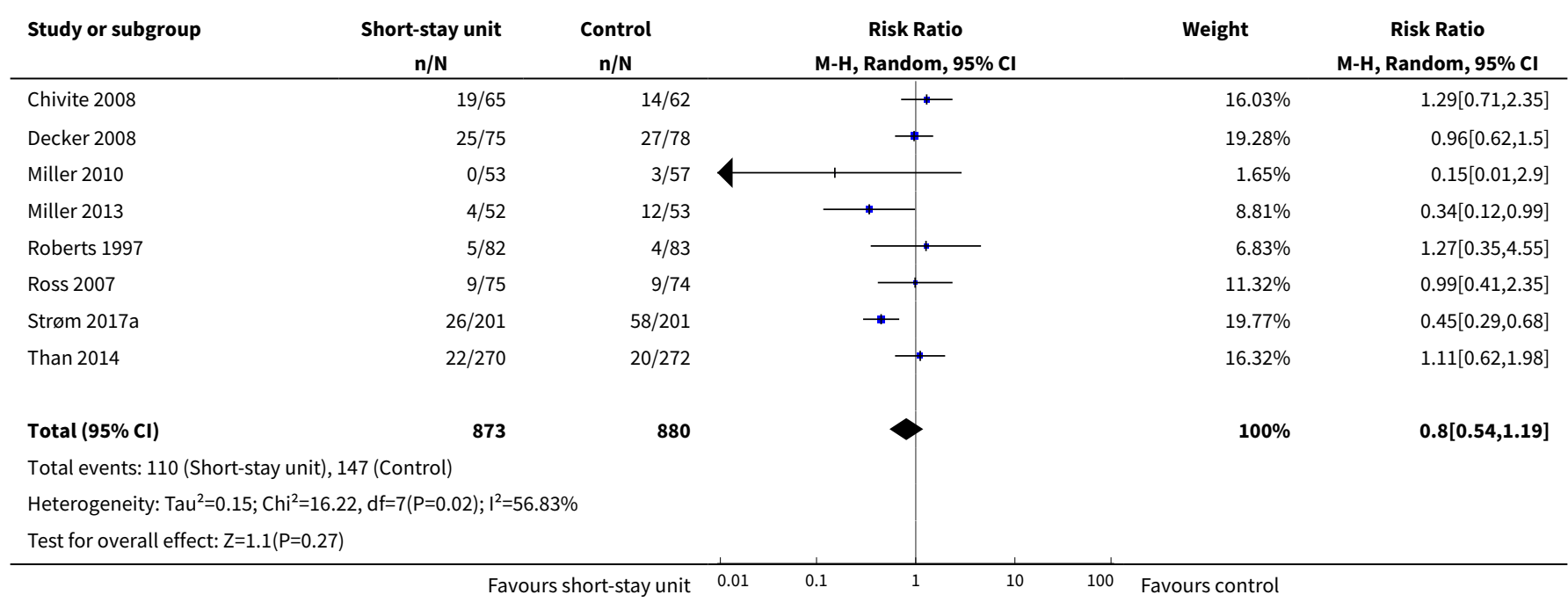

Analysis 3.2. Comparison 3 Hospital readmissions in participants treated in short-stay unit vs usual care, Outcome 2 Hospital readmissions at the time point closest to 90 days best-worst case scenario.

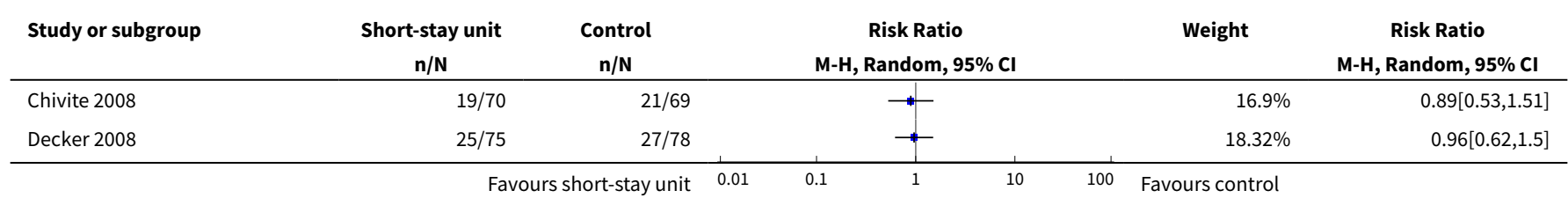




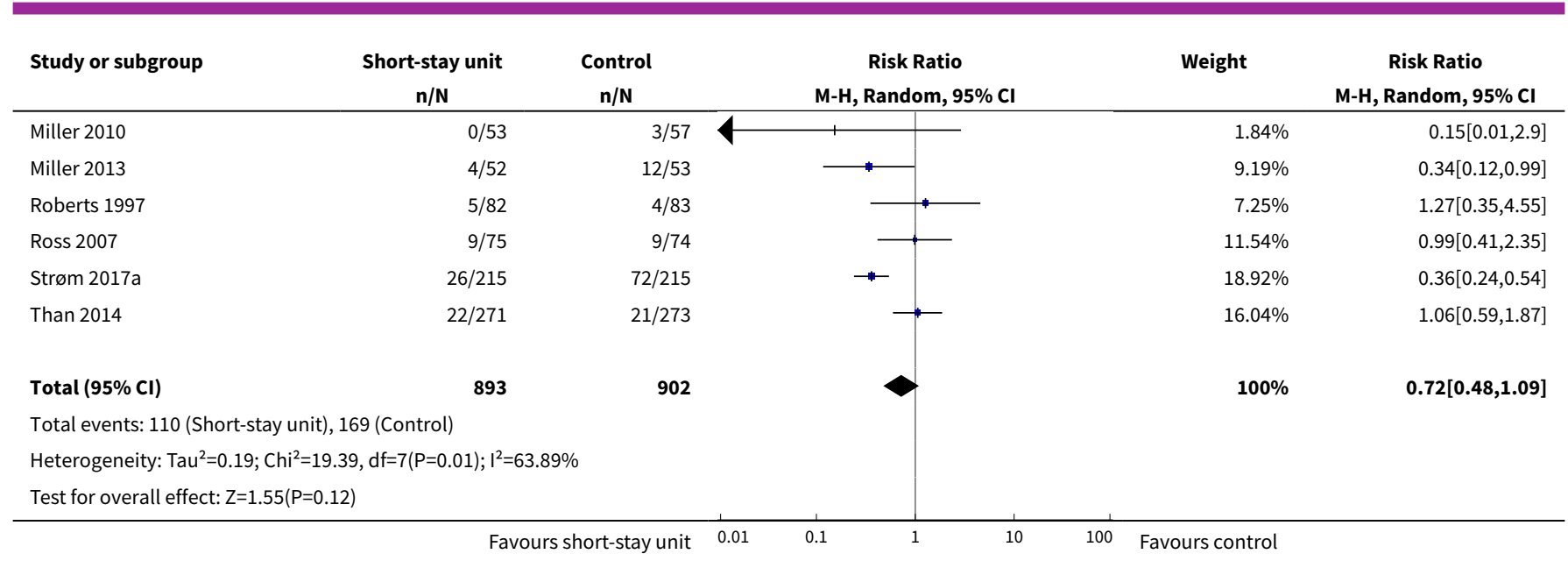

Analysis 3.3. Comparison 3 Hospital readmissions in participants treated in short-stay unit vs usual care, Outcome 3 Hospital readmissions at the time point closest to 90 days worst-best case scenario.

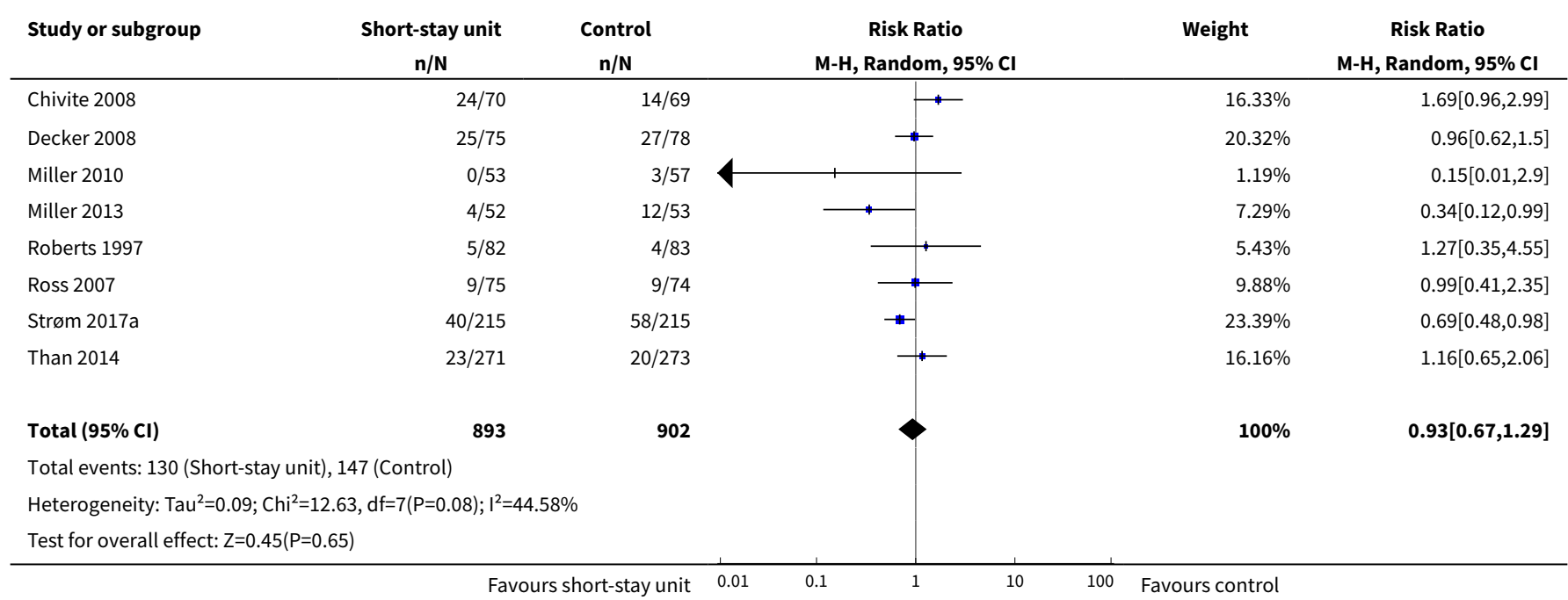

Analysis 3.4. Comparison 3 Hospital readmissions in participants treated in short-stay unit vs usual care, Outcome 4 Hospital readmissions at the time point closest to 90 days published trials.

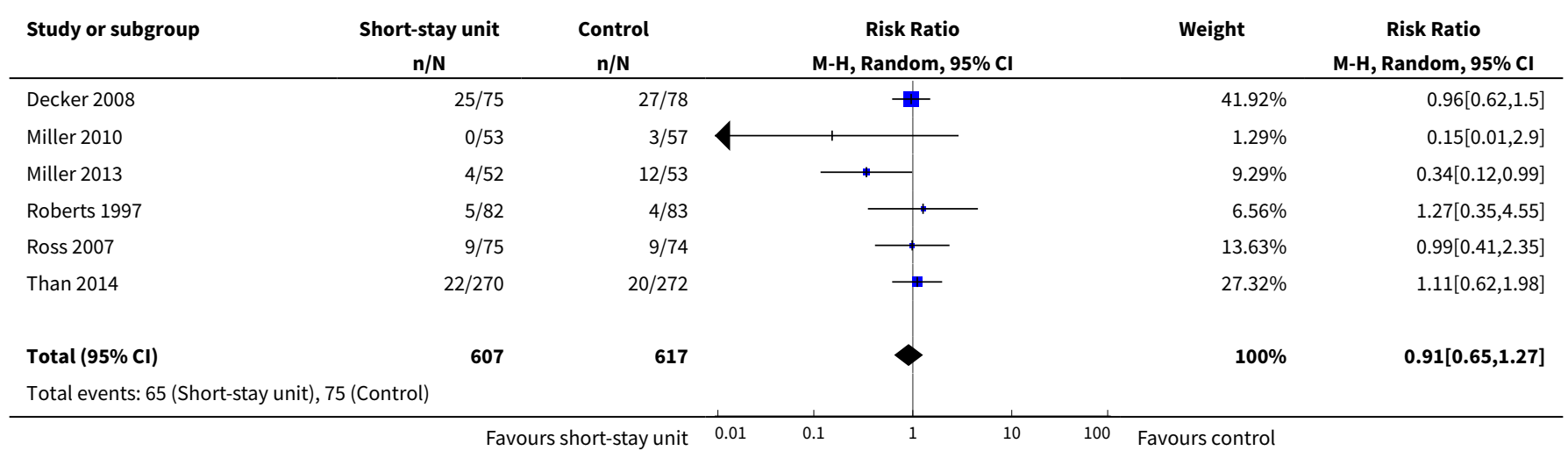




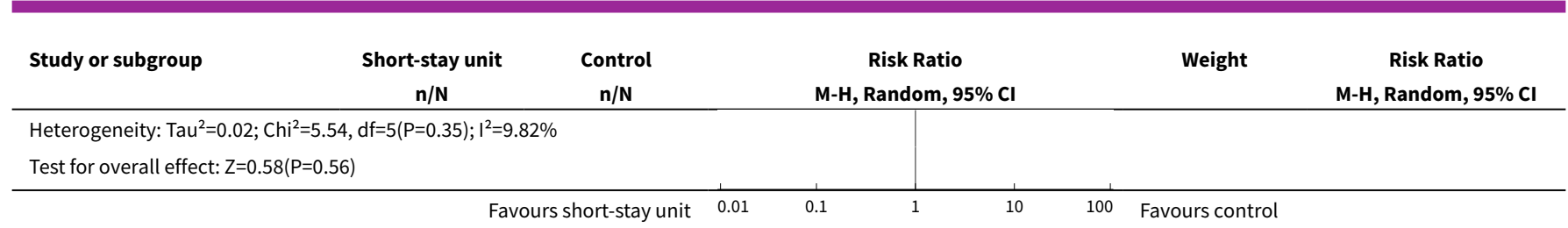

Analysis 3.5. Comparison 3 Hospital readmissions in participants treated in short-stay unit vs usual care, Outcome 5 Hospital readmissions at the time point closest to 90 days outcome assessed within 6 months of randomisation.

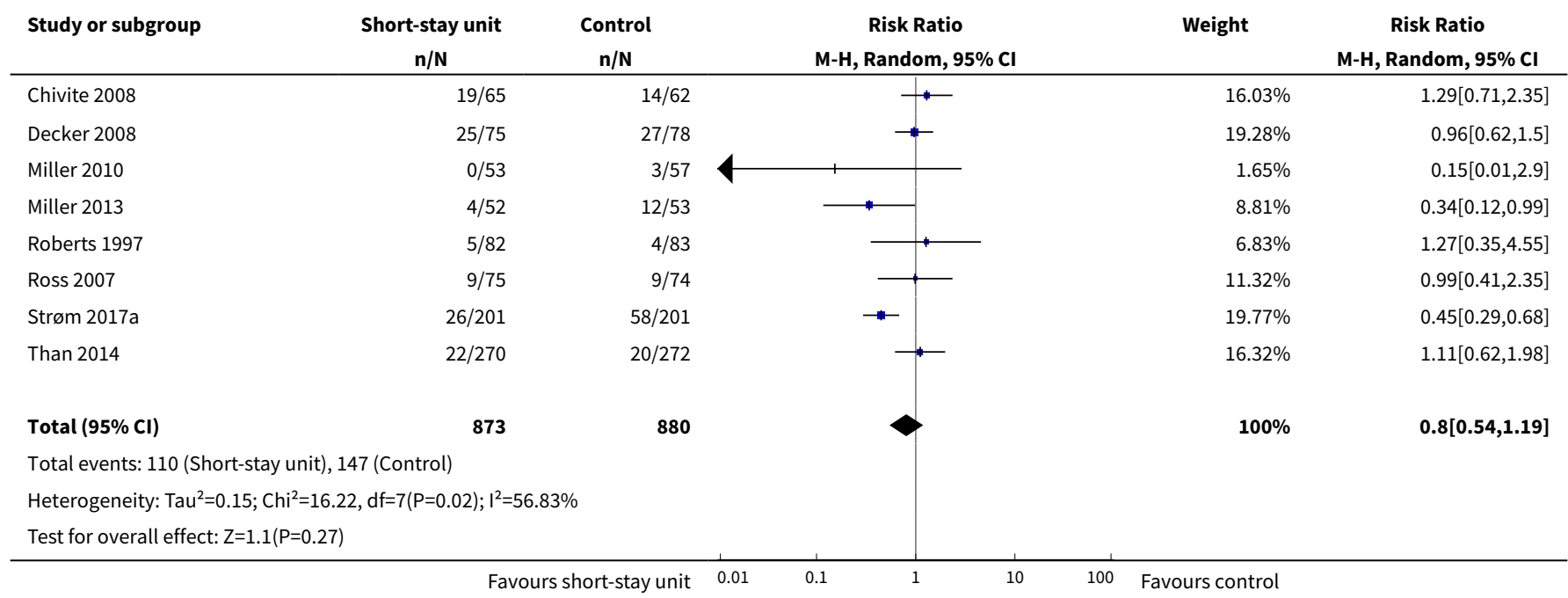

Analysis 3.6. Comparison 3 Hospital readmissions in participants treated in short-stay unit vs usual care, Outcome 6 Hospital readmissions at the time point closest to 90 days multipurpose units vs specialised units.

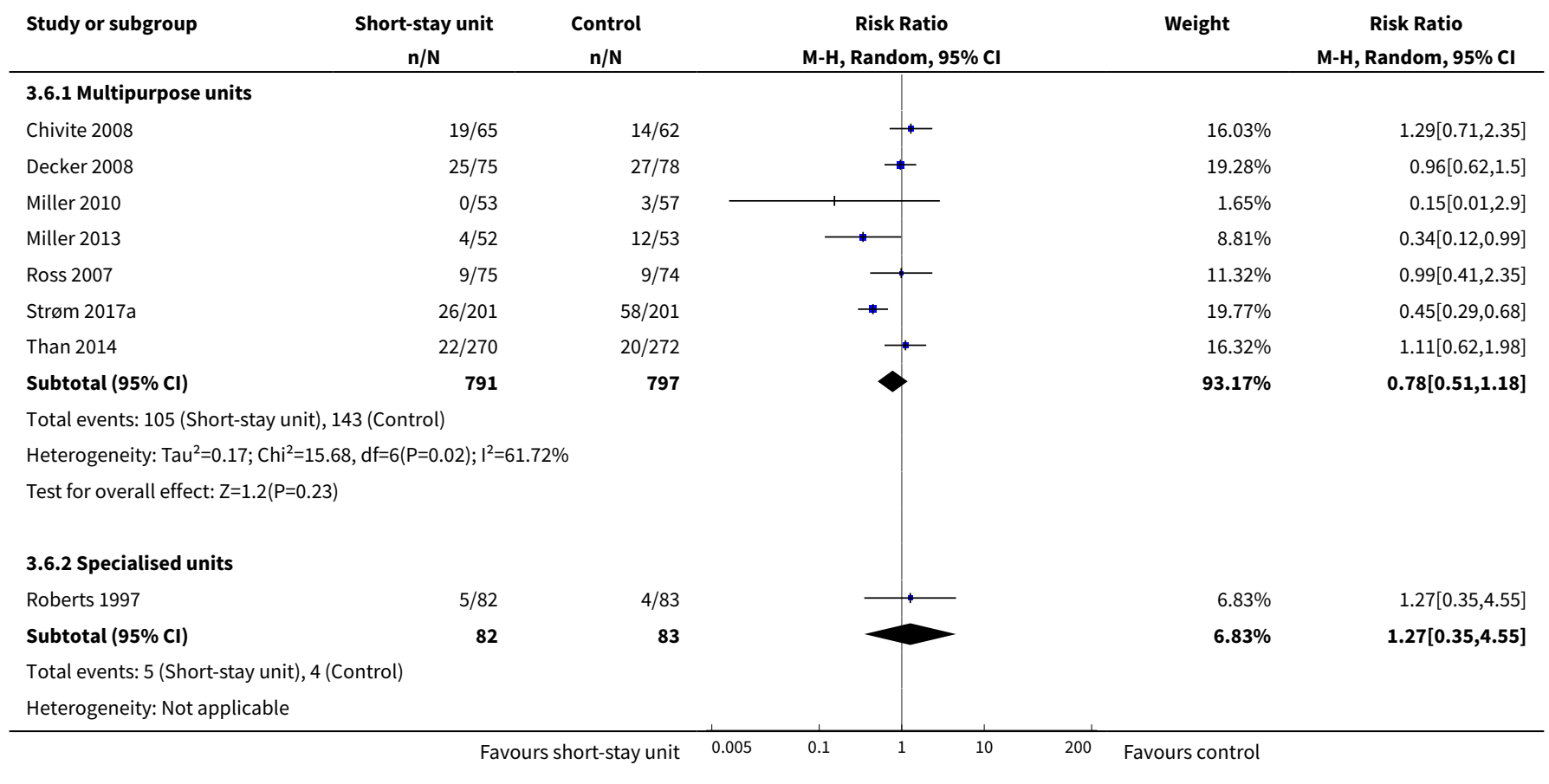




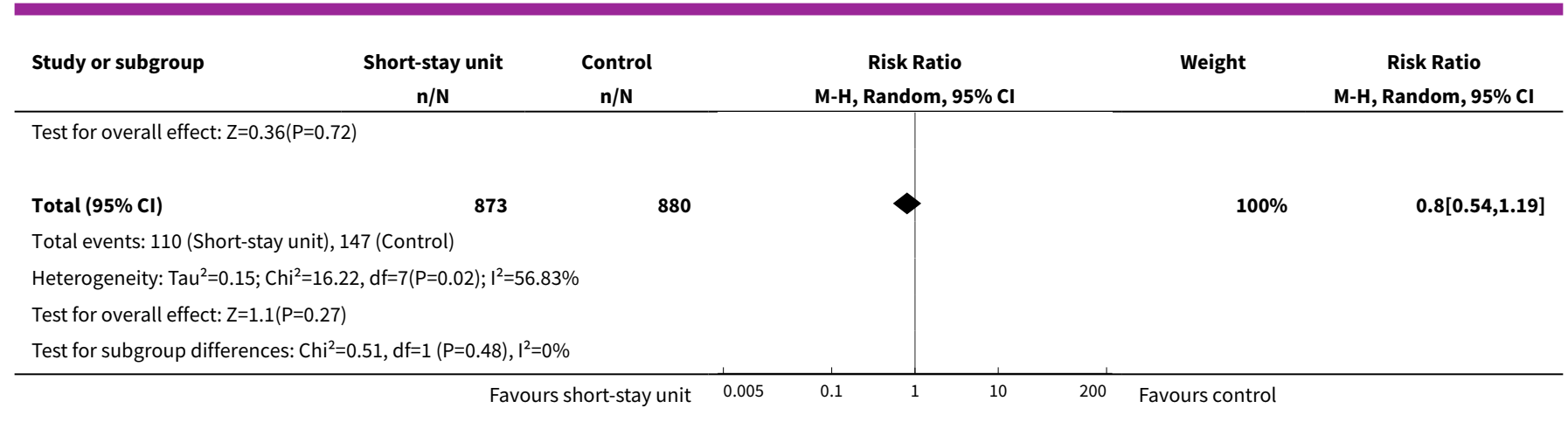

Analysis 3.7. Comparison 3 Hospital readmissions in participants treated in short-stay unit vs usual care, Outcome 7 Hospital readmissions at the time point closest to 90 days non-protocol-based vs protocol-based care.

\begin{tabular}{|c|c|c|c|c|c|}
\hline Study or subgroup & $\begin{array}{c}\text { Short-stay unit } \\
\mathrm{n} / \mathrm{N} \\
\end{array}$ & $\begin{array}{c}\text { Control } \\
\mathrm{n} / \mathrm{N}\end{array}$ & $\begin{array}{c}\text { Risk Ratio } \\
\text { M-H, Random, } 95 \% \mathrm{Cl}\end{array}$ & Weight & $\begin{array}{c}\text { Risk Ratio } \\
\text { M-H, Random, } 95 \% \mathrm{CI}\end{array}$ \\
\hline \multicolumn{6}{|c|}{ 3.7.1 Non-protocol-based care } \\
\hline Chivite 2008 & $19 / 65$ & $14 / 62$ & 7 & $16.03 \%$ & $1.29[0.71,2.35]$ \\
\hline Strøm 2017a & $26 / 201$ & $58 / 201$ & $\rightarrow$ & $19.77 \%$ & $0.45[0.29,0.68]$ \\
\hline Subtotal $(95 \% \mathrm{CI})$ & 266 & 263 & & $35.8 \%$ & $0.75[0.26,2.11]$ \\
\hline \multicolumn{6}{|c|}{ Total events: 45 (Short-stay unit), 72 (Control) } \\
\hline \multicolumn{6}{|c|}{ Heterogeneity: Tau $^{2}=0.5 ; \mathrm{Chi}^{2}=8.17, \mathrm{df}=1(\mathrm{P}=0) ; \mathrm{I}^{2}=87.76 \%$} \\
\hline \multicolumn{6}{|c|}{ Test for overall effect: $Z=0.55(P=0.58)$} \\
\hline \multicolumn{6}{|c|}{ 3.7.2 Protocol-based care } \\
\hline Decker 2008 & $25 / 75$ & $27 / 78$ & - & $19.28 \%$ & $0.96[0.62,1.5]$ \\
\hline Miller 2010 & $0 / 53$ & $3 / 57$ & & $1.65 \%$ & $0.15[0.01,2.9]$ \\
\hline Miller 2013 & $4 / 52$ & $12 / 53$ & & $8.81 \%$ & $0.34[0.12,0.99]$ \\
\hline Ross 2007 & $9 / 75$ & $9 / 74$ & - & $11.32 \%$ & $0.99[0.41,2.35]$ \\
\hline Than 2014 & $22 / 270$ & $20 / 272$ & *- & $16.32 \%$ & $1.11[0.62,1.98]$ \\
\hline Subtotal $(95 \% \mathrm{CI})$ & 607 & 617 & & $64.2 \%$ & $0.91[0.65,1.27]$ \\
\hline \multicolumn{6}{|c|}{ Total events: 65 (Short-stay unit), 75 (Control) } \\
\hline \multicolumn{6}{|c|}{ Heterogeneity: $\mathrm{Tau}^{2}=0.02 ; \mathrm{Chi}^{2}=5.54, \mathrm{df}=5(\mathrm{P}=0.35) ; \mathrm{I}^{2}=9.82 \%$} \\
\hline \multicolumn{6}{|c|}{ Test for overall effect: $Z=0.58(P=0.56)$} \\
\hline Total $(95 \% \mathrm{Cl})$ & 873 & 880 & & $100 \%$ & $0.8[0.54,1.19]$ \\
\hline \multicolumn{6}{|c|}{ Total events: 110 (Short-stay unit), 147 (Control) } \\
\hline \multicolumn{6}{|c|}{ Heterogeneity: $\mathrm{Tau}^{2}=0.15 ; \mathrm{Chi}^{2}=16.22, \mathrm{df}=7(\mathrm{P}=0.02) ; \mathrm{I}^{2}=56.83 \%$} \\
\hline \multicolumn{6}{|c|}{ Test for overall effect: $Z=1.1(P=0.27)$} \\
\hline Test for subgroup dif & $.12, \mathrm{df}=1(\mathrm{P}=0.73)$, & & & & \\
\hline
\end{tabular}

Analysis 3.8. Comparison 3 Hospital readmissions in participants treated in short-stay unit vs usual care, Outcome 8 Hospital readmissions at the time point closest to 90 days older vs younger participants.

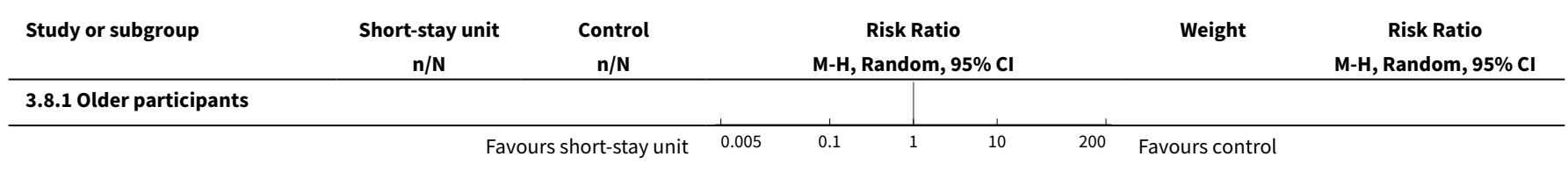




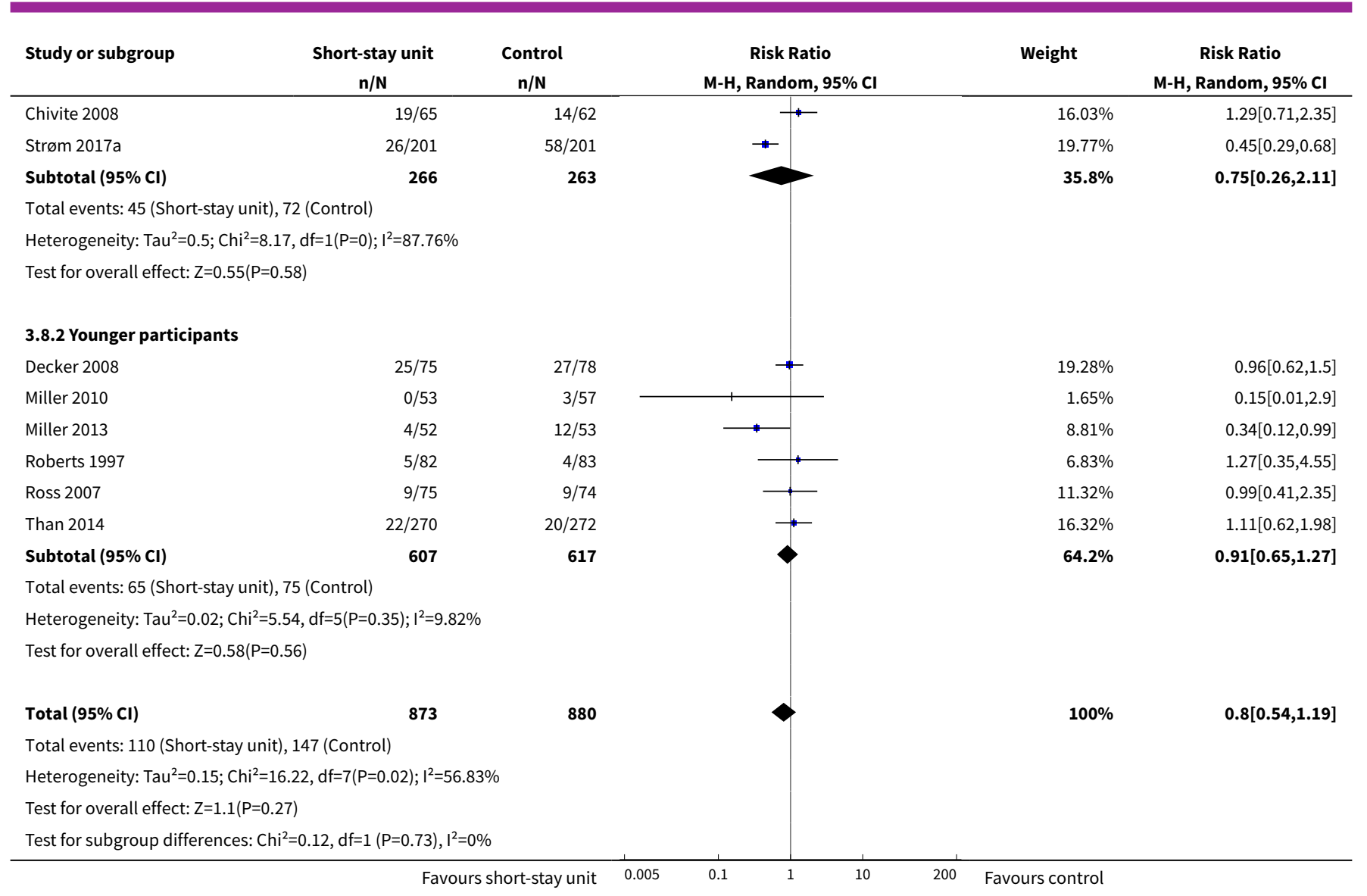

Analysis 3.9. Comparison 3 Hospital readmissions in participants treated in short-stay unit vs usual care, Outcome 9 Hospital readmissions at the time point maximum follow-up.

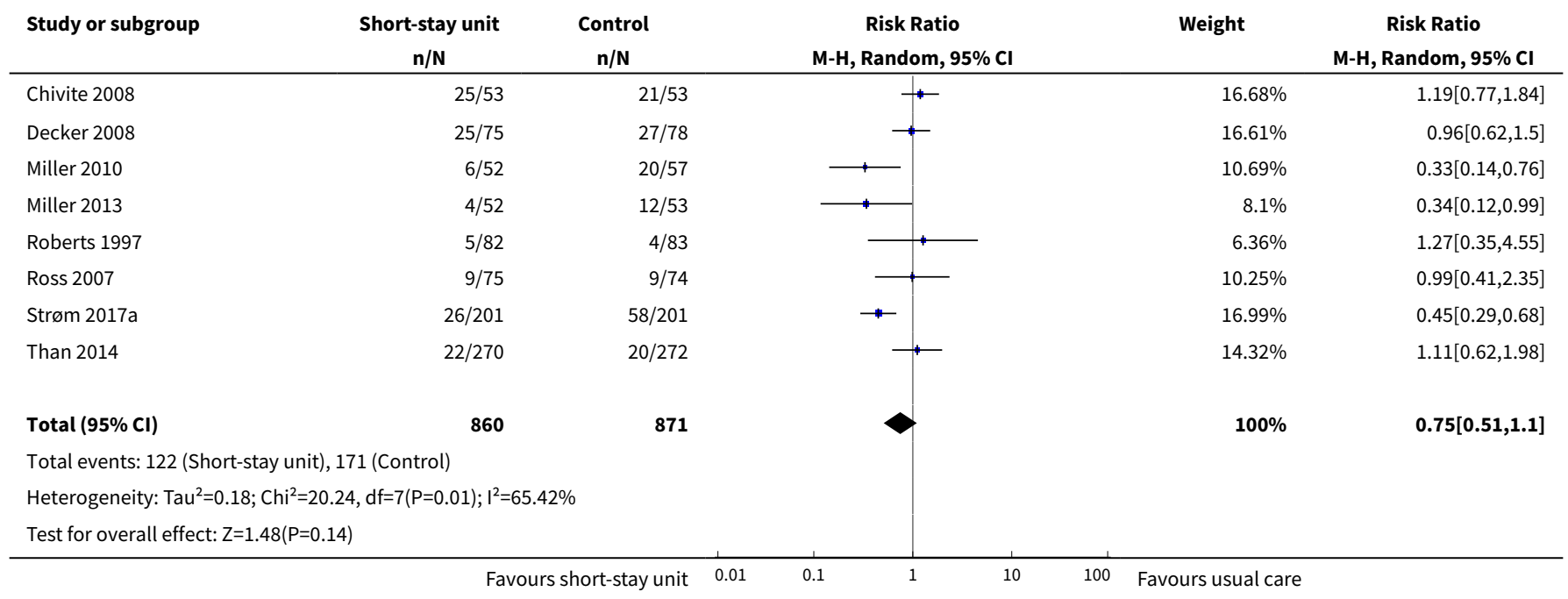


Analysis 3.10. Comparison 3 Hospital readmissions in participants treated in short-stay unit vs usual care, Outcome 10 Hospital readmissions at the time point maximum follow-up best-worst case scenario.

\begin{tabular}{|c|c|c|c|c|c|}
\hline Study or subgroup & $\begin{array}{c}\text { Short-stay unit } \\
\mathrm{n} / \mathrm{N}\end{array}$ & $\begin{array}{c}\text { Control } \\
\mathrm{n} / \mathrm{N}\end{array}$ & $\begin{array}{c}\text { Risk Ratio } \\
\text { M-H, Random, 95\% Cl }\end{array}$ & Weight & $\begin{array}{c}\text { Risk Ratio } \\
\text { M-H, Random, } 95 \% \mathrm{Cl}\end{array}$ \\
\hline Chivite 2008 & $25 / 70$ & $37 / 69$ & $\rightarrow$ & $17.62 \%$ & $0.67[0.45,0.98]$ \\
\hline Decker 2008 & $25 / 75$ & $27 / 78$ & $\rightarrow$ & $16.6 \%$ & $0.96[0.62,1.5]$ \\
\hline Miller 2010 & $6 / 53$ & $20 / 57$ & $\longrightarrow$ & $10.4 \%$ & $0.32[0.14,0.74]$ \\
\hline Miller 2013 & $4 / 52$ & $12 / 53$ & $\rightarrow$ & $7.81 \%$ & $0.34[0.12,0.99]$ \\
\hline Roberts 1997 & $5 / 82$ & $4 / 83$ & & $6.09 \%$ & $1.27[0.35,4.55]$ \\
\hline Strøm 2017a & $26 / 215$ & $72 / 215$ & $\rightarrow$ & $17.22 \%$ & $0.36[0.24,0.54]$ \\
\hline Than 2014 & $22 / 271$ & $21 / 273$ & $\longrightarrow$ & $14.3 \%$ & $1.06[0.59,1.87]$ \\
\hline Total $(95 \% \mathrm{CI})$ & 893 & 902 & & $100 \%$ & $0.65[0.45,0.94]$ \\
\hline \multicolumn{6}{|c|}{ Total events: 122 (Short-stay unit), 202 (Control) } \\
\hline \multicolumn{6}{|c|}{ Heterogeneity: $\mathrm{Tau}^{2}=0.17 ; \mathrm{Chi}^{2}=20.03, \mathrm{df}=7(\mathrm{P}=0.01) ; \mathrm{I}^{2}=65.05 \%$} \\
\hline
\end{tabular}

Analysis 3.11. Comparison 3 Hospital readmissions in participants treated in short-stay unit vs usual care, Outcome 11 Hospital readmissions at the time point maximum follow-up worst-best case scenario.

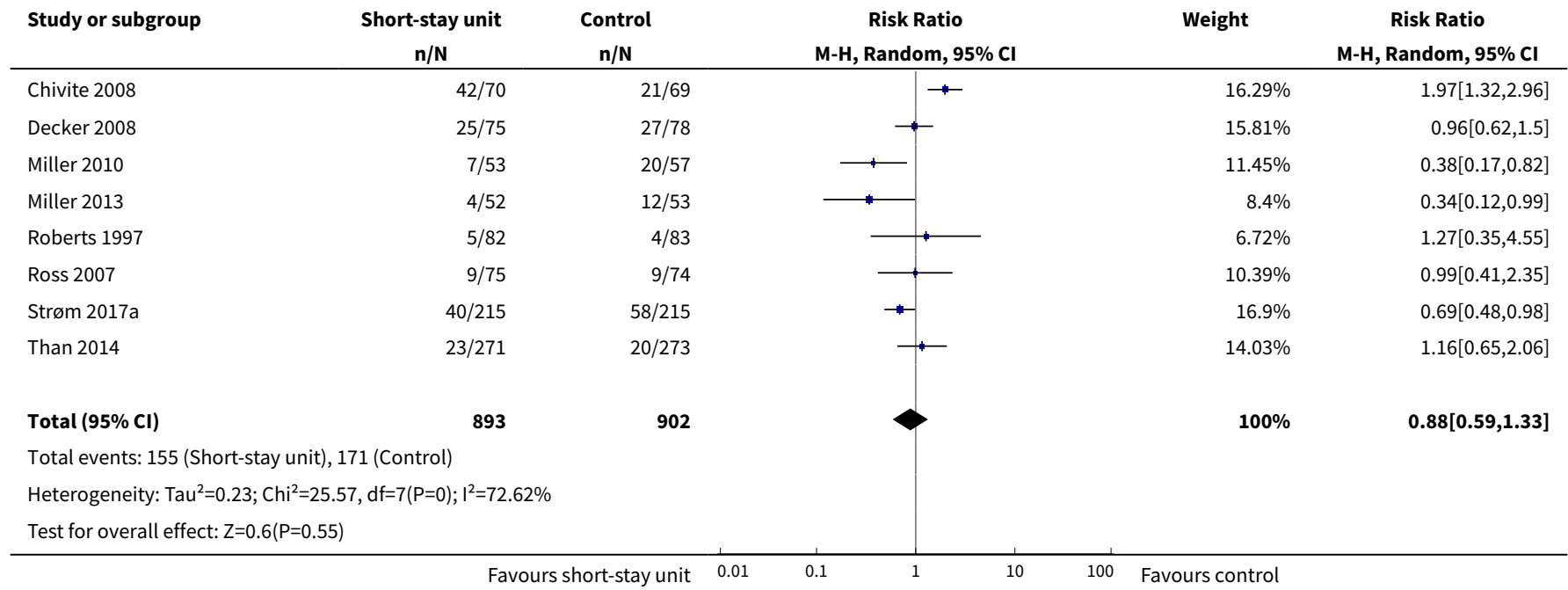

Analysis 3.12. Comparison 3 Hospital readmissions in participants treated in short-stay unit vs usual care, Outcome 12 Hospital readmissions at the time point maximum follow-up published trials.

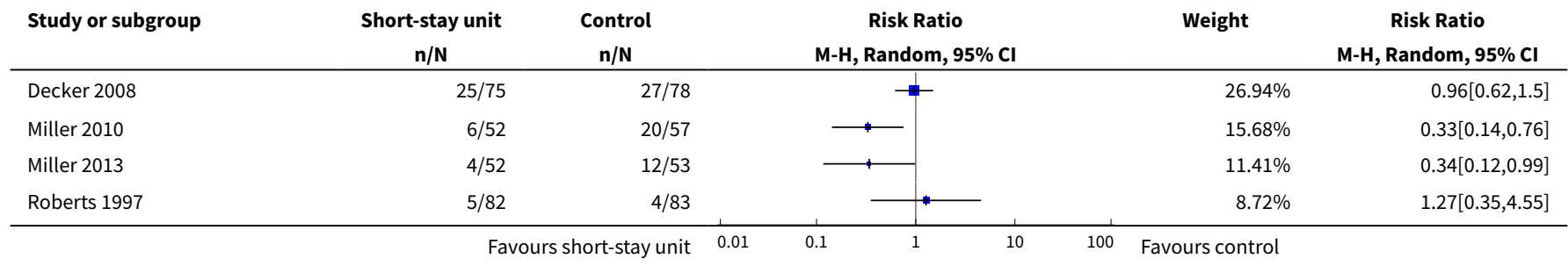




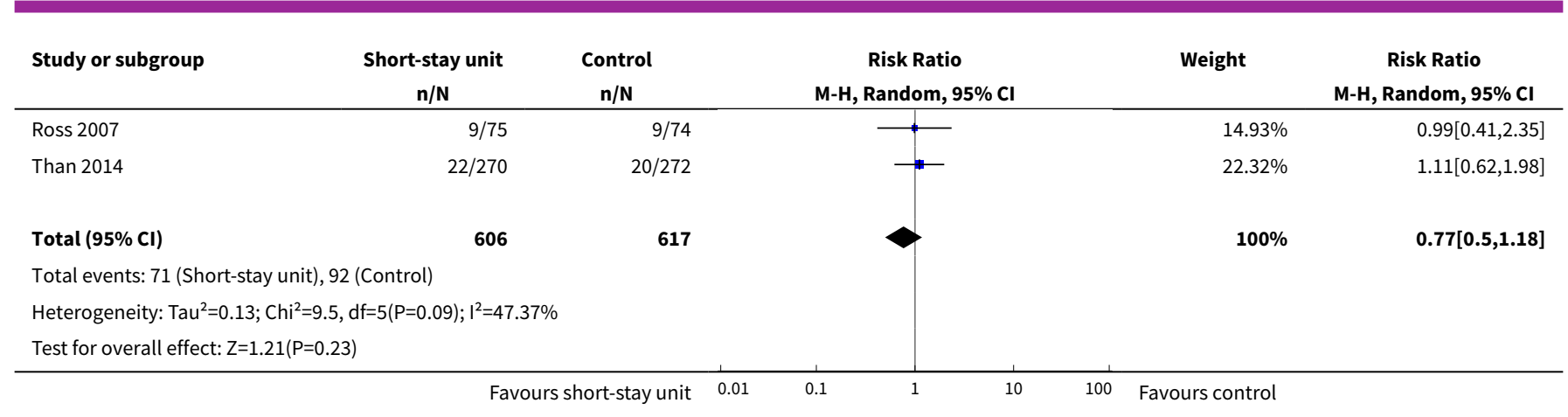

Analysis 3.13. Comparison 3 Hospital readmissions in participants treated in short-stay unit vs usual care, Outcome 13 Hospital readmissions at the time point maximum follow-up outcome assessed within 6 months of randomisation.

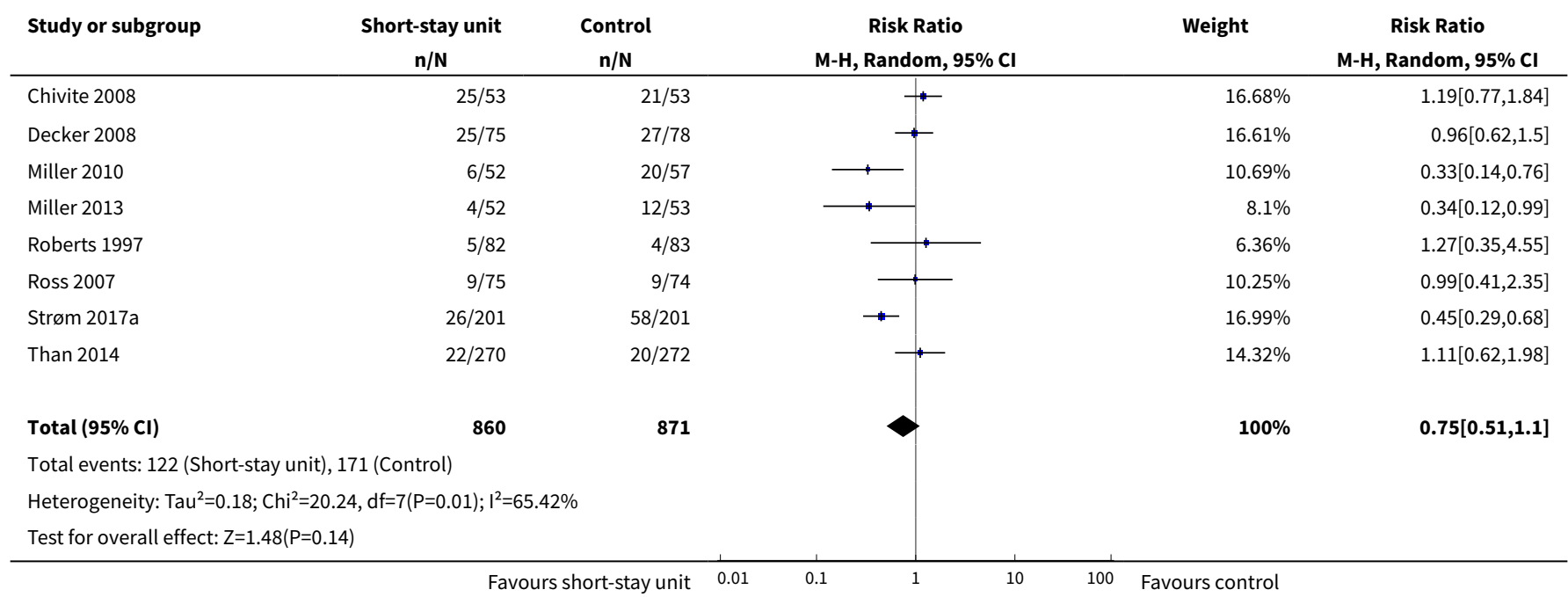

Analysis 3.14. Comparison 3 Hospital readmissions in participants treated in short-stay unit vs usual care, Outcome 14 Hospital readmissions at the time point maximum follow-up multipurpose units vs specialised units.

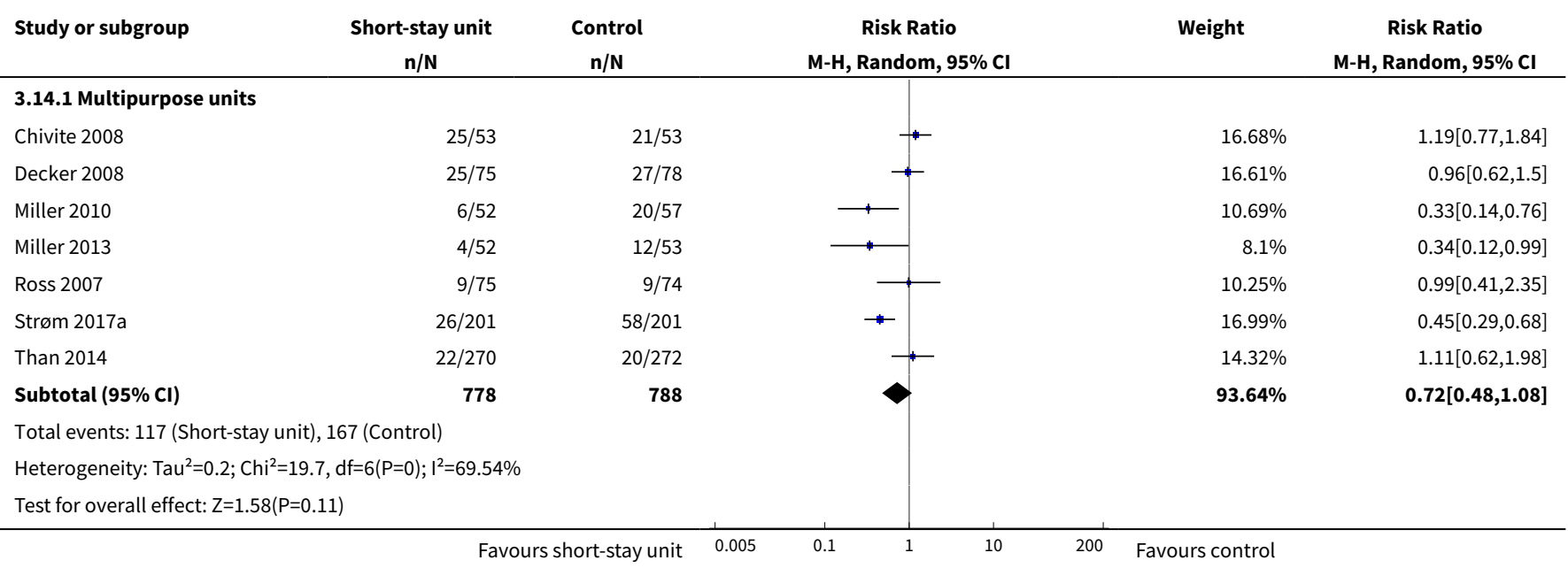




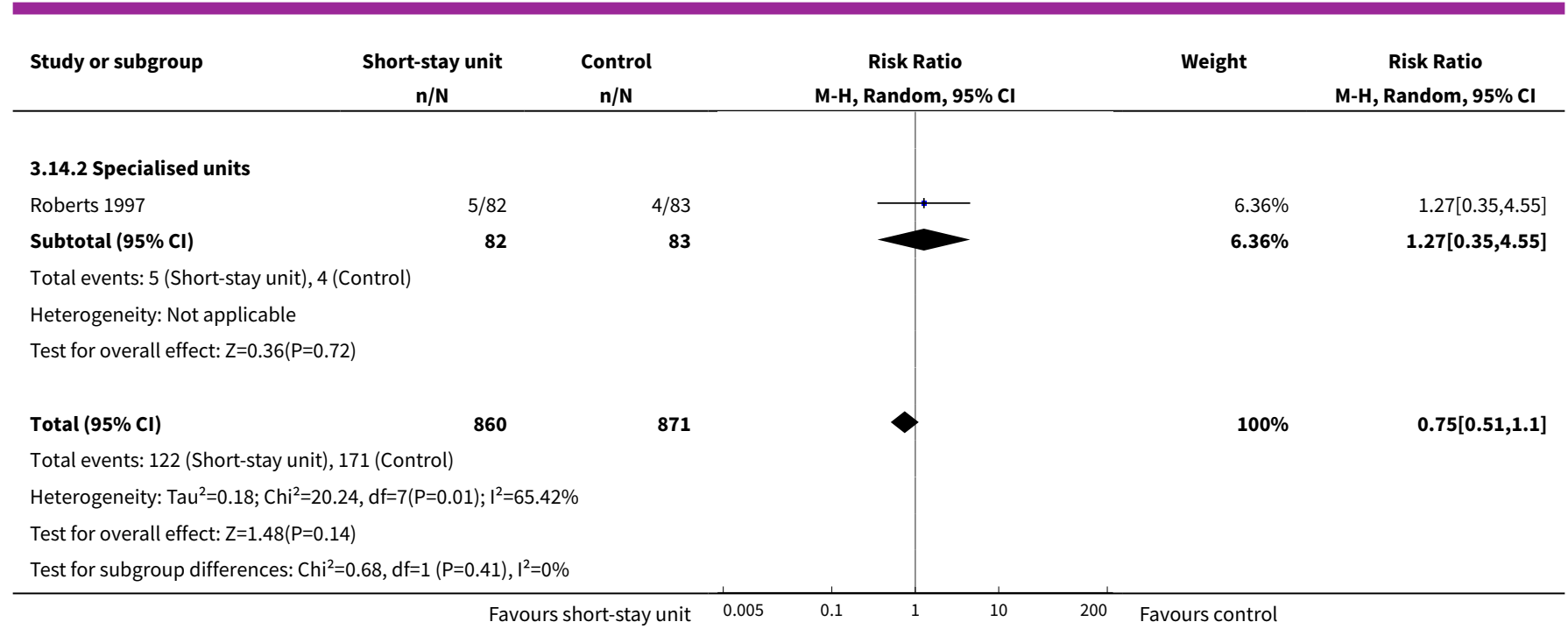

Analysis 3.15. Comparison 3 Hospital readmissions in participants treated in short-stay unit vs usual care, Outcome 15 Hospital readmissions at the time point maximum follow-up non-protocol-based vs protocol-based care.

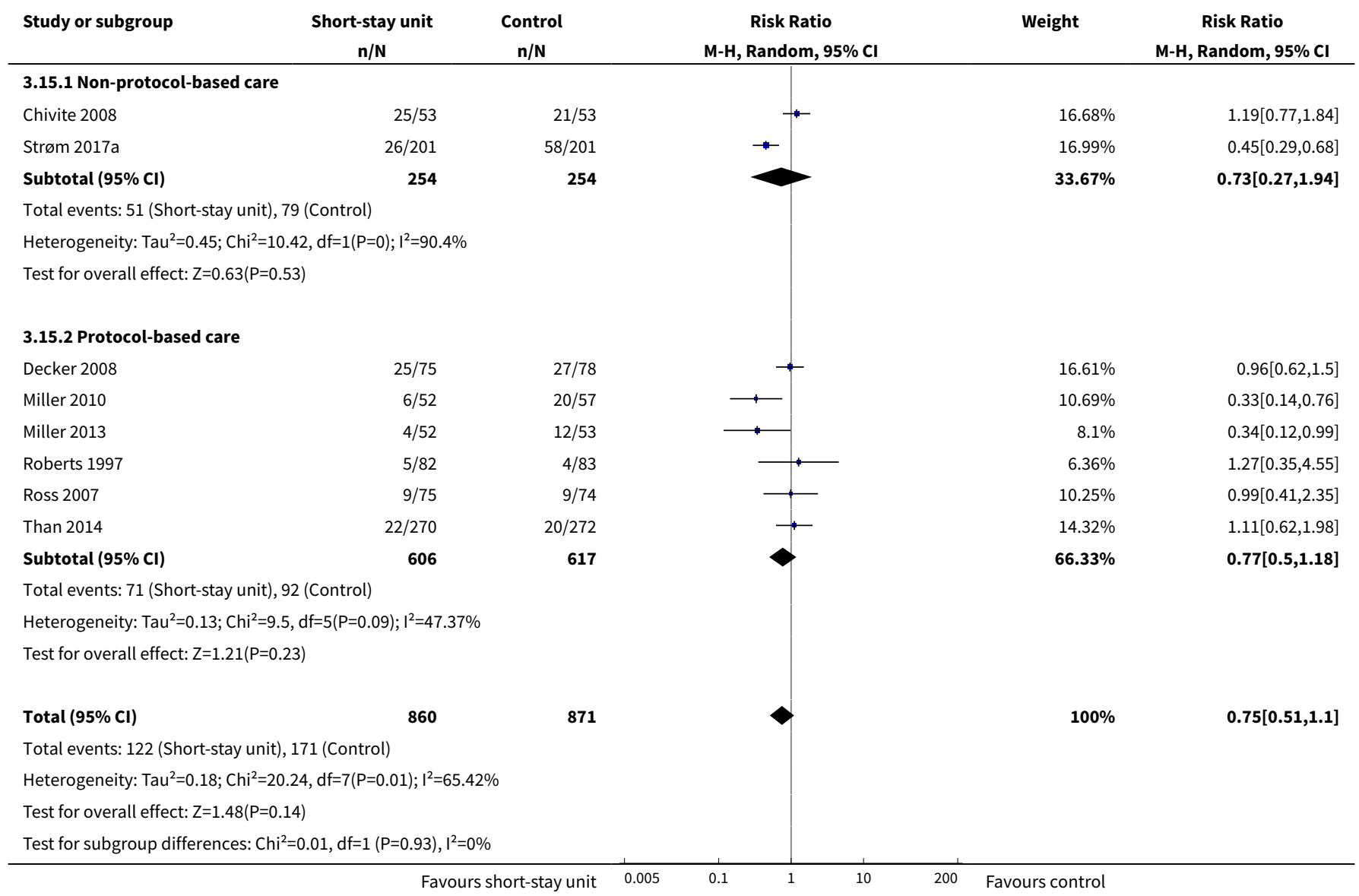


Analysis 3.16. Comparison 3 Hospital readmissions in participants treated in short-stay unit vs usual care, Outcome 16 Hospital readmissions at the time point maximum follow-up older vs younger participants.

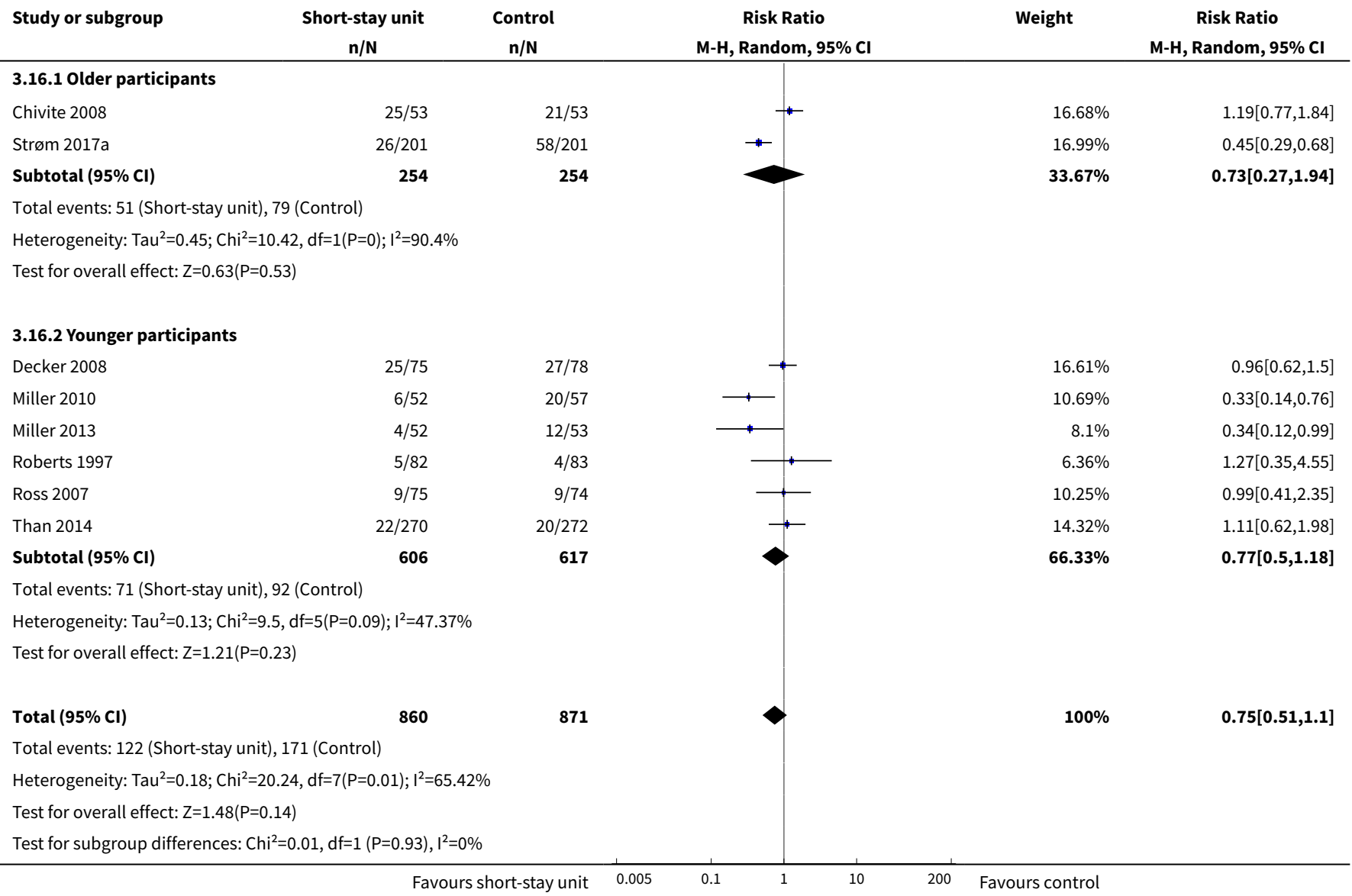

Analysis 3.17. Comparison 3 Hospital readmissions in participants treated in short-stay unit vs usual care, Outcome 17 Hospital readmissions at the time point closest to 90 days w/o Strøm 2017a.

\begin{tabular}{|c|c|c|c|c|c|}
\hline Study or subgroup & $\begin{array}{c}\text { Short-stay unit } \\
\mathrm{n} / \mathrm{N}\end{array}$ & $\begin{array}{c}\text { Control } \\
n / N\end{array}$ & $\begin{array}{c}\text { Risk Ratio } \\
\text { M-H, Random, 95\% Cl }\end{array}$ & Weight & $\begin{array}{c}\text { Risk Ratio } \\
\text { M-H, Random, } 95 \% \text { Cl }\end{array}$ \\
\hline Chivite 2008 & $19 / 65$ & $14 / 62$ & - & $20.71 \%$ & $1.29[0.71,2.35]$ \\
\hline Decker 2008 & $25 / 75$ & $27 / 78$ & $\rightarrow$ & $34.02 \%$ & $0.96[0.62,1.5]$ \\
\hline Miller 2010 & $0 / 53$ & $3 / 57$ & & $0.97 \%$ & $0.15[0.01,2.9]$ \\
\hline Miller 2013 & $4 / 52$ & $12 / 53$ & $\longrightarrow$ & $7.13 \%$ & $0.34[0.12,0.99]$ \\
\hline Roberts 1997 & $5 / 82$ & $4 / 83$ & - & $5.01 \%$ & $1.27[0.35,4.55]$ \\
\hline Than 2014 & $22 / 270$ & $20 / 272$ & - & $21.62 \%$ & $1.11[0.62,1.98]$ \\
\hline Total $(95 \% \mathrm{Cl})$ & 672 & 679 & & $100 \%$ & $0.98[0.73,1.31]$ \\
\hline \multicolumn{6}{|c|}{ Total events: 84 (Short-stay unit), 89 (Control) } \\
\hline \multicolumn{6}{|c|}{ Heterogeneity: $\mathrm{Tau}^{2}=0.01 ; \mathrm{Chi}^{2}=6.56, \mathrm{df}=6(\mathrm{P}=0.36) ; \mathrm{I}^{2}=8.58 \%$} \\
\hline Test for overall effect & & & & & \\
\hline
\end{tabular}


Analysis 3.18. Comparison 3 Hospital readmissions in participants treated in short-stay unit vs usual care, Outcome 18 Hospital readmissions at the time point maximum follow-up w/o Strøm 2017a.

\begin{tabular}{|c|c|c|c|c|c|}
\hline Study or subgroup & $\begin{array}{c}\text { Short-stay unit } \\
\mathrm{n} / \mathrm{N} \\
\end{array}$ & $\begin{array}{c}\text { Control } \\
n / N\end{array}$ & $\begin{array}{c}\text { Risk Ratio } \\
\text { M-H, Random, 95\% Cl }\end{array}$ & Weight & $\begin{array}{c}\text { Risk Ratio } \\
\text { M-H, Random, } 95 \% \text { CI }\end{array}$ \\
\hline Chivite 2008 & $25 / 53$ & $21 / 53$ & - & $22.08 \%$ & $1.19[0.77,1.84]$ \\
\hline Decker 2008 & $25 / 75$ & $27 / 78$ & $\rightarrow$ & $21.93 \%$ & $0.96[0.62,1.5]$ \\
\hline Miller 2010 & $6 / 52$ & $20 / 57$ & $\longrightarrow$ & $11.98 \%$ & $0.33[0.14,0.76]$ \\
\hline Miller 2013 & $4 / 52$ & $12 / 53$ & $\rightarrow$ & $8.52 \%$ & $0.34[0.12,0.99]$ \\
\hline Roberts 1997 & $5 / 82$ & $4 / 83$ & & $6.43 \%$ & $1.27[0.35,4.55]$ \\
\hline Than 2014 & $22 / 270$ & $20 / 272$ & * & $17.7 \%$ & $1.11[0.62,1.98]$ \\
\hline Total $(95 \% \mathrm{Cl})$ & 659 & 670 & & $100 \%$ & $0.85[0.59,1.22]$ \\
\hline \multicolumn{6}{|c|}{ Total events: 96 (Short-stay unit), 113 (Control) } \\
\hline \multicolumn{6}{|c|}{ Heterogeneity: $\mathrm{Tau}^{2}=0.1 ; \mathrm{Chi}^{2}=11.51, \mathrm{df}=6(\mathrm{P}=0.07) ; \mathrm{I}^{2}=47.88 \%$} \\
\hline \multicolumn{6}{|c|}{ Test for overall effect: $Z=0.88(P=0.38)$} \\
\hline
\end{tabular}

Analysis 3.19. Comparison 3 Hospital readmissions in participants treated in short-stay unit vs usual care, Outcome 19 Hospital readmissions at the time point maximum follow-up w/o Strøm 2017a and Miller 2010.

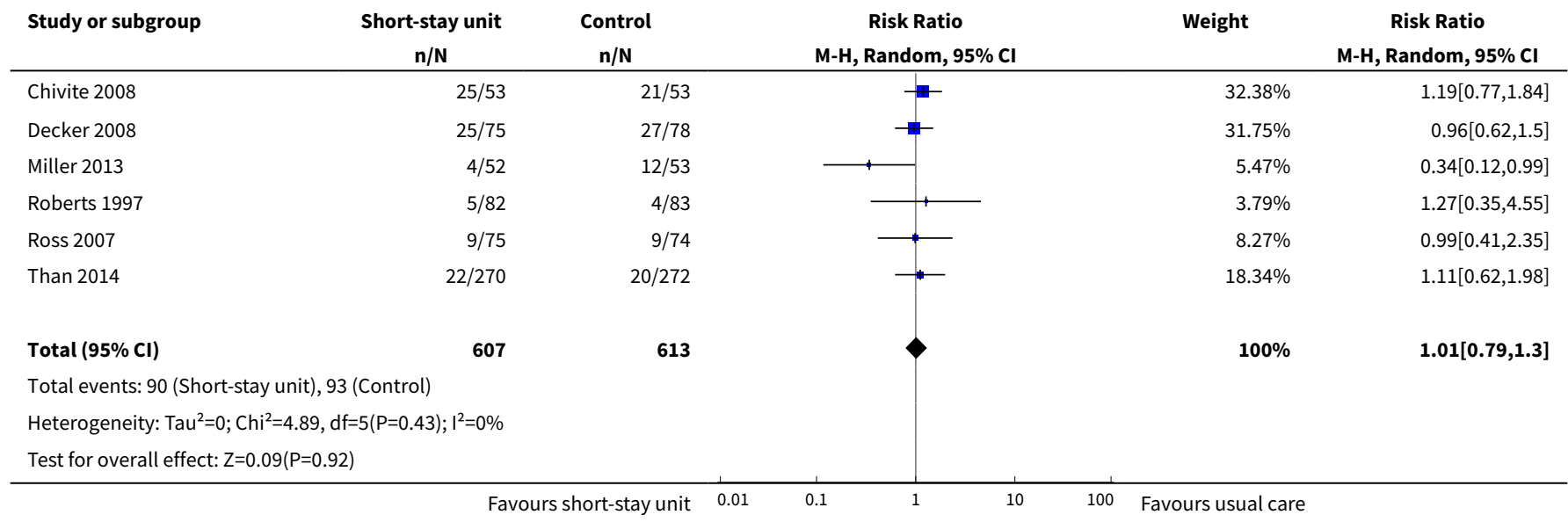

\section{ADDITIONAL TABLES}

Table 1. Funding sources

Sources of funding for included trials comparing short-stay unit hospitalisation (intervention) with usual care (control) in adult participants with internal medicine diseases and conditions

\begin{tabular}{ll}
\hline Chivite 2008 & Unclear \\
\hline Decker 2008 & Funded by a clinical research grant from the Mayo Foundation for Education and Research, USA \\
\hline Farkouh 1998 & Supported in part by grants from Aetna Health Plans (1A1575) and the Mayo Foundation, USA \\
\hline
\end{tabular}


Table 1. Funding sources (Continued)

Gomez 1996
Deseret Foundation, Intermountain Health Care, Salt Lake City, and Genetech Inc South San Fransisco California, USA
McDermott 1997 Supported by grants HHS HS07103 and HHS HS07969 from the Agency for Health Care Policy and
Research, Rockville, Md, as well as the Career Development Award from the Emergency Medicine Foundation, Dallas, Texas, USA (Dr Murphy)
Miller 2010

Funded by the Translational Science Institute of Wake Forest University School of Medicine, USA. Dr. Miller had received research support from Biosite, Schering-Plough, Siemens, and Heartscape Technologies Inc; had been a consultant for Molecular Insight and the Medicines Co; and had been a speaker for Sanofi-Aventis (indirect sponsor of a CME event). Dr. Lefebvre had received research support from Heartscape Technologies Inc. A Siemens MRI scanner was used in the trial. (Hundley). Dr. Miller had received research support from Biosite, Schering-Plough, Siemens, and Heartscape Technologies Inc; had been a consultant for Molecular Insight and the Medicines Co; and had been a speaker for Sanofi-Aventis (indirect sponsor of a CME event). A Siemens MRI scanner was used in the trial.

\begin{tabular}{ll}
\hline Roberts 1997 & Supported in part by the Agency for Health Care Policy, USA and Research grant R01-HS-07103 \\
\hline Ross 2007 & $\begin{array}{l}\text { Support from Foundation for Education and Research in Neurological Emergencies, USA and The } \\
\text { Emergency Medicine Foundation, USA }\end{array}$
\end{tabular}

Rydman 1997 Supported by grants from U.S. Department of Health \& Human Services (HHS HS07103)

Shen 2004 Mayo Foundation, USA and an investigator-initiated research grant from Medtronic Incorporated (Dr Shen). Dr Shen received support from the National Institutes of Health, USA (P50NS 32352 and R01HL 70302), and Dr Jahangir receives support from the National Institute on Aging (AG21201), the American Heart Association (0230133N), and the Mayo Foundation (CR75), all USA time of the trial, Dr. Sun was supported by NIH/ NIA grants K12 AG001004, the University of California, Los Angeles Older Americans Independence Centre P30-AG028748, and an American Geriatrics Society Dennis Jahnigen Career Development Award, all USA. Dr. Mangione was supported in part by the UCLA Robert Wood Johnson Clinical Scholars Program and the U.S. Department of Veterans Affairs (Grant \#67799), all USA. Dr. Mangione received support from the University of California, Los Angeles, Resource Centres for Minority Aging Research Centre for Health Improvement of Minority Elderly (RCMAR/CHIME) under NIH/NIA Grant P30-AG021684, and from the NIH/NCATS UCLA CTSI Grant Number UL1TR000124, all USA

\begin{tabular}{|c|c|}
\hline Strøm 2017a & $\begin{array}{l}\text { Dr Strøm received a research grant from the University of Copenhagen, Denmark and Region } \\
\text { Zealand, Denmark, and a research grant from Region Zealand Research Foundation, Denmark }\end{array}$ \\
\hline Than 2014 & $\begin{array}{l}\text { Dr Than has received funding from Alere, Abbott, Beckman, and Roche for speaking and support } \\
\text { for other research. Dr Goodacre has received funding from the UK National Institute for Health } \\
\text { Research for chest pain trials. Dr George has received funding from Abbott, Beckman Coulter, } \\
\text { and Roche for speaking. Dr Ardagh has received funding from the Health Research Council, New } \\
\text { Zealand (HRCNZ) for unrelated research. Dr Peacock has received research grants from Abbott, } \\
\text { Alere, Brahms, Novartis, Roche, and The Medicines Company; had been a consultant for Abbott, } \\
\text { Alere, Lily, The Medicines Company; had been a speaker for Bureau Abbott, Alere, and EKjmR; and } \\
\text { had ownership interest in Comprehensive Research Associates LLC, Vital Sensors, and Emergen- } \\
\text { cies in Medicine LLC. Dr Deely has received funding from the Emergency Care Foundation, NZ for } \\
\text { medical writing and HRCNZ for unrelated research, NZ. Dr Cullen has received funding from Ab- } \\
\text { bott Diagnostics, Roche, Alere, Siemens, and Radiometer Pacific for clinical trials, and from Alere, } \\
\text { Boehringer Ingelheim, Pfizer, AstraZenica, Abbott Diagnostics, and Radiometer Pacific for speaking } \\
\text { and education. Dr Richards had received speaker honoraria from Roche Dx and Alere }\end{array}$ \\
\hline
\end{tabular}


Table 2. Overview of included trials

Characteristics of included trials comparing short-stay unit hospitalisation (intervention) with usual care (control) in adult participants with internal medicine diseases and conditions

\begin{tabular}{|c|c|c|c|c|c|c|c|c|}
\hline Study ID & $\begin{array}{l}\text { Number } \\
\text { of partici- } \\
\text { pants }\end{array}$ & $\begin{array}{l}\text { Set- } \\
\text { ting/coun- } \\
\text { try }\end{array}$ & $\begin{array}{l}\text { Reason for } \\
\text { admission }\end{array}$ & $\begin{array}{l}\text { Inclusion } \\
\text { criteria }\end{array}$ & $\begin{array}{l}\text { Exclusion crite- } \\
\text { ria }\end{array}$ & $\begin{array}{l}\text { Intervention (short-stay unit } \\
\text { hospitalisation) }\end{array}$ & $\begin{array}{l}\text { Control (usual } \\
\text { care) }\end{array}$ & Outcomes \\
\hline $\begin{array}{l}\text { Chivite } 2008 \\
\text { (Trial only } \\
\text { reported in } \\
\text { abstract) }\end{array}$ & 139 & $\begin{array}{l}\text { Single cen- } \\
\text { tre/Spain }\end{array}$ & Heart failure & $\begin{array}{l}\text { Older partic- } \\
\text { ipants (age } \\
\text { limit not de- } \\
\text { fined); acute } \\
\text { decompen- } \\
\text { sated heart } \\
\text { failure; clini- } \\
\text { cal stability; } \\
\text { moderate } \\
\text { comorbid- } \\
\text { ity; moder- } \\
\text { ate disabili- } \\
\text { ty }\end{array}$ & $\begin{array}{l}\text { Secondary heart } \\
\text { failure diagno- } \\
\text { sis (defined to be } \\
\text { acute coronary } \\
\text { syndromes, se- } \\
\text { vere valve dis- } \\
\text { ease, pericardial } \\
\text { disease, isolated } \\
\text { cor pulmonale); } \\
\text { estimated sur- } \\
\text { vival < } 6 \text { months; } \\
\text { severe cognitive } \\
\text { impairment; se- } \\
\text { vere function- } \\
\text { al impairment; } \\
\text { unstable clinical } \\
\text { condition after } \\
\text { initial ED man- } \\
\text { agement (defined } \\
\text { to be hypoten- } \\
\text { sion, tachycardia, } \\
\text { electrolyte im- } \\
\text { balances, acute } \\
\text { kidney failure, or } \\
\text { need for vasoac- } \\
\text { tive drugs) }\end{array}$ & $\begin{array}{l}\text { Placement in short-stay unit, no } \\
\text { further description available in } \\
\text { abstract, but trialists informed } \\
\text { that the short-stay unit did not } \\
\text { provide specified treatment pro- } \\
\text { tocols but encouraged early mo- } \\
\text { bilisation (removal of iv lines and } \\
\text { urinary catheters, bed rest was } \\
\text { discouraged) and early discharge } \\
\text { planning (specialist physicians } \\
\text { available to implement early dis- } \\
\text { charge, cardiology consultation } \\
\text { available Monday-Friday, only } \\
\text { basic tests performed) }\end{array}$ & $\begin{array}{l}\text { Hospitalisation } \\
\text { in internal med- } \\
\text { icine services, } \\
\text { no further de- } \\
\text { scription avail- } \\
\text { able }\end{array}$ & $\begin{array}{l}\text { Length of stay } \\
\text { in hospital; } \\
\text { quality of life; } \\
\text { functional sta- } \\
\text { tus; hospital } \\
\text { readmissions; } \\
\text { mortality; exer- } \\
\text { cise capacity; } \\
\text { quality of care; } \\
\text { disease knowl- } \\
\text { edge; self-care } \\
\text { abilities; total } \\
\text { hospital costs }\end{array}$ \\
\hline Decker 2008 & 153 & $\begin{array}{l}\text { Single cen- } \\
\text { tre/USA }\end{array}$ & $\begin{array}{l}\text { Atrial fibril- } \\
\text { lation }\end{array}$ & $\begin{array}{l}\text { Age } \geq 18 \\
\text { years; atrial } \\
\text { fibrillation } \\
\text { of }<48 \mathrm{~h} \text { du- } \\
\text { ration with- } \\
\text { out haemo- } \\
\text { dynamic in- } \\
\text { stability or } \\
\text { other condi- } \\
\text { tions requir- }\end{array}$ & $\begin{array}{l}\text { Atrial fibrillation } \\
>48 \text { h duration; } \\
\text { uncertain du- } \\
\text { ration of symp- } \\
\text { toms; haemody- } \\
\text { namic instabili- } \\
\text { ty; intra-cardiac } \\
\text { thrombus; con- } \\
\text { gestive heart fail- } \\
\text { ure; class IV angi- }\end{array}$ & $\begin{array}{l}\text { Placement in ED observation unit } \\
\text { and standardised protocol: } 8 \text {-h } \\
\text { protocol including recording of } \\
\text { an ECG; chest radiograph; rou- } \\
\text { tine laboratory investigations fol- } \\
\text { lowed by pharmacologic pulse } \\
\text { rate control and continuous car- } \\
\text { diac monitoring for } 6 \mathrm{~h} \text {. Those } \\
\text { still in atrial fibrillation were se- } \\
\text { dated for electrical cardioversion }\end{array}$ & $\begin{array}{l}\text { Routine hospi- } \\
\text { tal care: ECG } \\
\text { and routine lab- } \\
\text { oratory investi- } \\
\text { gations in the } \\
\text { ED; adminis- } \\
\text { tration of an iv } \\
\text { calcium chan- } \\
\text { nel blocker or a } \\
\text { blocker for rate }\end{array}$ & $\begin{array}{l}\text { Conversion to } \\
\text { sinus rhythm or } \\
\text { rate control; re- } \\
\text { currence of atri- } \\
\text { al fibrillation; } \\
\text { adverse events; } \\
\text { utilisation of } \\
\text { healthcare re- } \\
\text { sources }\end{array}$ \\
\hline
\end{tabular}




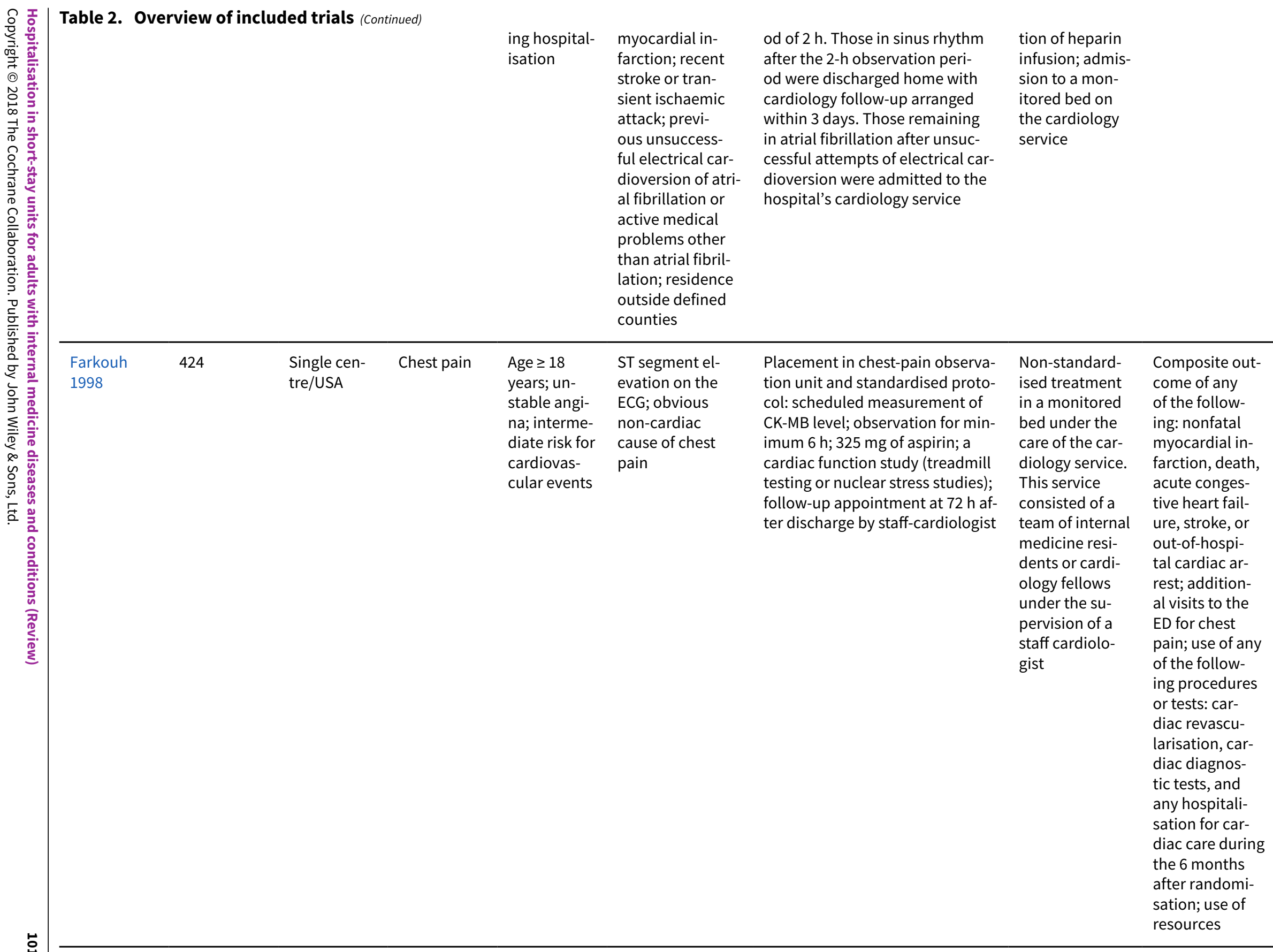




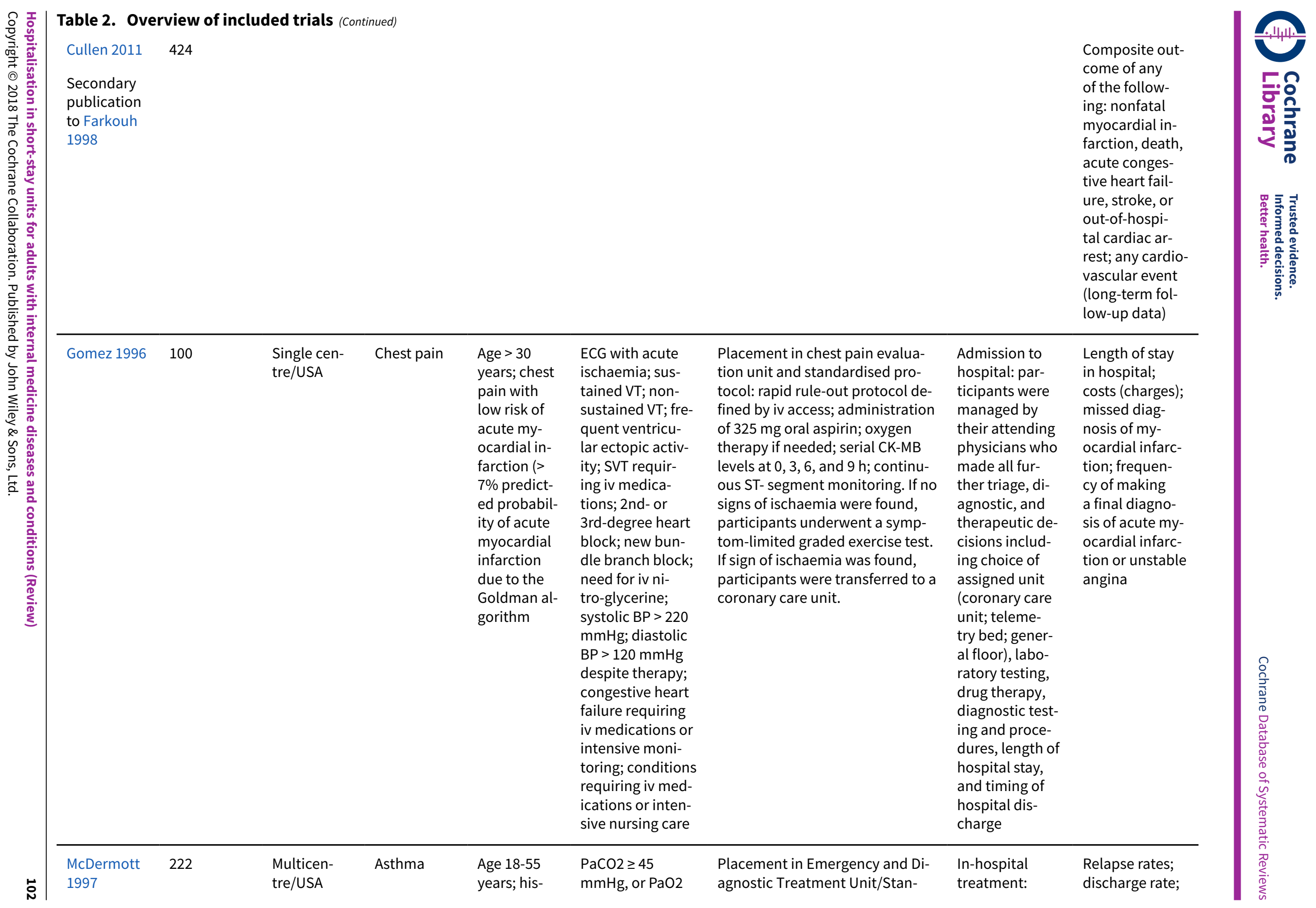




\begin{tabular}{|c|c|c|c|c|c|c|}
\hline & . & $\begin{array}{l}\text { tory of asth- } \\
\text { ma; acute } \\
\text { exacerba- } \\
\text { tion of asth- } \\
\text { ma; failure } \\
\text { to meet dis- } \\
\text { charge crite- } \\
\text { ria after } 3 \mathrm{~h} \\
\text { of ED thera- } \\
\text { py }\end{array}$ & $\begin{array}{l}\leq 55 \mathrm{mmHg} \text {, or } \\
\text { Peak Expiratory } \\
\text { Flow Rate } \leq 80 \mathrm{~L} / \\
\text { min after the first } \\
\text { adrenergic treat- } \\
\text { ment; asthma on- } \\
\text { set after age } 45 \\
\text { years and a } \geq 10 \\
\text { pack-per-year his- } \\
\text { tory of smoking; } \\
\text { a reported best } \\
\text { peak flow less } \\
\text { than a predefined } \\
\text { discharge crite- } \\
\text { rion; pregnan- } \\
\text { cy; diagnosis of } \\
\text { pneumonia, con- } \\
\text { gestive heart fail- } \\
\text { ure, or restrictive } \\
\text { lung disease pri- } \\
\text { or to eligibility as- } \\
\text { sessment }\end{array}$ & $\begin{array}{l}\text { dardised protocol: scheduled ad- } \\
\text { ministration of nebuliser at } \mathrm{h} 4, \\
6,8,10 \text {, and } 12 ; \text { repeated steroid } \\
\text { dose at hour } 6 \text {. If a set of prede- } \\
\text { fined discharge criteria were met, } \\
\text { participants were discharged. } \\
\text { Discharge criteria were assessed } \\
\text { repeatedly. If discharge criteria } \\
\text { were not met within } 12 \mathrm{~h} \text {, partici- } \\
\text { pants were admitted to in-hospi- } \\
\text { tal service }\end{array}$ & $\begin{array}{l}\text { treatment in a } \\
\text { hospital ward } \\
\text { according to } \\
\text { National Asth- } \\
\text { ma Guidelines: } \\
\text { handheld neb- } \\
\text { uliser every } 2 \\
\mathrm{~h} \text { for } 3 \text { treat- } \\
\text { ments after ad- } \\
\text { mission and } \\
4 \text { times there- } \\
\text { after; } 60 \text { mg of } \\
\text { methylpred- } \\
\text { nisolone on ar- } \\
\text { rival and every } \\
6 \mathrm{~h} \text { thereafter. } \\
\text { Discharge cri- } \\
\text { teria were simi- } \\
\text { lar to the inter- } \\
\text { vention-group, } \\
\text { but participants } \\
\text { were only as- } \\
\text { sessed at time } \\
\text { of arrival at } \\
\text { ward and then } \\
\text { on daily rounds }\end{array}$ & $\begin{array}{l}\text { length of stay } \\
\text { in hospital; } \\
\text { minor mor- } \\
\text { bidity (cough, } \\
\text { wheezing, dysp- } \\
\text { noea, nocturnal } \\
\text { awakenings); } \\
\text { moderate mor- } \\
\text { bidity (major } \\
\text { lifestyle-limit- } \\
\text { ing events; e.g. } \\
\text { days missed } \\
\text { from work or } \\
\text { school, days } \\
\text { incapacitated } \\
\text { during waking } \\
\text { hours); major } \\
\text { morbidity (un- } \\
\text { scheduled visits } \\
\text { for treatment of } \\
\text { acute asthma); } \\
\text { direct medical } \\
\text { costs; patient } \\
\text { satisfaction; } \\
\text { quality of life }\end{array}$ \\
\hline $\begin{array}{l}\text { Rydman } \\
1998 \\
\text { Secondary } \\
\text { publication } \\
\text { to McDer- } \\
\text { mott } 1997\end{array}$ & $113 / 222$ & & & & & $\begin{array}{l}\text { Quality of life; } \\
\text { clinical status } \\
\text { as measured by } \\
\text { peak flow rates; } \\
\text { total costs; re- } \\
\text { lapse-free sur- } \\
\text { vival } 8 \text { weeks } \\
\text { after treatment }\end{array}$ \\
\hline $\begin{array}{l}\text { Rydman } \\
1999 \\
\text { Secondary } \\
\text { publication } \\
\text { to McDer- } \\
\text { mott } 1997\end{array}$ & $163 / 222$ & & & & & $\begin{array}{l}\text { Patient satis- } \\
\text { faction; prob- } \\
\text { lems with care } \\
\text { processes }\end{array}$ \\
\hline
\end{tabular}


Single cen- Chest pain tre/USA

(

Age $\geq 18$ years; symptoms of

possible

acute coro-

nary syn-

drome; care

provider im-

pression

that inpa-

tient evalua-

tion was re-

quired; abil-

ity to be dis-

charged if

cardiac dis-

ease was

excluded;

intermedi-

ate or high

probabili-

ty of having

acute coro-

nary syn-

drome
Initial increased troponin; new ST segment elevation $(\geq 1 \mathrm{mV}$ ) or depression $(\geq 2$ $\mathrm{mV})$; inability to lie flat; systolic $\mathrm{BP}<90 \mathrm{mmHg}$; contraindications to MRI; refusal of follow-up procedures; terminal diagnosis with < 3 months to live pregnancy; renal insufficien-

cy; chronic liver disease; history of heart, liver, or kidney transplant
Placement in ED observation unit (trialists informed it was a multipurpose unit) and standardised protocol: cardiac biomarkers at 4 and $8 \mathrm{~h}$; stress cardiac MRI examination available weekdays $8 \mathrm{am}-5 \mathrm{pm}$. If the 4-h troponin I level was negative; participants could receive the stress cardiac MRI examination at the first available period. The short-stay unit was staffed by nurse practitioners or physician assistants and supervised by a board-certified emergency physician
Consultation in the ED by the admitting service as per customary prac-

tice (cardiology, internal medicine, or family medicine services); care delivery determined by the care providers and not dic tated by a trial protocol
Costs; correct admission deing to acute coronary synsis within 30 days); number of participants randomised to short-stay unit that were able to complete cardiac MRI; utilisation of health care procedures; adverse events during MRI; adverse events leading to early termination of MRI cision (accorddrome diagno-

follow-up)
3 months; preg-

could be
Placement in ED observation unit (trialists informed it was a multiprotocol: cardiac biomarkers at 4 and $8 \mathrm{~h}$; stress cardiac MRI examination available weekdays $8 \mathrm{am}-5 \mathrm{pm}$. If the $4-\mathrm{h}$ troponin I level was negative; participants could receive the stress cardiac MRI examination at the first available period. The short-stay unit was staffed by nurse practitionpurpose unit) and standardised
Usual care: consultation in the ED by the admitting service as per customary practice

(Cardiology, Internal Medicine, or Fami-

ly Medicine services); care delivery deter- 


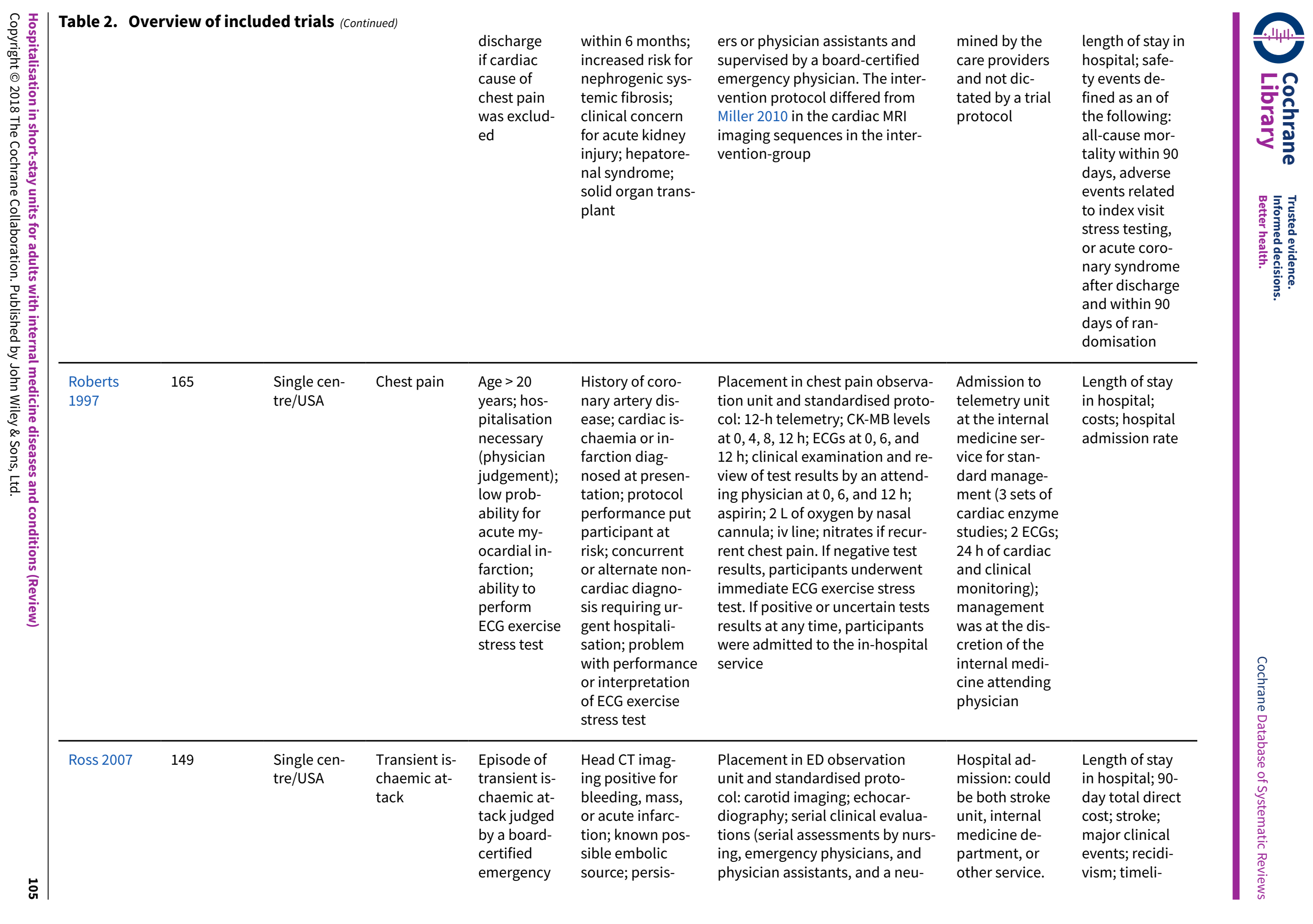




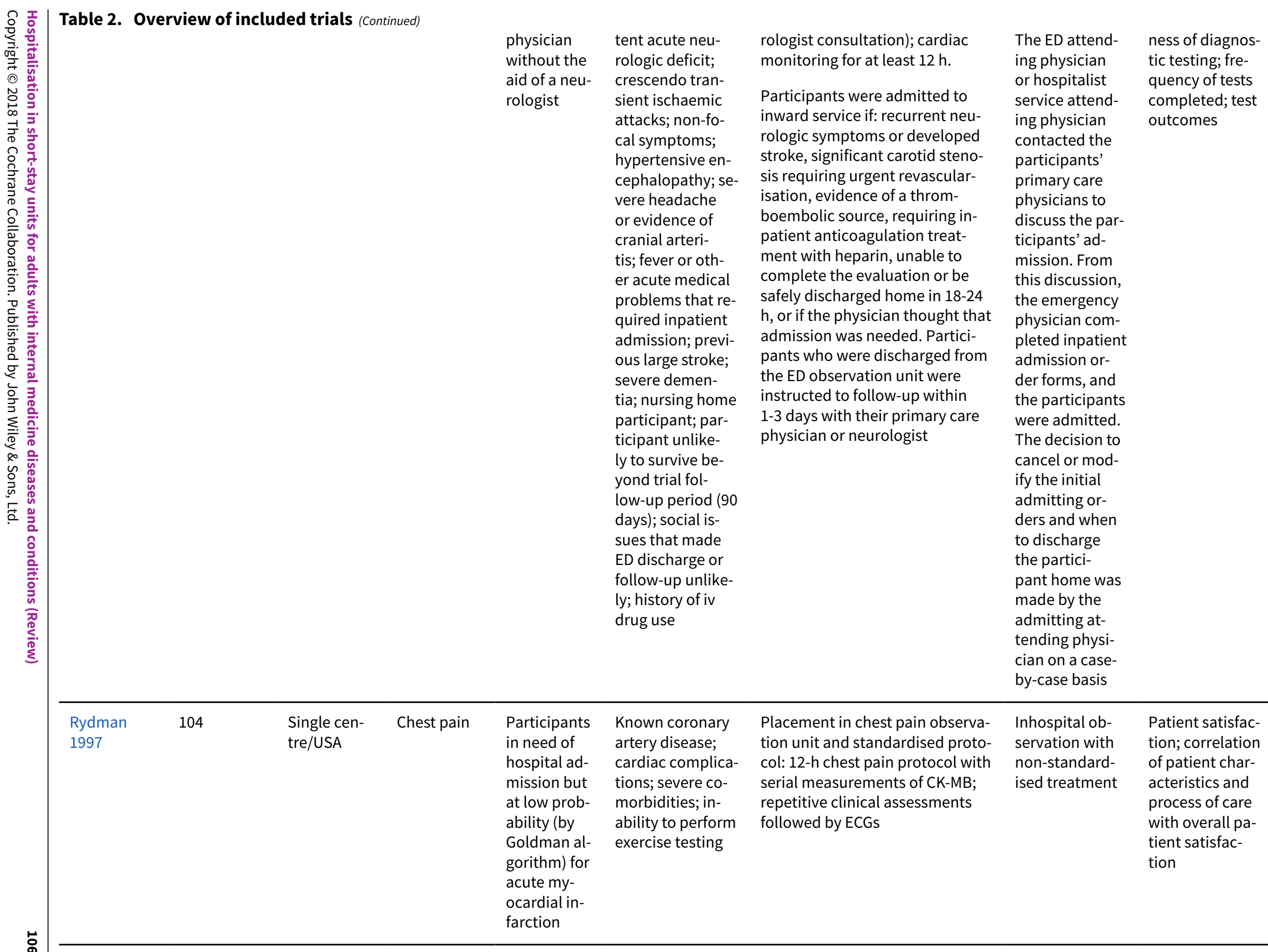




\begin{tabular}{|c|c|c|c|c|c|c|c|c|c|}
\hline Shen 2004 & 103 & $\begin{array}{l}\text { Single cen- } \\
\text { tre/USA }\end{array}$ & Syncope & $\begin{array}{l}\text { Age } \geq 18 \\
\text { years; resi- } \\
\text { dence in de- } \\
\text { fined near- } \\
\text { by coun- } \\
\text { ties; syn- } \\
\text { cope of un- } \\
\text { determined } \\
\text { cause; in- } \\
\text { termediate } \\
\text { risk for an } \\
\text { adverse car- } \\
\text { diovascular } \\
\text { outcome }\end{array}$ & $\begin{array}{l}\text { Identified cause } \\
\text { of syncope during } \\
\text { initial evaluation } \\
\text { in ED; any condi- } \\
\text { tion that would } \\
\text { require hospi- } \\
\text { tal admission; } \\
\text { non-syncope syn- } \\
\text { dromes }\end{array}$ & $\begin{array}{l}\text { Placement in ED syncope unit } \\
\text { and standardised protocol: con- } \\
\text { tinuous cardiac monitoring for up } \\
\text { to } 6 \text { h; hourly vital signs; ortho- } \\
\text { static blood pressure; echocar- } \\
\text { diography if participants had ab- } \\
\text { normal cardiovascular exami- } \\
\text { nation findings or an abnormal } \\
\text { ECG; tilt-table test in an electro- } \\
\text { physiological laboratory near the } \\
\text { syncope unit; electrophysiologi- } \\
\text { cal consultation upon request. If } \\
\text { tests or consultation could not be } \\
\text { performed while the participant } \\
\text { was in the syncope unit, arrange- } \\
\text { ments for an outpatient consul- } \\
\text { tation at a Heart Rhythm Centre; } \\
\text { tilt-table testing; or echocardio- } \\
\text { graphy could be made within } 72 \\
\text { h after dismissal from the unit. } \\
\text { An educational booklet on syn- } \\
\text { cope was given to each partici- } \\
\text { pant at the time of dismissal from } \\
\text { the syncope unit. The unit was } \\
\text { staffed by an ED physician and a } \\
\text { registered nurse }\end{array}$ & $\begin{array}{l}\text { Usual care: the } \\
\text { ED physician } \\
\text { was respon- } \\
\text { sible for mak- } \\
\text { ing the deci- } \\
\text { sion whether } \\
\text { further evalu- } \\
\text { ation was re- } \\
\text { quired and the } \\
\text { setting in which } \\
\text { the evaluation } \\
\text { should occur. } \\
\text { ED diagnostic } \\
\text { testing was per- } \\
\text { formed at the } \\
\text { discretion of } \\
\text { the physician } \\
\text { on the basis } \\
\text { of the partic- } \\
\text { ipant's initial } \\
\text { history. phys- } \\
\text { ical examina- } \\
\text { tion, and labo- } \\
\text { ratory findings }\end{array}$ & $\begin{array}{l}\text { Diagnostic } \\
\text { yield; hospital } \\
\text { admission rate; } \\
\text { net diagnostic } \\
\text { yield; length of } \\
\text { hospital stay at } \\
\text { the completion } \\
\text { of the evalua- } \\
\text { tion of the in- } \\
\text { dex event; all- } \\
\text { cause mortality; } \\
\text { recurrent syn- } \\
\text { cope during fol- } \\
\text { low-up }\end{array}$ & 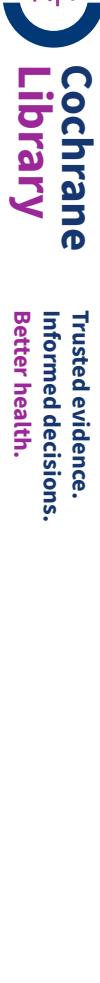 \\
\hline $\begin{array}{l}\text { Strøm 2017a } \\
\text { (Trial details } \\
\text { obtained } \\
\text { after con- } \\
\text { tact with au- } \\
\text { thors) }\end{array}$ & 430 & $\begin{array}{l}\text { Single cen- } \\
\text { tre/Den- } \\
\text { mark }\end{array}$ & $\begin{array}{l}\text { All internal } \\
\text { medicine } \\
\text { diseases or } \\
\text { conditions }\end{array}$ & $\begin{array}{l}\text { Age } \geq 75 \\
\text { years; acute } \\
\text { admission } \\
\text { to hospital } \\
\text { for an in- } \\
\text { ternal med- } \\
\text { icine dis- } \\
\text { ease; stable } \\
\text { (green-tag) } \\
\text { triage at the } \\
\text { time of ad- } \\
\text { mission }\end{array}$ & $\begin{array}{l}\text { Previous partici- } \\
\text { pation in the tri- } \\
\text { al, active partici- } \\
\text { pation in another } \\
\text { clinical trial, lack } \\
\text { of Danish civil } \\
\text { registration num- } \\
\text { ber, residency in } \\
\text { another country } \\
\text { than Denmark, } \\
\text { need of help get- } \\
\text { ting to the toi- } \\
\text { let in daily life, } \\
\text { no awareness of } \\
\text { the current date, } \\
\text { time and loca- } \\
\text { tion, no aware- } \\
\text { ness of name }\end{array}$ & $\begin{array}{l}\text { Placement in ED short-stay unit. } \\
\text { Discharge planning was initiat- } \\
\text { ed immediately after admission } \\
\text { to the short-stay unit. If the par- } \\
\text { ticipant needed further diagnos- } \\
\text { tic tests these were performed on } \\
\text { the same terms as in the ED, in- } \\
\text { cluding point-of-care ultrasonog- } \\
\text { raphy available around the clock, } \\
\text { acute blood samples analysed } \\
\text { in the ED's point-of-care labora- } \\
\text { tory from } 8 \text { am-10 pm, and sim- } \\
\text { ple X-rays in the ED's X-ray room } \\
\text { manned from } 10 \text { am- } 6 \text { pm. More } \\
\text { advanced diagnostic examina- } \\
\text { tions, such as CT or MRI scans } \\
\text { were performed at the Depart- } \\
\text { ment of Radiology on a fast-track }\end{array}$ & $\begin{array}{l}\text { Placement in } \\
\text { Internal Med- } \\
\text { icine Depart- } \\
\text { ment, no stan- } \\
\text { dardised treat- } \\
\text { ment protocols } \\
\text { were applied }\end{array}$ & $\begin{array}{l}\text { 90-day mortal- } \\
\text { ity, mortality } \\
\text { rate within the } \\
\text { full observa- } \\
\text { tion period, in- } \\
\text { hospital mor- } \\
\text { tality, adverse } \\
\text { events during } \\
\text { hospitalisation, } \\
\text { change in Law- } \\
\text { ton Instrumen- } \\
\text { tal Activities } \\
\text { of Daily Living } \\
\text { score within } 90 \\
\text { days from ad- } \\
\text { mission, in-hos- } \\
\text { pital length of } \\
\text { stay in hospi- }\end{array}$ & 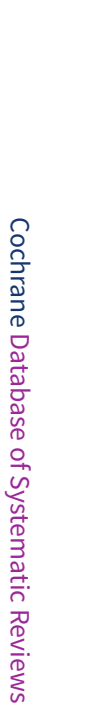 \\
\hline
\end{tabular}




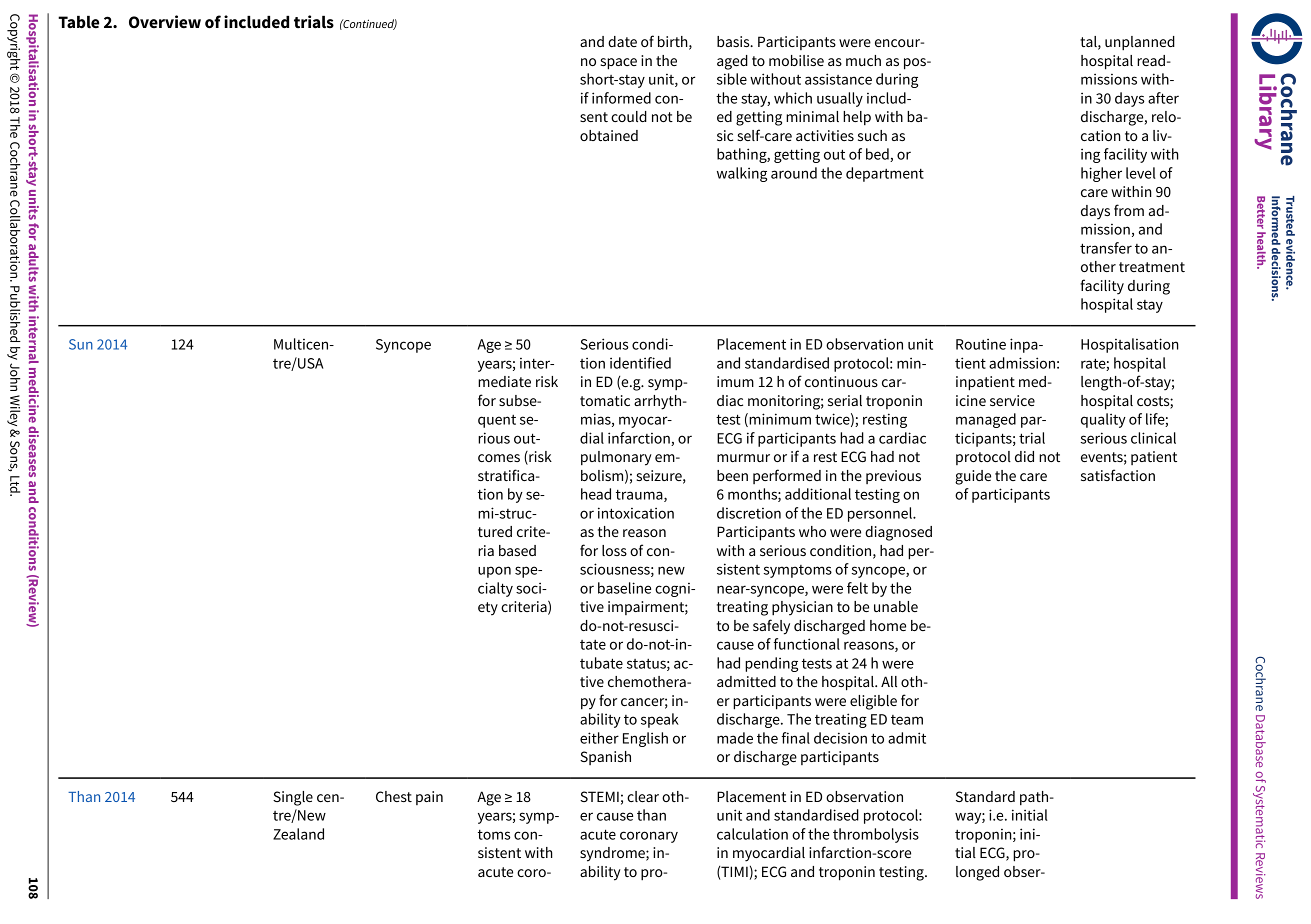


nary syn- vide consent;

drome;

physician

planned fur-

ther obser-

vation/tro-

ponin test-

ing for pos

sible acute

myocardial

infarction staff considered

recruitment to

be inappropri-

ate; chest pain

symptoms $>12 \mathrm{~h}$;

transfer from oth-

er hospital; preg-

nancy; previous

inclusion in tri-

al; inability to be

discharged after

hospitalisation
If TIMI = 0: placement in obser-

vation unit for $2 \mathrm{~h}$; repeated ECG

and troponin tests. If tests results

were negative, the participant

was discharged and scheduled to

a $72 \mathrm{~h}$ outpatient treadmill test. If

TIMI score was $>0$ or test results

were positive at any time, partici-

pants were admitted BP: Blood Pressure; bpm: beats per minute; CK-MB: Creatine Kinase Myocardial B-fraction; CT: Computerised Tomography scan; ECG: electrocardiogram; ED: Emergency
Department; h:hour; iv: intravenous; mmHg: Millimetres of Mercury; mV: Millivolt; MRI: magnetic resonance imaging scan: PaCO2: partial pressure of carbon dioxide in arterial blood; PaO2: partial pressure of oxygen in arterial blood; STEMI: ST-elevation myocardial infarction; SVT: supraventricular tachycardia; VT: ventricular tachycardia vation, second

troponin test

6-12 $\mathrm{h}$ after on-

set of pain, ob-

servation in in-

low-up appoint-

ments depend-

ed on the clini-

cian (usually 7-

day follow-u

with general

practitioner) ward unit, fol- 
Table 3. Contact with trialists

\begin{tabular}{|c|c|c|c|c|c|}
\hline Study ID & Contact information & $\begin{array}{l}\text { Date sent first in- } \\
\text { quiry }\end{array}$ & $\begin{array}{l}\text { Date reply re- } \\
\text { ceived }\end{array}$ & $\begin{array}{l}\text { Follow-up or } \\
\text { last reminder } \\
\text { sent }\end{array}$ & $\begin{array}{l}\text { Date reply re- } \\
\text { ceived }\end{array}$ \\
\hline Chivite 2008 & dchivite@bellvitgehospital.cat & 27 February 2017 & 6 March 2017 & $\begin{array}{l}27 \text { February } \\
2017\end{array}$ & \\
\hline Decker 2008 & decker.wyatt@mayo.edu & 27 February 2017 & $\begin{array}{l}27 \text { February } \\
2017\end{array}$ & $\begin{array}{l}27 \text { February } \\
2017\end{array}$ & \\
\hline Farkouh 1998 & $\begin{array}{l}\text { cullen.michael@mayo.edu; } \\
\text { michael.farkouh@mssm.edu }\end{array}$ & 27 February 2017 & No reply & 11 July 2017 & \\
\hline Gomez 1996 & Idjande3@gmail.com & 27 February 2017 & 2 March 2017 & $\begin{array}{l}27 \text { February } \\
2017\end{array}$ & 2 March 2017 \\
\hline $\begin{array}{l}\text { McDermott } \\
1997\end{array}$ & $\begin{array}{l}\text { robert.j.rydman@uic.edu; rrobert- } \\
\text { s@rush.edu }\end{array}$ & 28 February 2017 & No reply & 11 July 2017 & No reply \\
\hline Miller 2010 & cmiller@wakehealth.edu & 28 February 2017 & $\begin{array}{l}28 \text { February } \\
2017\end{array}$ & NA & No reply \\
\hline Miller 2013 & cmiller@wakehealth.edu & 28 February 2017 & $\begin{array}{l}28 \text { February } \\
2017\end{array}$ & NA & No reply \\
\hline Roberts 1997 & $\begin{array}{l}\text { robert.j.rydman@uic.edu; rrobert- } \\
\text { s@rush.edu }\end{array}$ & 28 February 2017 & No reply & 11 July 2017 & No reply \\
\hline Ross 2007 & mross@beaumont.edu & 28 February 2017 & No reply & 11 July 2017 & No reply \\
\hline Rydman 1997 & $\begin{array}{l}\text { robert.j.rydman@uic.edu; rrobert- } \\
\text { s@rush.edu }\end{array}$ & 28 February 2017 & No reply & 11 July 2017 & NA \\
\hline Shen 2004 & wshen@mayo.edu & 28 February 2017 & $\begin{array}{l}28 \text { February } \\
2017\end{array}$ & NA & 13 July 2017 \\
\hline Strøm 2017a & cstr@regionsjaelland.dk & 3 March 2017 & 3 March 2017 & NA & NA \\
\hline Sun 2014 & sunb@ohsu.edu & 28 February 2017 & $\begin{array}{l}28 \text { February } \\
2017\end{array}$ & NA & \\
\hline Than 2014 & $\begin{array}{l}\text { martin@thanstedman.onmi- } \\
\text { crosoft.com }\end{array}$ & 28 February 2017 & $\begin{array}{l}28 \text { February } \\
2017\end{array}$ & 11 July 2017 & \\
\hline
\end{tabular}

Table 4. Mortality at the time point closest to 90 days

\footnotetext{
This table was missing, and had to be replaced.
} 


\begin{tabular}{|c|c|c|c|c|c|c|c|c|}
\hline Study ID & $\begin{array}{l}\text { Dead in } \\
\text { short-stay } \\
\text { unit (n) }\end{array}$ & $\begin{array}{l}\text { Analysed } \\
\text { short-stay } \\
\text { unit (n) }\end{array}$ & $\begin{array}{l}\text { Ran- } \\
\text { domised } \\
\text { short-stay } \\
\text { unit (n) }\end{array}$ & $\begin{array}{l}\text { Dead in } \\
\text { usual-care } \\
\text { group }(\mathbf{n})\end{array}$ & $\begin{array}{l}\text { Analysed } \\
\text { usual-care } \\
\text { group }(\mathbf{n})\end{array}$ & $\begin{array}{l}\text { Ran- } \\
\text { domised } \\
\text { usual-care } \\
\text { group (n) }\end{array}$ & $\begin{array}{l}\text { 'Risk of } \\
\text { bias' judge- } \\
\text { ment }\end{array}$ & Time point \\
\hline Chivite 2008 & 5 & 65 & 70 & 3 & 62 & 69 & High & 90 days \\
\hline Decker 2008 & 0 & 75 & 75 & 0 & 78 & 78 & High & 6 months \\
\hline Farkouh 1998 & 17 & 204 & 212 & 17 & 203 & 212 & High & $\begin{array}{l}\text { Maximum observation time medi- } \\
\text { an } 5.5 \text { years (IQR } 4.8-6.0 \text { years) }\end{array}$ \\
\hline Gomez 1996 & 0 & 50 & 50 & 0 & 50 & 50 & High & 30 days \\
\hline McDermott 1997 & 0 & 110 & 110 & 1 & 112 & 112 & High & 8 weeks \\
\hline Miller 2010 & 0 & 52 & 53 & 0 & 57 & 57 & High & 1 year \\
\hline Miller 2013 & 0 & 52 & 52 & 0 & 53 & 53 & High & 90 days \\
\hline Roberts 1997 & 0 & 82 & 82 & 0 & 83 & 83 & High & 8 weeks \\
\hline Shen 2004 & 2 & 51 & 51 & 3 & 52 & 52 & High & 2 years \\
\hline Strøm 2017a & 42 & 208 & 215 & 55 & 210 & 215 & High & $\begin{array}{l}\text { Maximum observation time ranged } \\
\text { between } 90 \text { and } 641 \text { days per par- } \\
\text { ticipant }\end{array}$ \\
\hline Sun 2014 & 0 & 62 & 62 & 0 & 62 & 62 & High & Within admission \\
\hline Than 2014 & 0 & 270 & 271 & 0 & 272 & 273 & High & 30 days \\
\hline
\end{tabular}

Table 6. Serious adverse events (SAEs) at the time point closest to 90 days

\begin{tabular}{|c|c|c|c|c|c|c|c|c|c|}
\hline Study ID & Definition & $\begin{array}{l}\text { Partic- } \\
\text { ipants } \\
\text { with SAE } \\
\text { in short- }\end{array}$ & $\begin{array}{l}\text { Partic- } \\
\text { ipants } \\
\text { analysed } \\
\text { short-stay } \\
\text { unit (n) }\end{array}$ & $\begin{array}{l}\text { Partici- } \\
\text { pants ran- } \\
\text { domised } \\
\text { short-stay } \\
\text { unit (n) }\end{array}$ & $\begin{array}{l}\text { Partic- } \\
\text { ipants } \\
\text { with SAE } \\
\text { in usu- }\end{array}$ & $\begin{array}{l}\text { Partici- } \\
\text { pants ran- } \\
\text { domised } \\
\text { analysed } \\
\text { usu- }\end{array}$ & $\begin{array}{l}\text { Partici- } \\
\text { pants ran- } \\
\text { domised } \\
\text { usu- }\end{array}$ & $\begin{array}{l}\text { 'Risk of bias' } \\
\text { judgement }\end{array}$ & $\begin{array}{l}\text { Time } \\
\text { point }\end{array}$ \\
\hline
\end{tabular}




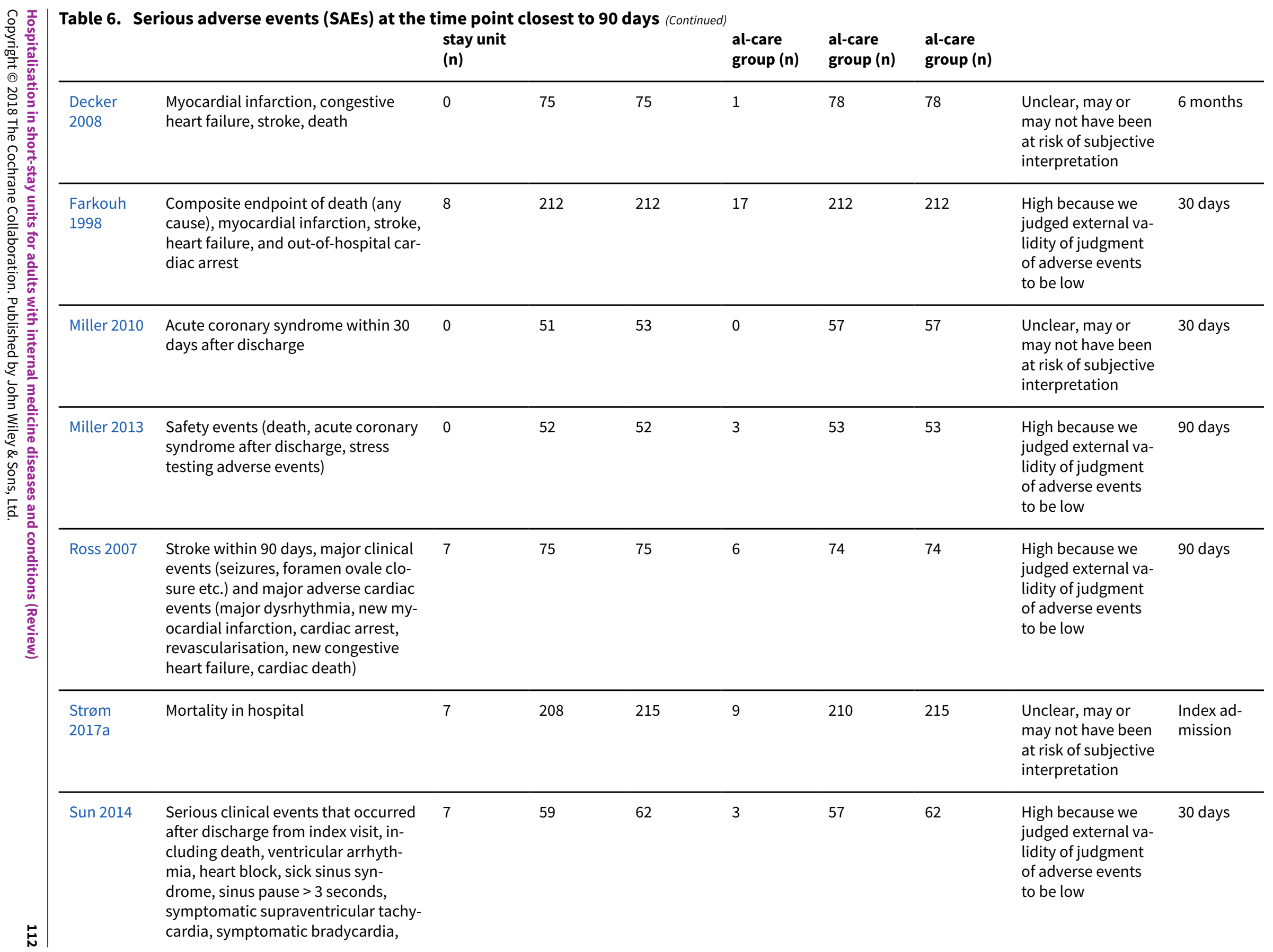




\begin{tabular}{|c|c|c|c|c|c|c|c|c|c|}
\hline 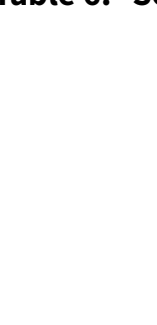 & $\begin{array}{l}\text { major cardiac intervention, myocar- } \\
\text { dial infarction, stroke, pulmonary } \\
\text { embolism, aortic dissection, non- } \\
\text { traumatic intracranial haemorrhage, } \\
\text { internal haemorrhage or anaemia re- } \\
\text { quiring transfusion, and major trau- } \\
\text { matic injury associated with syn- } \\
\text { cope, near-syncope or fall }\end{array}$ & & & & & & & & \\
\hline Than 2014 & $\begin{array}{l}\text { Major adverse cardiac event: cardiac } \\
\text { death, cardiac arrest, emergency } \\
\text { revascularisation procedure, cardio- } \\
\text { genic shock, ventricular arrhythmia } \\
\text { needing intervention, high-degree } \\
\text { atrioventricular block needing inter- } \\
\text { vention, or myocardial infarction }\end{array}$ & 47 & 270 & 271 & 35 & 272 & 273 & $\begin{array}{l}\text { High because we } \\
\text { judged external va- } \\
\text { lidity of judgment } \\
\text { of adverse events } \\
\text { to be low }\end{array}$ & 30 days \\
\hline \multicolumn{10}{|c|}{ n: number of participants; SAE: serious adverse event } \\
\hline \multicolumn{10}{|c|}{ Table 7. Serious adverse events (SAEs) at maximum follow-up } \\
\hline Study ID & Definition & $\begin{array}{l}\text { Partic- } \\
\text { ipants } \\
\text { with SAE } \\
\text { in short- } \\
\text { stay unit } \\
\text { (n) }\end{array}$ & $\begin{array}{l}\text { Partic- } \\
\text { ipants } \\
\text { analysed } \\
\text { short-stay } \\
\text { unit (n) }\end{array}$ & $\begin{array}{l}\text { Partici- } \\
\text { pants ran- } \\
\text { domised } \\
\text { short-stay } \\
\text { unit (n) }\end{array}$ & $\begin{array}{l}\text { Partic- } \\
\text { ipants } \\
\text { with SAE } \\
\text { in usu- } \\
\text { al-care } \\
\text { group(n) }\end{array}$ & $\begin{array}{l}\text { Partici- } \\
\text { pants ran- } \\
\text { domised } \\
\text { analysed } \\
\text { usu- } \\
\text { al-care } \\
\text { group(n) }\end{array}$ & $\begin{array}{l}\text { Partici- } \\
\text { pants ran- } \\
\text { domised } \\
\text { usu- } \\
\text { al-care } \\
\text { group(n) }\end{array}$ & $\begin{array}{l}\text { Risk of bias out- } \\
\text { come }\end{array}$ & $\begin{array}{l}\text { Time- } \\
\text { point }\end{array}$ \\
\hline $\begin{array}{l}\text { Decker } \\
2008\end{array}$ & $\begin{array}{l}\text { Myocardial infarction, congestive } \\
\text { heart failure, stroke, death }\end{array}$ & 0 & 75 & 75 & 1 & 78 & 78 & $\begin{array}{l}\text { Unclear, may or } \\
\text { may not have been } \\
\text { at risk of subjective } \\
\text { interpretation }\end{array}$ & 6 months \\
\hline $\begin{array}{l}\text { Farkouh } \\
1998\end{array}$ & $\begin{array}{l}\text { Composite endpoint of death (any } \\
\text { cause), myocardial infarction, stroke, } \\
\text { heart failure, and out-of-hospital car- } \\
\text { diac arrest }\end{array}$ & 44 & 204 & 212 & 41 & 203 & 212 & $\begin{array}{l}\text { High because we } \\
\text { judged external va- } \\
\text { lidity of judgment } \\
\text { of adverse events } \\
\text { to be low }\end{array}$ & $\begin{array}{l}\text { Maximum } \\
\text { observa- } \\
\text { tion time } \\
\text { median } \\
5,5 \text { years } \\
\text { (IQR 4.8-6 } \\
\text { years) }\end{array}$ \\
\hline
\end{tabular}




\begin{tabular}{|c|c|c|c|c|c|c|c|c|c|}
\hline Miller 2010 & $\begin{array}{l}\text { Acute coronary syndrome within } 30 \\
\text { days after discharge }\end{array}$ & 3 & 52 & 53 & 5 & 57 & 57 & $\begin{array}{l}\text { Unclear, may or } \\
\text { may not have been } \\
\text { at risk of subjective } \\
\text { interpretation }\end{array}$ & 30 days \\
\hline Miller 2013 & $\begin{array}{l}\text { Safety events (death, acute coronary } \\
\text { syndrome after discharge, stress } \\
\text { testing adverse events) }\end{array}$ & 0 & 52 & 52 & 3 & 53 & 53 & $\begin{array}{l}\text { High because we } \\
\text { judged external va- } \\
\text { lidity of judgment } \\
\text { of adverse events } \\
\text { to be low }\end{array}$ & 90 days \\
\hline Ross 2007 & $\begin{array}{l}\text { Stroke within } 90 \text { days, major clinical } \\
\text { events (seizures, foramen ovale clo- } \\
\text { sure etc.) and major adverse cardiac } \\
\text { events (major dysrhythmia, new my- } \\
\text { ocardial infarction, cardiac arrest, } \\
\text { revascularisation, new congestive } \\
\text { heart failure, cardiac death) }\end{array}$ & 7 & 75 & 75 & 6 & 74 & 74 & $\begin{array}{l}\text { High because we } \\
\text { judged external va- } \\
\text { lidity of judgment } \\
\text { of adverse events } \\
\text { to be low }\end{array}$ & 90 days \\
\hline $\begin{array}{l}\text { Strøm } \\
2017 a\end{array}$ & Mortality in-hospital & 7 & 208 & 215 & 9 & 210 & 215 & $\begin{array}{l}\text { Unclear, may or } \\
\text { may not have been } \\
\text { at risk of subjective } \\
\text { interpretation }\end{array}$ & $\begin{array}{l}\text { Index ad- } \\
\text { mission }\end{array}$ \\
\hline Sun 2014 & $\begin{array}{l}\text { Serious clinical events that occurred } \\
\text { after discharge from index visit, in- } \\
\text { cluding death, ventricular arrhyth- } \\
\text { mia, heart block, sick sinus syn- } \\
\text { drome, sinus pause > } 3 \text { seconds, } \\
\text { symptomatic supraventricular tachy- } \\
\text { cardia, symptomatic bradycardia, } \\
\text { major cardiac intervention, myocar- } \\
\text { dial infarction, stroke, pulmonary } \\
\text { embolism, aortic dissection, non- } \\
\text { traumatic intracranial haemorrhage, } \\
\text { internal haemorrhage or anaemia re- } \\
\text { quiring transfusion, and major trau- } \\
\text { matic injury associated with syn- } \\
\text { cope, near-syncope or fall }\end{array}$ & 9 & 53 & 62 & 8 & 52 & 62 & $\begin{array}{l}\text { High because we } \\
\text { judged external va- } \\
\text { lidity of judgment } \\
\text { of adverse events } \\
\text { to be low }\end{array}$ & 6 months \\
\hline Than 2014 & $\begin{array}{l}\text { Major adverse cardiac event: cardiac } \\
\text { death, cardiac arrest, emergency } \\
\text { revascularisation procedure, cardio- } \\
\text { genic shock, ventricular arrhythmia }\end{array}$ & 47 & 270 & 271 & 35 & 272 & 273 & $\begin{array}{l}\text { High because we } \\
\text { judged external va- } \\
\text { lidity of judgment }\end{array}$ & 30 days \\
\hline
\end{tabular}




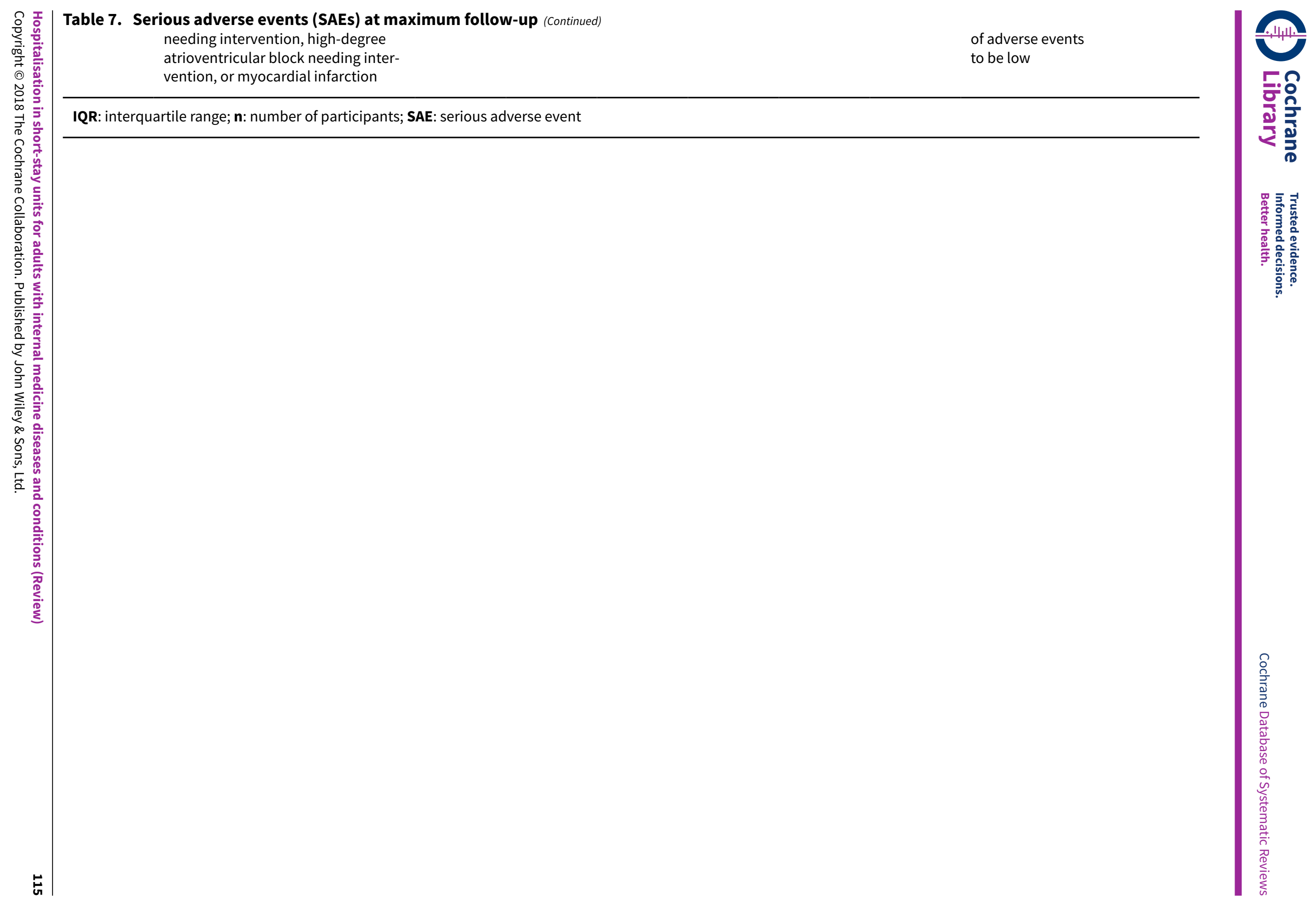


Table 8. Quality of life

\begin{tabular}{|c|c|c|c|c|c|}
\hline \multirow[t]{2}{*}{ Study ID } & \multirow[t]{2}{*}{ Scale } & \multirow{2}{*}{$\begin{array}{l}\text { Elaboration of scale } \\
\text { used }\end{array}$} & \multirow{2}{*}{$\begin{array}{l}\text { Reason for } \\
\text { admission }\end{array}$} & \multicolumn{2}{|c|}{ Time-point of assessment, type of score } \\
\hline & & & & $\begin{array}{l}\text { Participants } \\
\text { evaluat- } \\
\text { ed (n)/ran- } \\
\text { domised } \\
\text { per group (n) }\end{array}$ & $\begin{array}{l}\text { Outcomes as reported in trial } \\
\text { paper }\end{array}$ \\
\hline \multirow[t]{8}{*}{ Chivite 2008} & \multirow{8}{*}{$\begin{array}{l}\text { Minnesota Liv- } \\
\text { ing With Heart } \\
\text { Failure Quality } \\
\text { of Life Scale }\end{array}$} & \multirow{8}{*}{$\begin{array}{l}\text { Patient self-assessment } \\
\text { of how heart failure af- } \\
\text { fects participants' daily } \\
\text { life, } 21 \text { items. Each item } \\
\text { assessed on a 5-point } \\
\text { Likert scale from } 1 \text { ('com- } \\
\text { pletely agree') to } 5 \text { ('com- } \\
\text { pletely disagree'). Total } \\
\text { score ranges from 5-105 } \\
\text { (from best to worst) }\end{array}$} & \multirow[t]{8}{*}{ Acute illness } & \multicolumn{2}{|c|}{ Mean end-score (SD) at 3 months } \\
\hline & & & & $\begin{array}{l}\text { Short-stay } \\
\text { unit: } 59 / 70\end{array}$ & Short-stay unit: 23 (9) \\
\hline & & & & $\begin{array}{l}\text { Usual care: } \\
59 / 69\end{array}$ & Usual care: 22 (9) \\
\hline & & & & Difference & Mean difference: not provided \\
\hline & & & & \multicolumn{2}{|c|}{ Mean end-score (SD) at 12 months } \\
\hline & & & & $\begin{array}{l}\text { Short-stay } \\
\text { unit: } 48 / 70\end{array}$ & Short-stay unit: 22 (12) \\
\hline & & & & $\begin{array}{l}\text { Usual care: } \\
47 / 69\end{array}$ & Usual care: 22 (12) \\
\hline & & & & Difference & Mean difference: not provided \\
\hline Chivite 2008 & $\begin{array}{l}\text { European } \\
\text { Heart Failure } \\
\text { Self-Care Be- } \\
\text { haviour Scale }\end{array}$ & $\begin{array}{l}9 \text { items grouped in } 2 \text { di- } \\
\text { mensions: consulting be- } \\
\text { haviours and adherence } \\
\text { with treatment regimen. } \\
\text { Each item assessed on a } \\
5 \text {-point Likert scale from } \\
1 \text { ('completely agree') to } \\
5 \text { ('completely disagree'). } \\
\text { Total score ranges from } \\
9-45\end{array}$ & Acute illness & $\begin{array}{l}\text { Numbers not } \\
\text { provided }\end{array}$ & Numbers not provided \\
\hline \multirow{3}{*}{$\begin{array}{l}\text { McDermott } \\
1997\end{array}$} & \multirow{3}{*}{$\begin{array}{l}\text { The Short } \\
\text { Form-36 } \\
\text { Health Survey }\end{array}$} & \multirow{3}{*}{$\begin{array}{l}\text { 8-scale score system. } \\
\text { Each scale is directly } \\
\text { transformed into a 0-100 } \\
\text { scale on the assumption } \\
\text { that each question car- } \\
\text { ries equal weight. The } \\
\text { lower the score the more } \\
\text { disability }\end{array}$} & \multirow[t]{3}{*}{ Asthma } & \multicolumn{2}{|c|}{ Mean end-score (SD) at 1 week } \\
\hline & & & & $\begin{array}{l}\text { Short-stay } \\
\text { unit: } 57 / 110\end{array}$ & $\begin{array}{l}\text { Short-stay unit: physical func- } \\
\text { tioning } 72 \text { ( } 27.43) \text {; physical func- } \\
\text { tioning role } 48 \text { (43.00); emotion- } \\
\text { al functioning role } 78 \text { (51.31); so- } \\
\text { cial functioning } 80 \text { (27.95); bodily } \\
\text { pain } 78 \text { (28.10); mental health } 78 \\
\text { (19.44); vitality } 59 \text { (22.99); general } \\
\text { health perceptions } 48 \text { (23.05) }\end{array}$ \\
\hline & & & & $\begin{array}{l}\text { Usual care: } \\
56 / 112\end{array}$ & $\begin{array}{l}\text { Usual care: physical function- } \\
\text { ing } 58 \text { ( } 29.04) \text {; physical function- } \\
\text { ing role } 35 \text { ( } 43.09) \text {; emotional } \\
\text { functioning role } 45 \text { ( } 44.62) \text {; so- } \\
\text { cial functioning } 68 \text { ( } 29.27) \text {; bodily } \\
\text { pain } 74 \text { (29.52); mental health } 67\end{array}$ \\
\hline
\end{tabular}


Table 8. Quality of life (Continued)

(26.00); vitality 47 (25.73); general health perceptions $47(19.60)$

\begin{tabular}{|c|c|c|c|c|c|}
\hline \multirow[t]{4}{*}{ Sun 2014} & \multirow{4}{*}{$\begin{array}{l}\text { The Syncope } \\
\text { Functional } \\
\text { Status Ques- } \\
\text { tionnaire for } \\
\text { symptom-spe- } \\
\text { cific quality of } \\
\text { life }\end{array}$} & \multirow{4}{*}{$\begin{array}{l}\text { Ranges from } 0-100 ; 0 \text { in- } \\
\text { dicates no syncope-relat- } \\
\text { ed impairment and } 100 \\
\text { indicates maximum im- } \\
\text { pairment }\end{array}$} & \multirow[t]{4}{*}{ Syncope } & \multicolumn{2}{|c|}{ Mean change score (SD) at 1 month } \\
\hline & & & & $\begin{array}{l}\text { Short-stay } \\
\text { unit: } 48 / 62\end{array}$ & Short-stay unit: $-7.6(20.1)$ \\
\hline & & & & $\begin{array}{l}\text { Usual care: } \\
41 / 62\end{array}$ & Usual care: -2.4 (26.3) \\
\hline & & & & Difference & $\begin{array}{l}\text { Mean difference: }-5.2(95 \% \mathrm{Cl} \\
-15.2 ; 4.8)\end{array}$ \\
\hline \multirow[t]{4}{*}{ Sun 2014} & \multirow{4}{*}{$\begin{array}{l}\text { Change in } \\
\text { Quality of Well } \\
\text { Being Scale } \\
\text { for general } \\
\text { health utility }\end{array}$} & \multirow{4}{*}{$\begin{array}{l}\text { Ranges from } 0-1 ; 0 \text { indi- } \\
\text { cates the worst possible } \\
\text { health and } 1 \text { indicates } \\
\text { optimum health }\end{array}$} & \multirow[t]{4}{*}{ Syncope } & \multicolumn{2}{|c|}{ Mean change score (SD) at 1 month } \\
\hline & & & & $\begin{array}{l}\text { Short-stay } \\
\text { unit: } 48 / 62\end{array}$ & Short-stay unit: $0.00(0.20)$ \\
\hline & & & & $\begin{array}{l}\text { Usual care: } \\
41 / 62\end{array}$ & Usual care: $0.03(0.18)$ \\
\hline & & & & Difference & $\begin{array}{l}\text { Mean difference: }-0.02(95 \% \mathrm{Cl} \\
-0.10 ; 0.06)\end{array}$ \\
\hline \multirow[t]{4}{*}{ Than 2014} & \multirow{4}{*}{$\begin{array}{l}\text { EuroQol-5 Do- } \\
\text { main }\end{array}$} & \multirow{4}{*}{$\begin{array}{l}\text { Descriptive system of } \\
\text { health-related quality-of- } \\
\text { life states, consisting of } \\
5 \text { dimensions (mobility, } \\
\text { self-care, usual activities, } \\
\text { pain/discomfort, anxi- } \\
\text { ety/depression) }\end{array}$} & \multirow[t]{4}{*}{ Chest pain } & \multicolumn{2}{|c|}{ Mean health utility end-score (SD) at 3 months } \\
\hline & & & & $\begin{array}{l}\text { Short-stay } \\
\text { unit: } 253 / 470\end{array}$ & Short-stay unit: 0.716 (SD 0.109) \\
\hline & & & & $\begin{array}{l}\text { Usual care: } \\
250 / 272\end{array}$ & Usual care: 0.728 (SD 0.093) \\
\hline & & & & Notes & $\begin{array}{l}\text { We obtained data through con- } \\
\text { tact with trialists. We imputed } \\
\text { missing data; for each participant } \\
\text { without missing data for any one } \\
\text { of the EQ-5D dimensions, we cal- } \\
\text { culated a tariff value based on } \\
\text { the } 1999 \text { New Zealand tariff data } \\
\text { of a healthy population }\end{array}$ \\
\hline
\end{tabular}

CI: confidence interval; EQ-5D: EuroQol-5 Domain; n: number of participants; SD: standard deviation 


\begin{tabular}{|c|c|c|c|c|c|c|c|c|c|}
\hline Study ID & Definition & $\begin{array}{l}\text { Partic- } \\
\text { ipants } \\
\text { with read- } \\
\text { mission in } \\
\text { short-stay } \\
\text { unit (n) }\end{array}$ & $\begin{array}{l}\text { Partic- } \\
\text { ipants } \\
\text { analysed } \\
\text { short-stay } \\
\text { unit (n) }\end{array}$ & $\begin{array}{l}\text { Partici- } \\
\text { pants ran- } \\
\text { domised } \\
\text { short-stay } \\
\text { unit (n) }\end{array}$ & $\begin{array}{l}\text { Partic- } \\
\text { ipants } \\
\text { with read- } \\
\text { mission } \\
\text { in usu- } \\
\text { al-care } \\
\text { group(n) }\end{array}$ & $\begin{array}{l}\text { Partici- } \\
\text { pants ran- } \\
\text { domised } \\
\text { analysed } \\
\text { usu- } \\
\text { al-care } \\
\text { group(n) }\end{array}$ & $\begin{array}{l}\text { Partici- } \\
\text { pants ran- } \\
\text { domised } \\
\text { usu- } \\
\text { al-care } \\
\text { group(n) }\end{array}$ & 'Risk of bias' judgement & $\begin{array}{l}\text { Time } \\
\text { point }\end{array}$ \\
\hline $\begin{array}{l}\text { Chivite } \\
2008\end{array}$ & $\begin{array}{l}\text { Hospital readmission with } \\
\text { a main diagnosis of acute } \\
\text { heart failure }\end{array}$ & 19 & 65 & 70 & 14 & 62 & 69 & $\begin{array}{l}\text { Unclear, may or may not have } \\
\text { been at risk of subjective in- } \\
\text { terpretation }\end{array}$ & 90 days \\
\hline $\begin{array}{l}\text { Decker } \\
2008\end{array}$ & $\begin{array}{l}\text { Hospital readmission, any } \\
\text { type }\end{array}$ & 25 & 75 & 75 & 27 & 78 & 78 & Low, objective measurement & 6 months \\
\hline $\begin{array}{l}\text { Farkouh } \\
1998\end{array}$ & $\begin{array}{l}\text { Hospital readmission to } \\
\text { cardiac care }\end{array}$ & $?$ & $?$ & 212 & $?$ & $?$ & 212 & $\begin{array}{l}\text { Unclear, may or may not have } \\
\text { been at risk of subjective in- } \\
\text { terpretation }\end{array}$ & 6 months \\
\hline Miller 2010 & $\begin{array}{l}\text { Cardiac-related hospital } \\
\text { readmission }\end{array}$ & 0 & 53 & 53 & 3 & 57 & 57 & $\begin{array}{l}\text { Unclear, may or may not have } \\
\text { been at risk of subjective in- } \\
\text { terpretation }\end{array}$ & 30 days \\
\hline Miller 2013 & $\begin{array}{l}\text { Hospital readmission, any } \\
\text { type }\end{array}$ & 4 & 52 & 52 & 12 & 53 & 53 & Low, objective measurement & 90 days \\
\hline $\begin{array}{l}\text { Roberts } \\
1997\end{array}$ & $\begin{array}{l}\text { Hospital readmission, any } \\
\text { type }\end{array}$ & 5 & 82 & 82 & 4 & 83 & 83 & Low, objective measurement & 8 weeks \\
\hline Ross 2007 & $\begin{array}{l}\text { Hospital readmission or } \\
\text { revisit, not entirely clear }\end{array}$ & 9 & 75 & 75 & 9 & 74 & 74 & $\begin{array}{l}\text { Unclear, may or may not have } \\
\text { been at risk of subjective in- } \\
\text { terpretation }\end{array}$ & 90 days \\
\hline $\begin{array}{l}\text { Strøm } \\
2017 a\end{array}$ & $\begin{array}{l}\text { Hospital readmission, any } \\
\text { type }\end{array}$ & 26 & 201 & 215 & 58 & 201 & 215 & Low, objective measurement & 30 days \\
\hline Than 2014 & $\begin{array}{l}\text { Hospital readmission, any } \\
\text { type }\end{array}$ & 22 & 270 & 271 & 20 & 272 & 273 & Low, objective measurement & 30 days \\
\hline
\end{tabular}




\begin{tabular}{|c|c|c|c|c|c|c|c|c|c|}
\hline Study ID & Definition & $\begin{array}{l}\text { Partic- } \\
\text { ipants } \\
\text { with read- } \\
\text { mission in } \\
\text { short-stay } \\
\text { unit (n) }\end{array}$ & $\begin{array}{l}\text { Partic- } \\
\text { ipants } \\
\text { analysed } \\
\text { short-stay } \\
\text { unit (n) }\end{array}$ & $\begin{array}{l}\text { Partici- } \\
\text { pants ran- } \\
\text { domised } \\
\text { short-stay } \\
\text { unit (n) }\end{array}$ & $\begin{array}{l}\text { Partic- } \\
\text { ipants } \\
\text { with read- } \\
\text { mission } \\
\text { in usu- } \\
\text { al-care } \\
\text { group(n) }\end{array}$ & $\begin{array}{l}\text { Partici- } \\
\text { pants ran- } \\
\text { domised } \\
\text { analysed } \\
\text { usu- } \\
\text { al-care } \\
\text { group(n) }\end{array}$ & $\begin{array}{l}\text { Partici- } \\
\text { pants ran- } \\
\text { domised } \\
\text { usu- } \\
\text { al-care } \\
\text { group(n) }\end{array}$ & Risk of bias outcome & $\begin{array}{l}\text { Time } \\
\text { point }\end{array}$ \\
\hline $\begin{array}{l}\text { Chivite } \\
2008\end{array}$ & $\begin{array}{l}\text { Hospital readmission with } \\
\text { a main diagnosis of acute } \\
\text { heart failure }\end{array}$ & 25 & 53 & 70 & 21 & 53 & 69 & Low, objective measurement & 12 months \\
\hline $\begin{array}{l}\text { Decker } \\
2008\end{array}$ & $\begin{array}{l}\text { Hospital readmission, any } \\
\text { type }\end{array}$ & 25 & 75 & 75 & 27 & 78 & 78 & $\begin{array}{l}\text { Unclear, may or may not have } \\
\text { been at risk of subjective in- } \\
\text { terpretation }\end{array}$ & 6 months \\
\hline $\begin{array}{l}\text { Farkouh } \\
1998\end{array}$ & $\begin{array}{l}\text { Hospital readmission to } \\
\text { cardiac care }\end{array}$ & $?$ & $?$ & 212 & $?$ & $?$ & 212 & $\begin{array}{l}\text { Unclear, may or may not have } \\
\text { been at risk of subjective in- } \\
\text { terpretation }\end{array}$ & Unclear \\
\hline Miller 2010 & $\begin{array}{l}\text { Cardiac-related hospital } \\
\text { readmission }\end{array}$ & 6 & 52 & 53 & 20 & 57 & 57 & $\begin{array}{l}\text { Unclear, may or may not have } \\
\text { been at risk of subjective in- } \\
\text { terpretation }\end{array}$ & 1 year \\
\hline Miller 2013 & $\begin{array}{l}\text { Hospital readmission, any } \\
\text { type }\end{array}$ & 4 & 52 & 52 & 12 & 53 & 53 & Low, objective measurement & 90 days \\
\hline $\begin{array}{l}\text { Roberts } \\
1997\end{array}$ & $\begin{array}{l}\text { Hospital readmission, any } \\
\text { type }\end{array}$ & 5 & 82 & 82 & 4 & 83 & 83 & Low, objective measurement & 8 weeks \\
\hline Ross 2007 & $\begin{array}{l}\text { Hospital readmission or } \\
\text { revisit, not entirely clear }\end{array}$ & 9 & 75 & 75 & 9 & 74 & 74 & $\begin{array}{l}\text { Unclear, may or may not have } \\
\text { been at risk of subjective in- } \\
\text { terpretation }\end{array}$ & 90 days \\
\hline $\begin{array}{l}\text { Strøm } \\
2017 a\end{array}$ & $\begin{array}{l}\text { Hospital readmission, any } \\
\text { type }\end{array}$ & 26 & 201 & 215 & 58 & 201 & 215 & Low, objective measurement & 30 days \\
\hline Than 2014 & $\begin{array}{l}\text { Hospital readmission, any } \\
\text { type }\end{array}$ & 22 & 270 & 271 & 20 & 272 & 273 & Low, objective measurement & 30 days \\
\hline
\end{tabular}


Table 11. Length of stay in hospital

\begin{tabular}{|c|c|c|c|c|c|c|c|c|c|}
\hline Study & $\begin{array}{l}\text { Reason } \\
\text { for admis- } \\
\text { sion }\end{array}$ & $\begin{array}{l}\text { Partici- } \\
\text { pants eval- } \\
\text { uated/ran- } \\
\text { domised in } \\
\text { short-stay } \\
\text { group }(\mathrm{n} / \mathrm{n})\end{array}$ & $\begin{array}{l}\text { Length } \\
\text { of stay } \\
\text { in hours, } \\
\text { mean (SD) }\end{array}$ & $\begin{array}{l}\text { Length } \\
\text { of stay } \\
\text { in hours, } \\
\text { median } \\
\text { (IQR) }\end{array}$ & $\begin{array}{l}\text { Partic- } \\
\text { ipants } \\
\text { evaluat- } \\
\text { ed/ran- } \\
\text { domised } \\
\text { in usu- } \\
\text { al-care } \\
\text { group (n/ } \\
\text { n) }\end{array}$ & $\begin{array}{l}\text { Length } \\
\text { of stay } \\
\text { in hours, } \\
\text { mean (SD) }\end{array}$ & $\begin{array}{l}\text { Length } \\
\text { of stay } \\
\text { in hours, } \\
\text { median } \\
\text { (IQR) }\end{array}$ & Risk of bias for outcome & Notes \\
\hline $\begin{array}{l}\text { Decker } \\
2008\end{array}$ & $\begin{array}{l}\text { Atrial fib- } \\
\text { rillation }\end{array}$ & $75 / 75$ & $\begin{array}{l}12.6 \text { (un- } \\
\text { clear) }\end{array}$ & $\begin{array}{l}10.1 \text { (un- } \\
\text { clear) }\end{array}$ & $78 / 78$ & $\begin{array}{l}50.1 \text { (un- } \\
\text { clear) }\end{array}$ & $\begin{array}{l}25.2 \text { (un- } \\
\text { clear) }\end{array}$ & $\begin{array}{l}\text { Low due to objective mea- } \\
\text { surement }\end{array}$ & \\
\hline $\begin{array}{l}\text { Farkouh } \\
1998\end{array}$ & Chest pain & unclear/212 & 9.2 (unclear) & & $\begin{array}{l}\text { un- } \\
\text { clear/212 }\end{array}$ & unclear & & $\begin{array}{l}\text { Low due to objective mea- } \\
\text { surement }\end{array}$ & \\
\hline $\begin{array}{l}\text { McDer- } \\
\text { mott } 1997\end{array}$ & Asthma & $\begin{array}{l}\text { Short-stay } \\
\text { unit report- } \\
\text { ed by } 2 \text { sub- } \\
\text { groups } \\
\text { 1) Discharged } \\
\text { home 65/110 } \\
\text { 2) Admitted } \\
\text { to hospital } \\
45 / 110\end{array}$ & $\begin{array}{l}\text { 1) Dis- } \\
\text { charged } \\
\text { home } 8.8 \\
(3.6) \\
\text { 2) Admitted } \\
\text { to hospital } \\
77.0(43.5)\end{array}$ & NA & $112 / 112$ & $59.0(35.9)$ & NA & $\begin{array}{l}\text { Low due to objective mea- } \\
\text { surement }\end{array}$ & $\begin{array}{l}\text { Length of stay in } \\
\text { hospital was mea- } \\
\text { sured at different } \\
\text { time points for in- } \\
\text { tervention group, ei- } \\
\text { ther discharge from } \\
\text { short-stay unit facil- } \\
\text { ity or from time of } \\
\text { discharge from hos- } \\
\text { pital }\end{array}$ \\
\hline Miller 2010 & Chest pain & $53 / 53$ & $31.4(31.3)$ & $\begin{array}{l}25.7 \\
(20.7-31.3)\end{array}$ & $57 / 57$ & $36.6(22.9)$ & $\begin{array}{l}29.9 \\
(26.7-35.7)\end{array}$ & $\begin{array}{l}\text { Low due to objective mea- } \\
\text { surement }\end{array}$ & \\
\hline Miller 2013 & Chest pain & $52 / 52$ & $26.6(16.9)$ & $\begin{array}{l}21.1 \\
(14.8-25.2)\end{array}$ & $53 / 53$ & $51(65.6)$ & $\begin{array}{l}26.3 \\
(22.7-44.8)\end{array}$ & $\begin{array}{l}\text { Low due to objective mea- } \\
\text { surement }\end{array}$ & \\
\hline
\end{tabular}




\begin{tabular}{|c|c|c|c|c|c|c|c|c|c|c|c|}
\hline Ross 2007 & $\begin{array}{l}\text { Transient } \\
\text { ischaemic } \\
\text { attack }\end{array}$ & $75 / 75$ & & $\begin{array}{l}25.6 \\
(21.9-28.7)\end{array}$ & $74 / 74$ & & $\begin{array}{l}61.2 \\
(41.6-92.2)\end{array}$ & \multicolumn{4}{|c|}{$\begin{array}{l}\text { Low due to objective mea- } \\
\text { surement }\end{array}$} \\
\hline Shen 2004 & Syncope & $22 / 51$ & $69.6(55.2)$ & $\begin{array}{l}48.0 \text { (un- } \\
\text { clear) }\end{array}$ & $51 / 52$ & $64.8(90.0)$ & $\begin{array}{l}48.0 \text { (un- } \\
\text { clear) }\end{array}$ & \multicolumn{3}{|c|}{$\begin{array}{l}\text { Low due to objective mea- } \\
\text { surement }\end{array}$} & $\begin{array}{l}\text { Length of stay in hos- } \\
\text { pital only provided } \\
\text { for participants that } \\
\text { were admitted to in- } \\
\text { hospital services. Re- } \\
\text { sults were re-calcu- } \\
\text { lated from days to } \\
\text { hours }\end{array}$ \\
\hline $\begin{array}{l}\text { Strøm } \\
2017 a\end{array}$ & $\begin{array}{l}\text { Internal } \\
\text { medicine } \\
\text { diseases } \\
\text { or condi- } \\
\text { tions }\end{array}$ & $208 / 215$ & & $\begin{array}{l}73 \\
(36-147)\end{array}$ & $210 / 215$ & & $\begin{array}{l}100 \\
(47-169)\end{array}$ & \multicolumn{4}{|c|}{$\begin{array}{l}\text { Low due to objective mea- } \\
\text { surement }\end{array}$} \\
\hline Sun 2014 & Syncope & $62 / 62$ & $29.0(15.0)$ & & $60 / 62$ & $47.0(34.0)$ & & \multicolumn{4}{|c|}{$\begin{array}{l}\text { Low due to objective mea- } \\
\text { surement }\end{array}$} \\
\hline \multicolumn{12}{|c|}{ CI: confidence interval; IQR: interquartile range; $\mathbf{n}$ : number of participants; SD: standard deviation } \\
\hline Table 12. & osts & & & & & & & & & & \\
\hline Study & $\begin{array}{l}\text { Reason } \\
\text { for admis- } \\
\text { sion }\end{array}$ & Definition & & Time point & $\begin{array}{l}\text { Partic- } \\
\text { ipants } \\
\text { evaluat- } \\
\text { ed/ran- } \\
\text { domised } \\
\text { in short- } \\
\text { stay } \\
\text { group (n/ } \\
\text { n) }\end{array}$ & $\begin{array}{l}\text { Ccosts, } \\
\text { mean (SD) }\end{array}$ & $\begin{array}{l}\text { Costs, mec } \\
\text { (IQR) }\end{array}$ & & $\begin{array}{l}\text { Partic- } \\
\text { ipants } \\
\text { evaluat- } \\
\text { ed/ran- } \\
\text { domised } \\
\text { in usu- } \\
\text { al-care } \\
\text { group (n/ } \\
\text { n) }\end{array}$ & $\begin{array}{l}\text { Costs, } \\
\text { mean (SD) }\end{array}$ & $\begin{array}{l}\text { Costs, median } \\
\text { (IQR) }\end{array}$ \\
\hline $\begin{array}{l}\text { Chivite } \\
2008\end{array}$ & $\begin{array}{l}\text { Heart fail- } \\
\text { ure }\end{array}$ & In-hospital costs & & $\begin{array}{l}\text { Index ad- } \\
\text { mission }\end{array}$ & unclear/70 & $\begin{array}{l}\text { EUR } \\
779.43 \\
(573.09)\end{array}$ & & & unclear/69 & $\begin{array}{l}\text { EUR } \\
2311.12 \\
(1847.46)\end{array}$ & \\
\hline
\end{tabular}




\begin{tabular}{|c|c|c|c|c|c|c|c|c|c|}
\hline & & $\begin{array}{l}\text { Total costs (in-hospital costs } \\
\text { and follow-up) }\end{array}$ & $\begin{array}{l}\text { Not clearly } \\
\text { defined }\end{array}$ & & $\begin{array}{l}\text { EUR } \\
2488.60 \\
(956.62)\end{array}$ & & & $\begin{array}{l}\text { EUR } \\
3574.14 \\
(1018,95)\end{array}$ & \\
\hline $\begin{array}{l}\text { Farkouh } \\
1998\end{array}$ & Chest pain & $\begin{array}{l}\text { Costs related to cardiovascu- } \\
\text { lar care }\end{array}$ & 6 months & $\begin{array}{l}\text { un- } \\
\text { clear/212 }\end{array}$ & $\begin{array}{l}\text { not report- } \\
\text { ed }\end{array}$ & not reported & $\begin{array}{l}\text { un- } \\
\text { clear } / 212\end{array}$ & $\begin{array}{l}\text { not report- } \\
\text { ed }\end{array}$ & not reported \\
\hline $\begin{array}{l}\text { Gomez } \\
1996\end{array}$ & Chest pain & Total hospital charges & 30 days & $50 / 50$ & & $\begin{array}{l}\text { USD } 904 \text { (USD } \\
731-\text {-USD 1347) }\end{array}$ & $50 / 50$ & & $\begin{array}{l}\text { USD } 1542 \text { (USD } \\
1142-\text {-US 3845) }\end{array}$ \\
\hline $\begin{array}{l}\text { McDer- } \\
\text { mott } \\
1997 a\end{array}$ & Asthma & Costs during hospitalisation & $\begin{array}{l}\text { Index ad- } \\
\text { mission }\end{array}$ & $\begin{array}{l}\text { un- } \\
\text { clear/110 }\end{array}$ & $\begin{array}{l}\text { USD } 1202 \\
\text { (USD } \\
1343 \text { ) }\end{array}$ & & $\begin{array}{l}\text { un- } \\
\text { clear } / 112\end{array}$ & $\begin{array}{l}\text { USD } 2247 \\
\text { (USD } \\
1110)\end{array}$ & \\
\hline Miller 2010 & Chest pain & Patient charges & $\begin{array}{l}\text { Index ad- } \\
\text { mission }\end{array}$ & $53 / 53$ & & $\begin{array}{l}\text { USD } 2062 \text { (USD } \\
\text { 1918-USD 2367) }\end{array}$ & $57 / 57$ & & $\begin{array}{l}\text { USD } 2680 \text { (USD } \\
\text { 2408-USD 3448) }\end{array}$ \\
\hline & & Cardiac costs & 1 year & $53 / 53$ & & $\begin{array}{l}\text { USD } 2186 \text { (USD } \\
\text { 1957-USD 4308) }\end{array}$ & $57 / 57$ & & $\begin{array}{l}\text { USD } 3850 \text { (USD } \\
\text { 2669-USD 9710) }\end{array}$ \\
\hline $\begin{array}{l}\text { Roberts } \\
1997\end{array}$ & Chest pain & In-hospital costs & $\begin{array}{l}\text { Index ad- } \\
\text { mission }\end{array}$ & $82 / 82$ & $\begin{array}{l}\text { USD } 1528 \\
\text { (USD } \\
1012 \text { ) }\end{array}$ & & $83 / 83$ & $\begin{array}{l}\text { USD } 2095 \\
\text { (USD } \\
2095)\end{array}$ & \\
\hline Ross 2007 & $\begin{array}{l}\text { Transient } \\
\text { ischaemic }\end{array}$ & In-hospital costs & $\begin{array}{l}\text { Index ad- } \\
\text { mission }\end{array}$ & $75 / 75$ & & $\begin{array}{l}\text { USD } 864 \text { (USD } \\
726-U S D \text { 1076) }\end{array}$ & $74 / 74$ & & $\begin{array}{l}\text { USD } 1529 \text { (USD } \\
\text { 1091-USD 2306) }\end{array}$ \\
\hline & & Median 90-day total costs & 90 days & $75 / 75$ & & $\begin{array}{l}\text { USD } 890 \text { (USD } \\
768-U S D \text { 1510) }\end{array}$ & $74 / 74$ & & $\begin{array}{l}\text { USD } 1548 \text { (USD } \\
\text { 1091-USD 2474) }\end{array}$ \\
\hline Sun 2014 & Syncope & In-hospital costs & $\begin{array}{l}\text { Index ad- } \\
\text { mission }\end{array}$ & $62 / 62$ & & $\begin{array}{l}\text { USD } 1190 \text { (USD } \\
870-U S D \text { 1550) }\end{array}$ & $62 / 62$ & & $\begin{array}{l}\text { USD } 1570 \text { (USD } \\
870 ; \text { USD 2370) }\end{array}$ \\
\hline & & $\begin{array}{l}\text { In-hospital costs and fol- } \\
\text { low-up }\end{array}$ & 30 days & $62 / 62$ & & $\begin{array}{l}\text { USD } 1210 \text { (USD } \\
\text { 948-USD 1660) }\end{array}$ & $62 / 62$ & & $\begin{array}{l}\text { USD } 1210 \text { (USD } \\
948-U S D 1660)\end{array}$ \\
\hline
\end{tabular}

CI: confidence interval; EUR: Euro; IQR: interquartile range; $\mathbf{n}$ : number of participants; SD: standard deviation; USD: American dollars

$a_{\text {First }} 130$ of 222 recruited participants were evaluated 


\section{AP PEN DICES}

\section{Appendix 1. Names and synonyms for short-stay units}

1. Accident and Emergency Department Short-stay Unit

2. Acute Admission and Diagnostic Unit

3. Acute Admissions Unit

4. Acute Assessment Unit

5. Acute Care for Elders Unit

6. Acute Diagnostic Unit

7. Acute Elderly Unit

8. Acute Geriatric Unit

9. Acute Geriatrics-based Wards

10.Acute Medical Receiving Unit

11.Acute Medical Unit

12.Acute Medicine Unit

13. Chest Pain Center

14. Chest Pain Observation Unit

15. Clinical Decision Unit

16. Elderly Short-stay Unit

17.Emergency Assessment Unit

18.Emergency Department Asthma and Observation Unit

19. Emergency Department Observation Unit

20.Emergency Diagnostic and Treatment Unit

21.Emergency Medical Assessment/Admissions Unit

22. Emergency Short-stay Unit

23. Extended Evaluation Unit

24.Fast Specialized Ambulatory Care of Medical Disease

25.Fast Track Medical Ward

26.Geriatric Assessment Unit

27.Geriatric Evaluation and Management Unit

28.Immediate Care Clinics

29. Medical Acute Care Unit

30. Medical Assessment and Planning Unit

31. Medical Assessment Unit

32. Medical Short-stay Unit

33. Observation Unit

34.Orthogeriatric Unit for Acute Patients

35. Quick and Early Diagnosis Unit

36.Quick and Early Diagnostic Outpatient Unit

37. Quick diagnostic unit

38. Rapid diagnosis unit

39.Short-stay Clinic

40.Short-stay Hospital Unit

41. Short-stay Medical Unit

42.Short-stay Older Persons Unit

43. Short-stay Unit

44.Short-stay Unit for Older Persons

45.Short-stay Observation Unit

46.Short-stay Respiratory Unit

47. Week Hospital 


\section{Appendix 2. Search strategies}

\section{MEDLINE (Ovid)}

Ovid MEDLINE(R) Epub Ahead of Print, In-Process \& Other Non-Indexed Citations, Ovid MEDLINE(R) Daily, Ovid MEDLINE and Versions(R)

\begin{tabular}{|c|c|c|}
\hline No. & Search terms & Results \\
\hline 1 & $\begin{array}{l}\text { ((emergency or geriatric? or medical) adj3 (planning or evaluation or assess- } \\
\text { ment or asthma or observation or diagnos* or admission? or treatment) adj3 } \\
\text { (unit? or ward?)).ti,ab. }\end{array}$ & 1811 \\
\hline 2 & ((quick or early or rapid) adj3 diagnos* adj3 (unit? or ward?)).ti,ab. & 96 \\
\hline 3 & week hospital?.ti,ab. & 112 \\
\hline 4 & short stay ${ }^{\star}$.ti. & 583 \\
\hline 5 & (acute adj3 hospital adj3 (unit? or ward?)).ti,ab. & 544 \\
\hline 6 & (chest pain adj3 (center? or centre? or unit? or ward?)).ti,ab. & 674 \\
\hline 7 & (clinical decision adj (unit? or ward?)).ti,ab. & 41 \\
\hline 8 & (extended evaluation adj (unit? or ward?)).ti,ab. & 1 \\
\hline 9 & 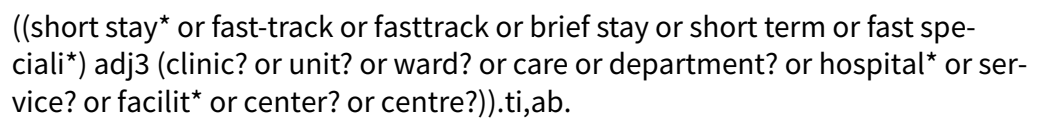 & 4668 \\
\hline 10 & (immediate care adj (clinic? or unit? or ward? or centre? or center?)).ti,ab. & 7 \\
\hline 11 & (observation adj (unit? or ward?)).ti,ab. & 874 \\
\hline 12 & $\begin{array}{l}\text { (acute adj3 (admission? or diagnos* or assessment or care or elderly or geri- } \\
\text { atric? or orthogeriatric? or medical or medicine) adj3 (unit? or ward?)).ti,ab. }\end{array}$ & 3666 \\
\hline 13 & (orthogeriatric adj (unit? or ward?)).ti,ab. & 64 \\
\hline 14 & or/1-13 & 11811 \\
\hline 15 & exp randomized controlled trial/ & 515248 \\
\hline 16 & controlled clinical trial.pt. & 101721 \\
\hline 17 & randomi\#ed.ti,ab. & 582212 \\
\hline 18 & placebo.ab. & 210465 \\
\hline 19 & randomly.ti,ab. & 312358 \\
\hline 20 & Clinical Trials as topic.sh. & 202436 \\
\hline 21 & trial.ti. & 203181 \\
\hline 22 & or/15-21 & 1300464 \\
\hline
\end{tabular}




\begin{tabular}{lll} 
exp animals/ not humans/ & 4810234 \\
\hline 24 & 22 not 23 & 1201092 \\
\hline 25 & 14 and 24 & 1126 \\
\hline
\end{tabular}

\section{Embase (Ovid)}

Embase $<1974$ to 2017 December 12>

\begin{tabular}{|c|c|c|}
\hline No. & Search terms & Results \\
\hline 1 & $\begin{array}{l}\text { ((emergency or geriatric? or medical) adj3 (planning or evaluation or assess- } \\
\text { ment or asthma or observation or diagnos* or admission? or treatment) adj3 } \\
\text { (unit? or ward?)).ti,ab. }\end{array}$ & 2644 \\
\hline 2 & ((quick or early or rapid) adj3 diagnos ${ }^{\star}$ adj3 (unit? or ward?)).ti,ab. & 134 \\
\hline 3 & week hospital?.ti,ab. & 176 \\
\hline 4 & short stay ${ }^{\star}$.ti. & 711 \\
\hline 5 & (acute adj3 hospital adj3 (unit? or ward?)).ti,ab. & 720 \\
\hline 6 & (chest pain adj3 (center? or centre? or unit? or ward?)).ti,ab. & 988 \\
\hline 7 & (clinical decision adj (unit? or ward?)).ti,ab. & 82 \\
\hline 8 & (extended evaluation adj (unit? or ward?)).ti,ab. & 1 \\
\hline 9 & 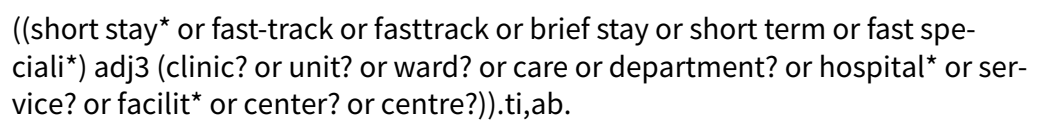 & 6091 \\
\hline 10 & (immediate care adj (clinic? or unit? or ward? or centre? or center?)).ti,ab. & 10 \\
\hline 11 & (observation adj (unit? or ward?)).ti,ab. & 1254 \\
\hline 12 & $\begin{array}{l}\text { (acute adj3 (admission? or diagnos* or assessment or care or elderly or geri- } \\
\text { atric? or orthogeriatric? or medical or medicine) adj3 (unit? or ward?)).ti,ab. }\end{array}$ & 5140 \\
\hline 13 & (orthogeriatric adj (unit? or ward?)).ti,ab. & 140 \\
\hline 14 & or/1-13 & 16231 \\
\hline 15 & random*.ti,ab. & 1271058 \\
\hline 16 & factorial* ${ }^{*}$ ti,ab. & 31916 \\
\hline 17 & (crossover ${ }^{\star}$ or cross over ${ }^{\star}$ ).ti,ab. & 92321 \\
\hline 18 & $\left(\left(\right.\right.$ doubl$^{\star}$ or singl $\left.{ }^{\star}\right)$ adj blind $\left.{ }^{\star}\right) \cdot$.ti,ab. & 204646 \\
\hline
\end{tabular}


(Continued)

\begin{tabular}{lll}
\hline 19 & (assign* or allocat* or volunteer* or placebo*).ti,ab. & 886972 \\
\hline 20 & crossover procedure/ & 54460 \\
\hline 21 & single blind procedure/ & 30503 \\
\hline 22 & randomized controlled trial/ & 486392 \\
\hline 23 & double blind procedure/ & 146288 \\
\hline 24 & or/15-23 & 1966881 \\
\hline 25 & exp animal/ not human/ & 4934761 \\
\hline 26 & 24 not 25 & 1762445 \\
\hline 27 & 14 and 26 & 1915
\end{tabular}

\section{The Cochrane Library (Wiley)}

\begin{tabular}{|c|c|c|}
\hline No. & Search terms & Results \\
\hline$\# 1$ & $\begin{array}{l}\text { ((emergency or geriatric }{ }^{\star} \text { or medical) near/3 (planning or evaluation or assess- } \\
\text { ment or asthma or observation or diagnos }{ }^{\star} \text { or admission* or treatment) near/3 } \\
\text { (unit* or ward*)):ti,ab }\end{array}$ & 149 \\
\hline$\# 2$ & ((quick or early or rapid) near/3 diagnos* near/3 (unit* or ward $\left.\left.{ }^{\star}\right)\right): \mathrm{ti}, \mathrm{ab}$ & 7 \\
\hline \#3 & week next hospital*:ti,ab & 38 \\
\hline \#4 & short next stay*:ti & 56 \\
\hline \#5 & (acute near/3 hospital near/3 (unit* or ward*)):ti,ab & 74 \\
\hline \#6 & 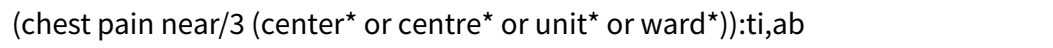 & 55 \\
\hline \#7 & (clinical decision near/1 (unit* or ward $\left.{ }^{\star}\right)$ ):ti,ab & 2 \\
\hline$\# 8$ & (extended evaluation near/1 (unit* or ward $\left.{ }^{\star}\right)$ ):ti,ab & 1 \\
\hline \#9 & $\begin{array}{l}\text { ((short stay }{ }^{\star} \text { or fast-track or fasttrack or brief stay or short term or fast spe- } \\
{\text { ciali*) near } / 3\left(\text { clinic }^{\star} \text { or unit }\right.}^{\star} \text { or ward } \text { or care or department }^{\star} \text { or hospital }{ }^{\star} \text { or } \\
\left.\left.\text { service }^{\star} \text { or facilit* } \text { or center }^{\star} \text { or centre }{ }^{\star}\right)\right): t i, a b\end{array}$ & 1248 \\
\hline$\# 10$ & (immediate care near $/ 1$ (clinic ${ }^{\star}$ or unit ${ }^{\star}$ or ward ${ }^{\star}$ or centre ${ }^{\star}$ or center $)$ ):ti,ab & 416 \\
\hline \#11 & (observation near/1 (unit* or ward*)):ti,ab & 97 \\
\hline$\# 12$ & $\begin{array}{l}\text { (acute near/3 (admission* or diagnos* or assessment or care or elderly or geri- } \\
\left.\text { atric }^{\star} \text { or orthogeriatric* or medical or medicine) near } 3\left(\text { unit }^{\star} \text { or ward }^{\star}\right)\right): \text { ti,ab }\end{array}$ & 363 \\
\hline \#13 & (orthogeriatric near/1 (unit* or ward*)):ti,ab & 8 \\
\hline
\end{tabular}




\section{ClinicalTrials.gov}

\section{Search terms}

[Titles]

acute admission OR acute assessment OR acute care OR acute diagnostic OR acute elderly OR acute geriatric OR acute medical OR acute medicine OR chest pain center OR clinical decision OR emergency assessment

[Titles]

emergency diagnostic and treatment OR emergency medical assessment OR emergency medical admission OR extended evaluation OR fast specialized OR fast track OR immediate care OR medical assessment

[Titles] observation unit OR quick and early OR quick diagnostic OR rapid diagnosis OR short-stay OR week hospital OR AMU OR SSU

\section{WHO International Clinical Trials Registry Platform (ICTRP)}

"acute admission and diagnostic unit" OR "acute admissions unit" OR "acute assessment unit" OR "acute care for elders unit" OR "acute diagnostic unit" OR "acute elderly unit" OR "acute geriatric unit" OR "acute geriatrics-based wards" OR "acute medical receiving unit" OR "acute medical unit" OR "acute medicine unit" OR "chest pain center" OR "clinical decision unit" OR "emergency assessment unit" OR "emergency diagnostic and treatment unit" OR "emergency medical assessment unit" OR "emergency medical admissions unit" OR "extended evaluation unit" OR "fast specialized ambulatory care of medical disease" OR "fast track medical ward" OR "geriatric assessment unit" OR "geriatric evaluation and management unit" OR "immediate care clinics" OR "medical acute care unit" OR "medical assessment and planning unit" OR "medical assessment unit" OR "observation unit" OR "orthogeriatric unit" OR "quick and early diagnosis unit" OR "quick and early diagnostic outpatient unit" OR "quick diagnostic unit" OR "rapid diagnosis unit" OR "short-stay" OR "week hospital"

\section{Appendix 3. Screening algorithm for titles and abstracts}

1. Does the trial compare treatment in a short-stay unit with usual care?

2. Does the trial include adult participants ( $\geq 18$ years)

3. Does the trial include patients admitted to treatment for an internal medical disease or condition (participants)?

4. Does the trial meet design criteria; either 1) randomised trial, 2) randomised step wedge design trial, 3) cluster randomised trial?

If the answer to all the questions is "yes" or "not clear", the entire text of the paper will be examined. 


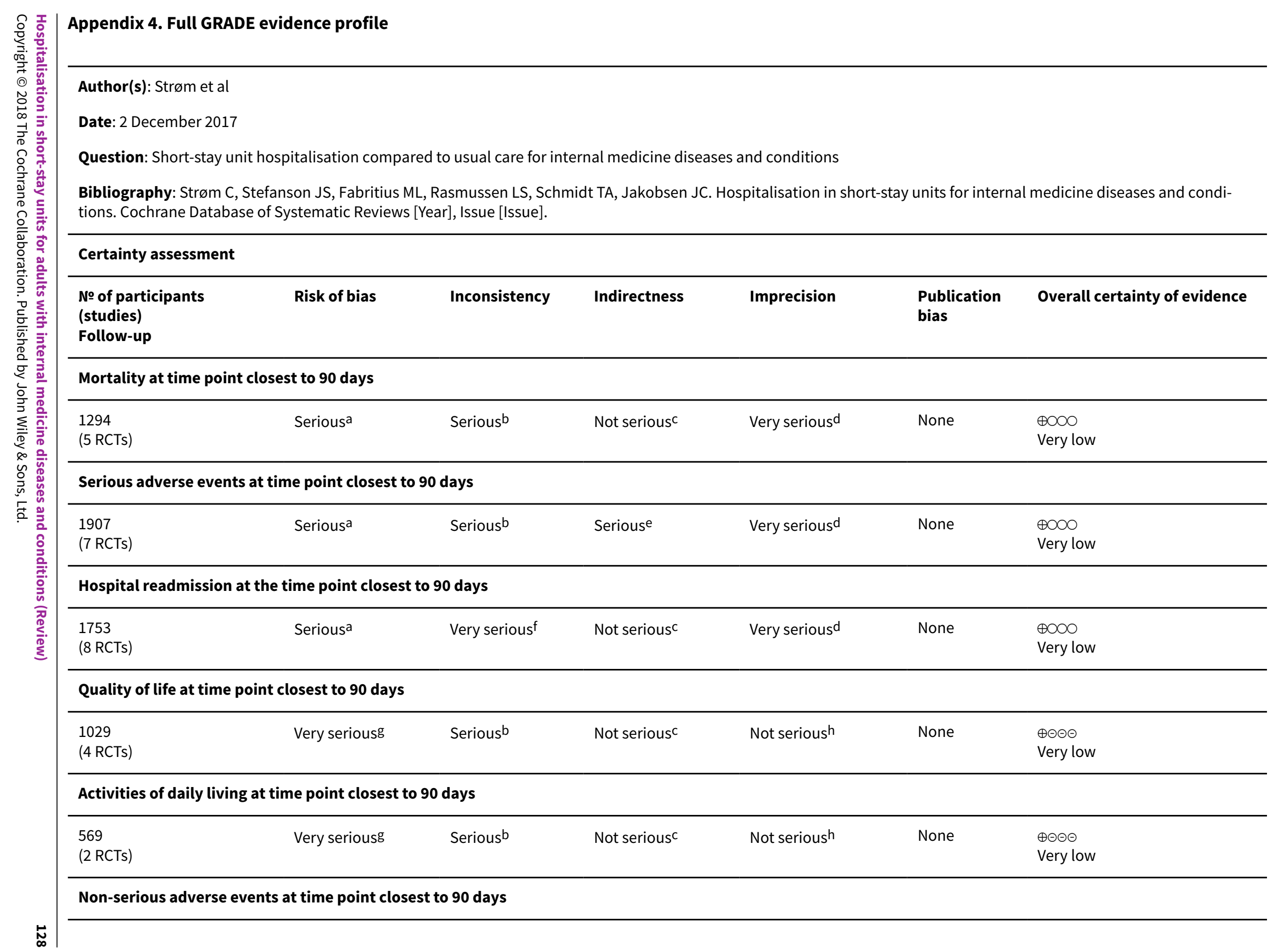

Bibliography: Strøm C, Stefanson JS, Fabritius ML, Rasmussen LS, Schmidt TA, Jakobsen JC. Hospitalisation in short-stay units for internal medicine diseases and condiCochrane Database of Systematic Reviews [Year], Issue [Issue]. 


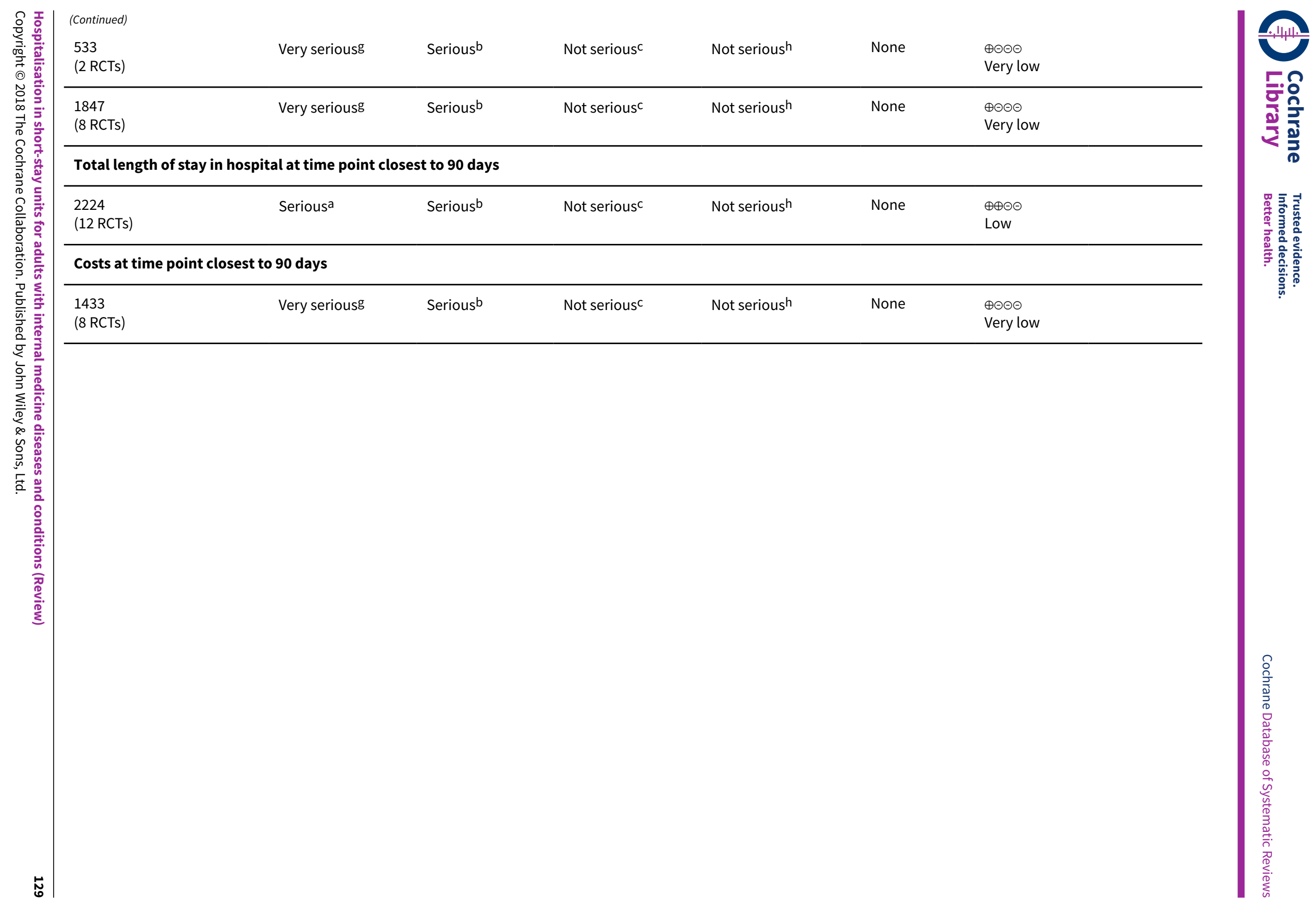


Cl: Confidence interval; RR: Risk ratio

aDowngraded one level for serious risk of bias due to all trials being at high risk of bias, but because the outcome is a more objective outcome, lack of blinding of participants, personnel and outcome assessors may not bias the outcome as much.

bDowngraded one level for clinical heterogeneity among the included trials.

cNot downgraded for indirectness.

dDowngraded two levels due to low number of events and the $95 \% \mathrm{Cl}$ around the pooled estimate of effect included both appreciable benefit or appreciable harm.

eDowngraded one level due to indirect evidence (surrogate outcome measures for adverse events).

fDowngraded two levels for clinical and statistical heterogeneity among the included trials

gDowngraded two levels for very serious risk of bias due to all trials being at high risk of bias.

hNot downgraded for imprecision. We were not able to evaluate estimate of effect in meaningful meta-analysis.

\section{CONTRIBUTIONS OF AUTHORS}

Designing the protocol: CS, MF, LS, TAS, JCJ

Conceiving the review: CS, JS, MF, LS, TAS, JCJ

Retrieval of studies: CS, PM

Selection of studies for inclusion/exclusion and data collection: CS, JS, MF, JCJ

Analyses, writing of review: CS

Critical feedback on analyses and drafts of review: JS, MF, LS, TAS, JCJ

\section{DECLARATIONS OF INTEREST}

Three review authors of this review (CS, LS, TS) conducted one of the included trials (Strøm 2017a). In this review, two review authors who had not been involved in the trial (MF and JS) assessed it.

Camilla Strøm has no other conflicts of interest.

Jakob Skulason Stefanson has no conflicts of interest.

Maria Louise Fabritius has no conflicts of interest.

Lars S Rasmussen has no other conflicts of interest.

Thomas A Schmidt has no other conflicts of interest.

Janus C Jakobsen has no conflicts of interest.

\section{SOURCES OF SUPPORT}

\section{Internal sources}

- Faculty of Health and Medical Sciences, University of Copenhagen, Denmark.

CS has received a PhD stipend, this review is part of the PhD project.

- Region Zealand, Denmark.

CS has received a PhD stipend, this review is part of the PhD project.

\section{External sources}

- Tryg Foundation, Denmark.

LS has received funding from Tryg Foundation for other projects.

- Region Zealand Research Foundation, Denmark.

CS has received a EUR 10,000 grant for a PhD project, this review will be part of the project. 


\section{DIFFERENCES BETWEEN PROTOCOLAND REVIEW}

Jakob Skulason Stefanson was added as a review author (second author); he was involved in the study selection process, data collection process of all included trials, and provided feedback on the manuscript drafts.

We were not able to conduct the following subgroup analyses on the outcomes mortality, serious adverse events, and hospital readmission:

- comparison of the effect of short-stay unit hospitalisation compared with usual care between trials with low and lower risks of bias compared with trials with trials with high risk of bias, because all trials were judged to be of high risk of bias;

- comparison of the effect of short-stay unit hospitalisation compared with usual care between trials investigating emergency department-based short-stay units compared with non-emergency department based short-stay units, because all trials investigated emergency department-based short-stay units;

- comparison of the effect of short-stay unit hospitalisation compared with usual care between trials investigating protocol-specific interventions in short-stay unit compared with non-protocol-specific interventions in short-stay unit;

- comparison of the effect of short-stay unit hospitalisation compared with usual care between trials investigating units with a targeted length of stay in hospital of five or fewer days compared with no defined or longer length of stay in hospital for participants, because all trials investigated short-stay units with a targeted length of stay in hospital shorter than five days.

We were not able to conduct the following sensitivity analyses:

- restricting the analyses to trials with a low risk of bias, as specified in Assessment of risk of bias in included studies;

- restricting the analyses to trials that evaluated outcomes at least once within six months of inclusion.

We were not able to conduct any sensitivity analyses for continuous outcomes.

We were not able to conduct meta-analyses for the outcomes quality of life, activities of daily living, non-serious adverse events, transfer to other department, and length of stay in hospital, because data were too sparse or reported in an unusable way.

It was impossible to evaluate treatment effects according to the predefined values for clinical significance for each outcome, because data were either lacking or sparse with very wide confidence intervals that showed serious imprecision (imprecise confidence estimates).

We did not use the eight-step procedure to assess whether or not the thresholds for statistical and clinical significance were crossed, as stated in the protocol. This change was made to comply with EPOC guidance at the request of the EPOC editors.

\section{IN DEX TERMS}

\section{Medical Subject Headings (MeSH)}

*Internal Medicine; *Length of Stay; Activities of Daily Living; Asthma; Atrial Fibrillation; Chest Pain; Heart Failure; Hospital Mortality; Hospital Units [ ${ }^{\star}$ classification]; Ischemic Attack, Transient; Patient Readmission; Quality of Life; Randomized Controlled Trials as Topic; Syncope; Time Factors

\section{MeSH check words}

Adult; Humans 